\title{
SUBDOSA - A Computer \\ Program for Calculating \\ External Doses from \\ Accidental Atmospheric Releases of Radionuclides
}

June 1975

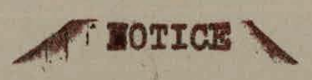

PORTIONS OF THIS REPORT ARE TLIEGIBLE. IE has been reproduced from the best availablo copy to permit the broadest possible availey ability.

Prepared for the U.S. Energy

Research and Development Administration under Contract AT(45-1): 1830 


\section{DISCLAIMER}

This report was prepared as an account of work sponsored by an agency of the United States Government. Neither the United States Government nor any agency Thereof, nor any of their employees, makes any warranty, express or implied, or assumes any legal liability or responsibility for the accuracy, completeness, or usefulness of any information, apparatus, product, or process disclosed, or represents that its use would not infringe privately owned rights. Reference herein to any specific commercial product, process, or service by trade name, trademark, manufacturer, or otherwise does not necessarily constitute or imply its endorsement, recommendation, or favoring by the United States Government or any agency thereof. The views and opinions of authors expressed herein do not necessarily state or reflect those of the United States Government or any agency thereof. 


\section{DISCLAIMER}

Portions of this document may be illegible in electronic image products. Images are produced from the best available original document. 


\title{
NOTICE
}

This report was prepared as an account of work sponsored by the United States Government. Neither the United States nor the United States Energy Research and Development Administration, nor any of their employees, nor any of their contractors, subcontractors, or their employees, makes any warranty, express or implied, or assumes any legal liability or responsibility for the accuracy, completeness or usefulness of any information, apparatus, pruducl un process diselosed, or represents that its use would not infringe privately owned rights.

\section{PACIFIC NORTHWEST LABORATORY}

operated by

BATTELLE

for the

\section{U.S. ENERGY RESEARCH AND DEVELOPMENT ADMINISTRATION}

Under Contract E(45-1)-1830

\author{
Primeed in the United States of America \\ Available from \\ National Technical Information Service \\ U.S. Department of Commerce \\ $52 a 5$ Port Royal Road \\ Springfield, Virginia 22151
}

Price: Printed Copy \$7.60; Microfiche $\$ 2.25$ 


\section{SUBDOSA - A COMPUTER PROGRAM FOR CALCULATING \\ EXTERNAL DOSES FROM ACCIDENTAL ATMOSPHERIC \\ RELEASES OF RADIONUCLIDES}

by D. L. Strenge, E. C. Watson and J. R. Houston Occupational and Environmental Safety Department

June 1975

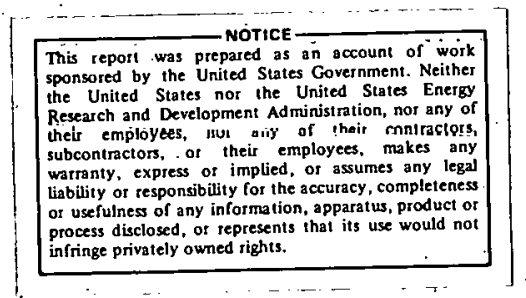

Battelle,

Pacific Northwest Laboratories

Richland, Washington 99352 


\section{CONTENTS}

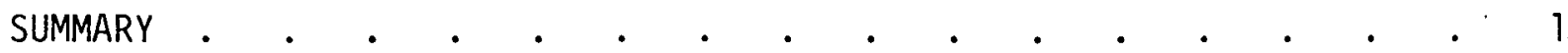

INTRODUCTION •

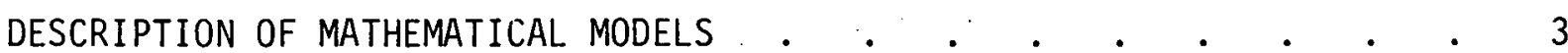

Total Body Tissue Dose from Gamma Radiation . . . . . . . 3

Surface Tissue Dose from Beta Radiation . . . . . . . . . . . 99

Tissue Depth Dose from Beta Radiation . . . . . . . . . 10

Skin, Eye, Male Gonad, and Total Body Dose . . . . . . 13

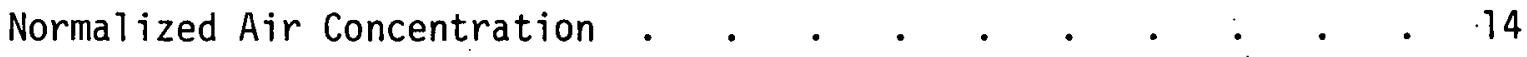

COMPUTER PROGRAM .

BELI • • • • • • •

BIVAR . • . . . . . . . . . . . . . . . 18

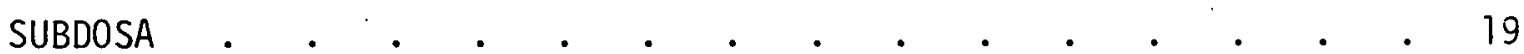

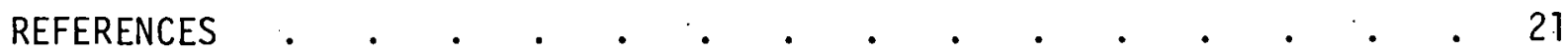

APPENDIX A: DATA LIBRARIES .

APPENDIX B: PROGRAM LISTING • • •

APPENDIX C: INPUT PREPARATION

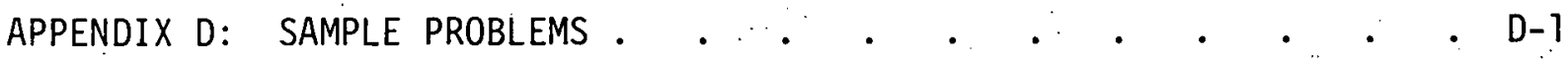

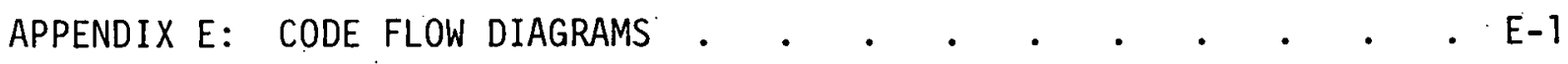


SUBDOSA - A COMPUTER PROGRAM FOR CALCULATING EXTERNAL DOSES

FROM ACCIDENTAL ATMOSPHERIC RELEASES OF RADIONUCLIDES

D. L. Strenge, E. C. Watson and J. R. Houston

\section{SUMMARY}

A computer program, SUBDOSA, has been developed for calculating external gamma and beta doses to individuals from the accidental release of radionuclides to the atmosphere. Characteristics of SUBDOSA are:

- Doses from both gamma and beta radiation are calculated as a function of depth in tissue, summed and reported as skin, eye, gonadal and total body dose.

- Doses are calculated for releases within each of several release time intervals. Up to six time intervals can be allowed and separate nuclide inventories and atmospheric dispersion conditions are considered for each time interval.

- Radioactive decay is considered during the release and/or transit using a chain decay scheme with branching to account for transitions to and from isomeric states.

- The dose from gamma radiation is calculated using a numerical - integration technique to account for the finite size of the plume.

- The program computes and lists the normalized air concentrations at ground level as a function of distance from the point of release. 


\section{INTRODUCTION}

The computer program SUBDOSA has" been developed for the purpose of calculating the dose to humans following accidental atmospheric releases of radionuclides. SUBDOSA calculates the dose from airborne radionuclides external to the body and does not consider the dose resulting from radionuclides deposited in the body and its organs via inhalation. Doses are calculated as a function of: quantity released, duration of release, atmospheric conditions during the release, and horizontal distance from the release point.

The dose to organs of reference in adults from airborne radionuclides external to the human body is primarily dependent. upon the type of radiation, the energy of the radiation and the spatial distribution of the airborne radionuclides surrounding the human receptor. The energy and type of radiation are characteristic of each radionuclide. Spatial distribution of airborne radionuclides is determined by the quantity released, the duration of release, the atmospheric conditions prevailing during the release and the distance between the dose receptor and the point of release.

The organs of reference most frequently considered in external dose calculations are the skin, lens of the eye and total body. Corresponding tissue depths for these organs used in SUBDOSA are $0.007,0.1$ and $5 \mathrm{~cm}$ respectively. The male gonads are less often considered in dose calculations but when such calculations are made with SUBDOSA, the tissue depth is $1 \mathrm{~cm}$. 


\section{DESCRIPTION OF MATHEMATICAL MODELS}

Mathematical models used to calculate gamma and beta doses and' normalized air concentrations are described in this section. The expressions of gamma dose are discussed first followed by the beta dose and normalized air concentration equations...

\section{TOTAL BODY TISSUE DOSE FROM GAMMA RADIATION}

The basic equation for the external total body tissue dose rate from a gamma emitting radionuclide present in an incremental volume of a cloud is:

$$
d_{\gamma}=k_{k} \frac{E_{\gamma} x B\left(\mu_{k} r\right) \exp \left(-\mu_{k} r\right)}{4 \pi r^{2}} d x d y d z
$$

where:

$d_{\gamma}$ - incremental dose rate to tissue, from gamma radiation emitted from an incremental cloud volume, $d x, d y, d z$ at a distance $r$ meters from the point of interest, $\mathrm{rad} / \mathrm{sec}$

$x$ - concentration in incremental cloud volume, $\mathrm{C} i / \mathrm{m}^{3}$

$E_{\gamma} \cdot$ photon energy, MeV/disintegration

$B\left(u_{k} r\right) \cdot$ dose buildup factor for air.

$\mu_{k}$ - total linear attenuation coefficient in air, $\mathrm{m}^{-1}$, for photons with gamma energy. in energy group $k$

$K_{k}$ - dose conversion factor, $\left(\mathrm{rad} \cdot \mathrm{m}^{2}\right) /(\mathrm{Ci} \cdot \mathrm{sec})$ per $\mathrm{MeV}$ disintegration.

The constant $k_{k}$ is:

$$
\begin{aligned}
K_{k} & =\frac{3.70 \times 10^{10} \frac{\mathrm{dis}}{\mathrm{Ci} \cdot \mathrm{sec}} 1.60 \times 10^{-6} \frac{\mathrm{erg}}{\mathrm{MeV}} 10^{-4} \frac{\mathrm{m}^{2}}{\mathrm{~cm}^{2}}}{100 \frac{\mathrm{ergs}}{\mathrm{g} \cdot \mathrm{rad}}}\left(\frac{\mu_{\mathrm{d}}}{\rho}\right)_{k} \\
& =0.0592\left(\frac{\mu_{\mathrm{a}}}{\rho}\right)_{k}
\end{aligned}
$$


where:

$$
\left(\frac{\mu_{a}}{\rho}\right)_{k} \begin{aligned}
& \text { - mass absorption coefficient, } \mathrm{cm}^{2} / g \text { in tissue for } \\
& \text { average gamma energy, of energy group } k . . .
\end{aligned}
$$

A quadratic expression is used to calculate the dose buildup factor.

$$
B\left(\mu_{k} r\right)=1+A_{k} \mu_{k} r+\alpha_{k}\left(\mu_{k} r\right)^{2}
$$

where:

$A_{k}$ and $\alpha_{k}$ are empirical constants determined to fit buildup factor data of Berger.

Since dose rate is a function of $\gamma$-ray energy, the gamma spectrum has heen divided into energy groups. The increminlal duse 15 calculated separately as a function of photon energy and integrated over the cloud volume to obtain dose rate factors for each energy group. The dose rate factors are coupled with radionuclide release to give individual.dose rates. The total dose is calculated as the time integral of dose rate.

Before integrating Equation (1), it is first necessary to describe mathematically the plume concentration, $x$. To obtain a useful expression, several assumptions are necessary:

- Diffusion along the direction of cloud travel can be ignored.

- Vertical ard lateral crosswind concentration is normally distributed and the standard deviations are a function of atmospheric stability and distance from the release point. In some dispersion models, the standard deviations are wind speed dependent.

- The dose receptor is at ground level.

- Cloud depletion by fallout, washout and rainuut can be described by a factor dependent on the distance of travel and is independent of travel time and displacement from the centerline.

- The concentration of radionuclides in the cloud at distances beyond three standard deviations in the vertical and lateral directions is insignificant and therefore makes little 
contribution to the dose rate. Radionuclide concentrations at distances greater than $\pm 800 \mathrm{~m}$ in the direction of cloud travel can also be ignored.

- Radionuclide decay is calculated for each radionuclide based on travel time to the exposure point. This ... concentration is used for all downwind integration. points (i.e., over $\pm 800 \mathrm{~m}$ from the exposure point).

The plume concentration at a vertical position $z$ and lateral position $y$ is given by: (2)

$$
x=Q^{\prime} \cdot \frac{\exp \left[-(z-h)^{2} / 2 \sigma_{z}^{2}-y^{2} / 2 \sigma_{y}^{2}\right]}{2 \pi \bar{u}_{h} \sigma_{y} \sigma_{z}}
$$

where:

$\bar{u}_{h}$ - average wind speed in direction of travel measured at height of release, $\mathrm{m} / \mathrm{sec}$

$\sigma_{z}$ - crosswind vertical standard deviation of cloud concentration, $m$

$\sigma_{y} \cdot$ crosswind lateral standard deviation of cloud concentration, $\mathrm{m}$

$h$ - height of release, $m$

.y lateral displacement from cloud centerline of incremental element of cloud, $m$

$z$ - height of incremental element of cloud, $m$

Q '. rate of release from source, corrected for decay during transit: to exposure point, curies/sec:

Equation (3) does not include a factor of two for ground reflection." Determination of $\sigma_{y}$ and $\sigma_{z}$ is discussed in a subsequent section. 
The dose rate from all radionuclides within the plume is obtained by performing a space integration of Equation (1) with $x$ defined by Equation (3). For a given nuclide and distance from release point the dose rate is (rads/sec):

$$
D_{\gamma}^{\prime}=Q^{\prime} \sum_{k=1}^{\substack{\text { Energy } \\
\text { Groups }}} \begin{gathered}
\text { Photons in } \\
\text { Group } k
\end{gathered} \sum_{\ell=1}\left(A_{k} E_{\gamma k}\right)_{\ell}
$$

where:

$$
\begin{aligned}
& D_{\gamma}^{\prime} \cdot \text { Dose rate, } \mathrm{rad} / \mathrm{sec} \\
& A_{k \ell} \cdot \text { abundance of } \ell \text {-th photon in energy group } k \text {, } \\
& \text { photons/disintegration } \\
& E_{\gamma k \ell} \cdot \text { energy of } \ell \text {-th photon in energy group } k \text {, } \\
& \mathrm{MeV} / \text { photon } \\
& \mathrm{DRF}_{k}=\frac{1}{2 \pi \bar{u}_{h}} \int_{x_{1}}^{x_{2}} \int_{z_{1}}^{z_{2}} \frac{\exp -\left[(z-h)^{2} / 2 \dot{\sigma}_{z}^{2}\right]}{\sigma_{z}} . \\
& \int_{y_{1}}^{y_{2}} \frac{B\left(\mu_{k} r\right) \exp \left(-y^{2} / 2 \sigma y^{2}\right) \exp \left(-\mu_{k} r\right) k_{k}}{4 \pi r^{2} \sigma_{y}} d x d y d z
\end{aligned}
$$

The Dose Rate Factors, DRF, have units of $\mathrm{rad} / \mathrm{sec}$ per curie (at the distance of interest) per MeV/disintegration from photons in energy group $k$. Equation (5) is integrated using a numerical technique as described in Reference 3. The dose rate from all nuclides is obtained by adding the contributions from all nuclides present. The total dose is the time integral of the total dose rate. Since the release rate is the only time dependent parameter, the total dose may be expressed as:

$$
D_{\gamma}=\int_{0}^{T}{ }^{T} D_{\gamma}^{\prime} d t
$$


where:

$$
T_{r} \cdot \text { duration of release period. }
$$

Often the total release is given as input and no time integration is necessary. However, if the total release is not known, it may be calculated from knowledge of the initial amount present and the nuclide decay schemes. The nuclide decay scheme used by SUBDOSA accounts for chain decay including transitions to and from isomeric states. The general equation for such a scheme is:

$$
\frac{d M_{i}}{d t}=\sum_{j} \lambda_{j} k_{j}^{i} M_{j}-\lambda_{j} M_{i}
$$

where:

$M_{i}$ - rate of release for nuclide $i$ after decay for travel time $T$, atoms/sec

$M_{j} \bullet$ rate of release of parent nuclide $j$, atoms $/ \mathrm{sec}$

$\lambda_{j}$ - decay rate constant for nuclide $i\left(\mathrm{sec}^{-1}\right)$

$K_{j}^{i} \cdot$ branching ratio for nuclide $j$ decaying to nuclide $i$.

Equation (7) may be solved analytically to obtain the rate of release at any time $t$ as:

$$
M_{j}(t)=\sum_{k=1}^{n} A_{k} e^{-\lambda k^{t}}
$$

where:

$n$ - position in decay chain of nuclide $i$

$\lambda_{k}$ - physical decay constant for chain member $k$

$A_{k}$ - coefficient composed of decay fractions and physical decay constants for nuclides in the chain

$k$ - the summation is over all nuclides of the chain down to nuclide $i$. 
When the nuclide inventory is given as the amount present at the start of the release period, it is necessary to integrate over the release period to determine the actual release for Equation (6). If release is assumed to be constant (except for decay) $M_{j}$ of Equation (7) can be replaced by:

$$
M_{i}(t)=\frac{Q_{j}(t)}{T r}
$$

where:

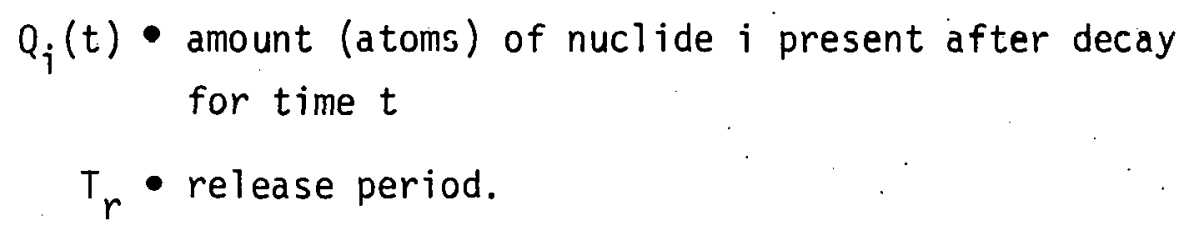

The resulting equation can be solved in the same manner as Equation. (7) to obtain a solution corresponding to Equation (8):

$$
Q_{j}^{\prime}(t)=\frac{Q_{j}(t)}{T_{r}}=\sum_{k=1}^{n} \frac{A_{k}}{T_{r}} e^{-\lambda{ }_{k} t}
$$

where the terms are as previousily defined, except that $Q_{j}{ }^{\prime}(t)$ is in units of atoms. This equation is easily integrated over the release period to give the total release for use in Equation (6) as:

$$
Q_{j}\left(T_{r}\right)=\int_{0}^{T_{r}} Q_{j}{ }^{\prime}(t) d t=\sum_{k=1}^{n} \frac{\Lambda_{k}}{T^{\lambda_{k}}}\left(1-e^{-\lambda_{k} T_{r}}\right)
$$

The total release $Q_{j}\left(T_{r}\right)$ must be converted from atoms to curies for use in Equation (6). In practice the decay calculation, Equation (8), and time integration, Equation (11), are performed in units of curie.sec, i.e., curies divided by the physical decay constant for the nuclide. Conversinn to units of curies/sec or curies is then made by simply multiplying the results by the physical decay constant.

Equation (6) gives the gamma dose to the surface tissue. The dose to tissue below the surface is calculated by applying an attenuation factor to the dose rate factor of Equation (4). 


$$
D_{\gamma}^{\prime}(d)=Q^{\prime} \sum_{k=1}^{\text {Group }}\left(1+\mu_{k} d\right) D R F_{k} e^{-\mu_{k}^{\prime d}} \sum_{l=1}^{\text {Photons in }}{ }^{\text {Group } k}\left(A_{k} E_{\gamma k}\right)_{l}
$$

where the terms are as defined for Equation (4) and:

$$
\begin{gathered}
D_{\gamma}^{\prime}(d) \text { - gamma tissue dose rate at a depth } d \text {, rads/sec } \\
d \text { - depth in tissue of exposure point, cm } \\
\mu_{k}^{\prime} \text { - linear total attenuation coefficient of water } \\
\ldots \text { for photons in energy group } k, \mathrm{~cm}^{-1} \text {. }
\end{gathered}
$$

The results of Equation (12), expressed as $D_{\gamma}^{\prime}(d) / Q^{\prime}$, are used in Equation (6) to determine the gamma tissue dose at depth $\stackrel{\gamma}{d}$.

SURFACE TISSUE DOSE FROM BETA RADIATION

The beta dose calculation is considerably simpler than the gamma dose calculation. Because of the short range of beta particles in air, the radionuclide cloud may be assumed to be semi-infinite in dimension. With this assumption the beta dose to surface tissue is: ${ }^{(2)}$

$$
D_{B}=0.229 \dot{\bar{E}}_{B} \psi
$$

where:

$\psi$ - time integrated air concentration at the receptor, $\mathrm{Ci} \cdot \mathrm{sec} / \mathrm{m}^{3}$

$\bar{E}_{\beta}$ - effective beta energy for the nuclide being considered, MeV/disintegration

$$
0.229=\frac{\left(1.6 \times 10^{-6} \frac{\mathrm{ergs}}{\mathrm{MeV}}\right)\left(3.7 \times 10^{10} \frac{\mathrm{dis}}{\mathrm{sec} \cdot \text { curie }}\right)}{\left(1293 \mathrm{~g} / \mathrm{m}^{3}\right)\left(100 \frac{\mathrm{ergs}}{\mathrm{gamma} \text { rad }}\right)}(1 / 2)
$$

The factor of $1 / 2$ is to account for body self shielding from half the radioactive cloud. . The total beta dose is the sum of dose contributions from each nuclide. 
The time integral of air concentration is calculated from the instantaneous air concentration of Equation (3) as:

$$
\psi=2\left(\frac{x}{Q^{\prime}}\right) \int_{0}^{T_{r}} Q^{\prime} d t
$$

The integration is performed as for the gamma dose calculation. Since the exposure point is assumed to be on or below the cloud centerline at ground leve1, the term $\exp \left(-y^{2} / 2 \sigma_{y}\right)$ is not included in evaluating $x / Q^{\prime}$ for the beta dose calculation. The factor of 2 is for plume reflection about the ground plane.

\section{TISSUE DEPTH DOSE FROM BETA RADIATION}

Equation (13) determines the beta dose to surface tissue. To determine beta dose to tissue below the surface, the effective beta energy at the given tissue depth must be known.

To correctly determine the effective beta energy emitted by a particular nuclide, the contributions from a 17. beta peaks of the spectrum must be considered. A computer program has been developed to calculate the effective beta energy at a tissue depth of $\mathrm{d} \mathrm{mg} / \mathrm{cm}^{2}$ using beta spectral data as input. In Appendix A the spectral data used are described together with the resulting effective beta energies as a furction of tissue depth.

The effective beta energy for a given emission peak is calculated as the integral of the beta spectrum:

$$
\bar{E}_{B j}(d)=P\left(d, E_{0}\right) \frac{\int_{0}^{E_{0}} E N(E) d E}{\int_{0}^{E_{0}} N(E) d E}
$$

where:

$$
\begin{aligned}
\bar{E}_{B j}(d) & \text { effective beta energy at a depth } d \text { in lissue } \\
& \text { for peak } i \mathrm{MeV} / \text { disintegration } \\
\mathrm{d} & \text { - exposure point tissue depth in } \mathrm{mg} / \mathrm{cm}^{2}
\end{aligned}
$$




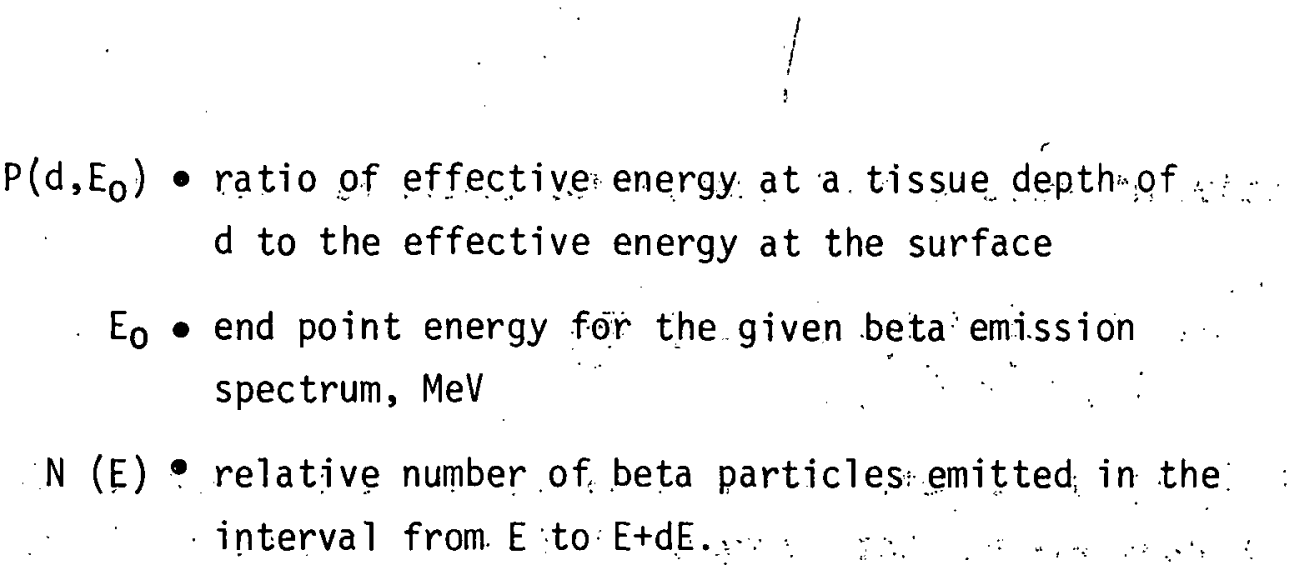

The total effective energy for a nuclide is the sum of the individual effective energies weighted by the peak abundance.

$$
\bar{E}_{B}(d)=\sum_{i=1}^{\substack{\text { Number of } \\ \text { Beta Peaks }}} E_{B i}(d) A_{i}
$$

where:

$A_{i}$ - beta particle abundance for peak $i$, particles/ disintegration.

A formulation of the beta energy reduction factor indicates that it is a function of tissue depth and beta end point energy. (4) Beta dose is proportional to beta energy (for the semi-infinite cloud assumption); thus the beta energy reduction factor is equal to the beta dose reduction factors.

The beta energy reduction factor is given by:

$$
P\left(d, E_{0}\right)=\frac{\bar{E}_{\beta}(d)}{\bar{E}_{\beta}(0)}=\alpha\left[A \delta^{2}+\exp (1-v d)\right]
$$

where:

$$
\begin{aligned}
& A=3-\exp (1-v d / \delta)-(\nu d / \delta)(2+\ln \delta / v d) \\
& A=0 \text { for } d \geq \delta / v
\end{aligned}
$$

and: $\quad \nu$ effective attenuation coefficient for "a peak' with endpuinl energy $E_{0}, \mathrm{~cm}^{2} / \mathrm{mg}$

d tissue depth, $\mathrm{mg} / \mathrm{cm}^{2}$

$E_{0}$ - maximum energy of characteristic beta energy spectrum, MeV a, $\delta$ - empirical constants dependent on $\mathrm{E}_{0}$, dimensionless. 
The effective attenuation coefficient $v$ for tissue is given by:

$$
\nu=\frac{18.6}{\left(E_{0}-0.036\right)^{1.37}}\left(2-\bar{E}_{\beta} / \bar{E}_{\beta}^{\star}\right)
$$

The ratio $\bar{E}_{\beta} / \bar{E}_{\beta}^{\star}$ is the ratio of the actual average beta energy to the hypothetical allowed average beta energy. "This ratio is 1.0 for allowed emissions. Values of $\alpha$ and $\delta$ are given in the table below.

\section{Values for $\alpha$ and $\delta$}

$\begin{array}{lll}0.10 \leq E_{0} \leq 0.5 \mathrm{MeV} & 0.260 & \frac{\alpha}{2.0} \\ 0.5<E_{0} \leq 1.5 \mathrm{MeV} & 0.297 & 1.5 \\ 1.5 E_{0} \leq 3.0 \mathrm{MeV} & 0.333 & 1.0\end{array}$

For values of $E_{0}$ less than $0.10 \mathrm{MeV}$, the reduction factor is set to zero. For values of $E_{O}$ greater than $3.0 \mathrm{MeV}$, values of $\delta$ and $\alpha$ of 1 and 0.333 respectively are used with the actual value of $E_{0}$.

To determine the average beta energy for a characteristic spectrum, the shape of the peak must be known. For allowed spectra the shape is given by: $(5,6)$

$$
N(E) d E=K \cdot F(Z, W)\left(W^{2}-1\right)^{1 / 2} W\left(E_{0}-E\right)^{2} d E
$$

where:

$K$ - arbitrary constant which cancels out in Equation (16)

$F(Z, W)$ - Fermi function for emissions from an atom of atomic number $Z$

W - total energy of the beta particle in units of rest mass

E - kinetic energy of beta particle in units of rest. mass $(W=E+1)$ 


$$
Z=\text { a tomic mass number }
$$

Energy is converted to rest mass units by dividing the energy in MeV by 0.514 .

The Fermi function is evaluated as

$$
F(Z, W)=2(1+\gamma)(2 p R)^{2(\gamma-1)} \exp \left[\pi y \frac{|\Gamma(\gamma+i y)|^{2}}{\Gamma(2 \gamma+1)^{2}}\right]
$$

where:

$$
\begin{aligned}
& i=\sqrt{-1} \\
& \gamma=\left[1-(\alpha Z)^{2}\right]^{1 / 2} \text { (dimensionless). } \\
& \alpha=e^{2} / k c \approx i . / 137 \text {. (dimensionless): } \\
& \text { e elementary charge, esu } \\
& h=h / 2 \pi \\
& \text { h - Plank's constant, erg-sec } \\
& c \because \text { speed of light, } \mathrm{cm} / \mathrm{sec} \\
& y=Z W / p \text { (dimensionless) } \\
& R=1 / 2 \propto A^{1 / 3} \text {, nuclear radius, } \mathrm{cm} \\
& P=\left(W^{2}-1\right)^{1 / 2} \text {, beta: particle momentum } \\
& \text { A - atomic weight }
\end{aligned}
$$

The data libraries of computer program SUBDOSA include data on 331 fission products plus 144 activation products or transuranic elements. Effective beta energies have been calculated for each of these nuclides for tissue depths of $0,7,20$, and $100 \mathrm{mg} / \mathrm{cm}^{2}$. The results have been incorporated into a data library for use by SUBDOSA.

SKIN, EYE, MALE GONAD, AND TOTAL BODY DOSE

The consequences of accidental radioactive releases to the atmosphere are often determined in terms of dose to skin; eyes; male gonads and total 
body. The skin dose is the sum of the surface gammia dose and the beta depth dose at $7 \mathrm{mg} / \mathrm{cm}^{2}$ (thickness of outer skin). The eye dose is the sum of the surface gamma dose and the beta depth dose at $100 \mathrm{mg} / \mathrm{cm}^{2}$ (depth of lens). The dose at a tissue depth of $1 \mathrm{~cm}$ is representative of the dose to the male gonads. The total body dose as calculated as the gamma dose at a tissue depth of $5 \mathrm{~cm}$ with no beta contributions included. The computer program SUBDOSA has been designed for calculation of these special doses.

\section{NORMALIZED AIR CONCENTRATION}

The normalized air concentration has been described for gamma and beta dose calculations by Equations (3) and (14). These equations include a term for radioactive decay for a specific isotope. For hand calculation, values of $x / Q^{\prime}$ which do not include decay are desirable. Such values are calculated as:

$$
\frac{x}{Q^{\prime}}=\frac{\exp \left[-h^{2} / 2 \sigma_{z}\right]}{\pi \bar{u}_{h} \sigma_{y} \sigma_{z}}
$$

Plume reflection at the ground plane is included in Equation (21).

Methods for determining the dispersion parameters $\sigma_{y}$ and $\sigma_{z}$ include the Hanford equations, $(2,7)$ Sutton's equation, $(2,8)$ Brigg's equations $(9)$ and the curves attributed to Pasquil1. (2) The Hanford equations are:

$$
\begin{aligned}
& \sigma_{y}^{2}=A\left[T-\alpha\left(1-e^{-T / \alpha}\right)\right] \\
& \sigma_{z}^{2}=\alpha\left[1-\exp \left(-k^{2} T^{2}\right)\right]+b T
\end{aligned}
$$

where:

$$
\begin{aligned}
& t \cdot \text { duration of the release, sec } \\
& T \cdot \text { transport time from the point of release to the receptor, sec } \\
& A=\left[c+230\left(\sigma_{\theta} \bar{u}_{h}\right)\right] \\
& \alpha=A /\left[2\left(\sigma_{\theta} \bar{u}_{h}\right)^{2}\right.
\end{aligned}
$$

and $a, b, c, k$, and $\sigma_{\theta} \bar{u}_{h}$ are parameters whose values depend upon a tmospheric stability. 
The Sutton Model defines the variances as:

$$
\begin{aligned}
\sigma_{y} & =\frac{c_{y} x^{(1-n / 2)}}{\sqrt{2}} \\
\sigma_{z} & =\frac{c_{z} x^{(1-n / 2)}}{\sqrt{2}}
\end{aligned}
$$

where $C_{y}, C_{z}$, and $n$ are parameters whose values are dependent upon a tmospheric stability. Characteristic values of the parameters for the Hanford and Sutton equations are listed in Tables 1 and 2 , respectively.

Briggs relationships of $\sigma_{y}$ and $\sigma_{z}$, with distances for Pasquill Stability classes $A$ through $G$ are listed in Table 3 .

Graphical representations of $\sigma_{y}$ and $\sigma_{z}$ for the six Pasquill stability categories $^{(2)}$ have been tabulated and incorporated in a 1 ibrary for the computer code KRONIC ${ }^{(10)}$ (Tables 4 and 5 ).

TABLE 1. Values of Meteorological Parameters for the Hanford Model.

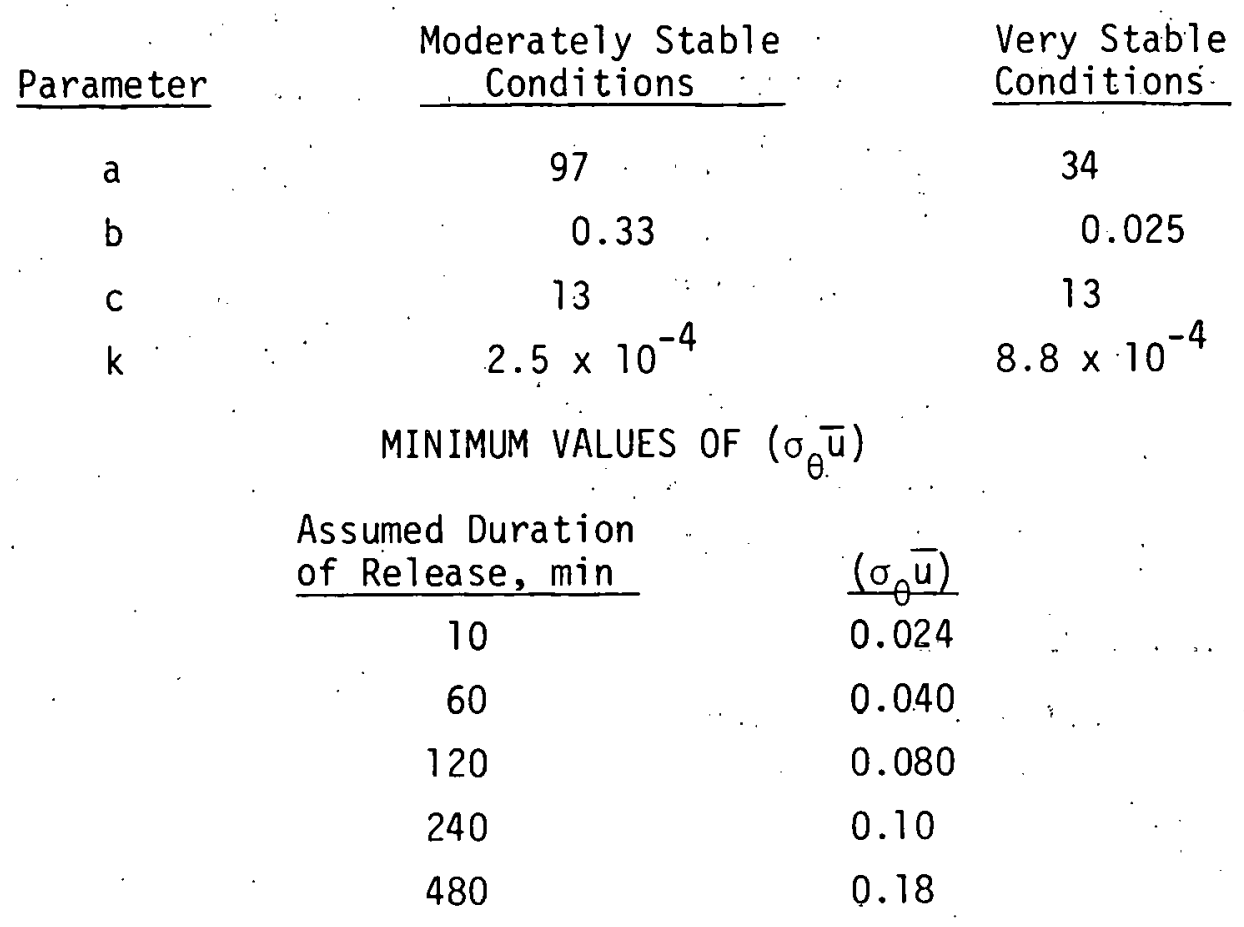


TABLE 2. Numerical Values of Atmospheric Dispersion Parameters. for Neutral and Unstable Atmospheres

\begin{tabular}{|c|c|c|c|c|c|}
\hline Paramet & ter & Release Level & Wind Speed & Unstable & Neutral \\
\hline$C_{y}$ & & Ground & $1 \mathrm{~m} / \mathrm{s}$ & 0.35 & 0.21 \\
\hline $\boldsymbol{J}$ & & & $5 \mathrm{~m} / \mathrm{s}$ & 0.30 & 0.15 \\
\hline & & & $10 \mathrm{~m} / \mathrm{s}$ & 0.28 & 0.14 \\
\hline & & Elevated & $1 \mathrm{~m} / \mathrm{s}$ & 0.30 & 0.15 \\
\hline & $\therefore$ & & $5 \mathrm{~m} / \mathrm{s}$ & 0.26 & 0.12 \\
\hline . & & & $10 \mathrm{~m} / \mathrm{s}$ & 0.24 & 0.11. \\
\hline$c_{2}$. & & Ground & $1 \mathrm{~m} / \mathrm{s}$ & 0.35 & 0.17 \\
\hline & & & $5 . \mathrm{m} / \mathrm{s}$ & 0.30 & 0.14 \\
\hline & & & $10 \mathrm{~m} / \mathrm{s}$ & 0.28 & 0.13 \\
\hline & & Elevated & $1 \mathrm{~m} / \mathrm{s}$ & 0.30 & 0.15 \\
\hline & & . & $5 \mathrm{~m} / \mathrm{s}$ & 0.26 & 0.12 \\
\hline . & & . & $10 \mathrm{~m} / \mathrm{s}$ & 0.24 & 0.11 \\
\hline$n$ & & & & 0.20 & 0.25 \\
\hline
\end{tabular}

TABLE 3. Relationships Between Diffusion Parameters and Distance.

\begin{tabular}{|c|c|c|}
\hline $\begin{array}{c}\text { Stability } \\
\text { Class } \\
\end{array}$ & $\begin{array}{c}\text { Horizontal } \\
\text { Parameter, } \sigma_{y}\end{array}$ & $\begin{array}{c}\text { Vertical } \\
\text { Parameter, } \sigma \\
\text { _._. }=\end{array}$ \\
\hline A & $220 x /(1+0.1 \times)^{1 / 2^{(a)}}$ & $200 \times$ \\
\hline$B$ & $160 x /(1+0.1 x)^{1 / 2}$ & $120 x$ \\
\hline$C$ & $110 x /(1+0.1 x)^{1 / 2}$ & $80 x /(1+0.2 x)^{1 / 2}$ \\
\hline 0 & $80 x /(1+0.1 \cdot x)^{1 / 2}$ & $60 x /(1+1.5 x)^{1 / 2}$ \\
\hline$E$ & $60 x /(1+0.1 x)^{1 / 2}$ & $30 x /(1+0.3 x)$ \\
\hline$\Gamma$ & $40 x /(1+0.1 x)^{1 / 2}$ & $20 x /(1+0.3 x)$ \\
\hline G & $27 x /(1+0.1 x)^{1 / 2}$ & $12 x /(1+0.3 x)$ \\
\hline
\end{tabular}

(a) $x$ is distance in kilometers 
TABLE 4. Values of $\sigma_{y}$ for Pasquill Stability Categories

Downwind Distance

(Meters)

100

250

500

1,000

2,500

5,000

450

830

A 21

B $\quad \sigma_{\text {C }} \frac{\text { for Pasquill Type }}{16} \quad-\mathrm{D}$

10,000

1,600

16

12

8.0

$\frac{E}{6.0} \cdot \frac{F}{3.9}$

25,000

54

40

28

20

14

9.8

50,000

3,400

76

55

37

28

18

$150 . \quad .110$

72

52

36

$340 \quad 240$

160

.120

81

630

450

310

220

150

$850 \div 570$

410

280

$100,000 \quad 11,000$

2,600

1,800

1,200

880

610

6,200
11,000

$4,700 \quad 3,400$

2,300

1,600

1,100

$8,500 \cdot 6,300 \cdot 4,100$

2,800

2,000

TABLE 5. Values of $\sigma_{z}$ for Pasquilil Stability Categories

Downwind Distance $\sigma_{z}$ for Pasquilit Types

\begin{tabular}{|c|c|c|c|c|c|c|}
\hline (Meters) & A & B & $C$ & $D$ & $E$ & $\therefore F^{\circ}$ \\
\hline $.100^{\circ}$ & 15 & 10 & 7.8 & 4.7 & 3.0 & 1.4 \\
\hline 250 & 43 & 26 & 18 & 10 & $\therefore 7.1$ & 4.0 \\
\hline 500 & 140 & 57 & 34 & 19 & 13 & 7.6 \\
\hline 1,000 & 670 & 140 & 64 & 33 & 22 & .14 \\
\hline 2,500 & 2,000 & 580 & 140 & 62 & 41 & 25 \\
\hline 5,000 & 2,000 & 2,000 & 260 & $: 95$ & 61 & 35 \\
\hline 10,000 & 2,000 & 2,000 & 440 & 140 & 84 & 47 \\
\hline 25,000 & $.2,000$ & 2,000 & 880 & 220 & 120 & 64 \\
\hline 50,000 & 2,000 & 2,000 & 1,400 & 320 & 140 & 79 \\
\hline 100,000 & 2,000 & 2,000 & 2,000 & 450 & 170 & 94 \\
\hline
\end{tabular}


The model.s described in the previous, section have been incorporated into a computer program for use on CDC CYBER 74 computers: The main program SUBDOSA is executable with or without two auxiliary programs, BELI and BIVAR.

$$
\begin{aligned}
& \text { BELI - a program for preparation of effective beta energy data. } \\
& \text { BIVAR - a program for calculation of dose rate factors for a } \\
& \text { bivariate plume. } \\
& \text { SUBDOSA - main program for calculating dose and normalized air } \\
& \text { concentrations. }
\end{aligned}
$$

A listing of the program is given in Appendix B. Input card preparation is described in Appendix $C$ and sample problems are $i 1 l$ ustrated in Appendix $D$. A program flow diagram is given in Appendix $E$.

\section{$\underline{B E L I}$}

The program BELI calculates effective beta energies for each nuclide as a function of tissue depth. Equations (15) through (20). Beta spectrum data for each beta peak (abundance and endpoint energy) is used as input. Appendix A describes input data for BELI and source references for the beta spectrum data.

BIVAR

The program BIVAR calculates dose rate factors by performing the space integration indicated in Equation (5): A modified version of subroutine PLUME from the computer program RACER is used to perform the numerical integration. (3) The actual integration is performed in the following order: $y$ direction (lateral), $z$ direction (vertical) and $x$ direction. The first two integrations are performed for each position in the $x$ direction (as located to give sufficient accuracy). The resulting double integral represents the dose rate per unit length of downwind plume per curie of a nuclide. The integration is performed for each energy group at each $x$ position. Integration in the $x$ direction then gives the dose rate factors. 
All integrations are performed by an eight point polynomial integration subroutine.

$$
\int_{x_{1}}^{x_{8}} f(x) d x=\frac{x_{8}-x_{1}}{7 c} \sum_{i=1}^{8} f\left(x_{i}\right) w_{i}
$$

where $C$ is 17,280 and $w_{i}$ are the integration weights as follows:

$$
\begin{aligned}
& w_{1}=w_{8}=751 \\
& w_{2}=w_{7}=3577 \\
& w_{3}=w_{6}=1323 \\
& w_{4}=w_{5}=2989
\end{aligned}
$$

Each integration range is divided into sections and Equation (24) is applied to each section. This technique allows computing time to be minimized without loss of accuracy.

The dose factors are automatically punched onto cards for future use. They may be used directly by the gamma dose calculation in the program. SUBDOSA or they may be incorporated into a dose rate factor library (Appen$\operatorname{dix}$ A) for direct access by the computer.

\section{SUBDOSA}

The ma in program SUBDOSA calculates gamma doses, beta doses, and normalized air concentrations. The calculations in the program are completed as indicated by Equations (1) through (14) or the previous section. However, several features are worth mentioning.

- The program uses as many as three data libraries (described in Appendix A):

1. Radionuclide data library - physical hälf lives, decay fractions, effective beta energy as a function of tissue depth

2. Gamma energy library - photon energies and abundance 
3. Gamma dose rate factors - as calculated by BIVAR for use in finite cloud gamma dose calculation

- Data is included for nearly 500 radionuclides which include fission products and activation products.

- Dose calculations are performed for each release period and summed to give the total dose after each successive period.

- Depth dose may be calculated for both gamma and beta exposure. Default values of tissue depths are 0,1 , and $5 \mathrm{~cm}$ for gamma doses and $0,7,20$, and $100 \mathrm{mg} / \mathrm{cm}^{2}$ for beta doses.

- Combinations of beta and gamma depth doses may be sưmmed to simulate: eye dose, skin dose, dose to male gonads and total body dose. The skin dose is the sum of surface tissue dose from $\therefore$ gamma radiation p.lus the dose at $0.007 \mathrm{~cm}$ depth in tissue from beta contributors. The eye dose is the sum of surface tissue dose from gamma radiation plus the dose at $0.1 \mathrm{~cm}$ in tissue from beta contributors." Both gonadal and total body doses neglect contributions from beta radiations. However, absorption of gamma radiation in tissue at the corresponding depthis is accounted for in the dose calculation. 


\section{REFERENCES}

1. Berger, M. J., Engineering Compendium on Radiation Shielding, Volume I, p. 218, Springer-Verlag New York, Inc., 1968.

2. Meteorology and Atomic Energy, D. H. Slade, editor, U.S. Atomic Energy Commission "Division of Technical Information, 1968.

3. Strenge, D. L., M. M. Hendrickson, and E. C. Watson, RACER -. A Computer Program for Calculating Potential External Dose from Airborne Fission Products Following Postulated Reactor Accidents, BNWL-B-69, June 1971.

4. Loevinger, R., E. M. Japha, and G. L. Brownell, "Discrete Radioisotope Sources," Chapter 16, Radiation Dosimetry, Academic Press, Inc., 1956.

5. Siegbahn, K., editor, Beta and Gamma Ray Spectroscopy, Interscience Publishers, Inc., 1955.

6. Marshall, J. H., "How to Figure Shapes of Beta-Ray Spectra," NUCLEONICS, Vol. 13, No. 8, August 1955.

7. Fuquay, J. J., C. L. Simpson, and W. T. Hinds, Estimates of Ground Level Air Exposures Resulting from Protracted Emissions from 70-Meter Stacks at Hanford, HW80204; 1964.

8. Sutton, 0. G., Micrometeorology, McGraw-Hill Book Company, 1953.

9. Briggs, G. A., Diffusion Estimation for Small Emissions, ATDL 106, December, 1974.

10. Strenge, D. L., and E. C. Watson, KRONIC - A Computer Program for Calculating Annual Average External Doses from Chronic Atmospheric Releases of Radionuclides, BNWL-B-264, June 1973. 
APPENDIX A

DATA LIBRARIES 


\section{APPENDIX A}

\section{DATA LIBRARIES}

This appendix describes data iibraries used by BELI and SUBDOSA. Input libraries for BELI are BISLIB (beta spectrum data) and RNDBET (radionuclide and beta energy data library). SUBDOSA uses RNDBET, GISLIB (photon data library) and BIVLIB (gamma dose rate factor library). A listing of each library is included at the end of this appendix.

\section{INPUT FOR BELI - BETA SPECTRUM DATA LIBRARY}

The program BELI uses libraries BISLIB and part of RNDBET to calculate effective beta energies for library RNDBET. BELI compares the nuclides of each library and calculates effective beta energies for nuclides present, in both libraries. For nuclides present only in RNDBET, effective energies of zero are entered (zeros are entered for only a few nuclides in the activation product section of the libraries).

Data of the beta spectrum library's BISLIB, include the maximum beta: energy for each peak and the fraction of total disintegrations for the peak. Also included at the beginning of the library are the literature sources for the data. A listing of BISLIB is given in Appendix $B$.

The beta spectrum data for each nuclide are contained on from. 1 to 1.5 cards. The first card for each nuclide has the following format:

\begin{tabular}{|c|c|c|}
\hline Column & Format & Variable \\
\hline $1-3$ & I3 & Atomic Máss Number \\
\hline $4-5$ & I2 & Átomic Number \\
\hline $6-7$ & A2 & Nuclide name \\
\hline 8 & I1 & Nuclide state: $\begin{array}{l}1 \text { - isömeric } \\
2-\text { ground }\end{array}$ \\
\hline $9-10$ & $\mathrm{I} 2$ & Number of beta peaks to be read \\
\hline $17-15$ & F5.0 & Abundance of first beta peak (if any) \\
\hline
\end{tabular}




\begin{tabular}{|c|c|c|}
\hline Column & Format & Variable \\
\hline $16-20$ & $\mathrm{~F} 5.0$ & Maximum energy of first peak, MeV \\
\hline $21-25$ & F5.0 & Abundance of second peak (if any) \\
\hline $26-30$ & F5.0 & $\begin{array}{l}\text { Maximum energy of second peak, MeV } \\
\therefore\end{array}$ \\
\hline $\begin{array}{l}\bullet \\
:\end{array}$ & & 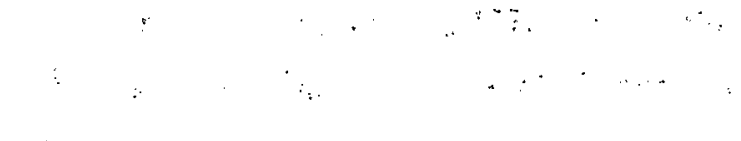 \\
\hline $61-65$ & $\mathrm{~F} 5.0$ & Abundance of sixth peak (if any) \\
\hline $66-70$ & $\mathrm{~F} 5.0$ & Maximum energy of sixth peak, MeV \\
\hline 76 & I1 & $\begin{array}{c}\text { Spectrum type: blank - allowed } \\
.1-\text { unallowed }\end{array}$ \\
\hline
\end{tabular}

If the nuclide has more than six beta peaks, additional cards are needed. The format for additional cards is 14F5.0, with the abundances and maximum: beta energies submitted in pairs (for up to seven peaks) as: on the first card but starting in column 1. A maximum of 99. peaks may be supplied for each nuclide (15 cards).

\section{RADIONUCLIDE AND BETA ENERGY DATA LIBRARY:}

This data library contains data on radionuclide decay schemes and half-: lives and beta energy as a function of tissue depth. Program BELI only considers the list of nuclides and ignores the physical data. Program SUBDOSA uses some of the half-rives and decay scheme data.

The order of isotopes in this library determines the order for which data are supplied in the photon probability library. When the order of data is changed in the radionuclide data library, corresponding changes must be made in the photon probability library.

The first card image of the radionuclide data library contains the number of fission products in the library. (format 13). There is one card for each fission product. The cards are arranged in an ascending atomic number order within an ascending atomic mass sequence. The current radionuclide data library contains 331 fission products. 
The card after the last fission product card contains the number of activation products in the library (Format I3). The term "activation product" includes all radionuclides that are not in the fission product portion. of the library. There is one card per activation product. ' The current library contains 144 activation products.

The format for each radionuclide card is:

\begin{tabular}{|c|c|c|}
\hline Column & Format & Variable \\
\hline $1-3$ & I3 & Atomic Mass Number \\
\hline $4-5$ & 12. & Atomic Number \\
\hline $6-8$ & A3 & $\begin{array}{l}\text { Nuclide symbol with isomeric states } \\
\text { indicated by an asterisk; i.e., } \mathrm{KR}^{\star} \text {, } \\
\text { left justified. }\end{array}$ \\
\hline 9 & I1 & $\begin{array}{l}\text { Interger to indicate isomeric state: } \\
1 \text { - isomeric state } \\
2 \text { - ground state }\end{array}$ \\
\hline $10-18$ & E9. 2 & Physical half-life in days \\
\hline $19-25$ & $F 7.4$ & $\begin{array}{l}\text { If in isomeric state, fraction. of } \\
\text { decays by beta emission; if in ground } \\
\text { state, fraction of decays to an isomer }\end{array}$ \\
\hline $26-35$ & F10.6 & $\begin{array}{l}\text { Effective beta energy per disintegra- } \\
\text { tion, MeV }\end{array}$ \\
\hline $36-45$ & F10.6 & $\begin{array}{l}\text { Effective beta energy per disintegra- } \\
\text { tion corrected for penetration of } \\
7 \mathrm{mg} / \mathrm{cm}^{2} \text { of tissue, } \mathrm{MeV}\end{array}$ \\
\hline $46-55$ & F10.6 & $\begin{array}{l}\text { Effective beta energy per disintegra- } \\
\text { tion corrected for penetration of } \\
20 \mathrm{mg} / \mathrm{cm}^{2} \text { of tissue, MeV }\end{array}$ \\
\hline $56-65$ & F10.6 & $\begin{array}{l}\text { Effective bela encrgy per disintegra- } \\
\text { tion corrected for penetration of } \\
100 \mathrm{mg} / \mathrm{cm}^{2} \text { of tissue, MeV }\end{array}$ \\
\hline
\end{tabular}

The radionuclide library available with SUBDOSA is called RNDBET and is listed in Appendix B.

\section{PHOTON PROBABILITY LIBRARY}

The photon probability library contains data for use by subroutine SUBDOSA in calculating the cloud gamma dose. The first card image contains 
the number of energy groups (Format. I3) for which data are given in the library. The second card contains the upper energy bound for each group (Format 16F5.0) starting from the lowest energy group (number of entries is. number of groups minus one). The next cards contain dose conversion factors in units of:

$$
\frac{\mathrm{rad} \cdot \mathrm{cm}^{2}}{\mathrm{Ci} \cdot \mathrm{sec}}
$$

One value is supplied for each energy group starting from the lowest energy. The dose conversion factor of Equation (1) in the tcxt is the library value divided by the average energy for the group. "The average-energy is calculated as the midpoint of maximum energies as supplied on the second library card. The average energy for the first group is, $2 / 3$ of its maximum energy and the average for the last energy group is 1.25 times the maximum for the next lower group.

The photon probability and gamma energy cards for each nuclide follow the dose conversion factor cards. There are from one to three cards for each nuclide (in the order of nuclides as in the radionuclide data library). The first card for each nuclide has the following format:

\begin{tabular}{|c|c|c|}
\hline Column & Format & Variable \\
\hline $1-3$ & 13 & Atomic Mass Number \\
\hline $4-5$ & 12 & Atomic number \\
\hline 6.7 & A2 & Nuclide name $\quad \therefore$ \\
\hline 8 & Il & Nuclide state: $\begin{array}{r}1 \text { - isomeric } \\
2 \text { - ground }\end{array}$ \\
\hline $9-10$ & I2 & Number of photon energies to be read \\
\hline $11-15$ & F6.0 & Aburidance of first photon (if any) \\
\hline $16-20$ & F5.0 & Energy of first photon, MeV. \\
\hline $21-25$ & F5.0 & Abundance of second photun ( $i f$ dny). \\
\hline $26-30$ & F5.0 & Energy of second photon, MeV \\
\hline • & & $\therefore$ \\
\hline$\cdot$ & & $\because, \cdots, \ldots, \cdots \cdot \cdots$ \\
\hline $61-65$ & F5.0 & Abundance of sixth photon (if any) \\
\hline $66-70$ & F5.0 & Energy of sixth photon, MeV \\
\hline
\end{tabular}


If the nuclide has more than 6 photons, additional cards are supplied in Format 14F5.0 with abundances and photon energies submitted in pairs (for up to 7 photons/card) as on the first card. A maximum of 99 photons may be supplied for each nuclide (1.5 cards)." Similarities are noticeable in the data sections of the photon probability library and the beta spectrum 1 ibrary.

Buildup and attenuation data are stored after the photon data. Constants for the quadratic buildup equation [Equation (2) in text] are given next in Format 8F10.0. The first card gives values for A for each energy group (if there are more than eight groups, more cards are needed). Next the values of $\alpha$ are given, followed by values of $\alpha_{1}$ (a dummy variable) and values of $\mu$ last (total mass attenuation coefficient in air, $\mathrm{cm}^{2} / \mathrm{g}$ ).

The photon probability library available with SUBDOSA is called GISLIB and is listed in Appendix B. The literature data sources for GISLIB are listed near the beginning of the library. The data sources for nuclides with a source code of 0 (for other) are listed in Table $A-1$ by nuclide number (Columns 77-79 in GISLIB).

TABLE A-1. Photon Data Literature Sources

\begin{tabular}{|c|c|}
\hline $\begin{array}{l}\text { Nuclide } \\
\text { Number } \\
\end{array}$ & Photon Data Source \\
\hline 060 & $\begin{array}{l}\text { Nuclear Abstracts, Abstract \#8968, p. } 915 \\
\text { (March 15, 1969) }\end{array}$ \\
\hline 074 & Nuclear Physics A98 337-64 (1967) \\
\hline 103 & Nurlear Data Sheets, Section A (Feb. 1968) \\
\hline 116 & Physical Review 154: 1116-25 (Feb. 20, 1967). \\
\hline 118 & Nuclear Data Sheets - Section A (Feb. 1968) \\
\hline 119 & Arkir för Fysik 34: 259-62. (1967) \\
\hline 129 & Nuclear Physics A109: $369-79(1968):$ \\
\hline 152 & Nuclear Physics 75: .209-14 (1965) : : \\
\hline 155 & Physical Review 146: $883-6$ (June 17 ; :1966) \\
\hline 159 & Nuclear Physics 75: $209-14:(1965)$ \\
\hline 165 & Radiochina Aeta 7: 114-15 (June 1967) \\
\hline 168 & $\begin{array}{l}\text { Physical Review 140B 1516-28 (Dec.:20, 1965) } \\
\text { The Handbook of Chemistry and Physics }(1964-5) \text {, } \\
\text { pp. E74-5 }\end{array}$ \\
\hline
\end{tabular}




\section{TABLE A-1. (Cốnt'inued)}

Nuclide

Number

17.2

182

191

194

$195^{\circ}$

196

201

242

249

259

201

265

269

277

279

288

289

308

309

315

316

319

334

343

345

347

348

350

358

379
Photon Data Source

Nuclear Physics A1.03: $385-405$ (1967)

Physical. Review 143: :918-22 (March 18, 1966)

Nuclear Data Sheets - Section A (Feb. 1968)

Nuclear Physics A126: 273-99 (1969)

Nuclear Physics A126: 273-99. (1969)

Nuclear Data Sheets - Section A (Feb. 1968)

Nuclear Physics A122: "\$57-566 (1968)

Nuclear Data Sheets - Section A (Feb. 1968)

Nuclear Phys1cs A113: 581-92 (1968)

Info. comes from article referenced in 1968 Vol of Nuclear Abstracts

Nuclear Data Sheets - Section A $1:: 527-602$

(August 1966)

Nuclear Physics A124: 199-211 (1.969)

Nuclear Physics A126: 428-30 (1969)

Nuclear Physics A99: 547-76 (1967)

Canadian Journal of Physics $44:-313-20$ (June 1966)

Nucleàr Physics A123: 481-96 (1969)

Nuclear Physics 1123: 481-96. (1969)

Physicd Review 172: 1253-61 (Aug. 20, 1968)

Physical Keview 172: 1253-61 (Aug. 20, 1968)

Airkir för Fysik 37: 203-12 (1968)

Airkir för Fysik 37: 203-12 (1968)

Canadian Journal of Physics 46: 2579-88

(Dec. 1, 1968).

Arkir för Fysik 37: 1-11: (1:968)

Arkir för Fys1k 34: 447-57: (1967).

Nuclear Physics A119: '53-64 (1968)

Physical Review 167: 1105-16 (March 20, 1968)

Physical Review 167: 1105-1:6 (March 20, 1968)

Nuclear Physics A113: 33-56 (1968)

Physical Review 167: 11.05-16 (March 20, 1968)

The Handbook of Chemistry and Physics (1964-5),

E 74-5 
TABLE A-1. (Continued)

Nucl ide

Number

382

391

3.93

403

417

420

425

437

438

440

452

478

492

493

494

496

498

499

500

\section{Photon Data Source}

The Handbook of Chemistry and Physics (1964-5), E 74-5

Nuclear Data Sheets - Section A (Feb. 1968)

The Handbook of Chemistry and Physics (1964-5), E 74-5

The Handbook of Chemistry and Physics (1.964-5), E 74-5

The Handbook of Chemistry and Physics (1964-5), E 74-5

Nuclear Physics A123: 1-23 (1969)

Nuclear Physics 82: 614-24 (1966)

Physical Review 147: 845-52 (Juily 22, 1966)

Phyșical: Review 153: 1310-11 (Jan. 20, 1967)

Physical Review 153: 1262-9 (Jàn. 20, 1967)

Nuclear Physics A118: 78-96 (1968)

Nuclear Physics 84: 424-42 (1966)

Physical Review 175: 1275-82 (Nov. 20, 1968)

The Handbook of Chemistry and Physics (1964-5)

E. 74-5

Nuclear Data Sheets - Section A (Feb. 9,:1968)

Nuclear Data Sheets - Section A (Feb. 1968)

Nuclear Data Sheets - Section A (Feb. 1968)

Nuclear Physics A84: 481-504

Nuclear Data Sheets - Section A (Feb. 1968)

Nuclear Data Sheets - Section A (Feb. 1968)

Nuclear Data Sheets - Section A (Feb. 1968)

Notes: $171\left({ }^{10} 6 M_{R H}\right)$ The info on the first $12, \gamma$-rays came from Table 1 of The Table of Isotopes. The last two $\gamma$-rays seem to have come from some source that could not be located.

$402\left({ }^{151} \mathrm{Pm}_{67}\right)$ In The Tabie of Isotopes much di sagreement over the probabilities attached to the emission of certain $\gamma$-rays by this isotope. Therefore, the probabilities listed are very uncertain. 


\section{DOSE RATE FACTOR LIBRARY}

The dose rate factor 1 ibrary: is for supplying dose rate factors, DRF, as described by Equation (5) of the text. The factors are calculated by program BIVAR.

Data for this library are supplied in sets with each set containing DRF values for one release height, one wind speed and up to ten distances. The first card of the library gives the number of sets contained in the library (Format I5). The DRF sets follow with the structure indicated below:

\begin{tabular}{|c|c|c|c|}
\hline Card & Column & Format & Variable/Use \\
\hline \multirow[t]{4}{*}{1} & $1-10$ & F10.2 & HD, height of release for this DRF set. \\
\hline & $11-15$ & I5 & $\begin{array}{l}\text { MD, number of meteorological stability cate- } \\
\text { gories. This variable was included for } \\
\text { future expansion only.., MD must be } 1 .\end{array}$ \\
\hline & $16-20$ & I5 & $\begin{array}{l}\text { MRD, number of distances for which DRF values } \\
\text { are supplied, maximum is } 10 . \text { : : }\end{array}$ \\
\hline & $21-25$ & 15 & $\begin{array}{l}\text { IEN, number of energy groups for which DRF } \\
\text { values are submitted. This number must equal } \\
\text { the number of energy groups as supplied on } \\
\text { the first card of the photon probability } \\
\text { library. The current number is } 12 \text {. }\end{array}$ \\
\hline 2 & $\begin{array}{l}1-10 \\
10-20 \\
\quad: \\
.\end{array}$ & $8 \mathrm{~F} 10.2$ & $\begin{array}{l}\text { RD, downwind distances (meters) for which DRF } \\
\text { values are given in this set. The number of } \\
\text { values submitted must correspond to MRD above. } \\
\text { If MRD is greater. than } 8 \text {, two cards are needed. }\end{array}$ \\
\hline 3 & $1-10$ & $8 F 10.2$ & $\begin{array}{l}\text { DRF values for a given release time and dis- } \\
\text { tance. One DRF value is given for each energy } \\
\text { group. Because the current-number of energy } \\
\text { groups is } 12,2 \text { cards are needed for each } \\
\text { distance/release period combination. }\end{array}$ \\
\hline
\end{tabular}

The number of cards of Type 3 needed depends on values given for MRD and a 1 so IEN, whenever IEN is greater than 8 (and less than 16).

The dose rate factor library supplied with SUBDOSA is called BIVLIB and is listed in Appendix. B. The dose rate factor sets included in BIVLIB are 1 isted in lable A-2 in order. The dose rate factor sets may be called by number for each release period as desired when doing the gamma dose calculations. 
TABLE A-2. Dose Rate Factor Sets in BIVLIB $(a)$

\begin{tabular}{|c|c|c|c|}
\hline $\begin{array}{l}\text { Set } \\
\text { No. }\end{array}$ & $\begin{array}{c}\text { Release } \\
\text { Height, m }\end{array}$ & $\begin{array}{c}\text { Wind Speed } \\
\mathrm{m} / \mathrm{sec}\end{array}$ & Dispersion Model \\
\hline 1 & 0 & 1 & Pasquill F \\
\hline 2 & 0 & 1 & Pasquill E \\
\hline 3 & 0 & 1 & Pasquill D \\
\hline 4 & 0 & 1 & Pasquill C \\
\hline 5 & 0 & 1 & Pasquill B \\
\hline 6 & 0 & 1 & Pasquili A \\
\hline 7 & 0 & 1 & Sutton unstable \\
\hline 8 & 0 & 1 & Sutton neutral \\
\hline 9 & 0 & 1 & Hanford very stable, $\sigma_{\theta} \cdot \bar{u}=0.024$ \\
\hline 10 & 0 & 1 & Hanford moderately stable, $\sigma_{\theta} \bar{u}=0.024$ \\
\hline 11 & 0 & 5 & Hanford moderately stable, $\sigma_{\theta} \bar{u}=0.024$ \\
\hline 12 & 0 & 10 & Hanford moderately stable, $\sigma_{\theta} \bar{u}=0.024$ \\
\hline 13 & 0 & 1 & Hanford moderately stable, $\sigma_{\theta} \bar{u}=0.04$. \\
\hline 14 & 0 & 5 & Hanford moderately stable, $\sigma_{\theta} \bar{u}=0.04$ \\
\hline 15 & 0 & 10 & Hanford moderately stable, $\sigma_{\theta} \bar{u}=0.04$ \\
\hline 16 & 0 & 10 & Hanford moderately stable, $\sigma_{\theta} \bar{u}=0.18$ \\
\hline 17 & 10 & 1. & Hanford moderately stable, $\sigma_{\theta} \bar{u}=0.024$ \\
\hline 18 & 10 & 1 & Hanford moderately stable, $\sigma_{\theta} \bar{u}=-0.04$ \\
\hline 19 & 10 & 1 & Hanford moderately stable, $\sigma_{\theta} \bar{u}=0.06$ \\
\hline 20 & 60 & 1 & Hanford moderately stable, $\sigma_{\theta} \bar{u}=0.024$ \\
\hline 21 & 60 & 5 & Hanford moderately stable, $\sigma_{\theta} \bar{u}=0.024$ \\
\hline 22 & 60 & 10 & Hanford moderately stable, $\sigma_{\theta} \bar{u}=0.024$ \\
\hline 23 & 60 & 1 & Hanford moderately stable, $\sigma_{\theta} \bar{u}=0.04$ \\
\hline 24 & 60 & 5 & Hanford moderately stable, $\sigma_{\theta} \bar{u}=0.04$ \\
\hline 25 & 60 & 10 & Hanford moderately stable, $\sigma_{\theta} \bar{u}=0.04$ \\
\hline
\end{tabular}

(a) All of the above sets contain data for the djstances (meters): 100 , $200,500,10^{3}, 2 \times 10^{3}, 5 \times 10^{3}, 10^{4}, 2 \times 10^{4}, 5 \times 10^{4}$, and $10^{5}$. 
APPENDIX B

PROGRAM LISTING 


\section{PROGRAM LISTING}

This appendix provides a listing of the computer program with information useful to the experienced programmer who may wish to modify the program.

The subroutine and function calling sequences for each of the three parts (BELI, BIVAR, and SUBDOSA) are shown in Figures. B-1. and B-2. BIVAR and SUBDOSA both use subroutines called PASSIG. These subroutines are not the same and should not be interchanged:

The purpose of each program, subroutine, and function is described below:

BELI Program to control calculation of effective beta energies at specified tissue depths

( $D$ in statement 12 of BELI).

BLIBE Subroutine to read library (BISLIB) of beta end point energies.

LIB Subroutine to read library (RNDBET) of nuclides to be included in output.

SIM3NI Function to perform Simpson $3 / 8$ rule numerical integration of energy of the shape of the beta spectrum as defined by function FERMI.

FERMI Function to calculate rèlative abundance of beta particles of a given beta energy for the spectrum being considered.

GAMMA Function for evaluation of the Gamma function.

DEPTH Function to calculate the depth dose reduction factor for a particular beta spectrum.

BIVAR Program to control calculation of dose rate factors. Input data is read; subroutine PLUME is called to calculate dose rate as a function of distance (x direction); and integration over distance is performed to give total dose rate factors.

FINT Function for integration according to Bode's rule for integration of order 2 through 7. A17 integrations in BIVAR and PLUME are seventh order (8 points). 

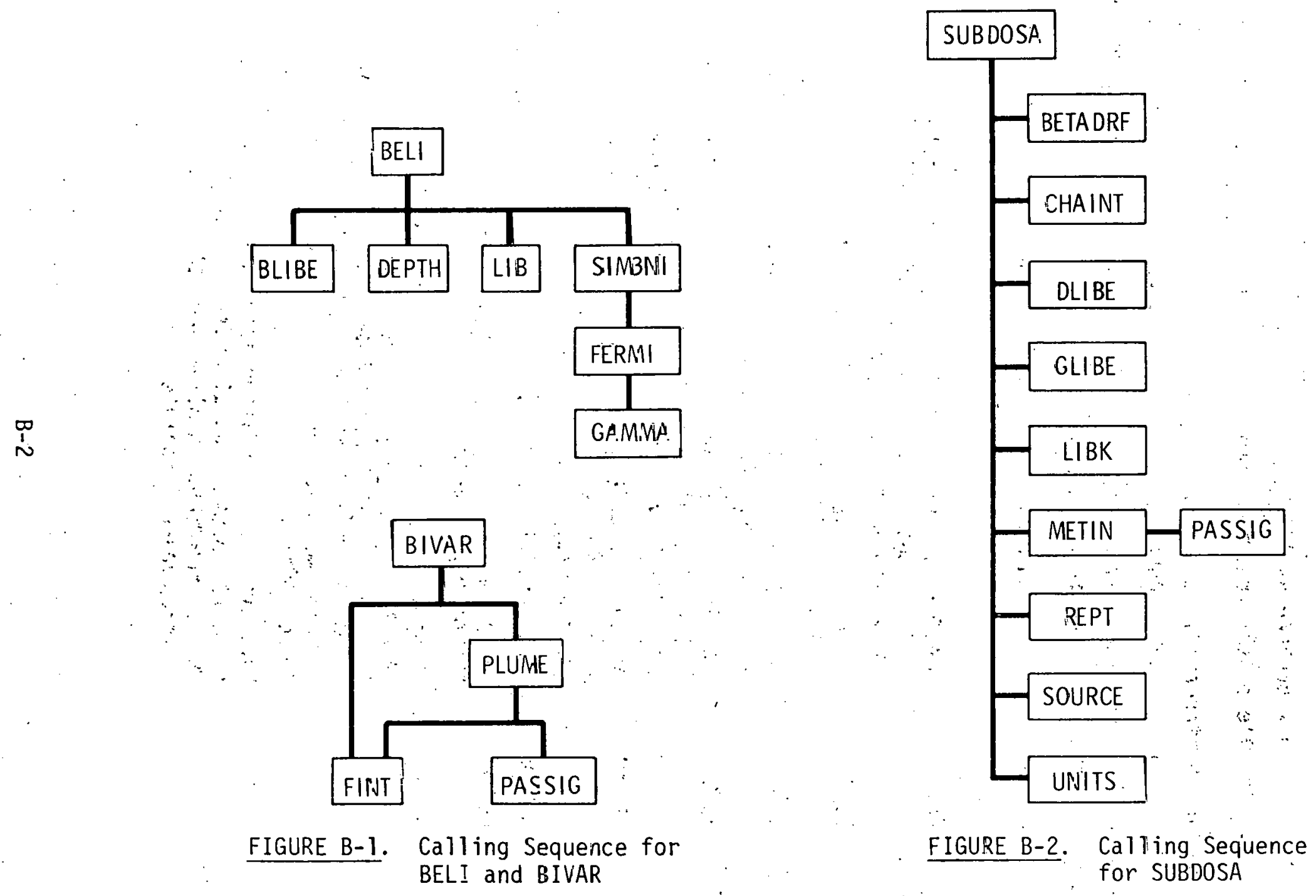


\begin{tabular}{|c|c|}
\hline PLUME & $\begin{array}{l}\text { Subroutine to perform dose rate factor inte- } \\
\text { gration in lateral }(y) \text { and vertical ( } z) \\
\text { directions. }\end{array}$ \\
\hline PASSIG & $\begin{array}{l}\text { Subroutine to determine standard deviation } \\
\text { of cloud concentration in lateral and verti- } \\
\text { cal directions ( } \sigma_{y} \text { and } \sigma_{z} \text { ) as a function of } \\
\text { distance for Pasquill dispersion categories. }\end{array}$ \\
\hline SUBDOSA & $\begin{array}{l}\text { Program to control and perform individual } \\
\text { beta and gamma dose calculations. }\end{array}$ \\
\hline BETADRF & $\begin{array}{l}\text { Subroutine to calculate beta dose rate } \\
\text { factors for each distance, meteorological } \\
\text { condition and release period specified. }\end{array}$ \\
\hline GLIBE & $\begin{array}{l}\text { Subroutine to read photon production data } \\
\text { library from input file GAMLIB. }\end{array}$ \\
\hline DLIBE & $\begin{array}{l}\text { Subroutine to read dose rate factor data } \\
\text { library from input file DRFLIB. }\end{array}$ \\
\hline LIBK & $\begin{array}{l}\text { Subroutine to read nuclide physical data } \\
\text { from input file RNDLIB. }\end{array}$ \\
\hline $\begin{array}{ll}\text { CHAINT: } \\
\vdots \\
\therefore & \vdots \\
\therefore & \vdots\end{array}$ & $\begin{array}{l}\text { Subroutine to perform fission product nuclide } \\
\text { decay calculation by decay chains. This sub:- } \\
\text { routine is also used to determine the time } \\
\text { integral of release when release is to inclüde } \\
\because \text { decay during the release period (ITZ }=0 \text { or: } 1 \text { ). }\end{array}$ \\
\hline METIN & $\begin{array}{l}\text { Subroutine to read atmospheric stability } \\
\text { (Types } 8-10 \text { ) and calculates stability para } \\
\therefore \sigma_{y} \text { and } \sigma_{z} .\end{array}$ \\
\hline PASSIG & Subroutine. (Same as PASSIG above.) \\
\hline REPT. & $\begin{array}{l}\text { Subroutine to prepare output reports and } \\
\text { punched card output. }\end{array}$ \\
\hline SOURCÉ & $\begin{array}{l}\therefore \text { Subroutine to read nuclide inventory cards: } \\
\because \text { (Card Types } 4-6 .)\end{array}$ \\
\hline UNI & $\begin{array}{l}\text { to determine units of release time } \\
\text { reports. }\end{array}$ \\
\hline
\end{tabular}

The programs, subroutines, and functions arë listed: in Figures B-3

through B-9. The order of the listings is the same as the order given in Figures B-1 and B.2. Data libraries follow the program listings in the order:

$\begin{array}{ll}\text { BISLIB } & \text { (Beta spectrum data for BELI) } \\ \text { RNDBET } & \begin{array}{l}\text { (Radionuclide and beta energy data library for BELI and } \\ \text { SUBDOSA) }\end{array} \\ \text { GISLIB } & \text { (Photon data library for SUBDOSA) } \\ \text { BIVLIB } & \text { (Gamma dose rate factor library for SUBDOSA) }\end{array}$


1

5

PROGRAM BELI (PUNCH, OUTPUT, BETLIA, RNDLIB, TAPEGEOUTPUT, TAPESEBET. IIR, TAPEST $=$ RNOL IT)

OTMENSION AVE (1D1)), EM(100), FF(100)

UIMENSION IARY(S)

DIMEVSION A (2)

DTMENSTION RFAC(4.100), EF(4)

LOGICAL NEL

TIVTCER RFC.RECQ

CDUMON/BETAIISOS, O(U), ML (OOO), REC 600$)$, BETENS $(2,2000)$, I HESUS(OO

10).LEIS,LACT,NI, NZ, MLH:600), RECAI6OO), IFOR(DOD), LAB (6OO), RAT (6OO)

CJMAON ATIN, ATN IFORBO, EMAX, ALPHA, PI, GAM, ALYAM,RAD,XI

EXTEHIAL FERYI

DATA $0 / 0.07 \ldots 20.0100 .1$

CART $(x)=x \times(1.13$.

ILAY $(!)=0$

IAAY $(Z)=0$

CALL STSTEMC(IISI (ARY).

CALL LIB

CALL PLIEE

A1. $P H A=1.1137$.

$P_{i} i=3.1+15{ }^{\circ}$

$4 . \Delta \times 1 i=? 0$

$E Q=. \cap 01$

UEL $=$ TRUE.

$\Delta(1)=001$

$c$ LONU OAd FISSION PRONUETS

Dn Q ISO=1.LFIS

IC $C$ SHFT (OEC (ISD) , IB) AND, T78

IF (TC. E. . . TIE) 60 TO IOJ

IF(IC.E.J.SSB) GO PO 203

G) TO !

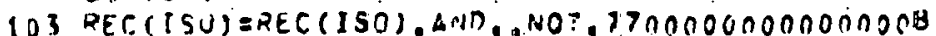
REC (ISI) $=$ QREC (ISO). OH. 34C00C0000C000008. (i) $10 \quad 303$

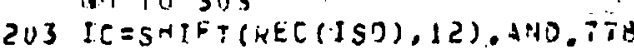

I.F (:C.EU. (19) GO YO. 403

IF $(: C . E B, S 5 H)$ GO TC 503

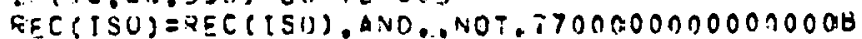

AER, ISO) =REC(ISO) . OR. $3500000000000000 B$

6010303

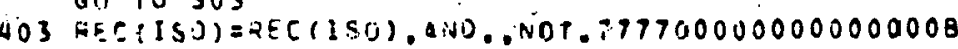

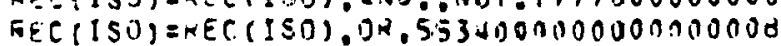
GO 10.303

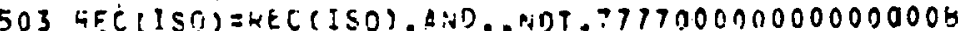

EEC (ISO) $=\nu E C([50), 02.5535 .900000000000007$

303 CONIINUE

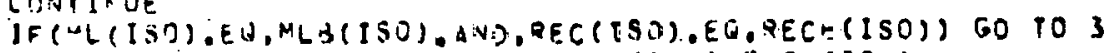

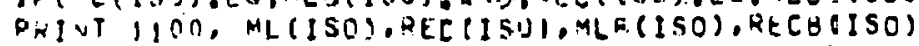

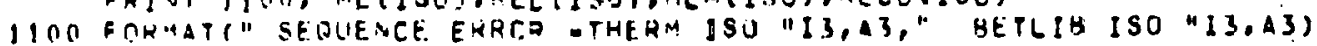

3 IIEHTN=IHESUH (ISO+1)-IEES JH(ISO)

EFF $=0$.

DO $104 \quad 1=1.4$

AF $(1)=0$. 
PROREAM BFLt.

$7373 \quad 001=1$

log Contritie

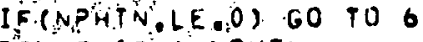

$R \triangle D=5 . A L P H A * C B A T(A T W)$

$x_{1}=2, \because G \Delta+1$

JF(IFORGO.LE.O) RAT (ISO) $=1.0$

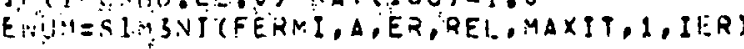

CONTIVUNE

EFF $=E$ A VE $\star F+E F P$

5 CIIATINUE

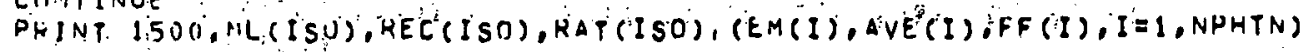
1) 1 ()

500 FORMAT" ISOTUDE "[.3,A3," ENERGY RATIO ="F5,2/(" "3(2F8,4,F8,5,6X)

1))

QF $(1)=E F F$

จั

SOT TO

(6) 0 , $\rightarrow: \mathrm{J}=1$

$B F(1)=0$.

7 Contintie

C PRINTIPUNCH RESULTS FOR CURRENT FISSION PROOUCT

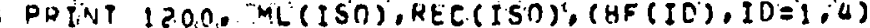

PUNem 1300, ML (ISO), REC(ISO), (BF (I0), IU=1, 4$)$

120.0 FRQMAT(IX.I3., $33.4 F 10.6)$

1300 FoRTIAT $(13,43,4 F 10,6)$

Q CoNTINIJE

C PRINTTRLVK ACTIVATION PRODUCT LIBRARY

DO 17, ISOENI, N2

DO 10 ID $=1,4$

EF $(10)=0$

105

1. CGNT INUTE

$I C=S H I^{K} T(R E C(1.5()), 1,8), \triangle H D, 77 H$

IF (IC.EO.47B) Gí TO li 
IF (IC.EP.558) GO TJ 21

Ge iो 1

111 REC (ISO) =REC (ISO) AHO NOT T7000OCA $10000000 B$

P.EC IISO) ₹PEC (ISO):DR. 34000000000001008 $G(1)$ In 311

211 TC $=5+I F T(R E C(1 S O), 129$, LND,TTE

IF (IC.E(.075) Go in a :

IF(IC.E.S.:558) GU 10051

115

REC (ISO) EREC (ISU). ANO. N(IT.77000000.10000000B

DEC (IS(I) =FEC (ISC). DP. I.50000000000017000 Gก T? 311

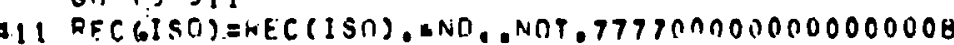

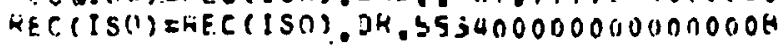
Gก 10 3is

511 QECCIS(1) = EEC (ISC) ENE NCT T777000000000000VOR AEC ISU $=$ HEC (ISO). LIR. $5535000.0000000000 B$

11 CONT THUE

DO II ISSENI. ISOS

125

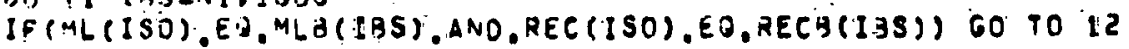

II CONTINUF.

- Dint laDo, mL (ISOY,REC(ISD)

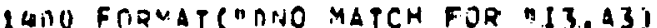

16 PfíT 12J0, ML (ISU),REC(ISO), (BF $(10), 10=1,4)$

PIJNCH 1300, MLC (ISO:,REC (ISO), (BF (IO), IO=1,4)

60 in 17

2 NRS I I S

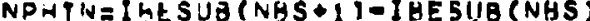

DC $13 \quad I=9,4$

$B F(t)=0$.

135

13 CNNTIPAIVE

IF(NPHTN,LE.O) GO TJ IS

EFE $=0$

DQ $15^{\circ} I B=1$, NPMTH

140

IRT =I BES IIH (NBS) - I

IFUOAO =IFOR (NBS)

EMAX = RETENS. (2, IBT + TR.)

IFIF"AX.LE, I.E-20) $60=015^{\prime}$

EeEMA $x$

145

$=E x A x$

$F=Q T E T E N S(1, I B T+I B)$

$F F($ I $)=F$

$A(?)=E M A X$

ATN=FLOAT (LAH (NHS)

150

ATEFLOAT (ML (AHES)

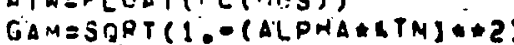

LI YAM =AL DHA.ATN

PADES S*ALPNAACRRT (ATW)

$M=2 . A G A M+1$.

Hat $(V A S)=1.0$

ENIJMESTISTNI LFERM

IF (IER) OK. 214

2)

IG (IET) $Q 9,31$ 
314. EAVE JFNUM/EOENOMMRAT (NRS)

$A V E(I H)=E \triangle V E$

Dii $14 . I=2,4$

SFAC (II,IB) $=0 E P T H(E, O(I), I F O K(N B S), I$ IOO)

BF (I): $=\forall F(I)+F$ Q AFAC $(I, I B)$ Q $A V E$

165

14 CUMTIINAE :

SE (1) =BF (1) +F*EAVE

i5. CANTtIIUE

PRINT 15NO, ML(ISO), REC(ISO), RAT ISO: , (EM(I), AVE (I), FF(I),IEI,NPHTN)

1)

170

lin ro is

17 Continue

1 P.PIN 100O, ISO,TC,REC (ISO),MLCISO)

1009 FOQM TA" CHAR. IDENT ERROR, ISO CHAR, REC,ML, EN4(020, (X))

จQ 5 ST

175

QR DFIV VIT $500, A T N A T W$

SOO FORNOTT" EHFOK IN SIMZNI. FOR ATOMIC NUMBER" FS.O," ANO ATOMIC HEIGHT

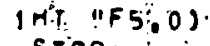

STDP

E 10 D 
1

c

5

20

SUBROUTINF. HLIBE

SUGGOUTINE TO READ THE GETA END POINT LIBRARY

INTEGER RECE,ANA

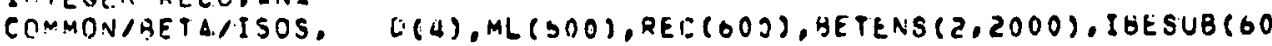

10) :LFIS,LAC.T, AI, NZ, MLE (OOO) , RECB $(00 D), I=O R(0 C O), L A B(600), R A T(600)$

$$
\text { gor to } 300
$$

400 OO $\triangle 01$ JJJE

II) READ (5, UCE) NCMATT

U.) FIRAAT (AI)

G:) 1025

300 REAT. $(5, ? 0)$ NPHEO, ISOS,NEMMT

20 FกQMAT (?I3, 6UX, I I:

IFCTSOS LE. (1) ISAS = NOHCD

IF (HCMMT. Gi. O) GU TO UJO

32 CONTINUE

SEP UP INUEXES FOR REAOING IM LIBE.

IRESIJP(1) $=1$

NOMTN $=1$

C DO LUOP IO READ. LIBE.

DO $100 \quad l=1$, :SOS

IENO = NPHTA 5

50 (1) 500

5.11 DI) $503 \mathrm{JJJ}=1, \mathrm{NCMMT}$

5 II REAO $(5,402)$ NCMMTY

500 (F 1905 D $L$ NPNTN) GO TO 60

REAC $(5,31)$ MASS, LATNO, ANA, NDC, (CEETENS $(J, K), J E 1,2), K=N P H T N$, IENO), 1 NCHAT, IFRD

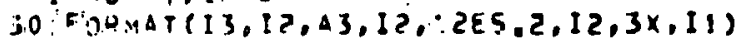

IFOR $(1)=I+60$

JF(NCMXY GT. O) GO TO 501

MENTN = NPHTN + NPC

"1PESUR $(I+1)=$ NPHTN

JE(TIPC AE O) GO TO 17

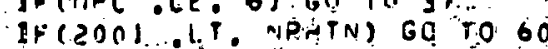

IAEGIN = RIOHTN + - NDC

IENO $=$ MIPHTN - I

AEAO $(5,3 b)$ ( (BETENS $(S, K), J=1,23, K=$ IBEGIN, IENO)

36 FRAMAT(1)ES.5)

37 CONTINUE

40 MLBCI $=$ MASS

LAHCI $=$ LATNJ

RECE(I) = ANA

45

100. CAnTINU

ENA LONP TORMEAD RE IA ENS POIMT LIAE

RETUAN

of $1.9=1-i$

WRITE $(0,70)$ i०

5n END 
5

FUNCTION OEPTHCEMAX,DD, JFORET, ISO

C THIS SUBROUTINE CALCULATES DOIDO FOR BHTA ENERGY EMAX AT A DEPTH OF C D MGICHZ IFGIIO GT O FOR FORBIODEN SIVECTRA. INTEGER RECE HEAL VIU

COMMOH/BETA/ISOS: $X(4), M L(600)$, REI: $(600)$, BETENS $(2,2000)$, IBESUB $(60$ 10), LFIS, LACT,NI, N2, MLE(OOO), RECA(601!), IFOK 600$), L A B(600)$, RAT (600) DITA RAT(55) 11.1

Data a

DATA PAT (TE)/1.1

DATA DAT (BG)/i.,

DATA hat $(90) / 1.1$

DATAO RAT(BZ) 1.17,

DATA EAT $(A \&) / 1.02 /$

DATA OAT(01)/1.04/

OATA RAT(O1)/1.03/

Q $110=1.0$

IF (IFUREO,GT:O) RATIOZRAT(ISO)

IF(RATIO.LE. 0,1) RATIOSI.0

RAT ISOS =2ATIO

IF (EMAX.LT..17) GO TO O

IF (EMAX.LY . S) 60 TO 3

IF (EMAX,LT, I.S) GO TO ?

IF(EMAX.GE.3.) GU TO 5

$1 \quad C=1 \cdot n$

$\triangle F H=.333$

Cic; in

2. $C=1.5$

$\Delta$ in $=297$

30

60106

$3 \mathrm{c}=\mathrm{z}$.

$A F=26$

Gก: 100

$3 \dot{5}$

100 FOAMATCH. GETA ENERGY = "F8,50" FOR ISOTOP "I3,A3)

IF(EMA.GP..1) GO 10 3

OSDTH=O

5 PHINT $200, E M A X, M L Q(I S O), R E C B(I S O)$

200 FORMATG HETA ENERGY"F5: I, "GREATER THAN 3. FOR ISOTOP "I3,A3) $G(10.1 \%$

$6: N\left(1=19.6 *(2.0-2.10) \int 0\right) /($ EMAX-.036)*1.37

$D N ! J=i+U=0 D+0.01$

$A=D N U / C$

$D=E \times D(1,-D N U)$

IF (A.CE, I.) GO TO T

$H=E \times P(1 ;-A)$

$C C=2 \cdot+A L O G(1.1 A)$

$B R A C=3,-B-A A C C$

50

G 10 OU

7 GR $A C=0$.

8. DEPTH $=\triangle D A \backsim(C+C \cap P R A C+D)$

RETULH

END 
SURROUTINE LIB

FTN 4.4\$2401

$07 / 07175 \quad 17.29 .02$.

PAGE

1.

1

5

10

15

20

$\stackrel{i}{0}$
SIJPROUTINE LIS

C THES SURPOUTINE READS A MASTER LIST OF NUCLIDE NAMES INTFGER REC

REAL LAMHOA. COMHON/3ETAIISOS, D(4), HL (600), REC 600$),$ BETENS $(2,2000)$, I BESUH 60

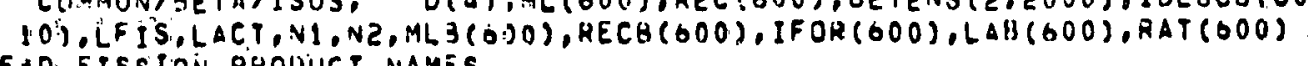

C REDO. ISSION PHOUUCT NAMES DE AD $(25,1)$ NCO

$\therefore$ FURMAT(I3)

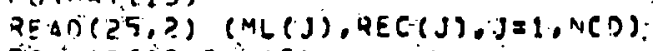

2 Figant $(13,2 \times, 43)$

C REA DCTIVATION PROOUCTS AND TRANSUAANI: NAMES PEAOC $(25,1) \mathrm{N} 2$

$N Y=N C B+1$

$N S=-12+M C D$

$N F P=N C O$.

$L F I S=N F P$

REAU $(25,2)$ (ML $(J), \operatorname{REC}(J), J=N I, N S)$

REMIVID 52

RETURN

En:D

$\therefore$ 
1

5

FUNCTIUN SIMSNI (FX,A,E, AEL, MAXIT,FK , IER)

C NIJMERICAL INTEGÜATION IJSING SIMPSUN.S J/B RULE.
SIMJNI

C.0. NiJMERICAL INTEGNATION IJSING SIMPSUN.S $3 / 8$ RULE. INTEGER FK

DIMENSION A(2) SIMZNI

LOBICAL RE:- $\quad$ SIMJNI

$\begin{array}{ll}\text { LDGICAL RE: } & \text { SIMZNI } \\ \text { PREV } & \text { SIMINI }\end{array}$

C INITIALIZE H, $X_{0} N_{1} M_{1} S$

C.0.0........ $\operatorname{SIM} 3 \mathrm{~N}$

$x=\Delta(1) \quad$ SIMSNI

$N=0 \quad$ SIMZN!

$M=3 \quad \operatorname{SIM} 3 N I$

$S=0 . \quad$ SIMSNI

C LOOP TO COJNT MAXIMUM NUMRER OF EVALUATIONS

C =

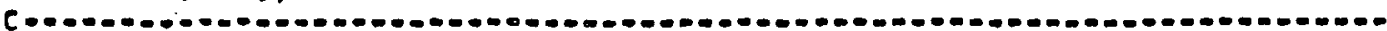

C LOOP TO COJNT TME NIIMUEP OF FUNCTION EVALUATIONS

DIj 1 i=N! $M$

$R=3$. $P$ M

C DETENMINE THE CUEFFICIENT Q

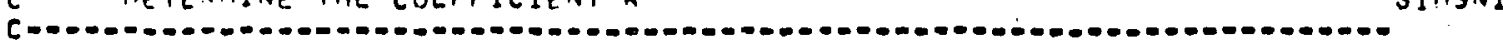

IF (MOD (I, 3) ,EU, 2*N) R=N+1.

C SUIM THE FUMTTION EVALUATIONS

C-D.

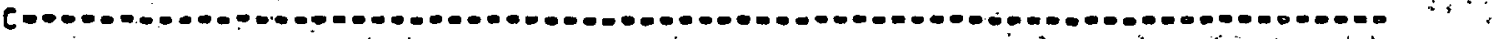

C INCKEMENT X

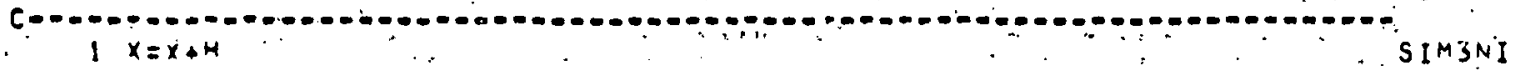

C DUTAIN NEW VALUE OF IITEGKATION

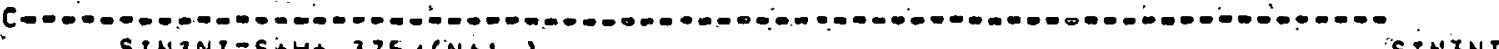
SINZNI $=S: H * 375,(\mathrm{~N}+1$,$) SIMINI$

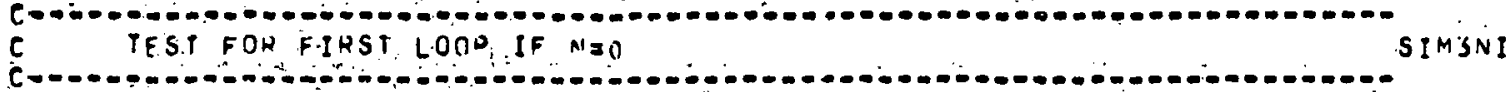

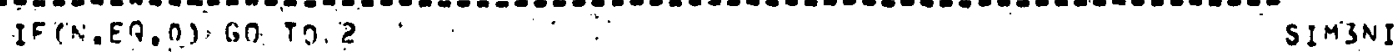

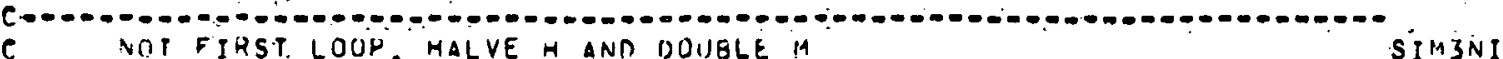
$4=4.5$
SIMUNI $Y=? \times M \quad$ SIMSNI

C. CMECK FOH ERROR CONTROL

C.0.0.-CK FOR ERROR CONTROL 
FUNCTION SI43N!.

$R=S I M I N I$-PREV IF (REL) $R=R / S I M 3 N I . \quad$ SIMSNI

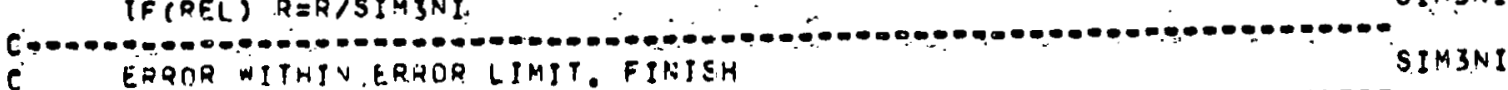

C IF (ARS (R) GL

C SET NEW VALUE OF IMTEGEATION

C-F.0. SE T NEW. VALUE OF INTEGRATION

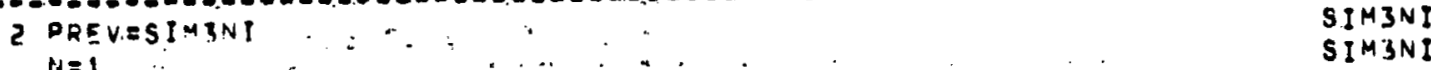

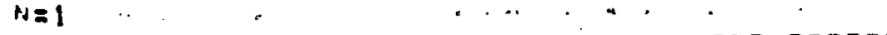

65

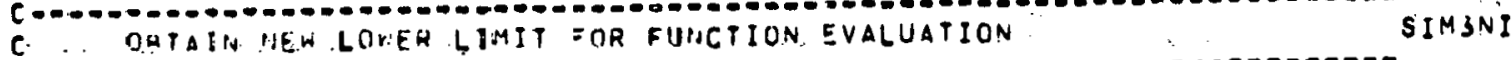
SIMSN?

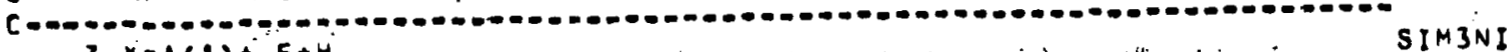

$x=4(1)+.5 * H$

SIMSINI

IER. $=1$.

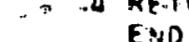


5

20

25
FUPACTION FERMI (ENERGY, I)

COMPLEX R, Z3,Z

AEAL LAN,LAN

COM DIY LAN,LAv, IFOQ8D, EO ALPHA,PI, GAM, ALYAM,RAD, XI

CSINH $(Z)=.5(\therefore)(X X P(Z)-C E X D(-Z))$

$N=F$ HEKGY $.510+1+1$.

$P=S \cap Q T(W: P-1$.

$Y A M=\triangle L Y A B \# W>P$

If $D=0$

CILL GAMMA $(X 1, Y I, T E R)$

$I G O=I E R+1$

60 TO $(10.25 .27) \cdot 160$

$10 \quad 21=Y 1 \cdot+$ ?

$23=C M D L X(Y A M,(1,-G A M)$

$H=P I * 25 / C S I A H(P[\otimes 23)$

$Z Z=C \Delta H S(H)$

IF (IFUHAD.EO.n) GO 105

$C=D+Z+(E Q) E N E R G Y)+2$

riti) $T 0$

$5 C=1$.

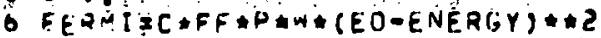

IF $(1, E, ., 1)$ FEHMT =FERMI *ENERGY

The Tineti:

525 pist ant

5T DRTNT HOO

UDO FORMAT, " EHRCR IN GAMMA")

STOP

END: : 
SUAROUTINE GAMMA $(X$, GAMMAX, IER)

C Calcillation of PHE Gamma Funetion DF $x$

DIMENSIDN Q (8)

DATA (A (1),I:1.8)/0.57719:652,.08826.5891,0.897056037,.918200857,

$10.756704078, .482199394 .0 .193527818, .0358683431$

IF(ARS(X).LT.33,) 60 TO :

C THE MAGHITUDE OF $X$ IS GKEA THAN 33

$$
\begin{aligned}
& \text { IERDI } \\
& \text { HETURN }
\end{aligned}
$$

C INITIALIZE FACTER (FACTDQTAL $x$ ) ANO XFACT $(x-1)$

1 FACTOR=1,

$x \in A C A=x-1$.

- If(XFACT,LT.0, G. TO

positive $x$

2 IF (XFACT LT I, 60 TO 5

FACTDREFietuRixFaCt

XFACT XXFACT-1.

$$
60102
$$

C. Negative $x$

3. XFACTEXFACT-1

FACTOR=FACTOR EXFACT

$\therefore$ IF (XF:ACT) 3, T,a

- Ficiorsicifactor

C Caccillation of gamma funetion of xFict 1.

5. GAMMAXEO

Do: $11 \mathrm{i}$ i.

Ixa-1!

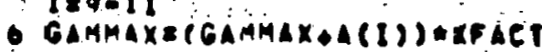

GAMMAXa(1. GAMMAK) OFAETOR

RETURN

T TERT2

AETINAN

END 


\section{FIGURE B-4. Program BIVAR Listing}

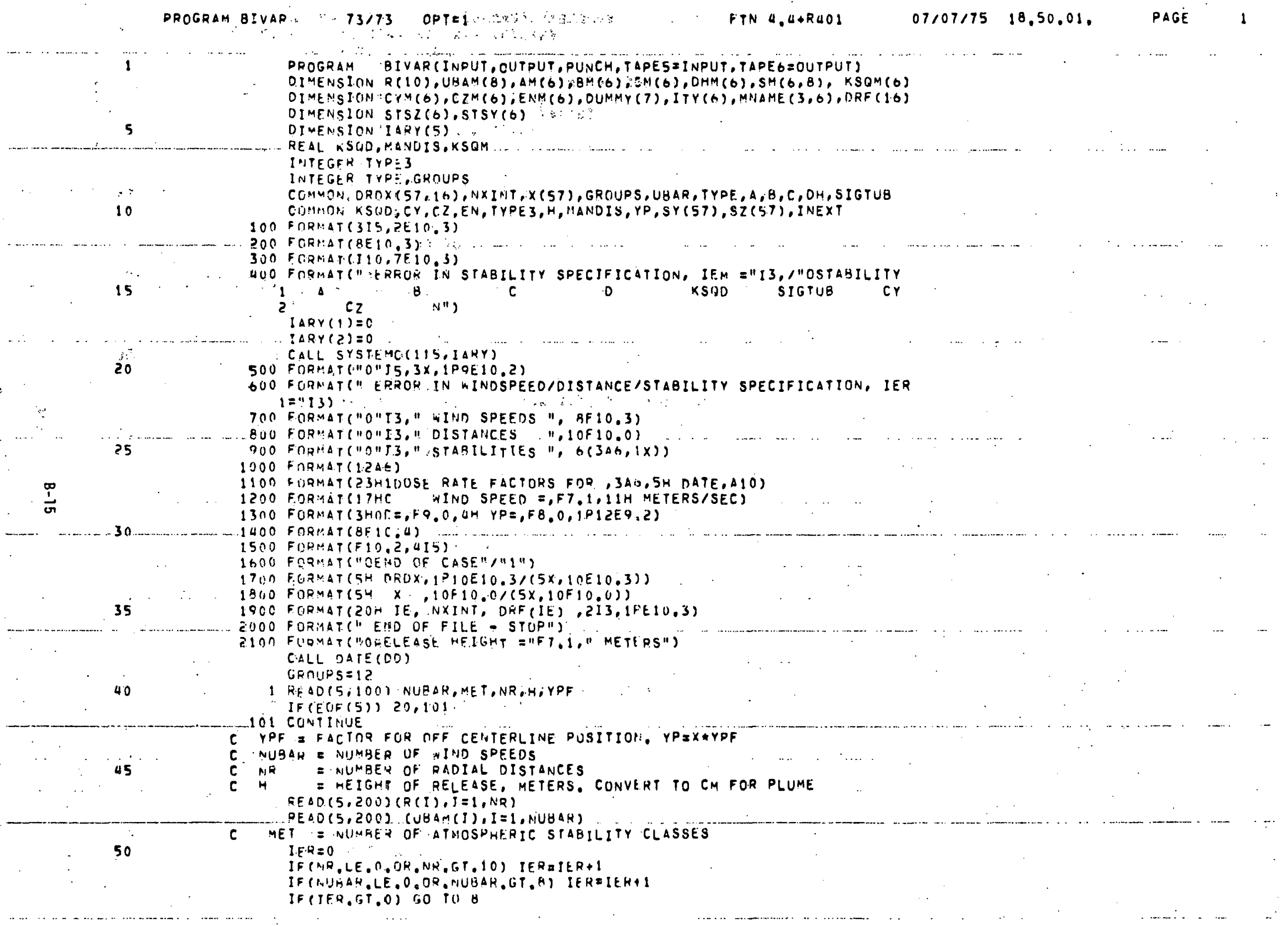


55

DO $2 I=1$, NUBAR

? IF (IJEAM(1),LT,1:E-30I IER =IER+I

U० $3 t=1$, in

3 IF $(P(I), L T, I, E-3 O)$ IFFEIER+1

IF (MET.GT,G,OR:MFT LE. O) IERZIER+

IF (YOF.GT OS) -IEREIEH +I

IE(IER,GT,O) GO TU Y

HHEH

$A=100.44$

C READ ATMOSPHEDIC STABRLITY DATA

READ. $100 \cap,($ (MNAYE $(I, J), I=1,3), J=1$, ME.T)

IEMED

ON O. IMI $1, M E T$

READ $\left(5,300^{\circ}\right)$

ITY (IM), DUMMY

TTYP=ITY(TM)

IF (IPYP.LT.I) IEMEIEM+I

IF (ITYD.GT.9) IEM $=$ IEM+I

70

IF (ITYP.G.T.3) GD TO1 6

JE (ITYP.EO.Z) COO TO 5

IF (IPYP.EA.I) GO PC a

75 IF(ITYO,EQ.3) GO TC LOS

IEM $=I E M+I$

50 TO 6

$4 \triangle M(I M)=\operatorname{DUMMY}(1)$

$I M(I M)=D U M M Y(2)$

$\frac{1}{\sigma} \quad 00$
$\operatorname{DM}(I M)=\operatorname{DUMMY}(3)$

(FPIYYP,ER,1) READ 200, (SM(IM,I),I=1,NLBAR)

KSOM $(I M)=$ DUMAY $(5)$

60 TO

A5

5 CYM(IM) 2 DUMMr (1)

CZM(IM) $\approx$ DUMMY $(2)$

ENMM (IM) = OUMMY (3)

- go to 6

108 STSYCIM) EOUMMY (1.)

STSZ $(I M)=$ DUMMY (Z)

- CON.TINUE.

JF (IEM,EO.O) GD TO

FRINT 400 . IEM

CO 7 IME 1, MET

95.

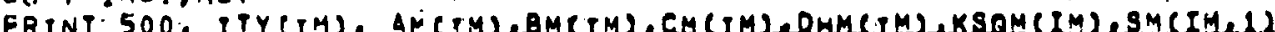

1. $\operatorname{CYM}\left(I_{M}\right), \operatorname{CZM(IM),EMM(IM)}$

CONTINUE

STOP

3F(IER.GT.0) PRINT GOO.IEM

PRINT 700, NUBAR, (UEAMCI), I II,A)

PRINT. B00, NR, (R(I) I I I. I I $)$

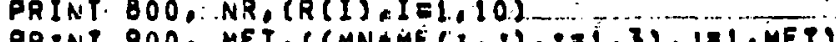

PRINT 900, MET, ( (MNAME $(1, J), 1=1,3), J=1$, MEP

DRINT 2100,4

I.F (IER,GT,O) STOP

105

PIINCH 1500, HH, MET, NUBAR, NR, GNOUPS 


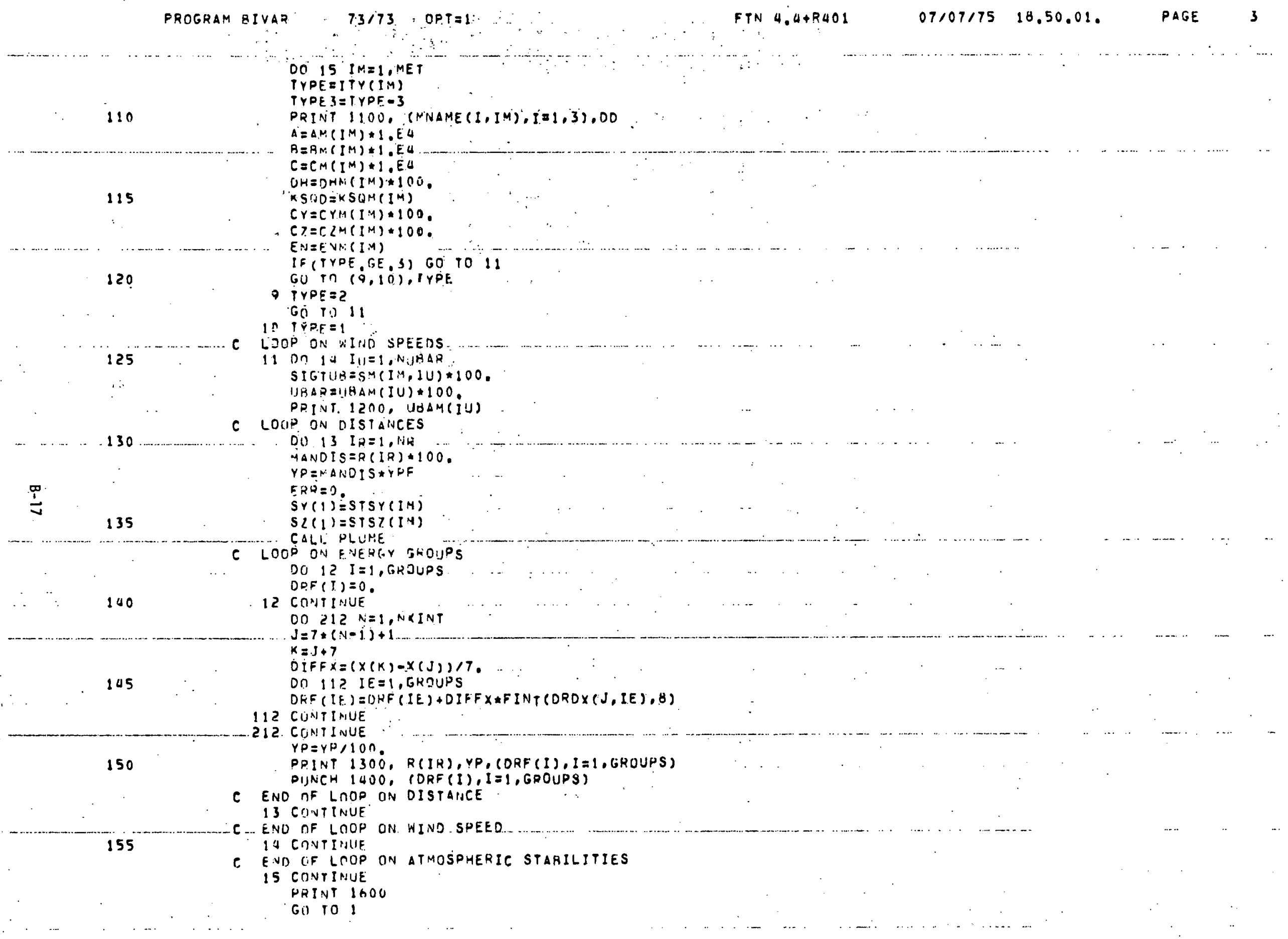


DIMENSION T(57),ZLIM(2Z),YLIM(?Z), EOIERQ(57)

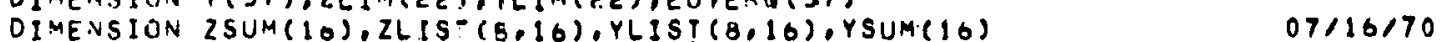

OTMENSION" DEP(57)

DIMENSION Y (ZZ), Z (Z2), YPM(I0), ZPM(10)

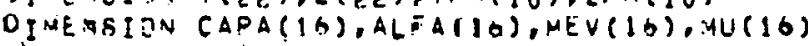

INTETER TYPE,TYPEZ,GRDUPS, VERT, DOSIT

15

INTESGER ENERGY

PEAL MU, MEV,KSUO, MANOIS

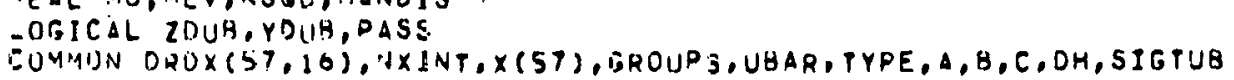

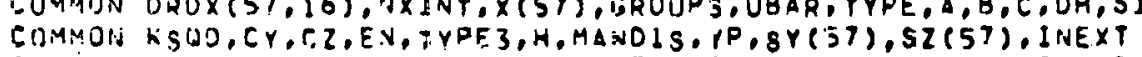

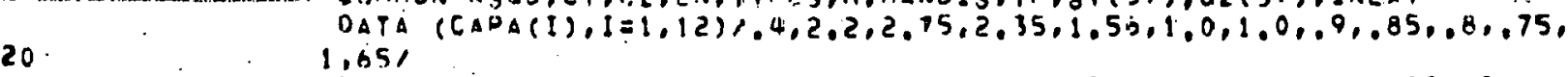

DATA (ALFA(I), $I=1,12) / .0006, .143, .754 .1 .287,1.287 .0831, .402, .211$,

$1.0937, .0373, .0204,01031$

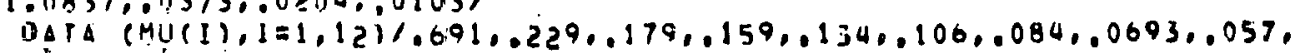

25

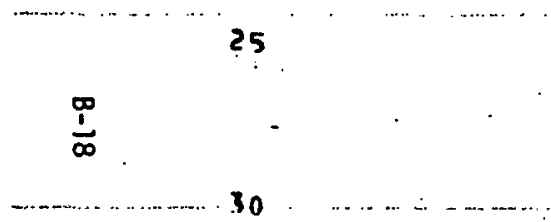

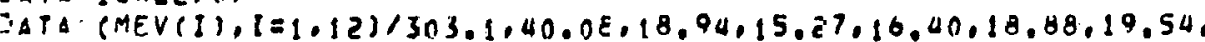

18.93,17.58,10.16.15,04.13.191

IFCICALL.GT:OU 60 TU 3

\section{GNOUPS $=12$}

DO $21=1,12$

2. MU(i) $\mathrm{MUU}(1)+1.293 E-5$

3 ICALLEICALL+1

PASS $=$ FAISE.

MYP $\triangle S D R T(H: H+Y P * Y P)$

$08 / 19 / 70$

35

DMAX $=50000$ Q

USTHR=15000. 2 , \#HYP

UP TWOE $5000,+Z, * H Y P$

UPONE $=1500$

40

C UPDIS IS UDWIRD INTEGRATICN DISTANCE.

UPDIS =AMINI (BOOUO, MANOIS, UPMAX)

C. OWND:S IS DONNNINO INTEGKATION DISTANCE... (MAY BE.NEGATIVE)

DUNDISIANINI (BOOOO , UPMAX)

C TEST : UPWIYD PLUS DUWNWINO DISTANCES ZERO IMPLIES NO EXPOSURE

IF ( IPOIS+UNNDIS) $13,13,15$

13 PDINT IH. UPDIS.DWNUIS

IL FRMMATC" NO X-INTEGRATION INTEAVEL, UPOIS ERIPEIO.Z," METERS, DOWN $1015=" E 10.2, "$ "METERS")

$$
\text { SiOP }
$$

C TEST - IF DNADIS LESS THAY IJOCM, SKIP ORWNWINE INTEGRATIUN. IS IF (DNNDIS.LE.100,) GIS TJ IS

C TEST- IF MAMIIS LESS IMAN 100 CM. SKIP UAWIND INTEGRATION. it (MSNOIS.LT,IOO,) 60 PD 2 ?

$08 / 19770$ 
55

C SET UPRITNO. INTEGRATION

$x(1)=M A N D I S-U P D I S+10$.

IF (IIPTHR.GT.JPOIS-3000,) GO TO 16

$X(B)=M A N D ! S-U P T H R$

$x(15)=1 A N D$ IS - IJPYWO

$X(2 ?) \equiv M A N D I S-U P . B N E$

60

$x(29)=M A N O I S$

NXINI $=4$,

$$
\text { Gก TO IA }
$$

16 IF (UPTAO.GT.UPDIS-1000,) GO 10 17

$x(A)=N \Delta N$ IS - UPT:AO

$65 \quad \ldots \quad \ldots \quad \ldots(15)=M A N D T S-U P O N E$

$x(2 ?)=M G N D I S$

$M X I N T=3$

Go To 18

17 IF (UPONNE, R:T UPOIS-300,; GO TO 1017

70

$x(B)=M A N I S-U D O N E$

$x(15)=M A N D I S$

NXI.NT = ?

Gก. $110 \cdot 18$

$1017 \times(8)=$ MANOIS

$$
N \times I N T=1
$$

18 [NEXXTET: $(N \times[N T+1]+1$

SET UDARIWINIS INTEGRATIUN

$x(T N E X T)=P \triangle N D I S+U P O N E$

$x(I: V E X T+7)=H A$ HISIS +UPTWO

C $80 \quad X(I: I E X T+14)=$ MANDIS +UPTHR

क $\quad x($ INEXT+2I) $=M A N D I S+D W N O I S$

IAYTNT $=N \times I N T+M$

GO 1035

$51 \times(1)=M A N D I S$

$85 \quad \begin{aligned} & \text { NXINT }=0 \\ & \text { GO TOI IH } \\ & 25 \text { CINTINUE. }\end{aligned}$

$85 \quad \begin{aligned} & \text { NXIHT }=0 \\ & \text { GO TOI IA } \\ & 25 \text { CONTINUE. }\end{aligned}$

C CALCULATE X-GRID POSITIONS
31 CUINTINUE

Uil $33 \quad 1=1, N \times I N$

$J=7 *(1-1) \cdot 1$

$x=3+7$

[If $x=(x(x)-x(j)) / 7$.

Ui. $32 L=2,7$

PCSI $1=7 *(1-1)+$

$x(D I S I T)=x(P O S I T-1)+D I F F X$

32 CONTINUE

33 CONTIHUE

C CALCHIATE TPAVEL TIME TO EACH POSITION

IA.EXT=7*H]X.?NT+1

DO $34^{\circ} I=1$, INEXI

$T(1)=x(I) / U B A R$

UEP $(I)=1.0$

34 CONTINUE

105

C CALCIIIATE SITMA AND SIGMA 2 FOR EACH IOSITION

39. IF (T.YHE.LE. 3) 6O TO U5
$08 / 04 / 70$

$09 / 15 / 70$ 


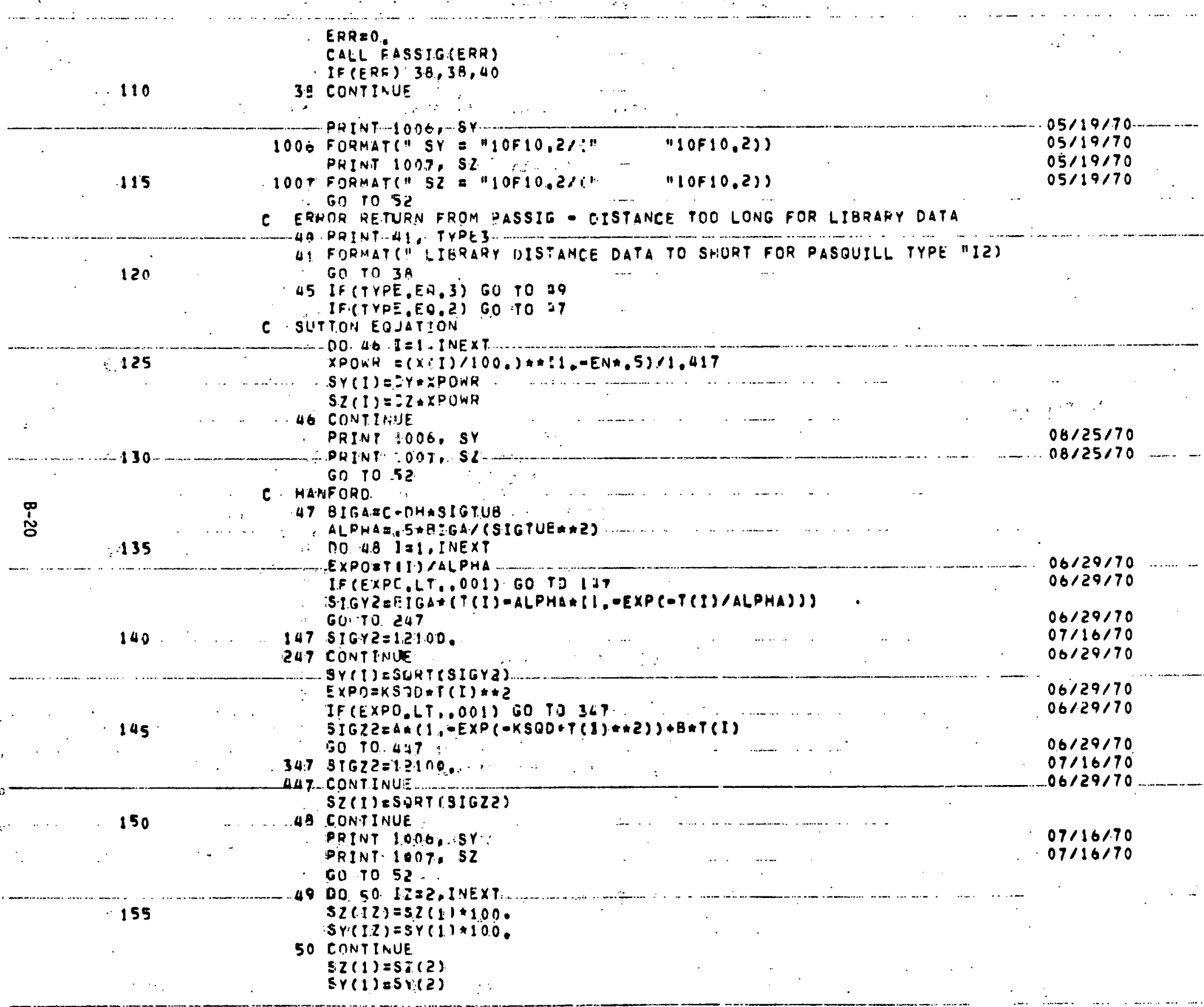




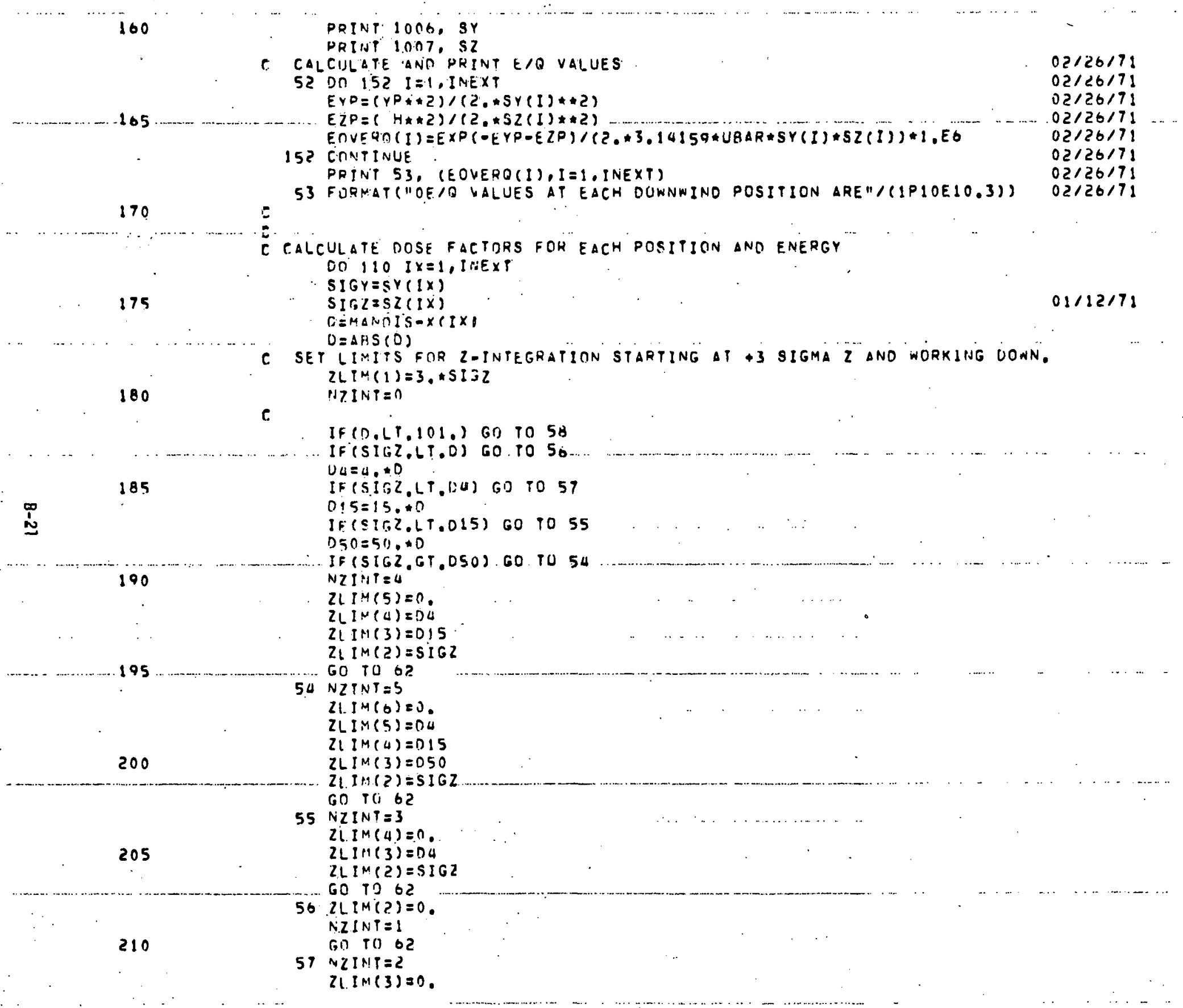




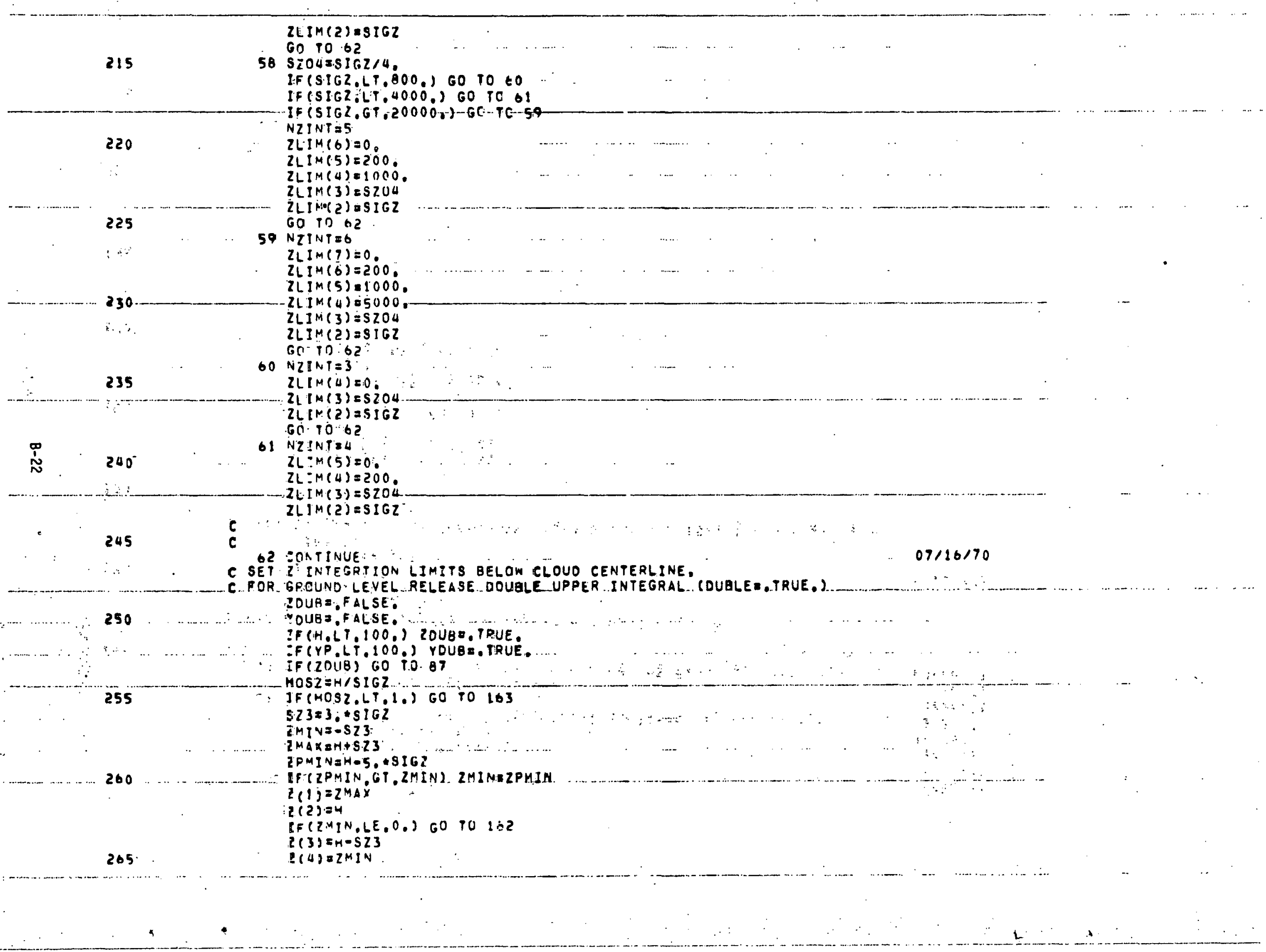


NZINT $=3$

1162 NISNZINTTII

OD $1202 \quad I=1, N$

270

1262 ZL.IM $(1)=Z(I)-H$

r., $10 \quad 87$

$1622002=412$.

$2 N 10=523$

IF $(\angle 62)$ LE.ZMIO) ZMID=Z(2)

275

IF $(Z P S) . L E \cdot Z M I D)$
IF

on 26 ? $j=i, n z I N T$

262 ZPM(J) $=$ ZLIN(NZINT-J+I)

Dij $362 \mathrm{~J}=1, \mathrm{NZ}: \mathrm{NT}$

,

IF(ZDM(J).GT.ZMID*.9) GO TO 462

280

362 CUNTIIUE

$N Z=N 2 I N T$

Gn TO 562

$105 \quad N Z=7-1$

502 IF (NLEEQ.0) 6.3 TO 762

285

OO $602 \mathrm{~S}=1, \mathrm{NZ}$

$662 \cdot 2(\mathrm{~J}+2)=7 P M(N Z-J+1)$

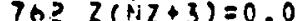

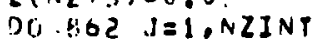

$i=N Z+J+3$

290

$Z(I)=-2 L 1 M(N Z I N T+1-J)$

IF (Z(I),LT.ZMIN) 60 TO 962

862 CONTINUE

$\begin{aligned} & \tilde{D} \\ & \tilde{\omega}\end{aligned} \quad 962 Z(1)=2 M I N$

295

1002 NIINTSI-1

G.j. 101162

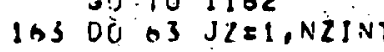
$Z Z=2 * Z L I M(N Z I N T-3 Z+1)$

IF(ZZ,GT,H) GO TO GU

300

3 CUITINUE

$j z=j l+i$

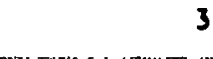

$6,4 \quad K Z=j z-1$

$i z=z+j z+1$

INDEX $X=L Z+N Z I$ INT

IF (JZ.GT.NZINT) INOEX = INDEX-I

305

IF (KL,L.T.1):60 TO.66

DO $65, I=i, k z$

ZLIHI VZINTTI+I) =-ZLIM(NZINT-I+1)

ZL.IM (INUEX-I) =-H+ZLIM(NZINI-I+I)

310

OS CONTINUE

So ZLIM(INDEX) $=-H$

IF (JZ.LEONZIVT) ZLIMPNZINT +JZ +1) E-HIZ,

DO $67 . I=1, N Z I N T$

ZLIM (INDEX $+I)=-H-Z \operatorname{LIM}(N 2 \operatorname{INT}-I+1)$

b) CONTINUE

MIINI I INDEX +NZINT-I

315

BT CUNTINUE

C SET Y INTEGRATION LIMITS STARTING AT O. AND WORKING UP. SIGYZ=SIGYASIGY

$07116 / 70$

$07 / 16 / 70$

$07116 / 70$

$07 / 16 / 70$

$07 / 16 / 70$

$07 / 16 / 70$

07116170

$07 / 16 / 70$

$07 / 16 / 70$

$07 / 16 / 70$

0716770

$07 / 16 / 70$

$07 / 16 / 70$

$07 / 16170$

$07 / 16 / 70$

$07 / 16 / 70$

$07 / 16 / 70$

$07 / 16 / 70$

07116170 
320

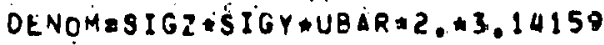

SIGZ2=SIGZ+SIGZ

SY 3 a3. $\$$ SIGY

DSIGY $=S I G Y$ ISIGY*2,

$325 \quad$ ZSUM(ENERGY) $=0,0$

9. CONTINLE

Dก $108 \quad I Z=!, N Z I N T$

DELZ $=(Z L I N(I Z)-Z L I M(1 Z+1)) \cdot 9$.

$Z 2=71$. IM(IZI+H.

$330 \ldots \ldots$ DC. 106 VEE $1 \equiv 1$; 8

$Z M-2=(2 Z+F)+M 2$

VERTEXะ.5*2MHZ/51622

........... 330

PERPZEO\#D+ZZ\#ZZ

335

PERPISURT (PEKNZZ)

$Y L[M(1)=0 ; n$

IF (PERP. LT.10:0) 60 T5 93

IF (SIGY.LT.PERD) 60 TS 91

IFISIGY. DERP

$\quad 34$

IF́(SIGY:LTPERPA) 601092

PERP $15=15$ \&PERP

IF (SIGY IT PERP 15) GO TO

PERPSO 50 , DERP

$07 / 16 / 70$

IFCSIGY.GT.PEKPSO) GO TO 189

$07 / 16 / 70$

唸

NYIINT $=4$

YLIM $(2)=P E R P S$

YLIM (3) $=$ PERP I 5

.......................

350

$Y L I M(5)=5$ Y

50 To. 9.6

I89 NYINT $=5$

YIIM(3) =PERP 15

LIM( 5$)$ ESIGY

$07116 / 70$

$07 / 16 / 70$

355

$P(I M(6)=S Y 3$.

$07 / 16 / 70$

60 T0 96 :

$07 / 16 / 70$

O RYINT $=3$

YLI $Y(2)$ IPEAPA

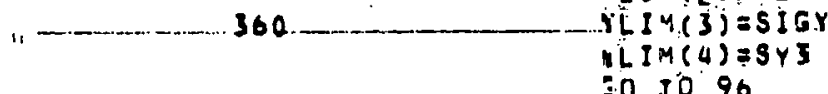

$360 \quad r L I Y(3)=5 I G Y$
$Y I M(4)=S Y I$

01096

a) $\operatorname{LIM}(2)=5 Y 3$

365

i $Y I N T=1$

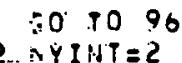

HLIM (.Z) $=S I G Y$

$P L I M(3)=S Y 3$

aी 1096

370

.03 sroues $[G, Y / 4$.

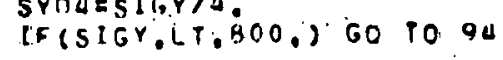


IF(SIGY.LT.4000.) SO TO 95

$07116 / 70$

ISTSIGY.GT.20000.) GO TO 193

$07 / 16 / 70$

3.75

NYINT $=5$

$Y \operatorname{LIM}(2)=200$

$Y \operatorname{IIN}(3)=1000$

$Y \operatorname{TIH}(4)=S Y(14$

$Y L I M(5)=S I G Y$

$Y L I+1(6)=S Y 3$

.380

Go ro.

[9] NYINP $=0$

YLIM $(2)=200$.

$Y L T M(3)=1000$.

07116170

$Y(I M(\Delta)=5 ; 000$.

$07 / 16 / 70$

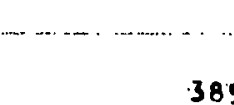

385

$Y \operatorname{LIM}(5)=5 Y 04$

$07116 / 70$

$Y L I M(6)=S I G . Y$

07116170

Y $I M(6)=S I G$

$07 / 16 / 70$

G) 10.96 .

$07 / 16 / 70$

G) 10.96

$07 / 16 / 70$

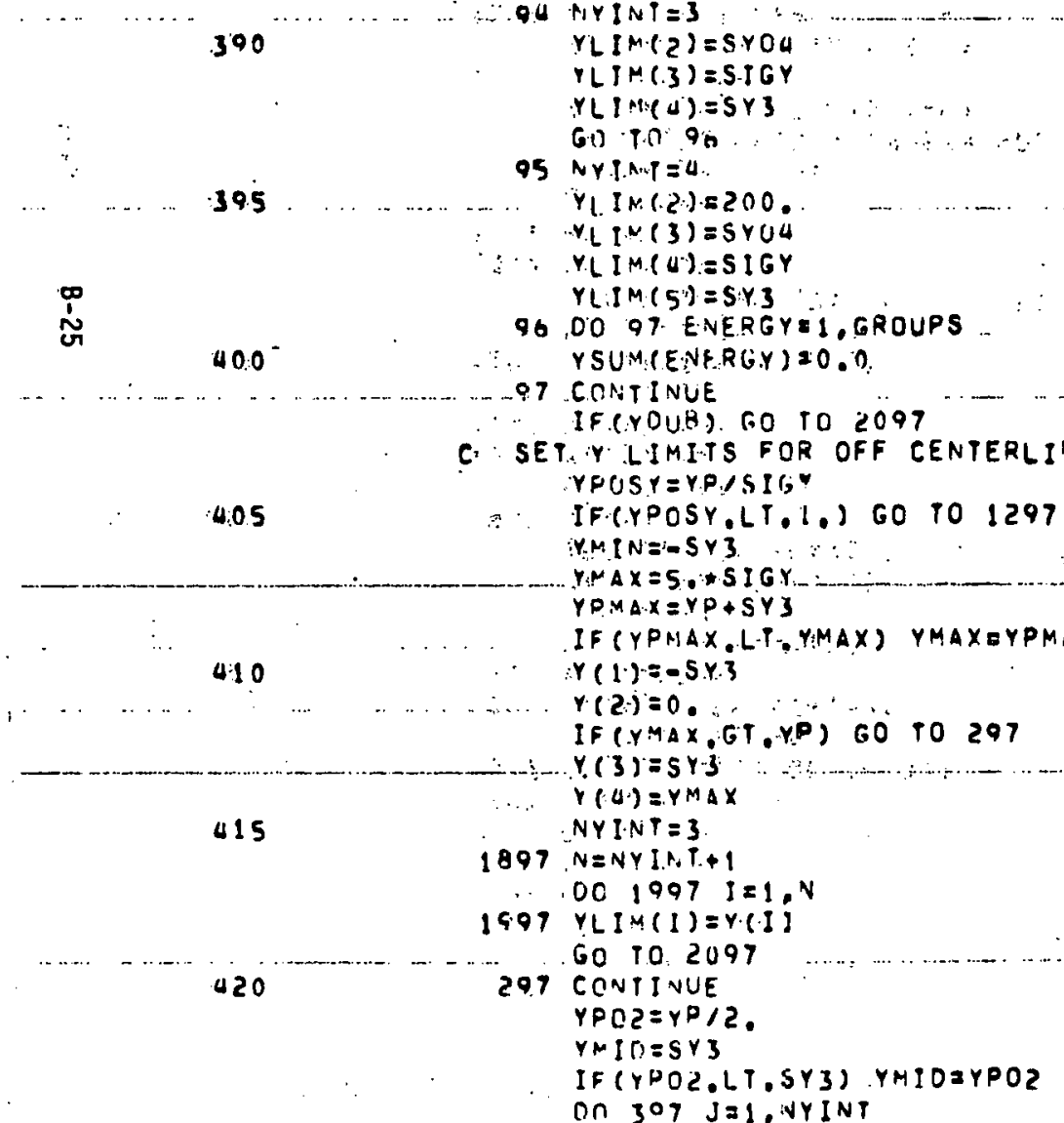


SUBROUTINE PLUME : : $731.73 \because$ OPI=1: $\ldots$

425

39.7 YFM.(J) $=Y P-Y(I M(J+1)$

DC 497 J8I.NYINT

IF(YDM(J),LT, YMID) GO TO 597

49.7 CONTINUE

NYYNYINT

430

Q

:0.) 60, ro 897 .

On .777: $\mathrm{J}=1, \mathrm{Nr}$

$709 Y()+2.)=Y P ! M(P, Y+1-. j)$

$807 Y(V Y+3)=Y D$

DO $007 \mathrm{~J}=1$, NYINT:

$I=\sqrt{ } r+3+J$

$r(I)=Y P+Y(\operatorname{IM}(J+1)$

IFP(I) GT, YMaX) GO TO 1097

440

997 CONTINUE

GO

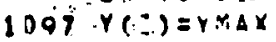

1197 NY $=$ : T. $=1-1$

$445 \quad$ 129.7. DO 1.397. NYEI,NYINT

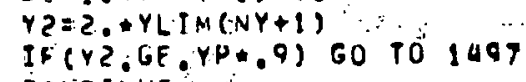

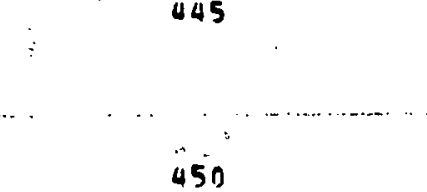

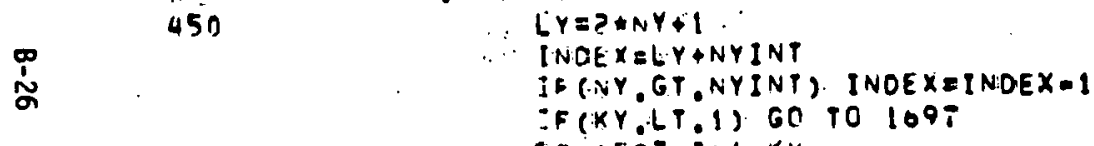

$455 \quad Y(N Y I N T+I+I)=Y L I M(I+1)$

1597. Y (INÖEX-I) $=Y D-Y L I M(1+1)$

1697 (.INDEXXIZYP.

[F (NY.LE.NYINT) Y(NYINT+1+MY) aYP/Z.

CO 1797 I I I.,NYINT

.460

$Y(I N D E X+I)=Y P+Y L I M E I+1.1$

1797 (NYINTOI+I).Z=YLIM(I+I)

P. (NYINT+1.)=0:

MYINTENYINTIINOEXOI

$G D$ TO 1897

465 2097 VEXP.OEEXP(-VEFTEX)

CODOLOOP ON NUMBER. OF

OS IOU IYY I, NYINT

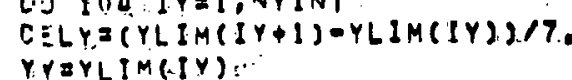

470 C DO LOOP.ON Y POSITIONS WIIHIN MAJOR INTERYAL

DO 102. LATII,8.

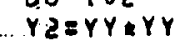

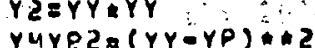

RSA

C CALCULATE INVERSE SQUARE FACTOR FOR CURAENT $X, Y, Z$ POSITION:

475

EOD=EXP(-YZ/OSIGY) IOENEM 
IF (R.LT.I) GO TO 90

GNUM $=1$.

IF (R.GT,140,) GO TO 98

GNUM $=1 .-E \times P(-F S O * .0006283196)$

QR GRATIO= GNUM/ (12.56630*RSQ)

$$
\text { GO TO } 100
$$

485

\section{Q0 GRATIRZ.00005}

C CLLCULLTE JOSE RATE KERNEL FOR EACH ENERGY GROUP AT $X, Y, Z$.

10O VGEVEXOOGHATIOLEOB

OPI 101 ENERGY $=1$, GROUPS

HMII $=$ MUU (ENERGY) $R$

$11 / 18 / 70$

ATTEN=EXP(-RMJ)

$11 / 18 / 70$

490

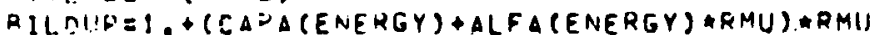

YLIST (LAT, ENERGY) =VG ATTENABILDUP MEV (ENERGY)

101 CIIVTINUE

$r Y=Y r+O E L Y$

495

10.5. CRNIINUE

DO 1 OI ENERGY 1 , GROUPS

IF (YOUB) GO TO 1103

YSUM'(ENEHGY) EYSUM (ENERGY) +OELY \#FINT (YLIST (1, ENERGY), 8)

Gr: 10.103

500

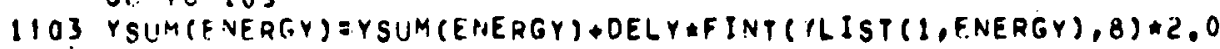

ICI CONTI:UE

ICA CONTINUE

DO 105 ENERGY $=1$, GROUPS

ZLIST (VERT, ENERGY) =YSUM (ENERGY)

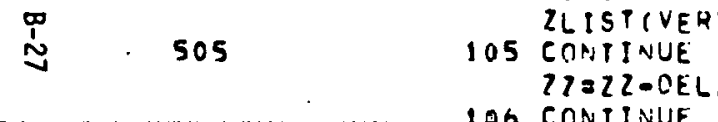

C INTEGRATE ANO SUM OVER $Z$ FOR CURRENT X PCSITION

DO IOR ENERGY $=1$, GROUPS

IF.(ZOUE) GO TO 107

2SU?: (ENERGY) = ZSUM (ENERGY) +DELZ Z INT (ZLIST (1,ENERGY), 8)

GU TO 108

1.07 . ZSUN (ENEKGY) $=Z$ SUM (ENERGY) \&ELZ*EINT (ZLIST $(1, E N E R G Y), 8) * Z$

IOB CONTIPUE

515. DC 100 ENERGY = 1.GROUPS

DRDX (IX, ENEERGY) = ZSUM(ENERGY)

ZSUM(ENERGY) $=0.0$

109 CONTITIUE

$520-110$ CONTIAUE

$05 / 19 / 70$ 


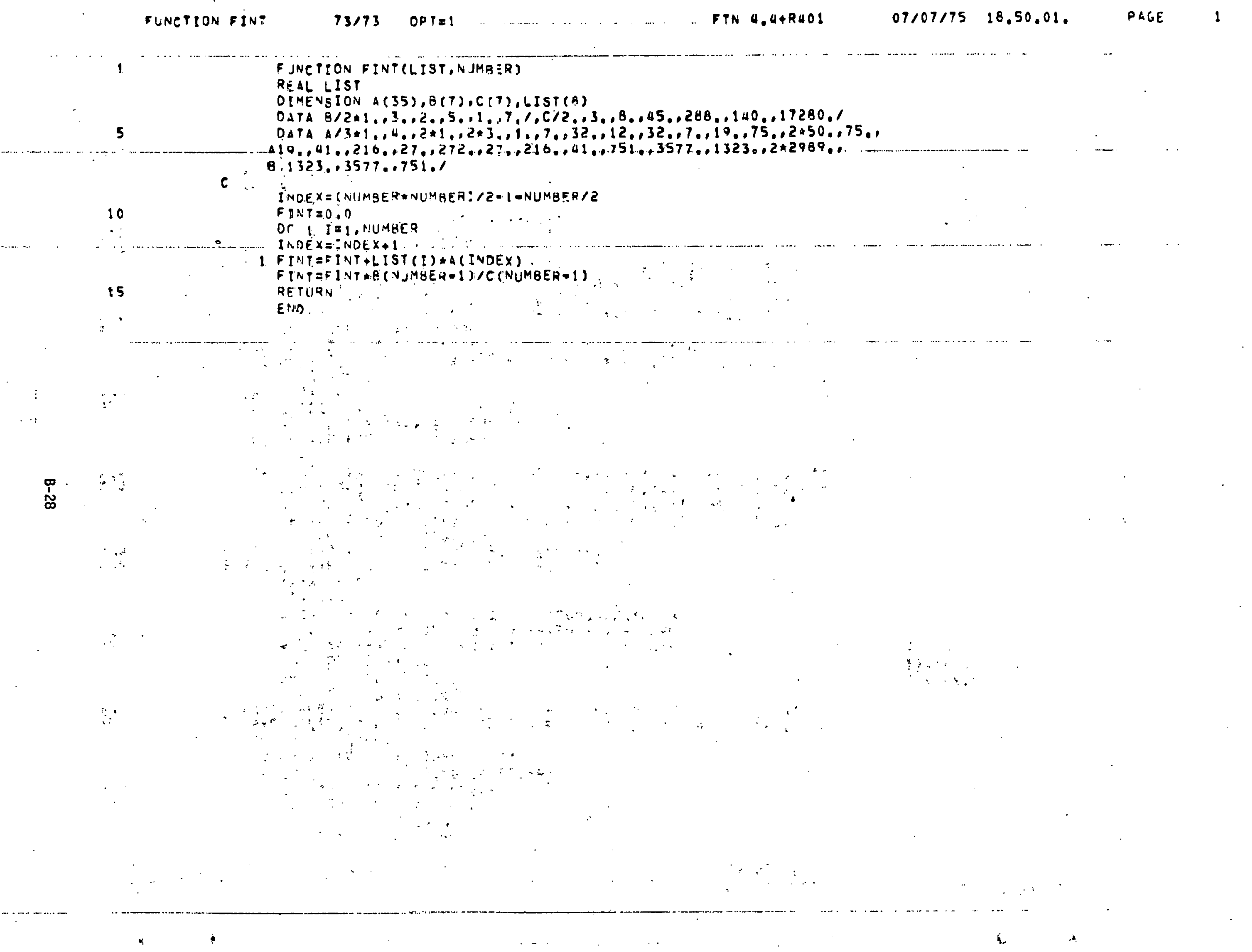


SURROUTINE PASSIG(ERR)

INTEGFA PYPE, TYPE. B, GHOIJPS

I.VTE GFA PYPE, TYPE, 3, GROIJPS

CDMMUN DRDX (57,16), NXINT, X(S7), GROUPS, UHAR, TYPE, A,B, C.DH, SIGTUB

COM,YON KSWD,CY,CZ,EN, TYPEZ,H, MANOIS, YP,SY $(57), S Z(57)$, INEXT

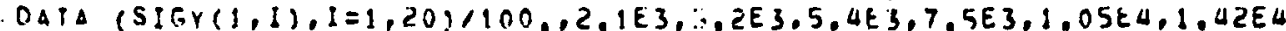

$1,2, E 4,2,9 E 4,4.5 E 4,6,1 E 4,3,3 E 4,1,12 E\}, 1,55 E 5,2,2 E 5,3,4 E 5,4.5 E 5$, $26.255,4.25 .5,1.1561$

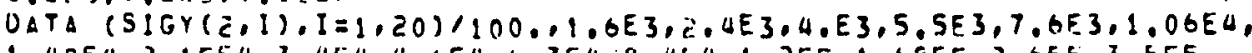

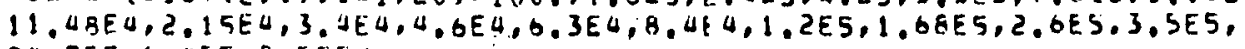
$24,7 E 5.6$, HE. 5.8 . SES

OATA (SIGY $(3,1), 1=1,20) / 100,1,2 E 3,1,75 E 3,2,85 E 3,4, E 3,5,5 E 3,7,6 E 3$, $11.05 \% 4,1,55 E \mathrm{H}, 2.4 E 4,3.3 E 4,4.5 E 4,6,16,4,8,5 E 4,1,2 E 5,1,85 E 5,2,5 E 5$, $23,4 E 5,4,7 E 5,6,3 E 5.1$

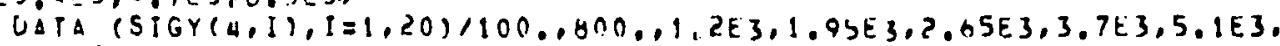
$17.253,1.04 E 4,1.5 E 4,2.2564,3,1 E 4,4,2 E 4,5,7 E 4,7.1 E 4,1.25 E 5,1,7 E S$, $22.3 E 5,3,55,4.1 E 5 /$

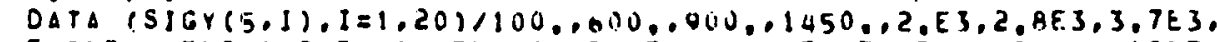

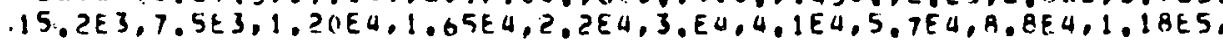
2!., HE 5, 2.1F5.2.8E5/

DATA (SICY $(0,1), 1=1,20) 1100,0390 \ldots 6(0, .980,1350,1850, .2550,0$

$13000,55200, \ldots 8100, .1,1 E 4,1,53 E 4,2,1 E 6,2 ., 8 E 4,4, E 4,6,1 E 4,8,264,1.12 E 5$ 2.1., URES, 2.ES\%

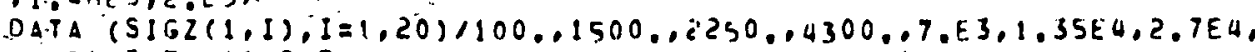
$16.7 .64, ? . E 5,11 *$ ?.E5

DA.TA (SIGZ(2,I),I $=1,20) / 100, .1, E 3,1500,12550, .3700,05700, .8600 \ldots$

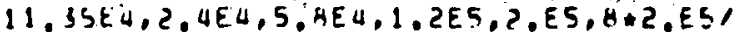

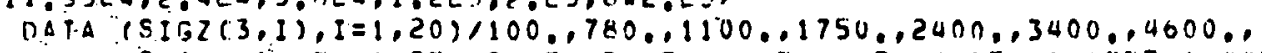
$16400 ., 9000,1.0 E 4,1.9 E 4,2.6 E 4,3,4 E 4,4.4 E 4,6, E 4,8,9 E 4,1,12 E 5,1.44 E 5$ $2,1,78 E 5,2, E S /$

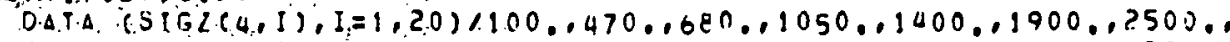
$13300 . .4300, .6200, .7500 . .9500 \ldots 1$. 1SE4.1.4E4.1.7E4.2.2E4.2.05E4 $23,2.54,3,750,4.5 F_{-4}$

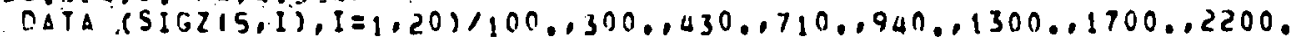

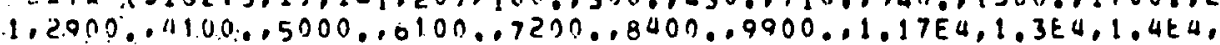
2. $1.550 .1 .7 E 4$

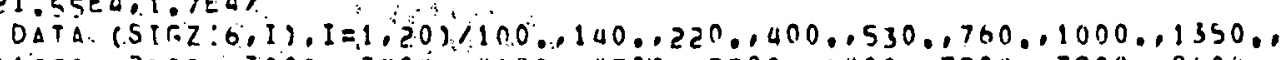
$11770 \ldots 2500,03000 \ldots 3500, .4100 \ldots 4700 \ldots 5500 \ldots 6400 \ldots 7200 \ldots 7000 \ldots 8000 \ldots$ 20400.1

DATA (OIST(1), I $=1,20.) 1.0,1,1, E 4,1,5 E 4,2,5 E 4,3,5 E 4,5, E 4,7, E 4,1, E 5$,

$11.5 E 5.2 .5 E 5,3.5 E 5,5 . E, 5.7, E 5,1 . E, 0,1,5 E 6,2.5 E 6,3,5 E 6,5, E 6,7, E 6,1 . E 71$

C. CALCULATE SY ANO SZ FOR EACH X POSITION DESIRED

IOATA =

$E R R=0$.

DC $19 I=1, I N E X T$

1 IF. IDATA.GT.ZO) GO TO A

2 IF (SIGZ(TYIPEZ, IOATA)) $B, 8,3$

3.IF $(X(I)-D I S T(I D A T A)) \quad 6,5,4$

U IDATA $=1 D A T A+1$

GO TO 1

$5 S Z(I)=S I G Z$ (TYPEZ.IDATA)

gO 100

- If (InATa.t.o.1) go to 5 


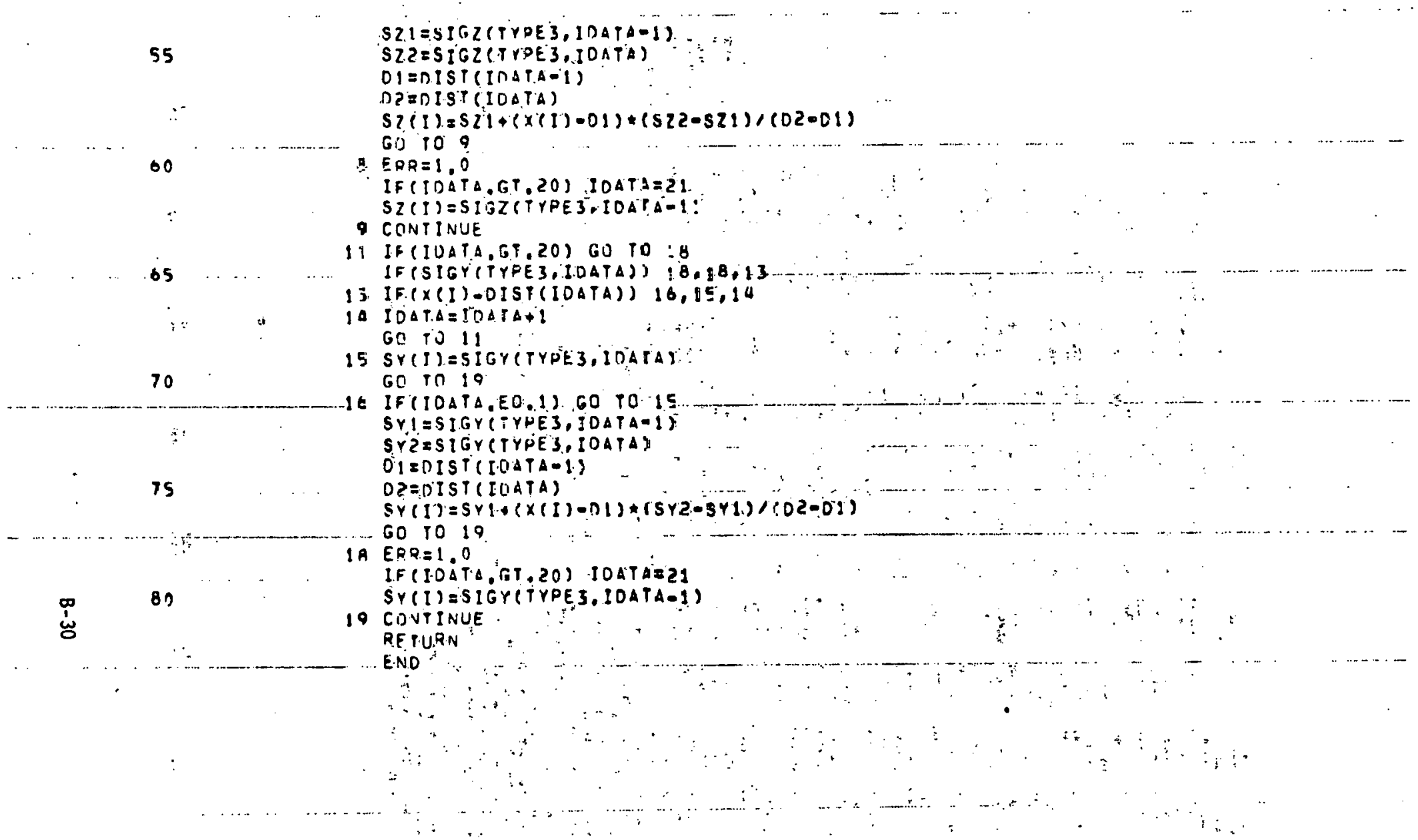

$S 2.1=S I G Z$ (TYPEZ, IDATA-I)

IF (IDATAGT.2O) IOATA=21

I.F (IUATA GT. 20 ) GO TO:B

3. IF.(X(I)-DIST(IDATA)) 16,1E,14 


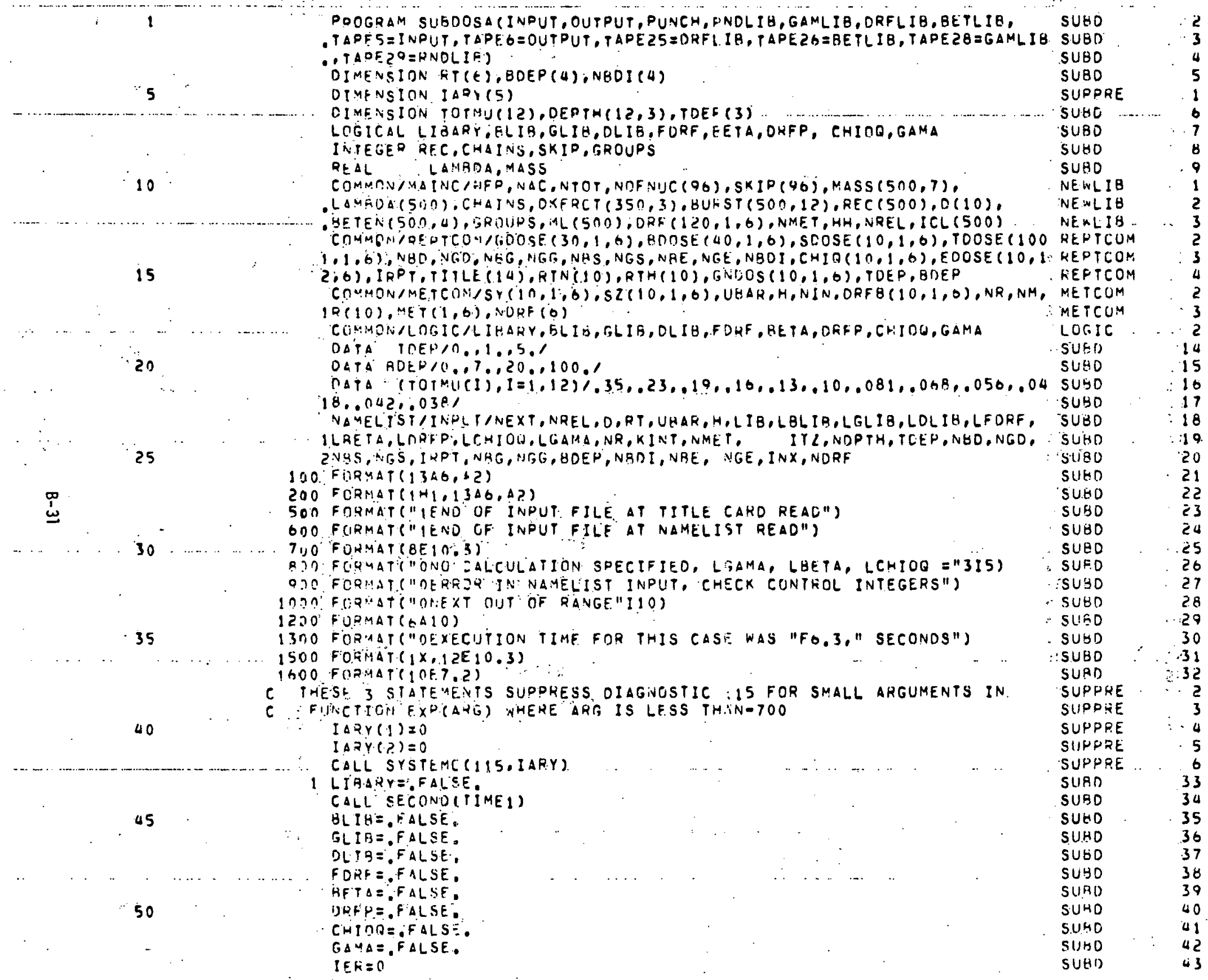




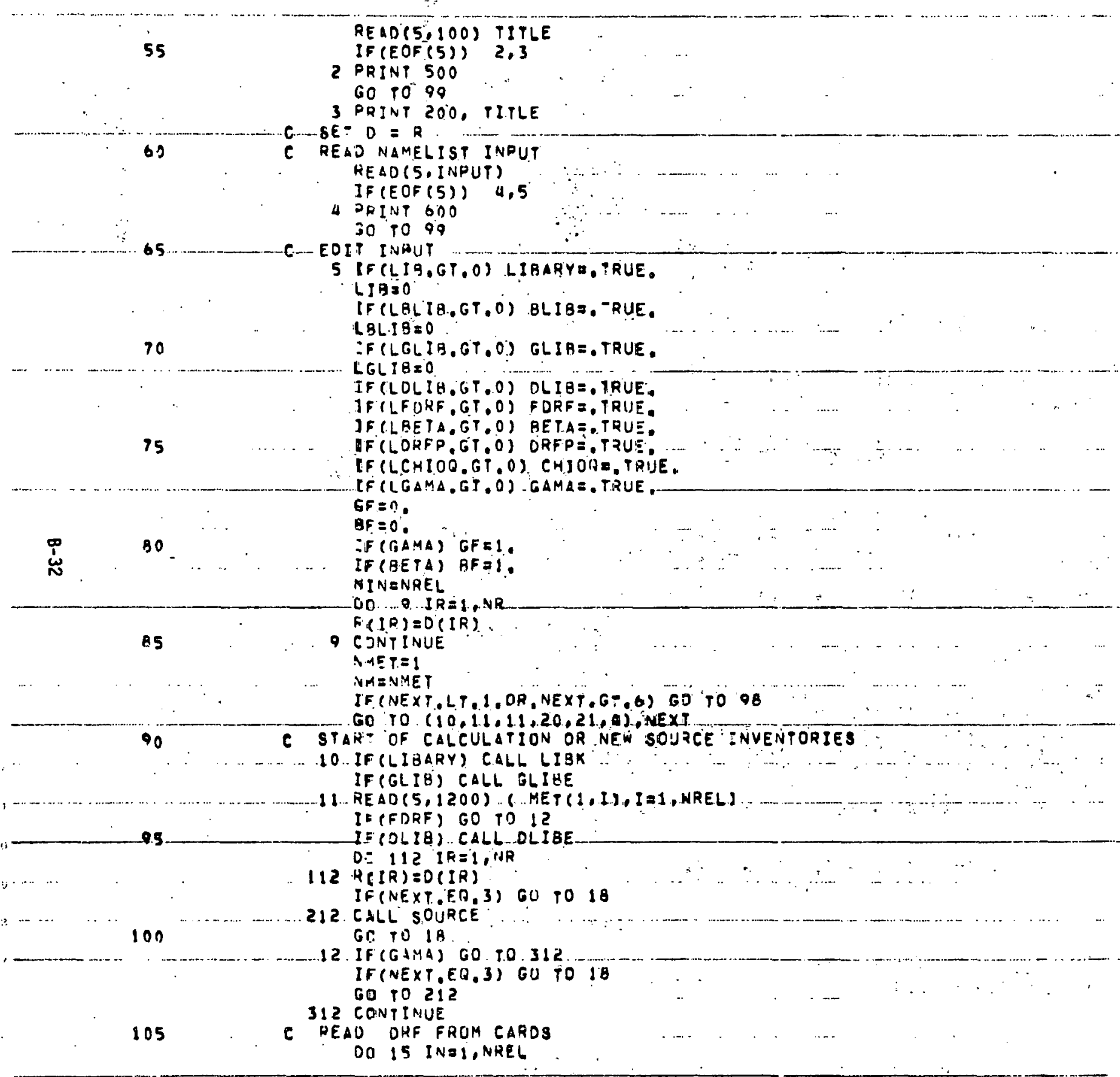

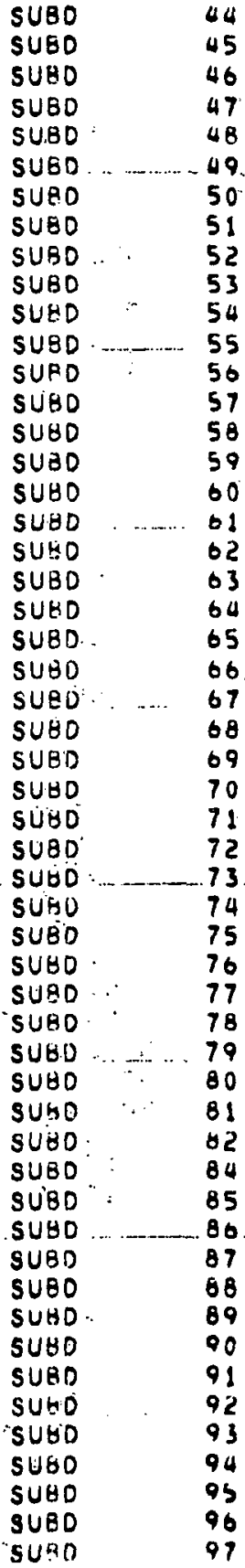




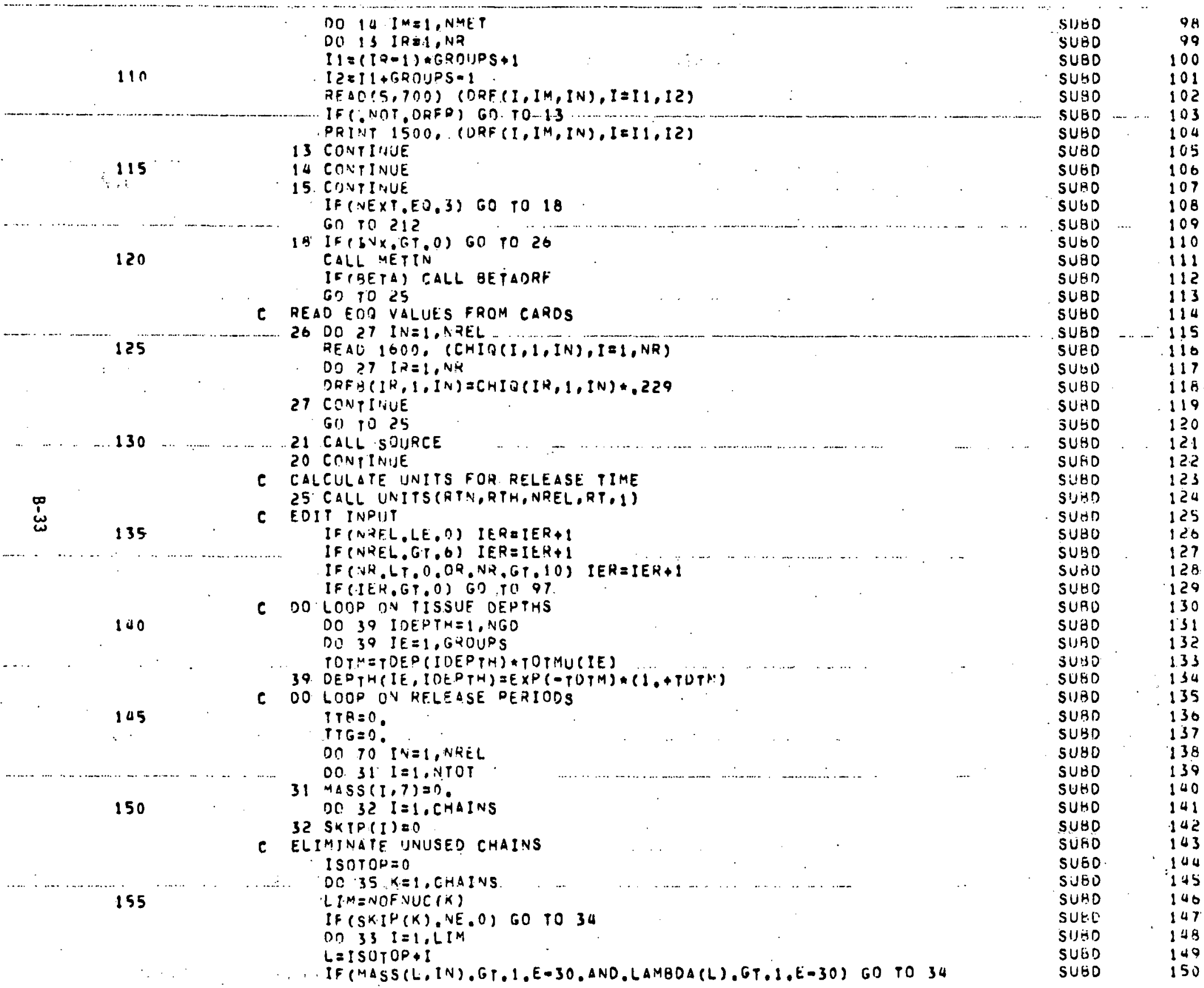




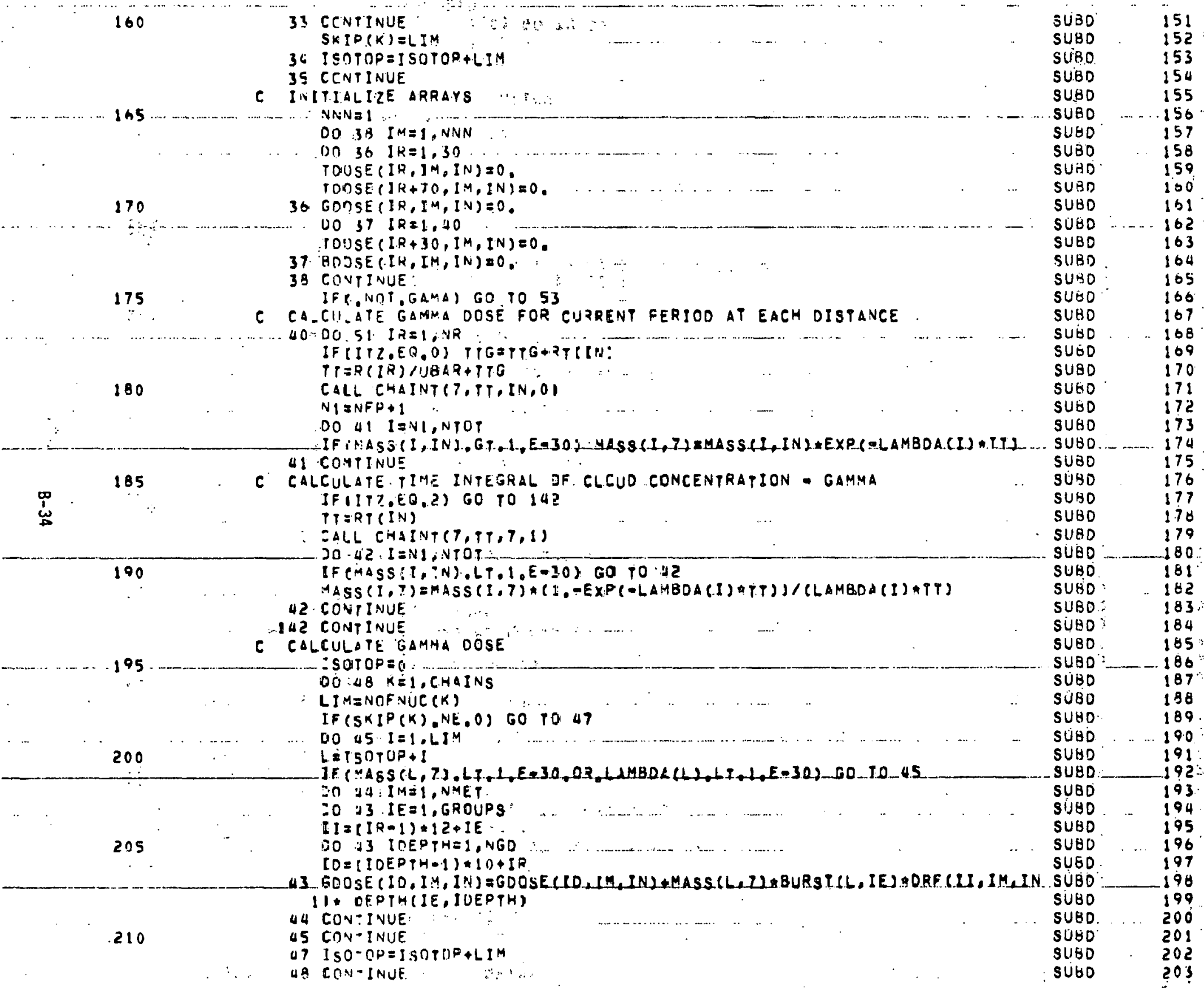




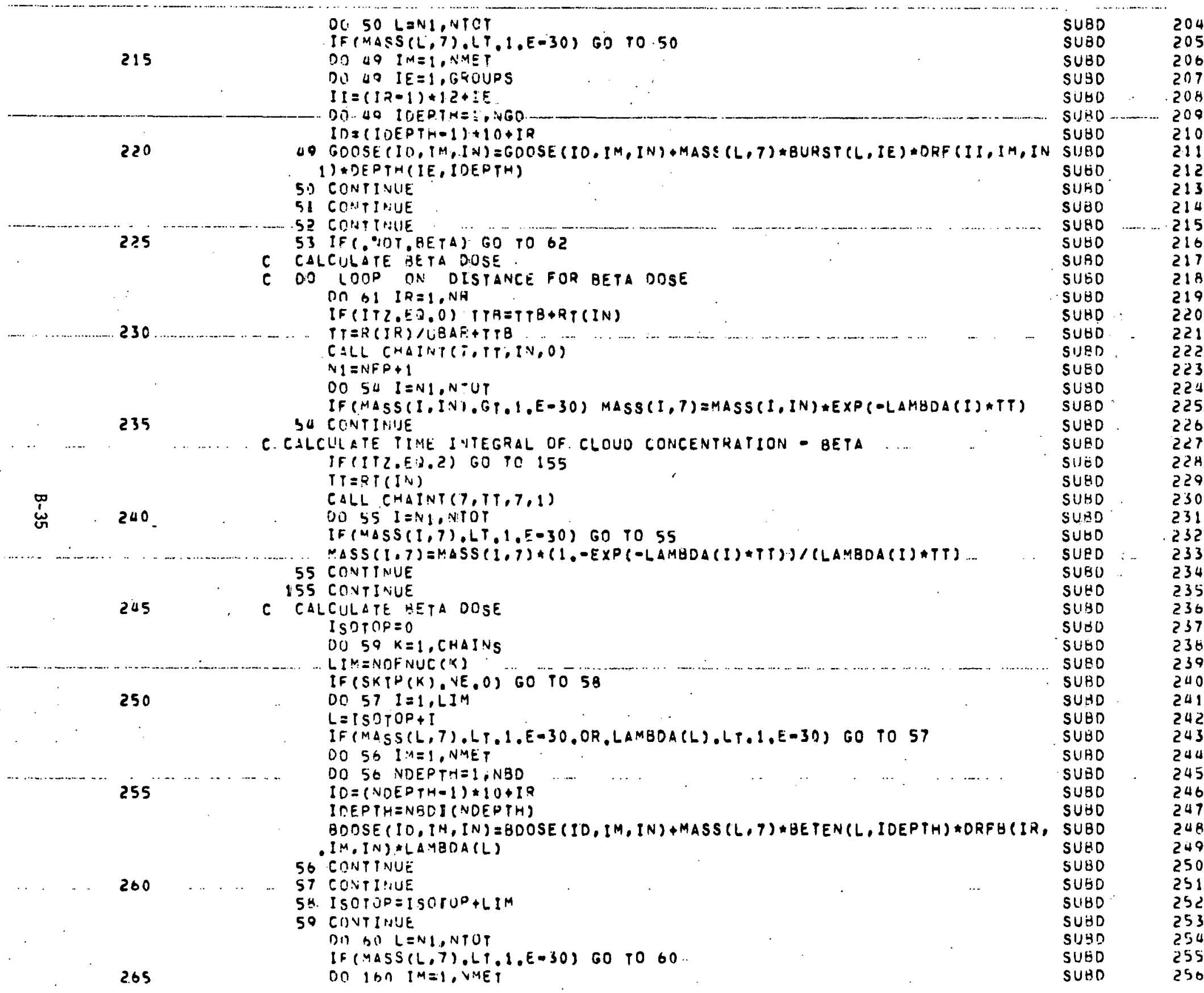




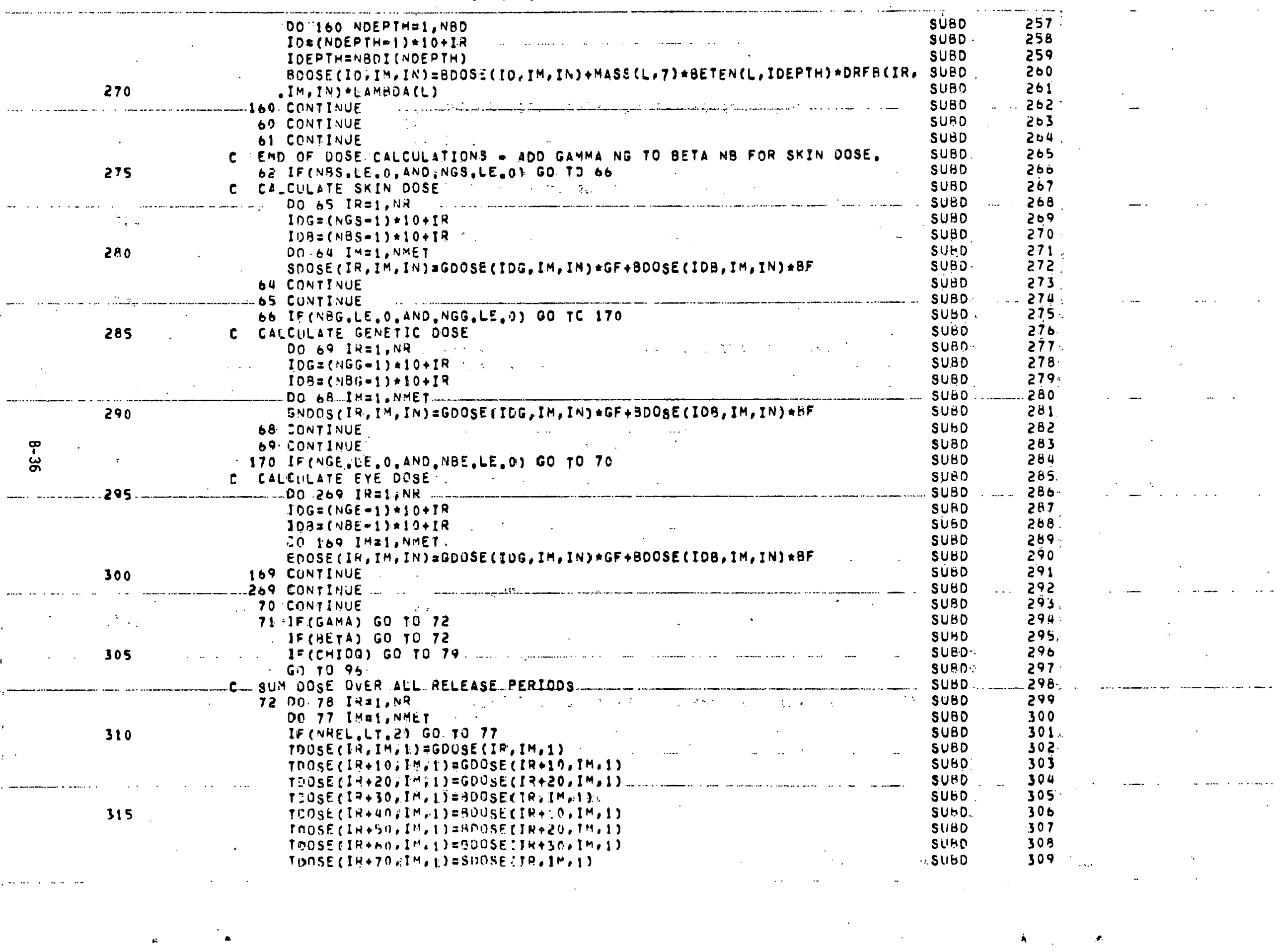




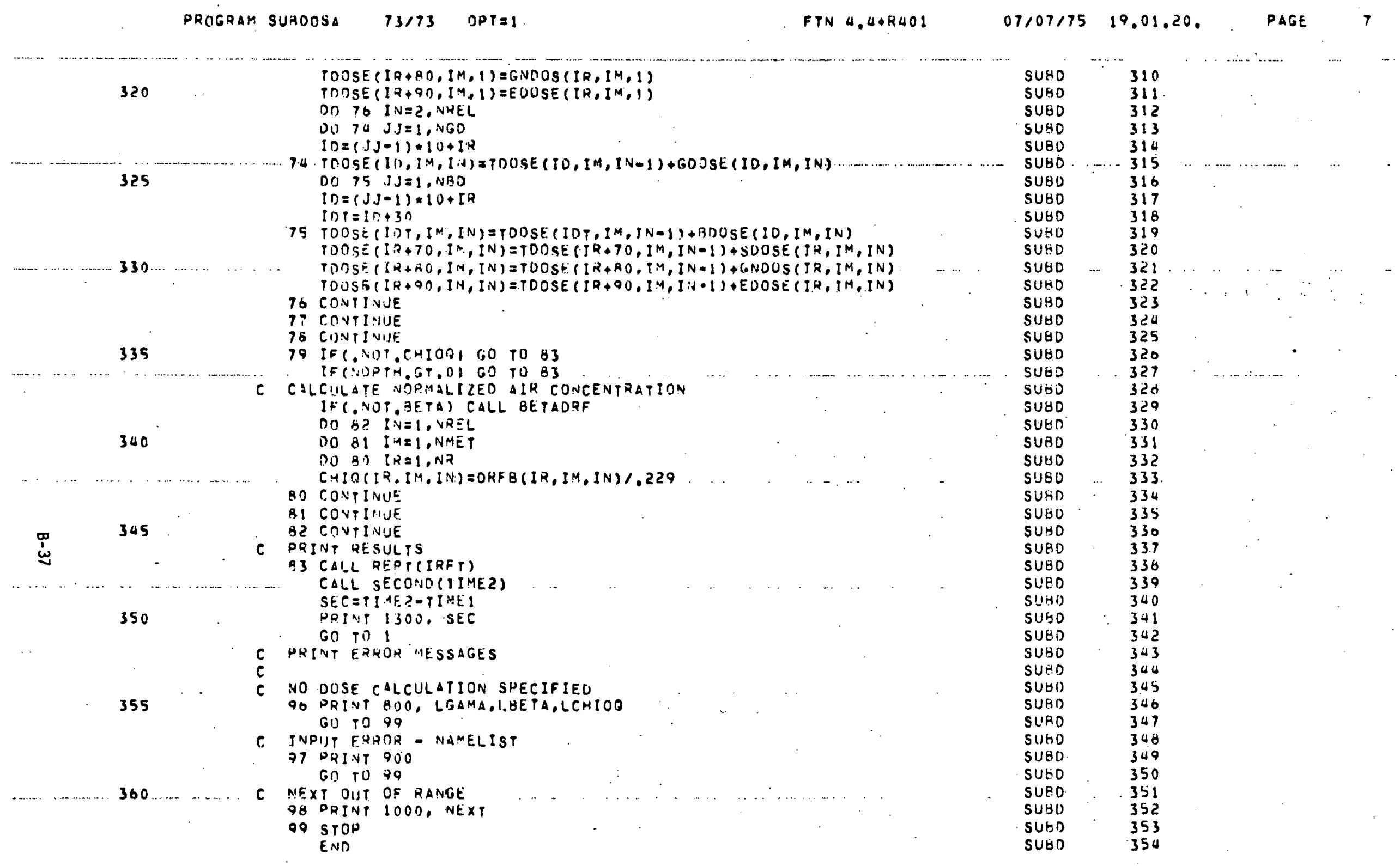




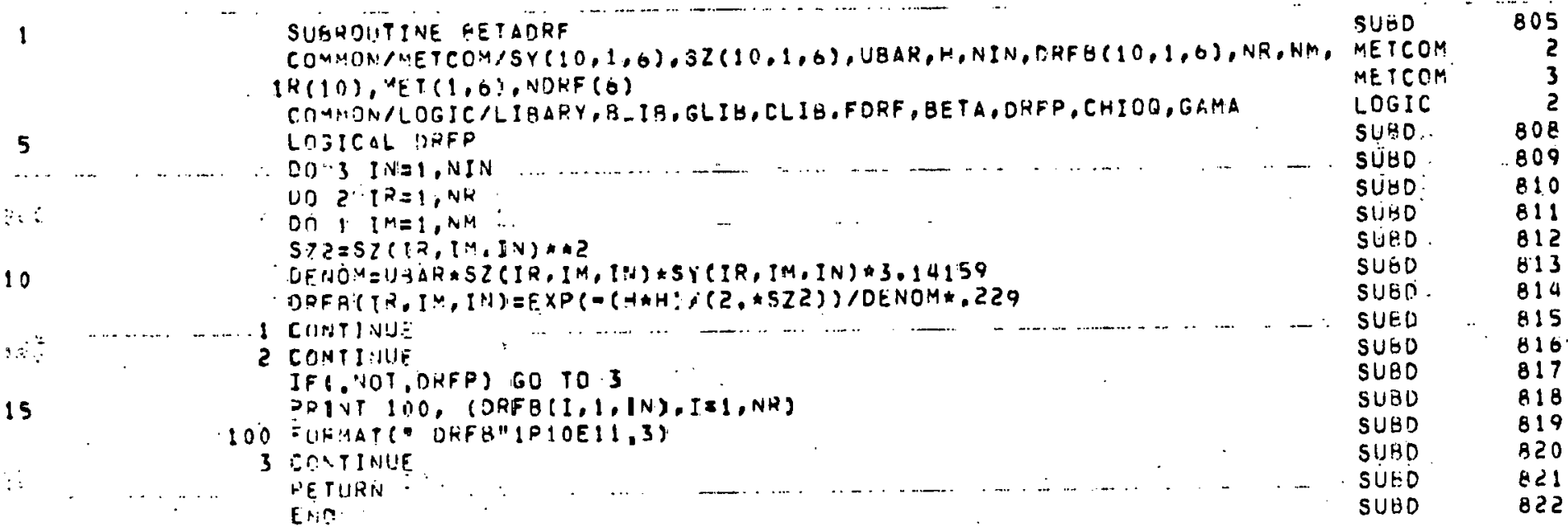




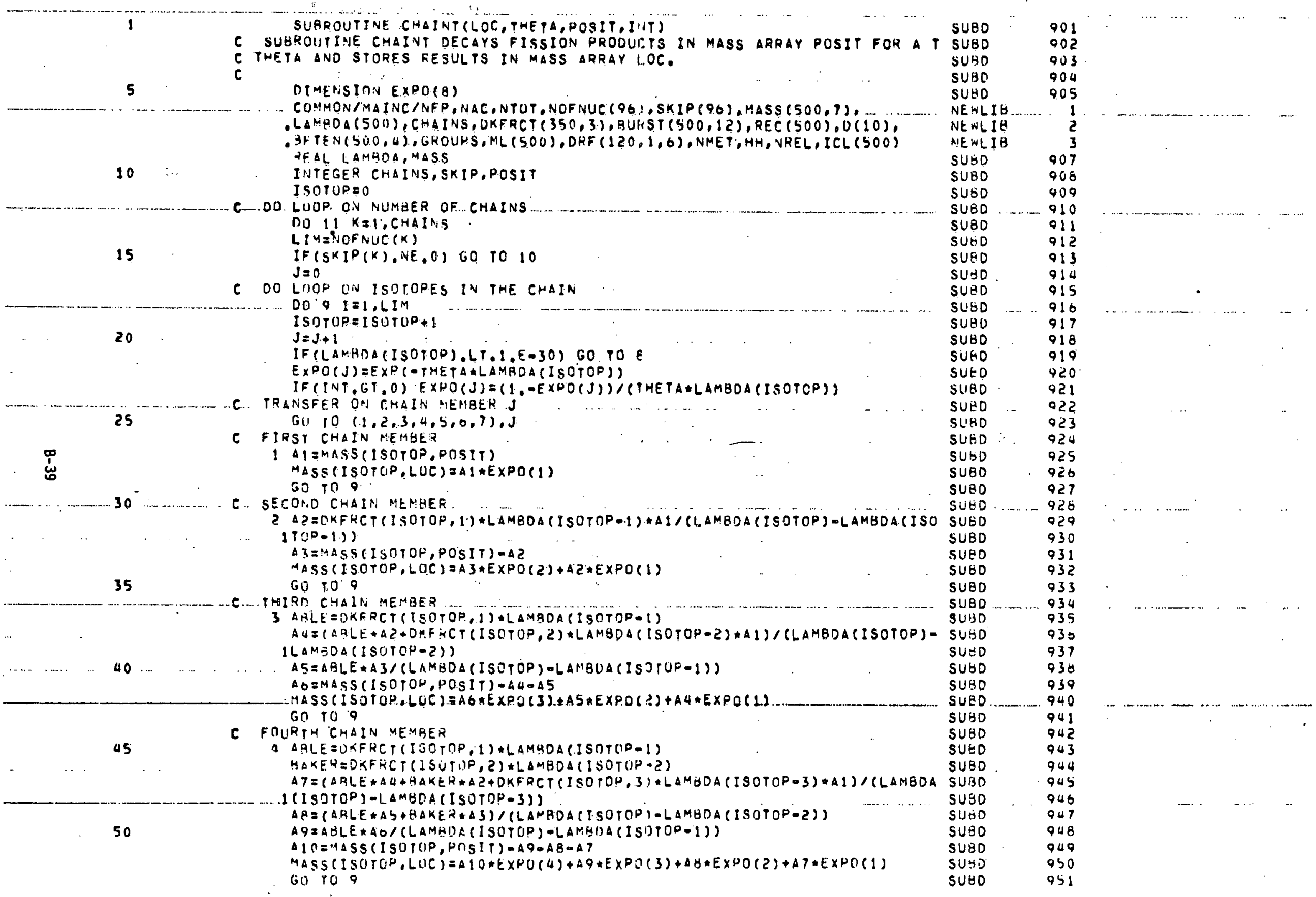




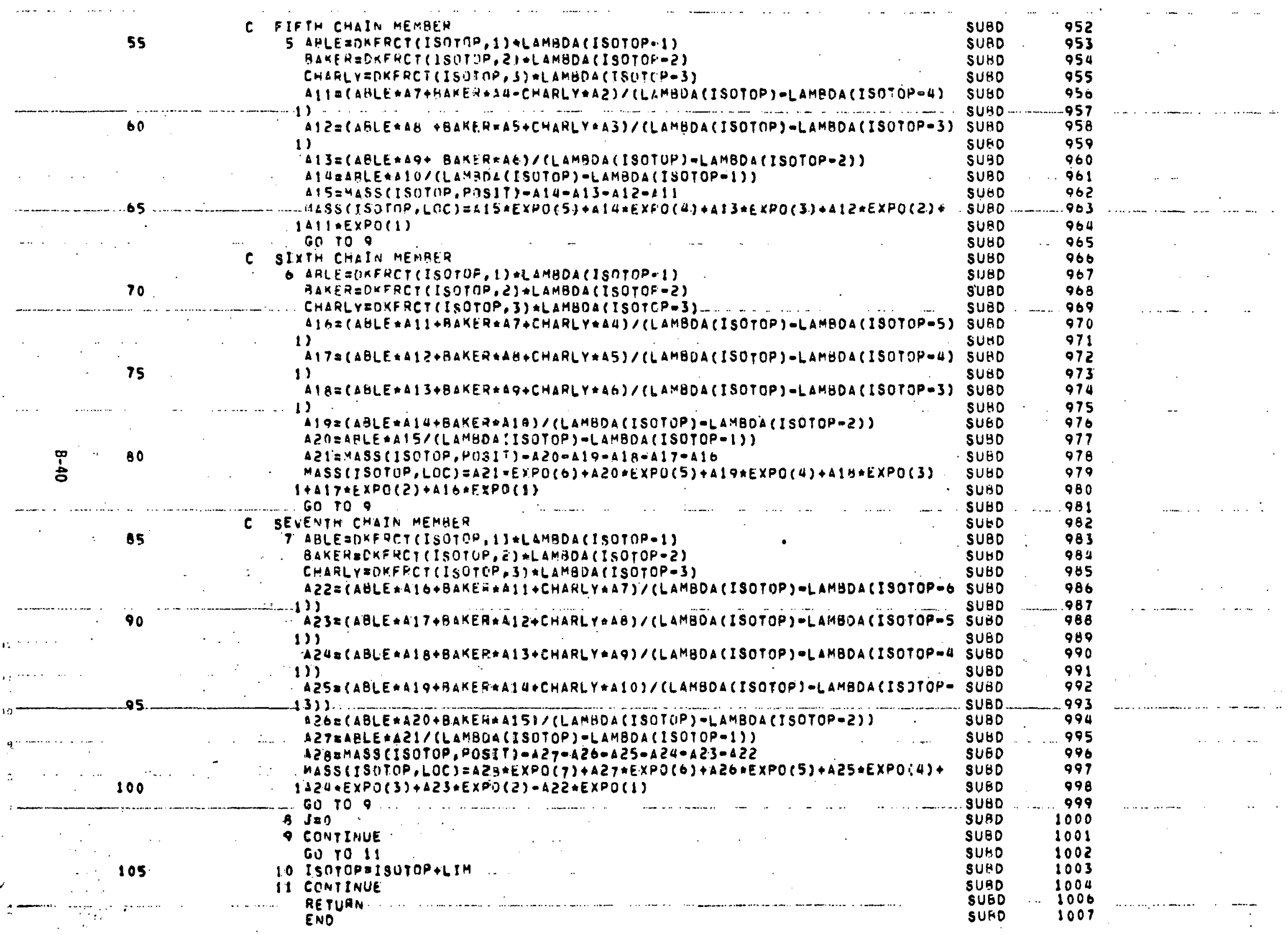




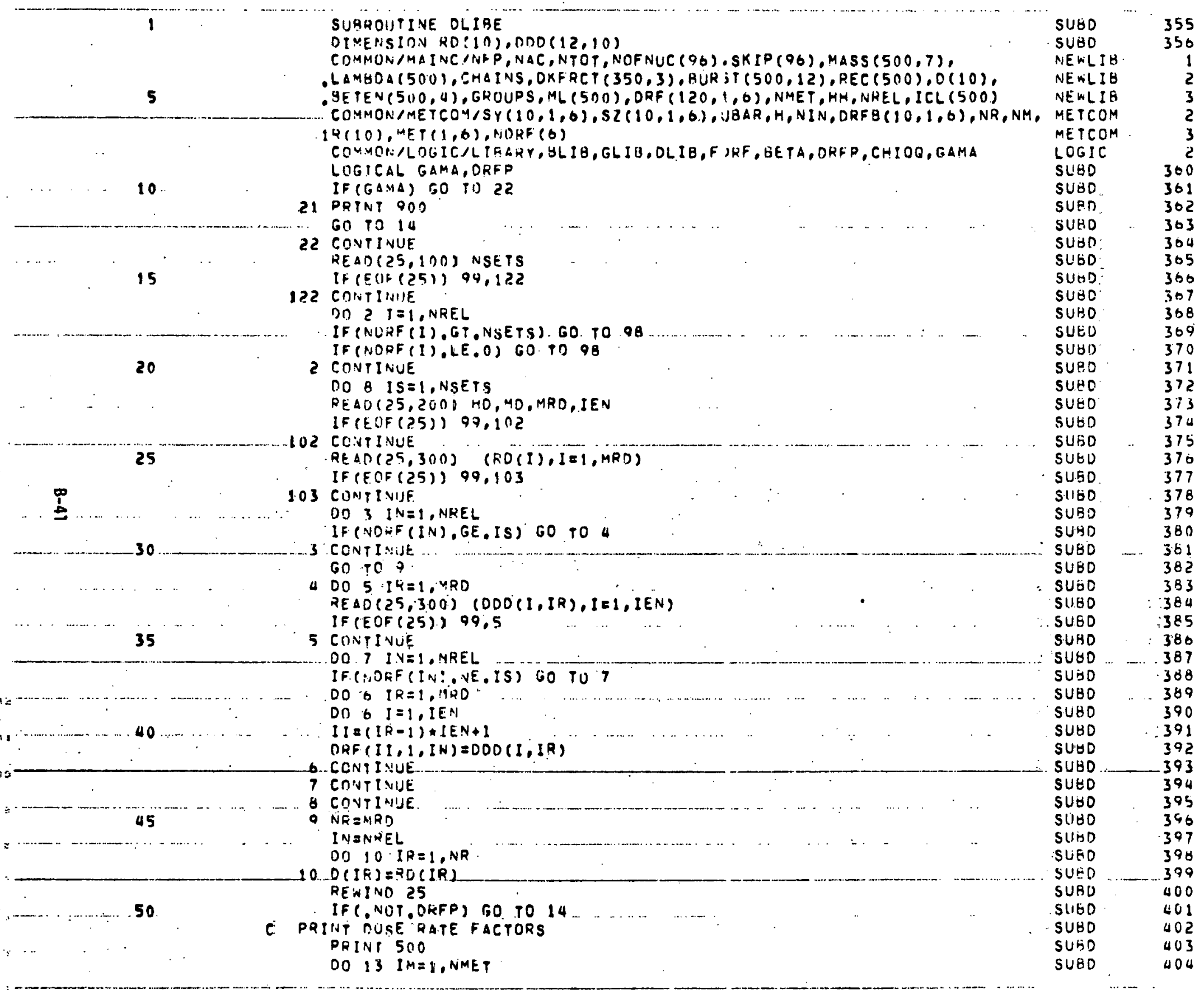




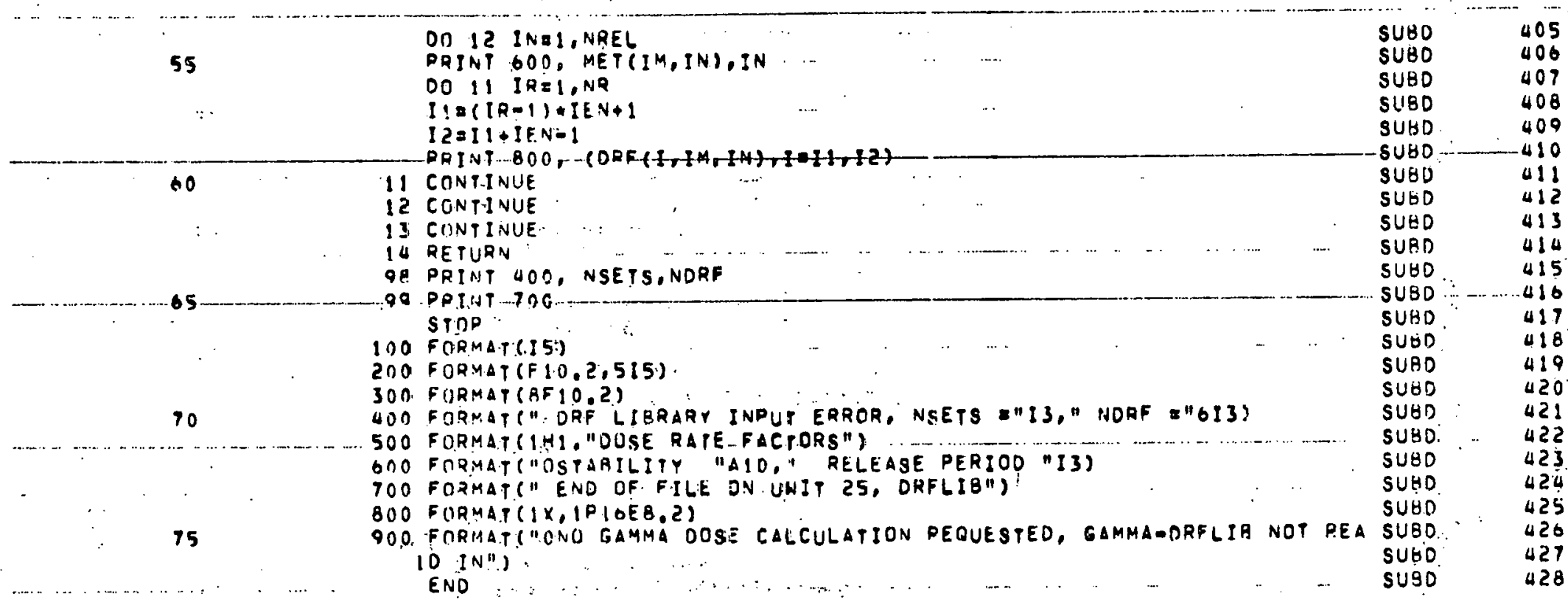


5

10

15

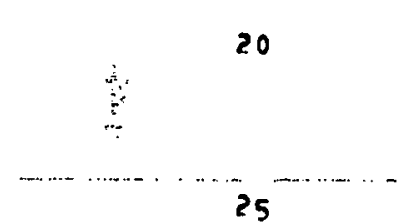

25

0
1
0

SUSROUTINE GLIHE

THIS SUEROUTINE HEAOS A PHOTON PROBAHILITY LIBRARY - GAMLIB - AND

CALCULATES BURST (ISO, IEN) FOR. EACH. ISOTOPE AND ENERGY TROUP.

a RADIONUCLIDE OATA LIBRARY MUST BE READ FIPST.

OIMENSION FRACT $(100)$, ENERGY $(100)$, MEV $(10)$, ENG $(16)$

DIMENSION KEV(:OO)

C(IMMON/MAINC/HFF, NAC, NTOT, NOFNUC (96), SKIP (96), MASS $(500,7)$

LAMRDA (5OO), CHA [NS, OKFRCT $(350,3)$, BURST $(500,12)$ REC $(500), D(10)$

- It TEN $(500,4), G P O U P S, M L(500), D K F(120,1,6), N M E T, H H, N R E L, I C L(500)$

RESL LAMADA

INTEGER IBRUPS

HEAL MEVIKEY

READ(?O,1) GROUPS

1 FORMAT (13)

KEV $V(520 ! J P S)=5.0$

$\operatorname{LiN}=600 \mathrm{NON}-1$

C KEV IS UPPER MEV LIHIT OF GROUP FOR LOWEP 15 GROUPS

TEDD $(2 A, 16)$ (KE

16. FORMAT(16F5.0)

(I), I 1, GROUPS)

MAT (aElo.0)

IF (GACUPS.GT.W 60 TO 316

ENG $(1)=.7$

GO 10616

31t IF (TRRUPS.GT.2) GO TO 416

$E N G(1)=., 5 h 67 * K . E V(1)$

$E N(,(2)=1.2 * K E Y(1)$

GU TO 616

41.6 FA(G(1) $=.0067$ \#EV (1)

ENG (r,ROUPS) $=1$. 2 KE V (GROUPS- 1 )

$L=$ GRO11PS - 1

$D O 510 \quad I=2, L$

51. ENG (I) $=(K E V(1)+K E V(1-1)) / 2.0$

DIS CONTINUE

DO 7.16 I $I=1$, GROUPS

35

$\therefore$ READ(DA, InO) NCD.isos

1On. FOQMAT(2I3)

DO 23 ISOTID $=1$. NTOT

$40 \ldots 00$. . . $11 \mathrm{~h} L=1.1 \overline{\mathrm{c}}$

116 3!IRST (ISOTOD,L) $=0.0$

10 READ (2B,1

1.7 FORM.A $(3 x, 12,3 x, 12,12 F 5,0,12)$

$N[I M=N]$

SP.T $V=0$

IF (NC,MT,LE,O) GO TO 217

45

DO 117 JJJ $=1, N C: M T$

$1: 7$ HEAOC 28,200$) N C+T$

200 FOHMAT (AL)

G0 $10 \quad 316$

517 ICL $(12 D T$ TIO) $=4$

IF (N.EO.IA.UR.N.EO, 36.OR,N.EO. S4) ICL (ISOTOP) $=1$

IF (N,E'T, \{4, IIR,N,E?, 52,OR,N,ER, 55) ICL. (ISOTDP) $=3$

SLBD

SLBD $\quad 599$

SUBD 600

SUBD $\quad 601$

SUBD $\quad 602$

SUEO 603

SUSO

NEWLIE

NENLI 8

NE PLIB

SURD

SUED

SUBD

SUBD

SUBD

SUBD

SUBD

SULO

SUbio

SUID

SUBD

SU8D

SUSD

SUBD

SUED

SLED

SUAD

SUO $0^{\circ}$

SUED

SUSO

SUBD

SUS̄o

SUSO

SU30

SUnO

SUBD

SUED

SULD

SUBD

SUSD

SUSO

SUED

SUBD

SUSD

SUBO

SUED

SUED

SUHO

SUSO

SUED

SUEO

SU⿺D

SUEO

SUSD

SUSD 


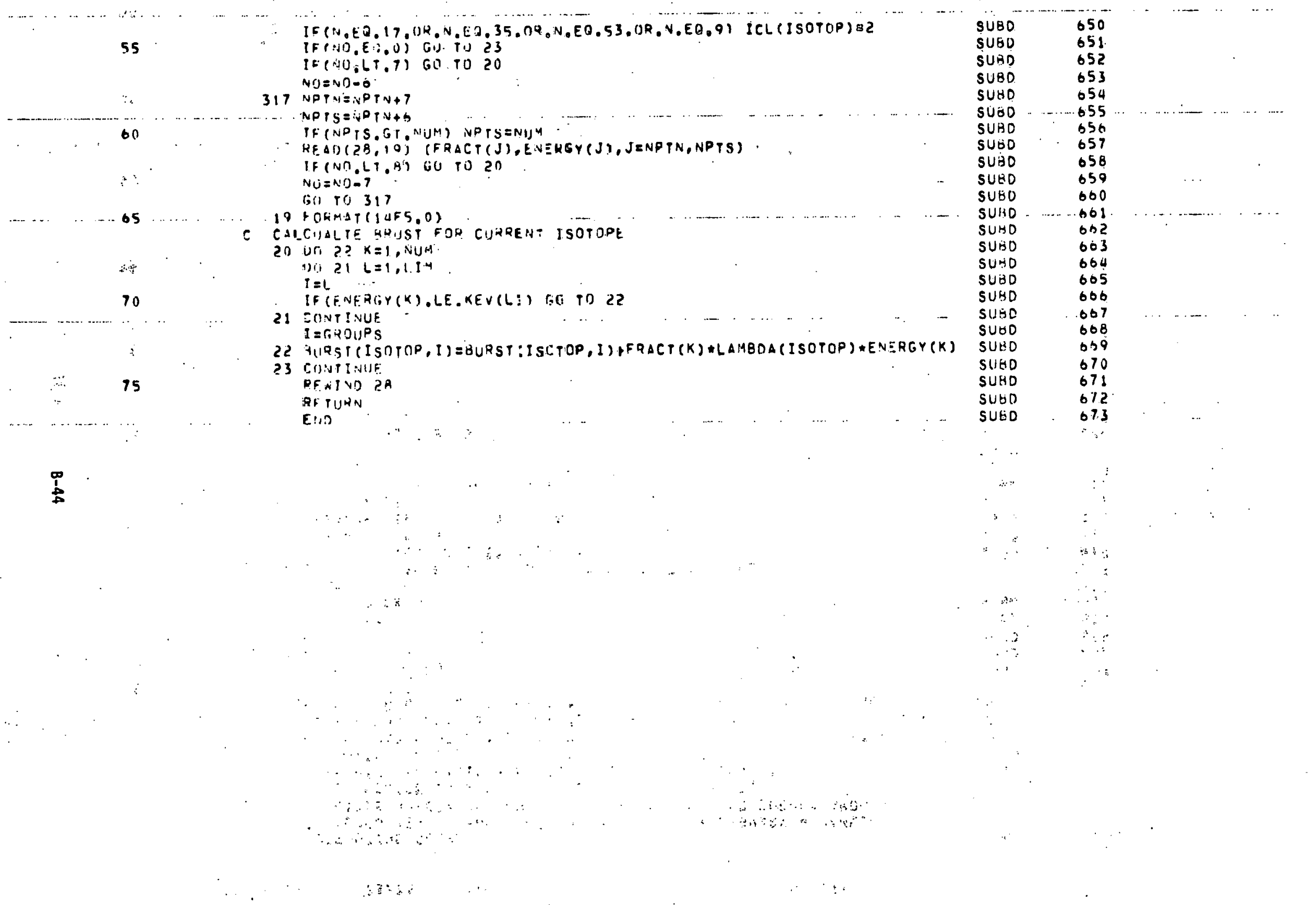


SURDOUITINE LIEK

SUAROUTINE LIBK READS A RADIONUCLIDE DITA LIBRARY. RNDLIB, AND

SUBO

482

C CALCULATES DKFRCT(FRACIIONAL YIELO FROA: DARENTS).

SUMD

483

OIMENSION FRACT $(350)$, ISO $(500)$, NA $(500)$

COMBAIN/AAINC/NFD, DAC, NTOT, NOFNUC (96), SKIP(96), MASS $(500,7)$

NE WLIB

- LAMANA $(500)$, CHAINS, DKFRCT $(350,3)$, PUFST $(500,12)$, REC $(500), D(10)$

HETE: $(500,4), G G O U P S, M L(500), O R F(12 n, 1, b), N M E T, H H, N R E L, I C L(5 \cup 0)$

NE. $\triangle 18$

NEWLIB

INIEGER CHAINS

10

REAL LAMBDA

NEXLLIG

SURD

SUBD

SURO

c...

15

READ $(20.1)$ NCO

1 FPRMAT(15)

SU⿺辶⿻

SUED

RFAD(20,5) (ML (J),NA(J),REC(J)ISC (J),LAMBDAC(J), FRACT(J).

SUE

( $Q E$ TEN $(J, 1), I=1,4), J=1, N C D)$

$2 / 5,5 / 72$
$N E R L$

$5 / 221750$

FORMATCI3,I2,A3,11,EY,1,57,4, 4E10,2

NENLIB.

$5 / 22 / 75 \theta$

20

102 FORAAT(I3,12, $3,11, E 9,1,7 \times, 4 E 10,2\}$

READ(29, 1$)$ Ne

$N i=N C D+1$

N\&C $=\mathrm{NC}$

$N 5=4,5+N C O$

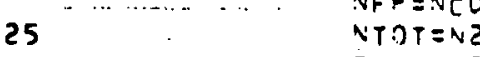

RE $\triangle O(29,102)$ (ML (J),NA(J),REC(J), ISO(J),LAMBDA(J),

SUBD

SUED

SUEO

SUBD

SUTO

(SE, IEN $(J, I), J=1,4), J=N 1, N 2)$

D. DO 20 ? L LNI, N2

IF(LAMBC.A(L),LE, I.E-30) GD TO 202

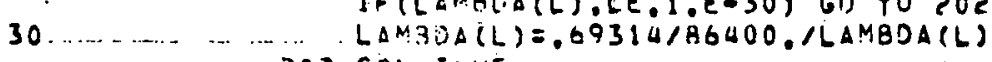

502 CONTINUE

RENINU ZO

ERROR=.FALSE:

$M P=0$

35 CHAJYS $=0$

UQ $3 \quad I=1,96$

3 NiTENUC(I) $=0$

C CALCIJLATE LA!MBDA IN SEC-I

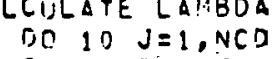

LO IF(LAMGDA(J).LE.I.E-30) GO TO 104

LAMBOA J J $=.69314 / L A M B D A(J) / 86400$.

SUHD

$5 / 22 / 7.5$

$5 / 22 / 7.5$

SUSD

511 i 0

SUBD

SIJED

SUED

SUSO

SUED

SUHD

SUEO

SUBD

SUBD
SUPO

SUeD

SUBO

SUSO

Cuivtisue

IF (MP.ML (J)) 4.204 .6

45

$2(14$ If $(N A(J)-N A(J-1), L T .2)$ GO TO 5

$4 \quad Y P=V L(j)$

C NEW MASS CHAIN

CHOINS = CHAINSTI

C COUNT NUAGEH OF VUCLIDES IN THIS CHAIN

SUED

NEWLIS

NEMLIG

SIJBD

SU⿺辶

sijigo

5 NOFNUC (CHAINS) aNOF, NUE (GHATNS) + 1

NOFNUC

- ERRLR= TRUE.

DDINT T,J

7 FURMATC"OLIHRARY ENTRY"I4," IS OUT OF PLACE")

SUTD

SUHO

SIIRD

SUEO

SUBD

SUBD

485

3
2
3

498

489

490

$49 !$

493

5

1

497

490

501

502

505

$50 \mathrm{n}$

507

508
509

510

511

512

513

515

516

517

518

519

9
10

5?!

522
523

524

525

520

527
528

52.9 


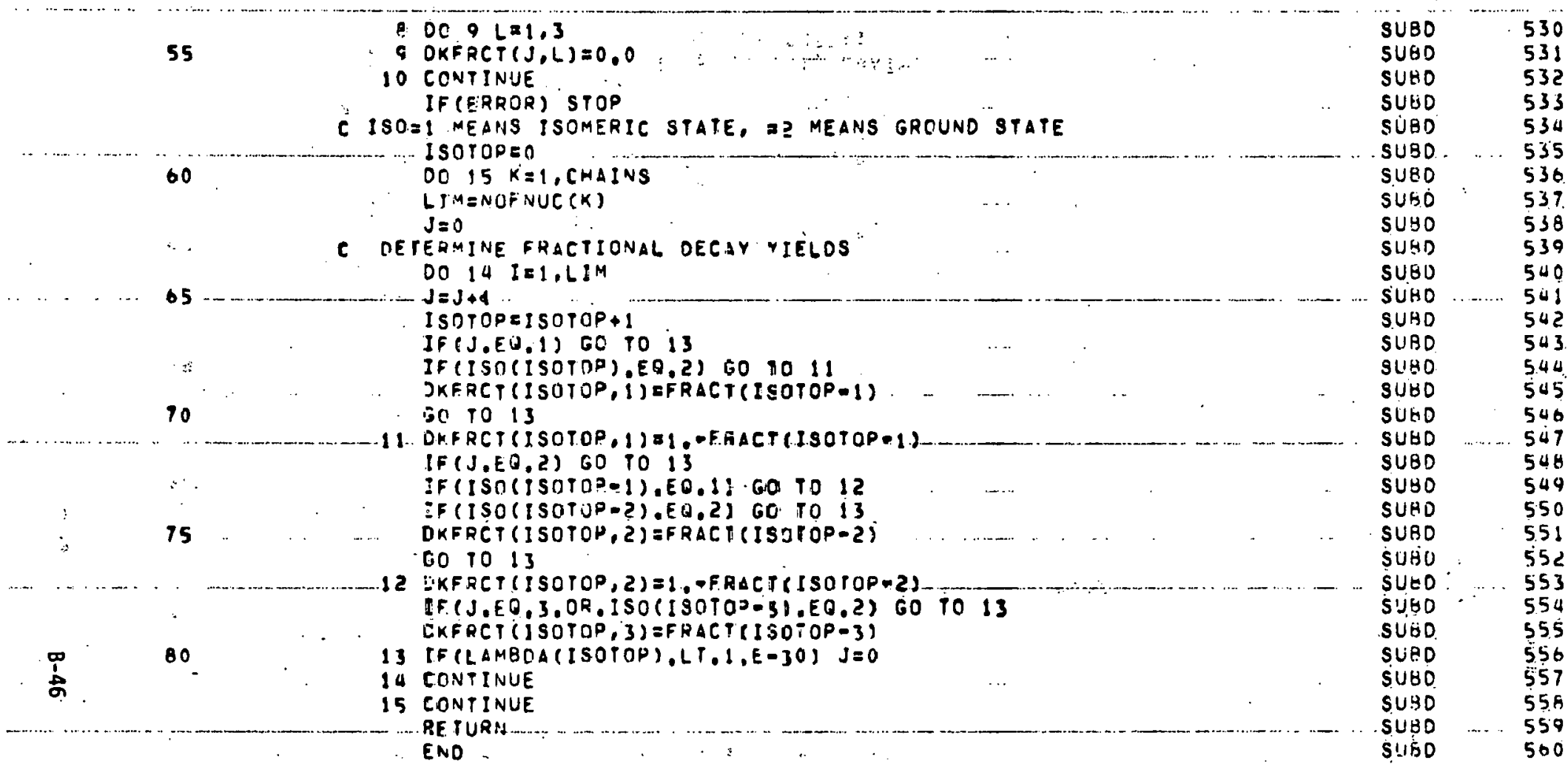




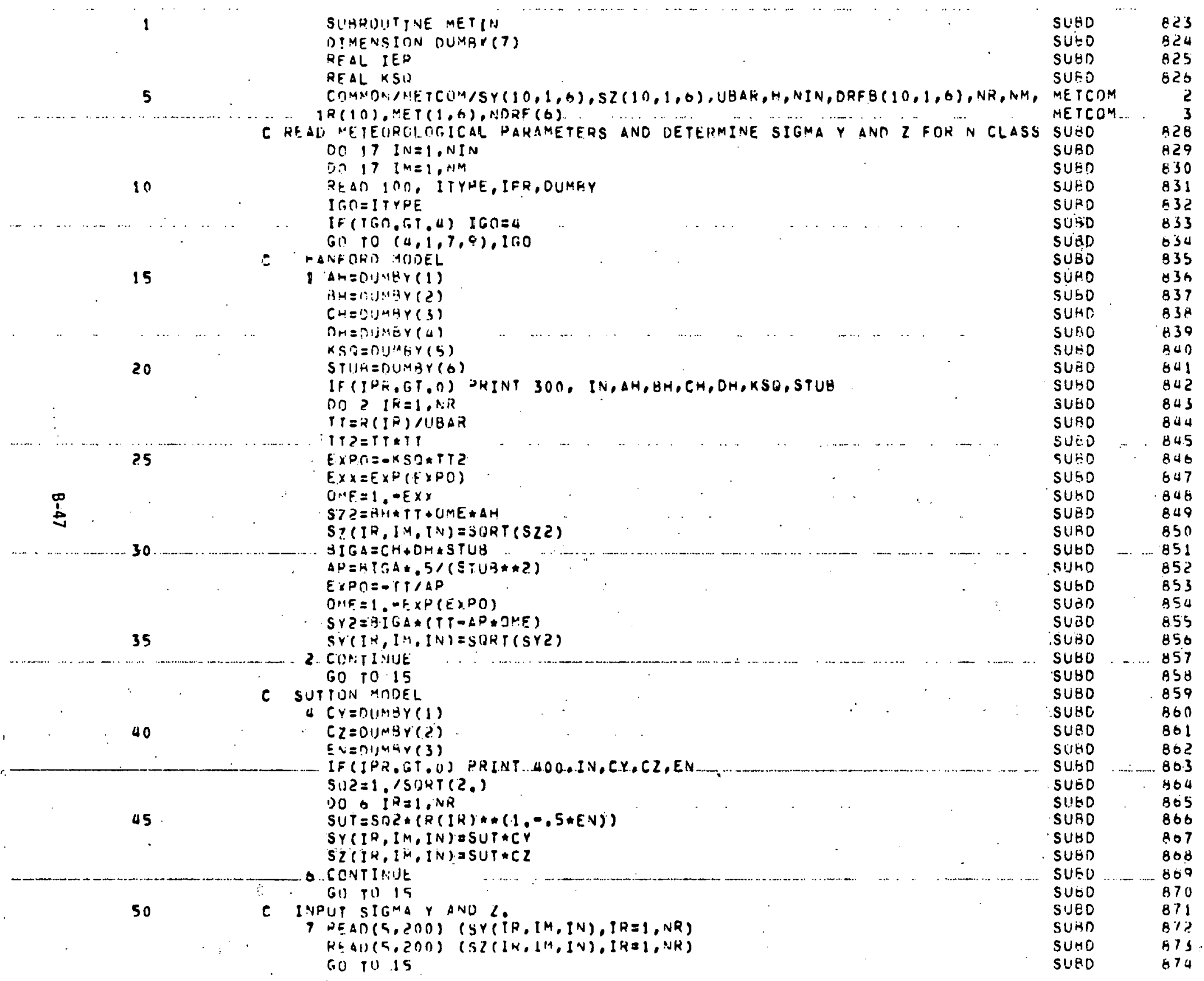




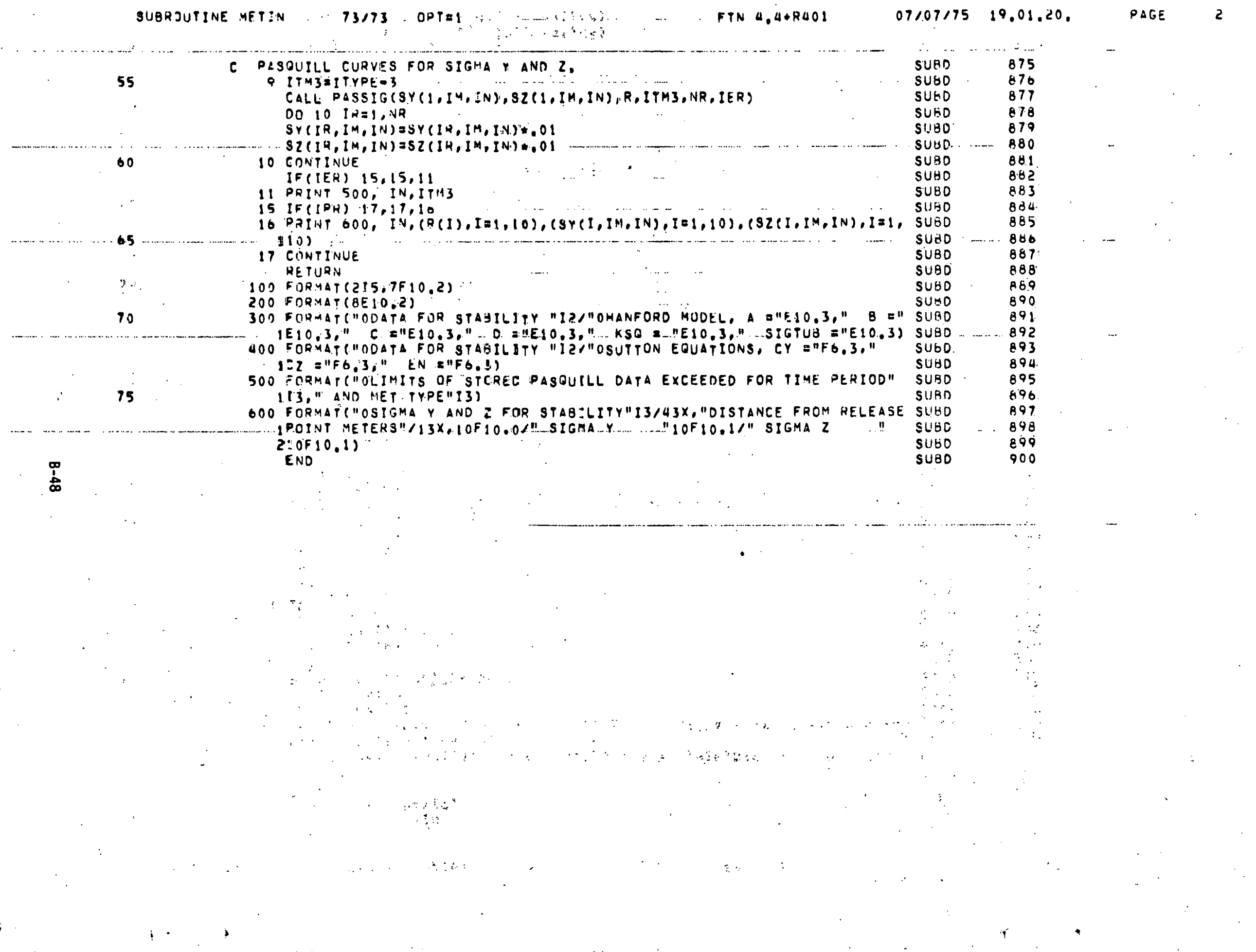





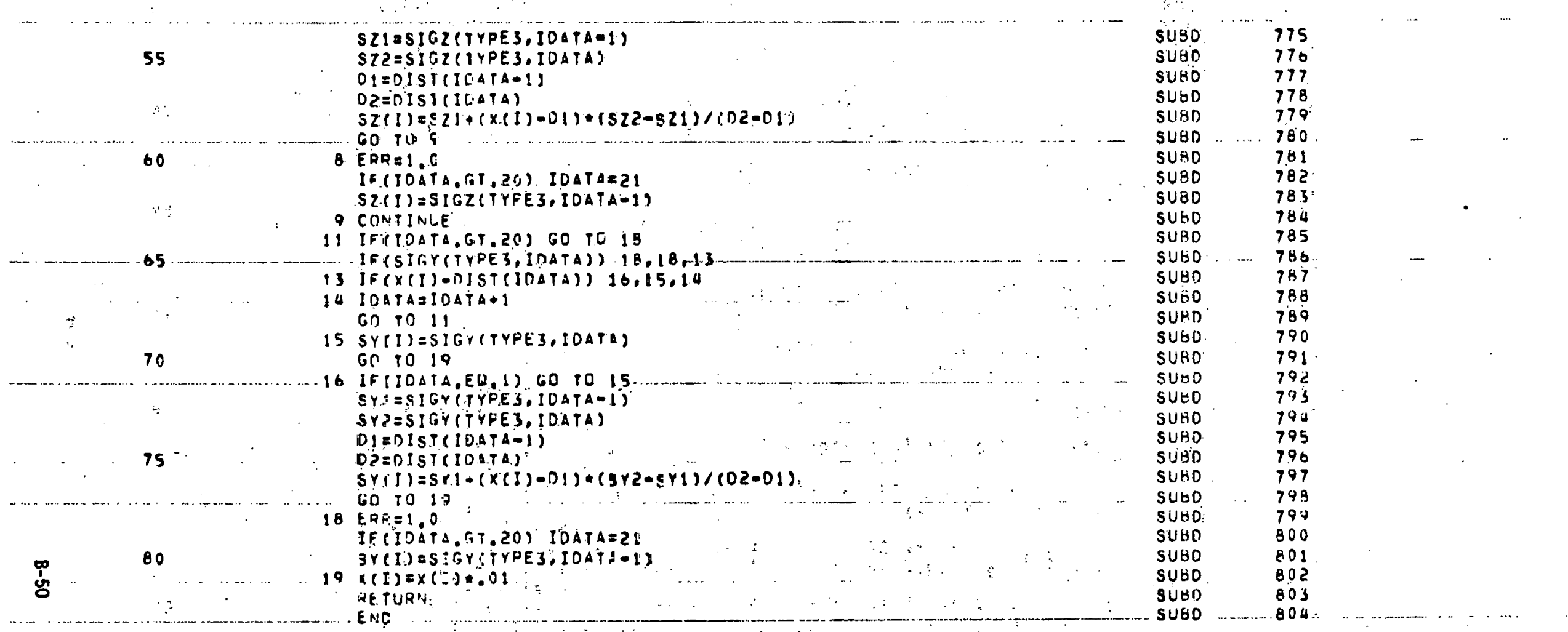




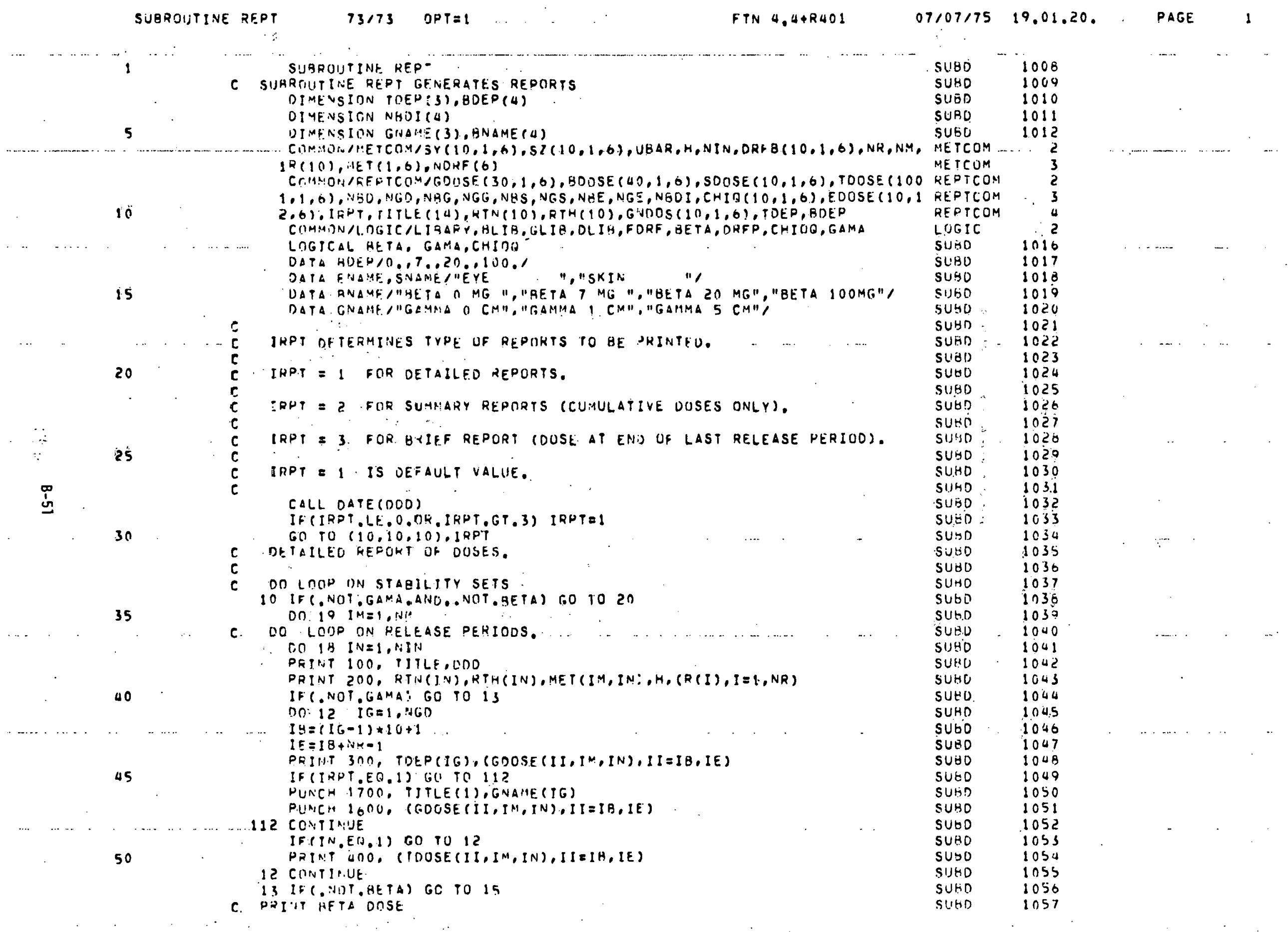


$I 1=I 1+30$

.65

$12=12+30$

\section{PEINT 400 , (TOOSE III,IM,IN),IIIII,IZ)}

14 CONTTINE

c print SKIn DOSE

15 IF CNAS.LT.1.AND.NGS.LT.1) GO TC 10

70

PUINT GNO: (SDOSE (I, IM, INI, I $=1, N R$ )

IF (TRPT.ED. I) 60 TO 116

PloNer liou, TITLE(1),SNAME

P(INCC loDO, (SOOSE(I.IM,IN),I=I,NR)

75 I16 CONTISUE

$I_{1}=71$

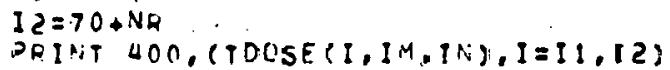

$I Z 2=70+N R$
DPIPT $400,(T D O S E(I, I M, I N), I=I 1,12)$

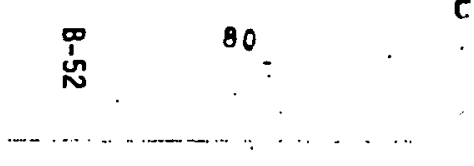

WRTHT EYE ONSE AND.NGE.L-.1) 60 TO 17

NRINT.TOO, (EOOSE (I, IM, IN), I=1,NR)

if(IPET.FO.1) OC TO 117

Plinea 17no; ritle(1),ename

85

OHACH loOD, (EDCSE $[1] M,, I N), I=1, N R$ )

17 CENTINUF

if (IT,Eo,i) an tall 17

i $11=91$

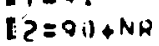

90

PRINT YOO, (TOUSE (I, IM, [NI,IIII, I 2$)$

C PRINT GENETIC DOSE.

17 IP NNHG.LT.1.AHG.NGG.LT, II GO TO IIB

PRINT BOO, (GINOCS(IIIM, INT, I = I, NR)

if (IN.EO.S) - GU 90.118

i. $1=91$

$12=80+N 8$

9.5

DHINT 400

(TOUSE (I,IM, IN], [aII,IZ)

Fin isit isio

I: (N.HS.LT.1.AND.NTS.L.T, 1) GO TO. 19

100

FETNT IZOO, TREP(VGS), EDEN(NRS)

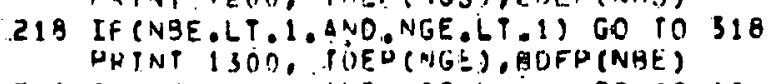

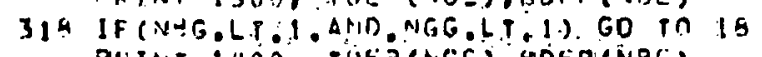

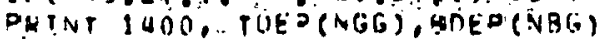

1. Continde

iq ChNilinut

SUEO

SUBD

SUBO

SUQD

SUED

SUBD

SUEO

SURD

SUED

SUBD

SUtio

SURD

SUBD

SUBD

SUOD

SUEO

SU80

SURO

SUGD

SURD

SUED
SUAO

SUAO

SULO

SUFO

SUEO

SUBO

SUBD

SUIO

SUED

SUED

SUgo

SUBD

SUED

1058

1059

1000

1001

1062

1063

1064

1065

1066

1008

1069

1070

1071
1072

1073

1074

1075

1076

1077

1078

1079

1080.

1081

1082

1083

1084

1085

1080

1087

1088

1089

1090

1091

1092

1093.

1095

1096

1096

1098

SUED _._. 1099

SUED 1100

SUED 1101

IIOC

SUBD: 1103

1104

SUED … 1106

SUAD 1107

SUISD $\quad 1108$

SUED $\quad 1109$

SUHD 1110 
C PHINT CHIJO VALUES

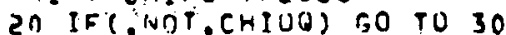

NOTNT 10O,TIT-E.ODD

DEINT $900, H,(R(T), J=1$, NR

SUED -.. ......

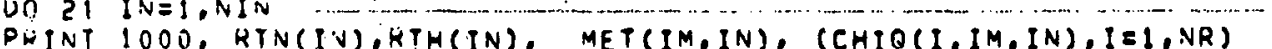

SUBD

SUito

1111

DO ZI IN=1,Nin

51 CONTINTE

115 PQINT 1100

2? CONTINUE

C. HEADING FOHMAT FOR DETAILED DOSE REPORT 100.FORMAT("I"13A6,A2, POX, "DATE "A10)

120 DISTANCE METERS" PERIOD, STABILITY "AIOR SUEO "DISTANCE, METERS"1OX, "RELEASE MEIGHT"FS.0 SUBD

$10 F(1.0)$

GDMMA OSISE FOQ:12,

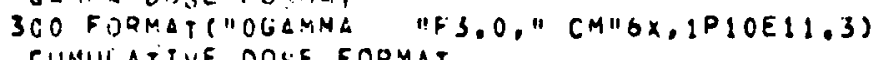

UCOFFOMAT(" TOTLL DOSF. THIS TYPE"IPIOE 11,3)

125

C DETA DOSE FORMA:

SODFOPMAT("NEETA "F4.0," MG/CMZ "IPIOEI1.3)

C SKIN OOSE FORMAT

OOO FDDAA I ("O5KIWIIOX, IPIOE!1,3)

130

C EYE DOSE FDRUAT

TON. FURMATS"OEYE"17X,1P1OE11,3)

C GEVETIC UOSE FORMAT

Gi) FORHAT ("OCENEIIC" $13 x, 1 P 10 E$ 11.3)

罚 C CMIIOTITLE FURMAT

QTO F MRMATLUOX, INORMALIZEO ATP CONCENTRATION" " IIGHT "F5.0."HETERS"/21X, IOF11.0) 1000 FORMATCIX.FU.1,AS, 1X,A10,1PIOE 11.3

$$
1 \text { COSE." }
$$

5.1." CM GAIMA DOSE +"F5.0," MG/CMZ BETA SUIJO

$\$ 144$

1300 COSE."

114.5

1146

$1: 47$ 1 OOSE.") :

145 1 COSE.")

1500 FORMAT(1)

1600 FIIRMAT (1CEQ.3)

1700 FURMAT (A5,A:0,"1")

30 RF TURA

ENO 
CONTZOLS LIBRARY REAOING AND GELEASE RATE SPECIFICA SUBD

SIIRROUPIAL SOURCE

10

DIMENSIUN TITL(IA)

DIMENSIUAT UUAHTY(ODJ)

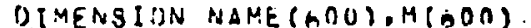

COHMON/MAINC IIIFP MAC NTOT NOFHUC (00), SKIP(06), MASS $(500,7)$,

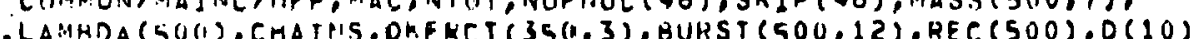

- HF TEN 500,4$)$, GROUPS, ML: $5(0)$, DRF $(120,1,6)$, NMET, HH, NREL, ICL 500 )

RFAL LAMHARA,MASS

INTEGER REC

10

e

i FORMAT (I3)

PRINT 300

15

$\because ;$

20

3 CO FURMAT (HI")

$4 \cdot D(; 17, I N=1, N R E L$

READ IOC, TITL

103 FURMAT (1SA6,A2)

20) FORMAT ("O" $1340, A Z)$

OO $104 \mathrm{C} T=\mathrm{l}$. Nitit

IOẢ MASSTI, INI)O:

NE.WLIE

NE WLIB

SUBD

SUED

SUSD

SUAD

SUED

SURD

SUKD

SUOD

SUBD

SUBD

SUED

SURD

SUBD

25

C WFAD NUCLIDE INVENTOKY FROM CAROS

10 KEAD l, PIUI:BER

REAO 11, (PAME (I),M(1),OJANPY (1), I=1, NUMBER)

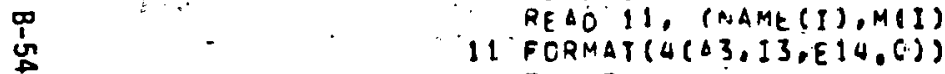

PRINT 0 , IN

30

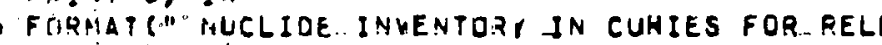

PRIATT 7 , (AIAME (I), M(I), DUANTYII), I I INUMBER)

c

I FnKMAT(4) 2XA3,13,1PE10,2)

35

TIY 10 IDENTIFY NUCLIDES RZ DO FRCIM CARUS

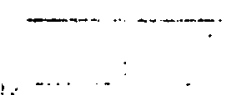
OO $16 \quad I=1$, NUMBER

$D B$ i $3 \mathrm{~J}=1$, NTO

IF (NANE(I),EQ.REC (J),AND.M(I) ,EO.ML (J) 60.70 .15 SUSD

15 CÓNTINUE.

00

IF CAN NOT IDENTIFY NUCLIDE -STOP

PRINT I L, NAME (I),M(1)

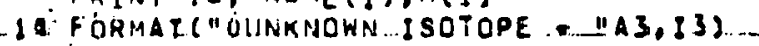
STOP

45

C CCNVERT CURIESTTO CURIE-SEC

15 IF (LAMHOA(J).LE, I.E-JO! GC TO 16

MASS (JIIN) G QUANTY (I)ILLMECAC(J)

10 CONYINUE

1 CONTINUE

50

RETURN

END?

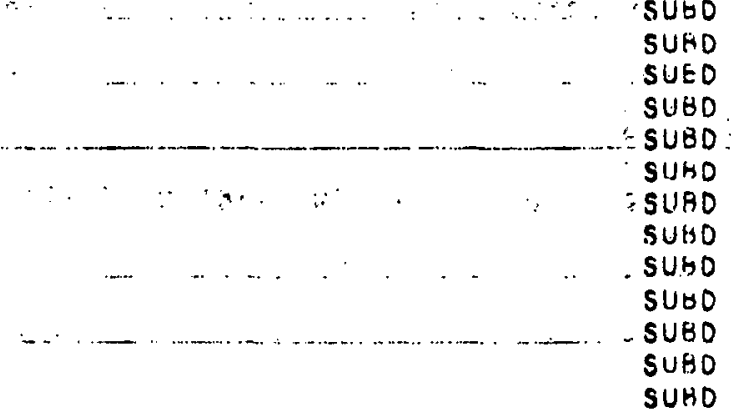

SUSD $\quad 677$

SUBD

NEWLIB

SUSD

SUBO

SUBD

SUBO

SULD

SUBO

SUBO

SUBO

SUED

SUGD

SUBD

SUAD
SUSO

SUED

SUBD

SUHD

SURO

SU⿺辶

SUBD

SUBO

SUHD

679

$\therefore 2$

68

682

1683

694

.645

686

687

698

... 689

690

091

.092

$.69,4$

695

696

.700

700
701

703

704

.705

700

708

709

710

711

712

.714

.715

716
717

718
719

720

721 


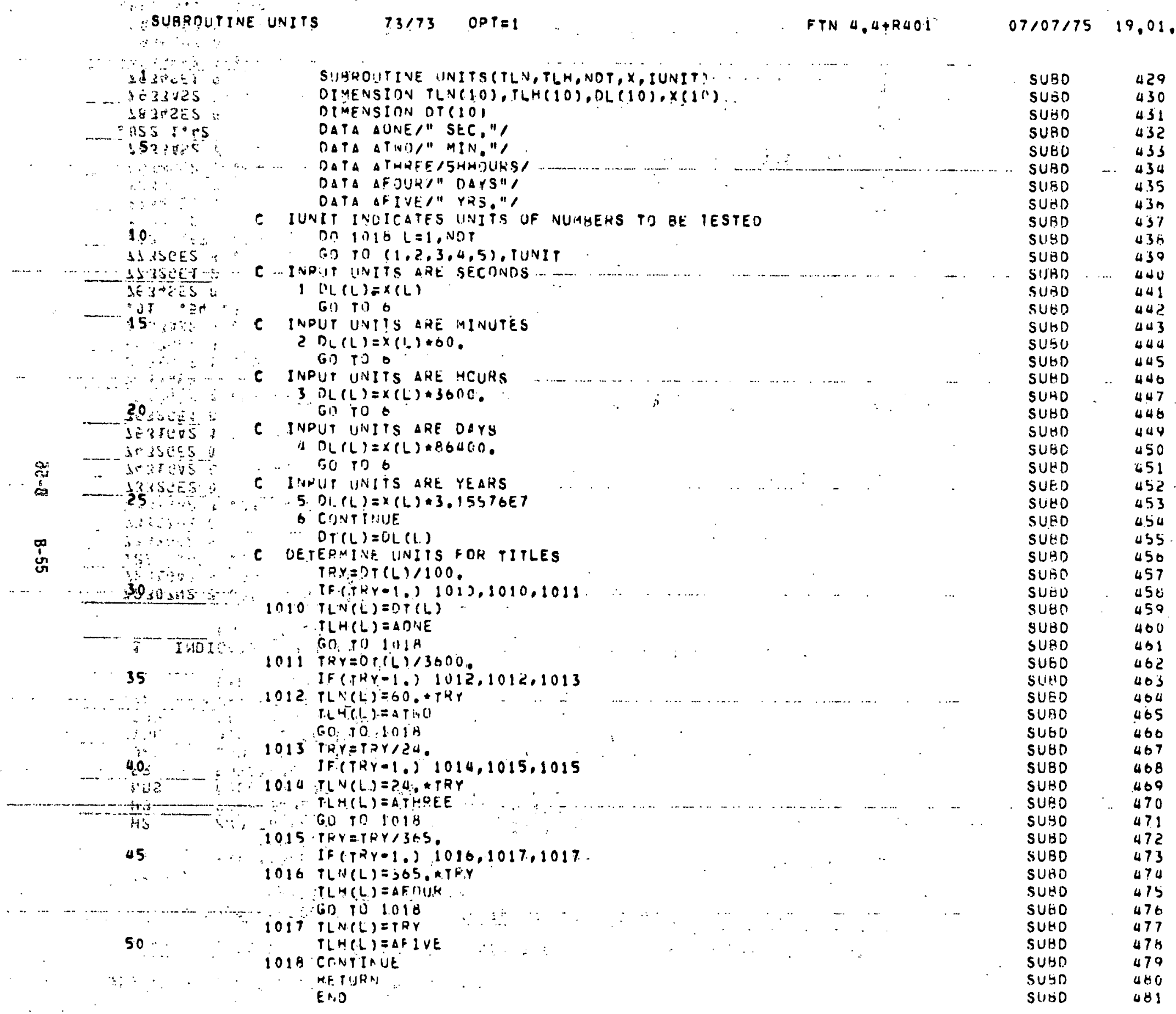




\section{FIGURE B-6. Library BISLIB Listing}

BISLIJ - GETA ENDPOINT ENERGY LIQRAQY

532500 ISOSHIELO BETA SNO POINT LIBRARY E.O. $12110, \overline{69} 19$

C

THE SYMBOLS IN COLJUAS 73-5 INOICATE THE SOURSE OF THE DATA ONA FAPTEYULLO ISOTOFE.

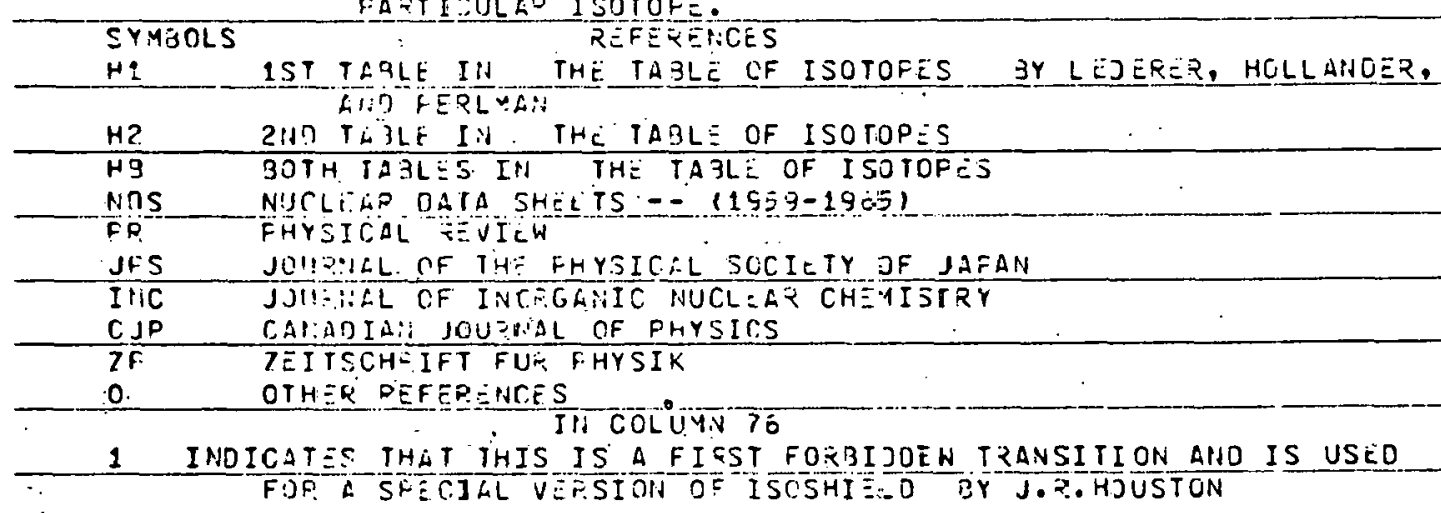

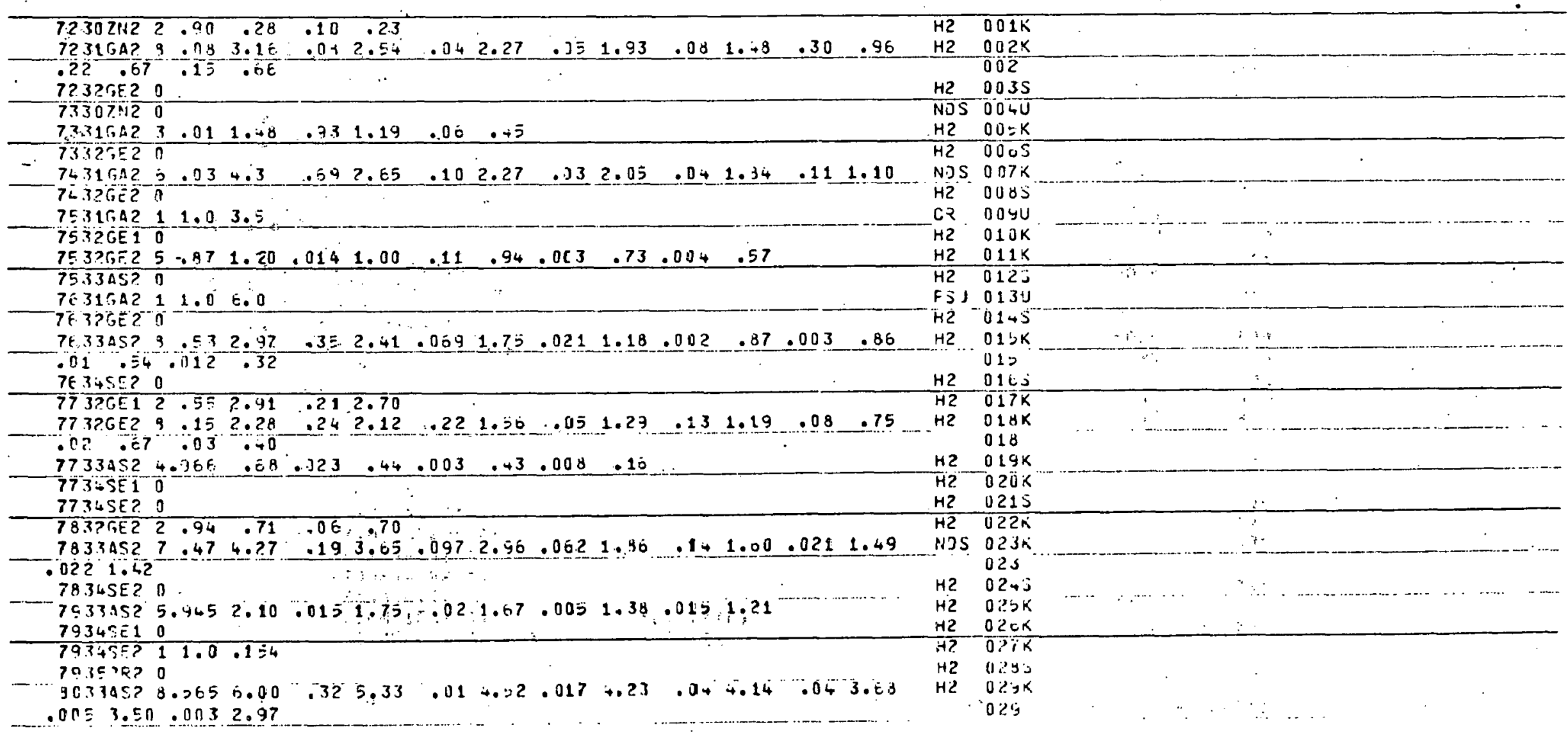

$.0 n \equiv 3.50 .0032 .97$ 
3ISTI3- उETA EMBFCI:AT ENEQGY LITRAEY

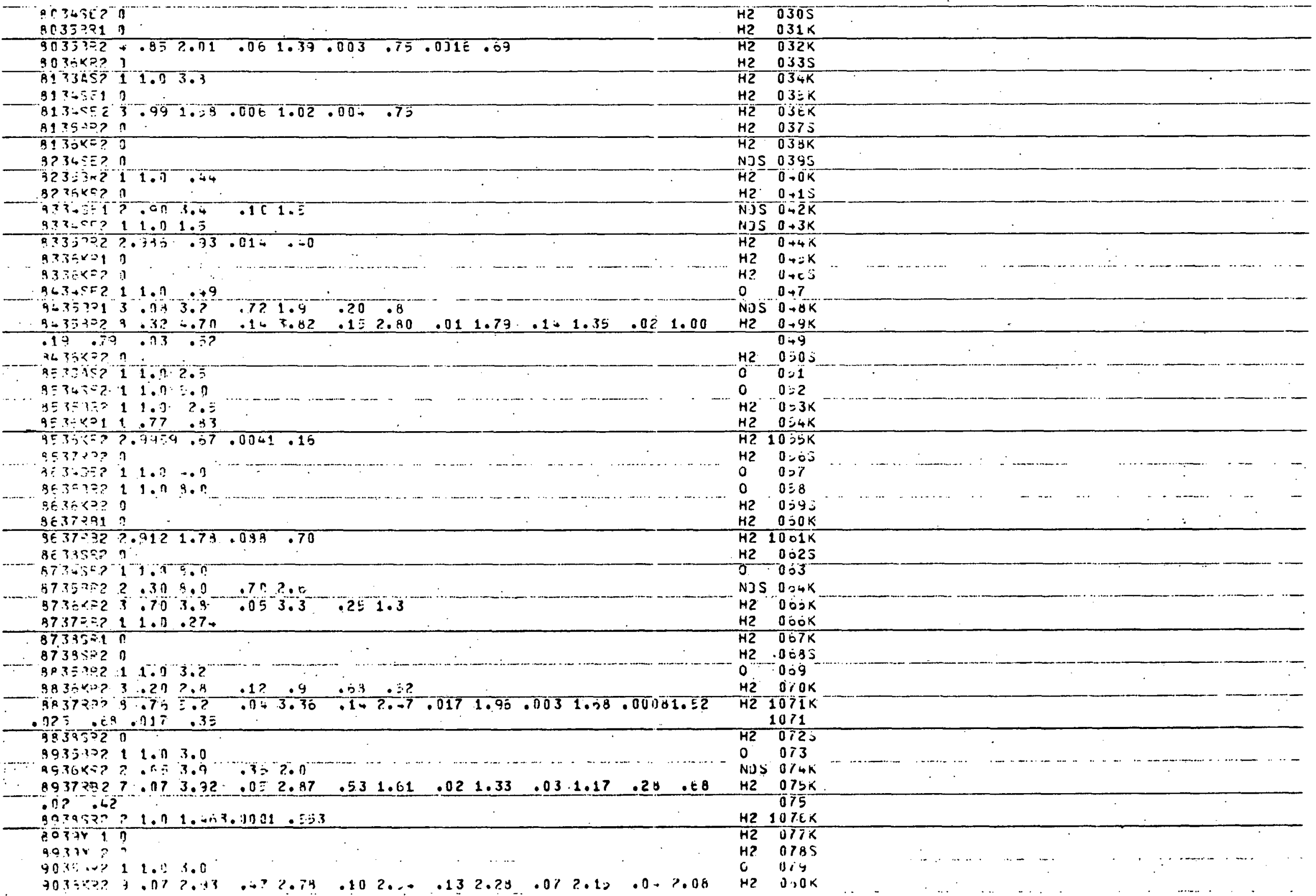




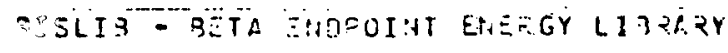

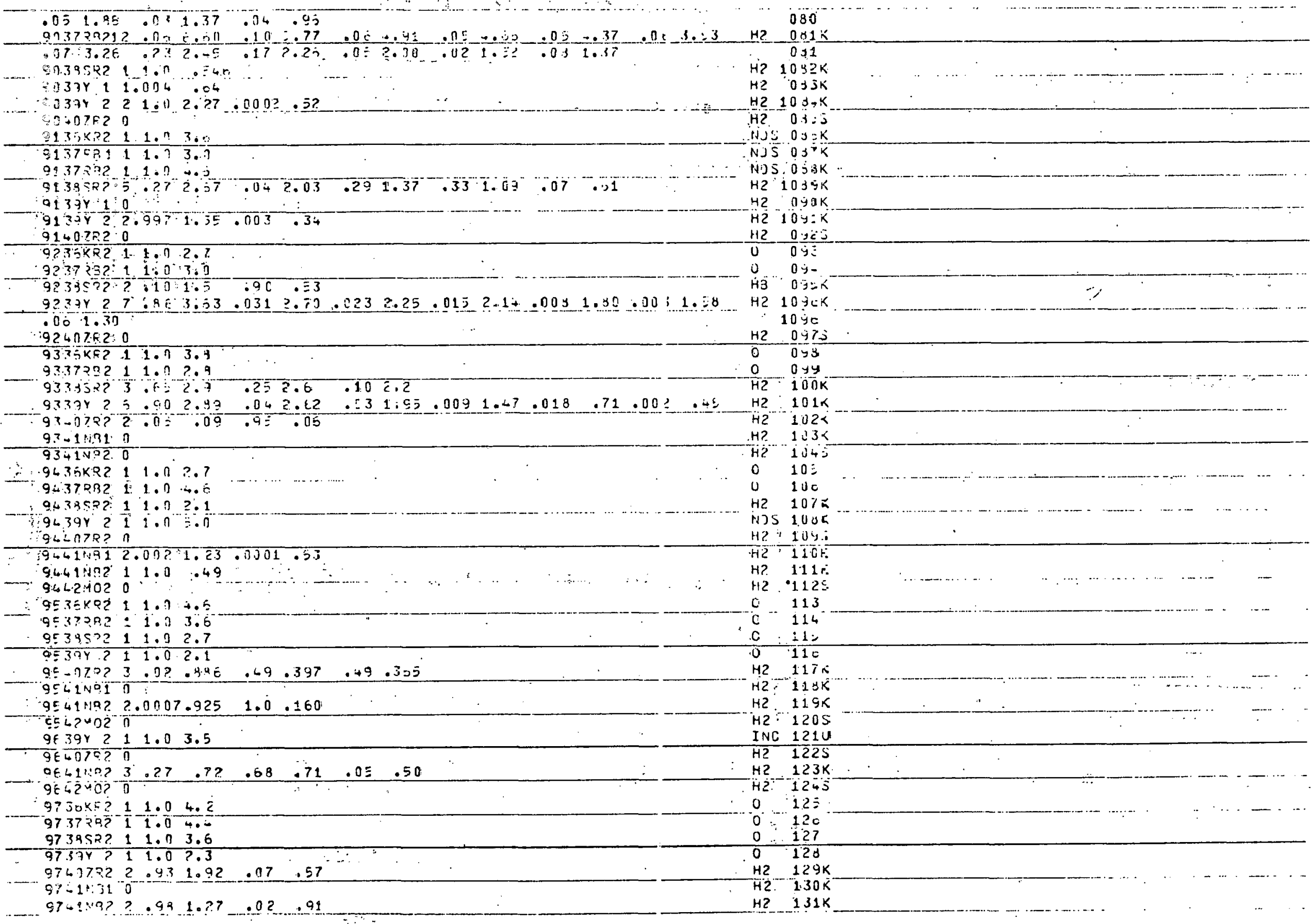




\section{ISTID= JTA ENTGOINT NNGGY LITKATY}

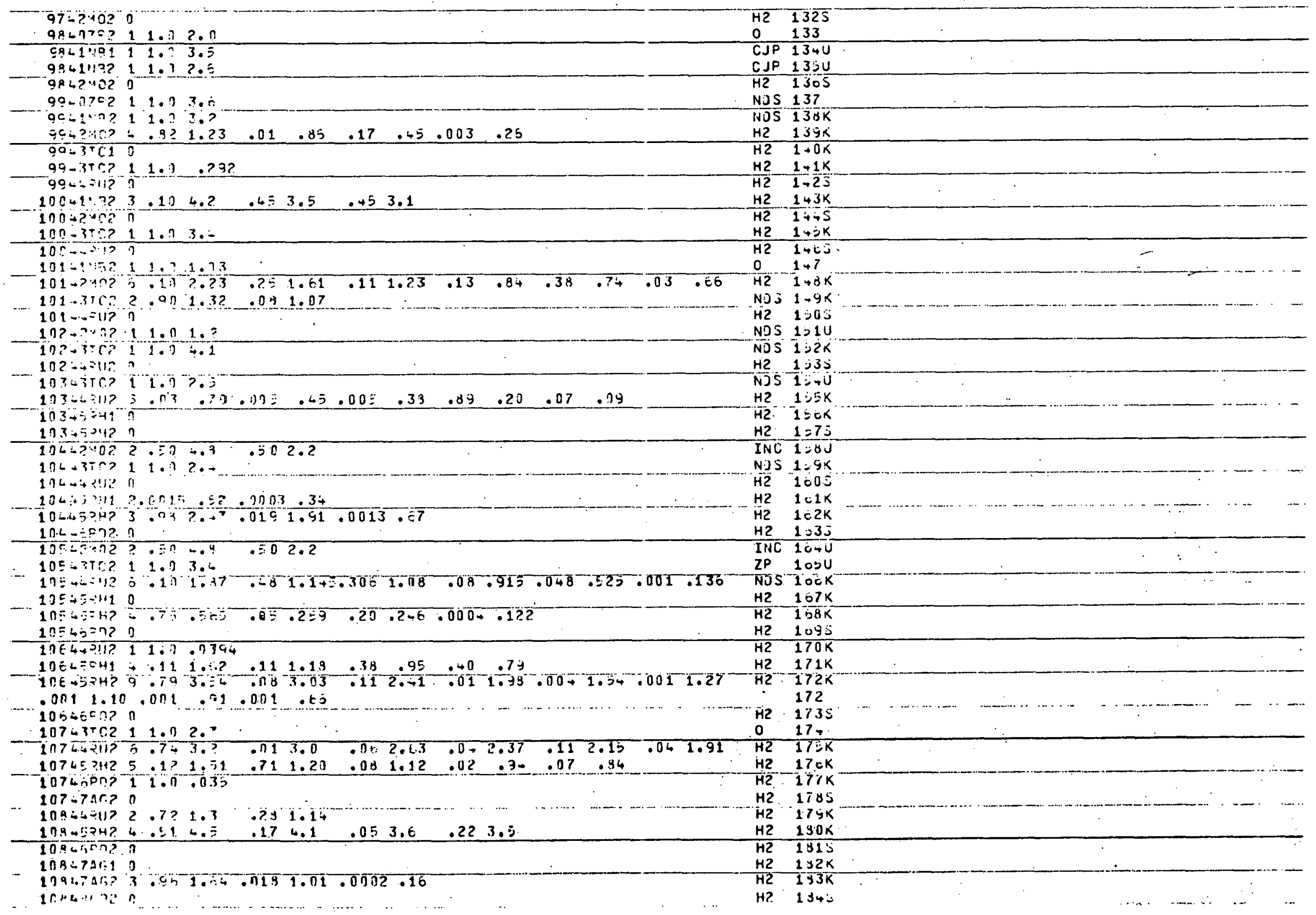




\section{BISLIS - 9ETA ENDFOINT ENEYGY LIARARY}

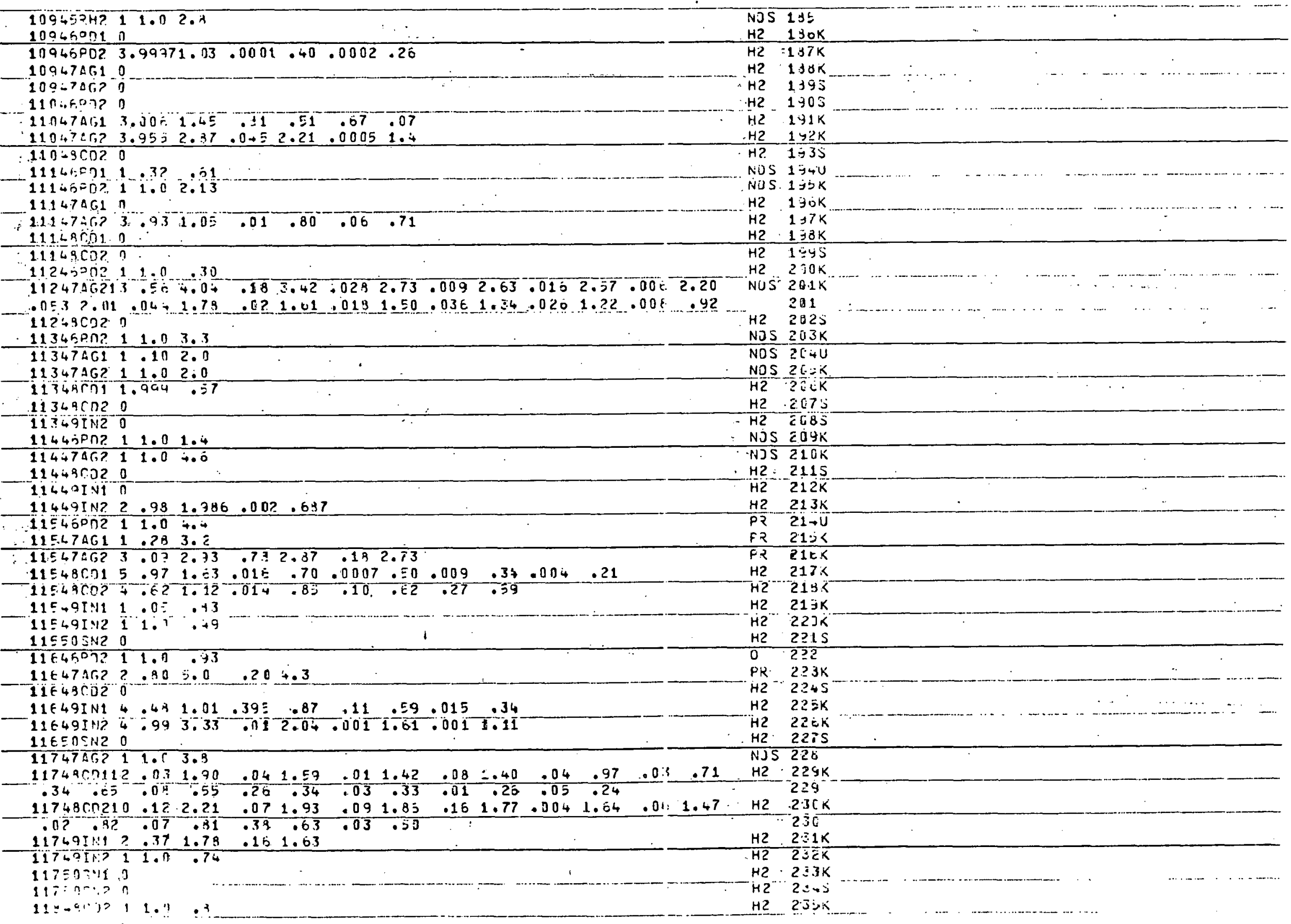

$117: n-2, n$

$11:-4.10121 .1$ 


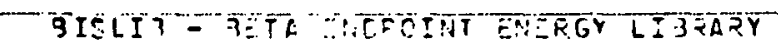

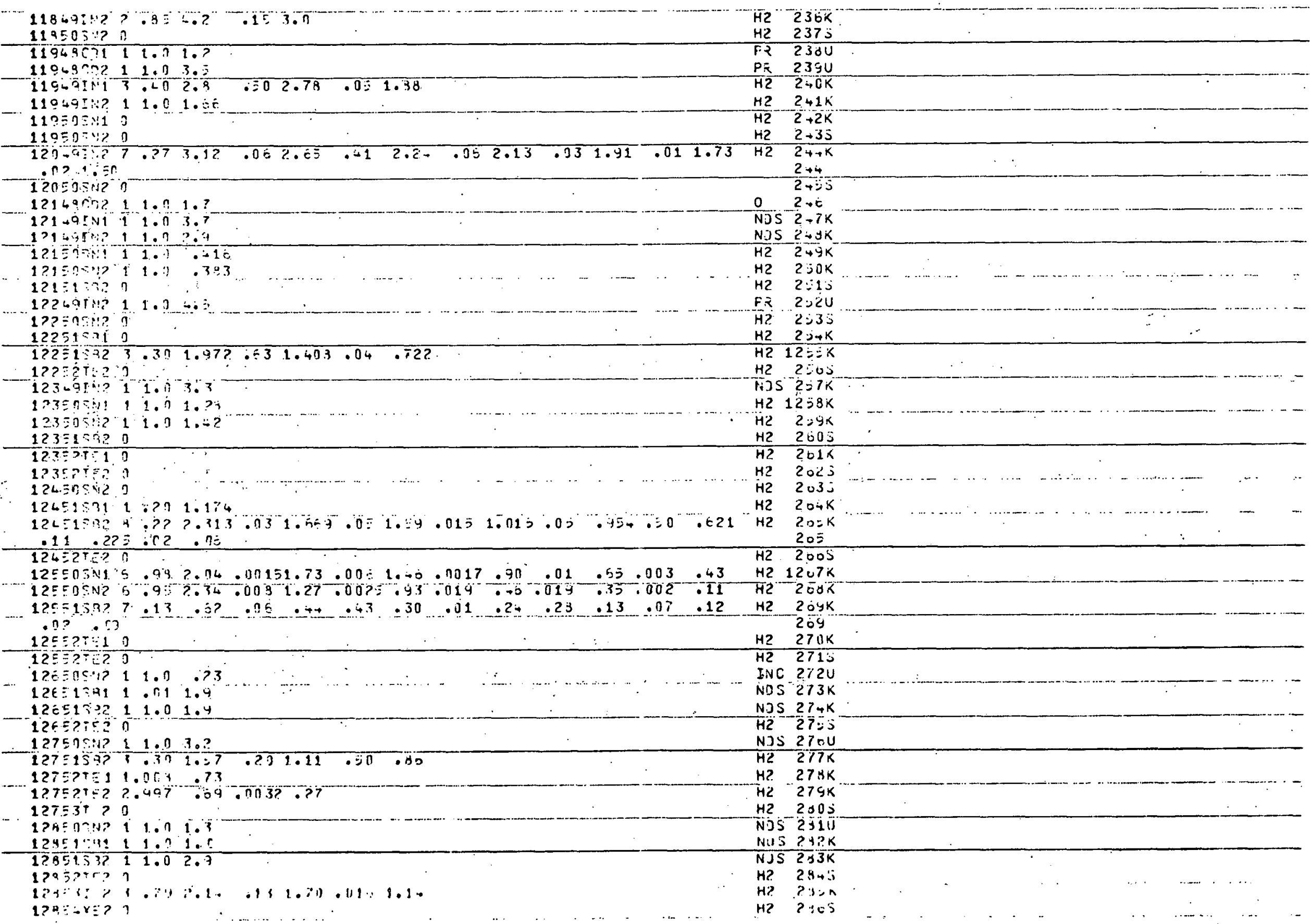


9ISLIT - 3ETA ËNDPOIN- ENEPGY LIBRAFY

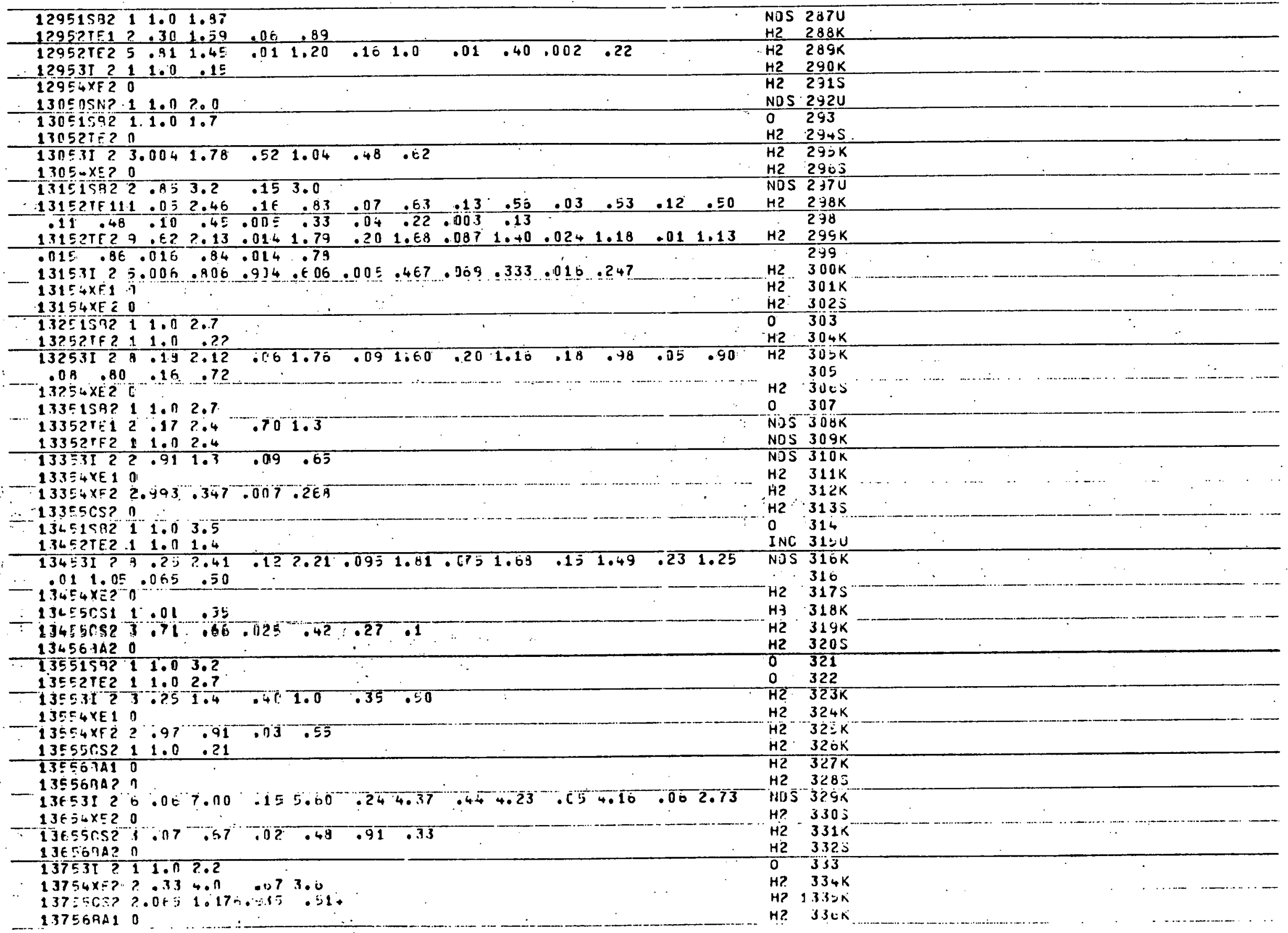




\section{BISLIQ - RETA ENDFOINT ENERGY LIGRARYY}

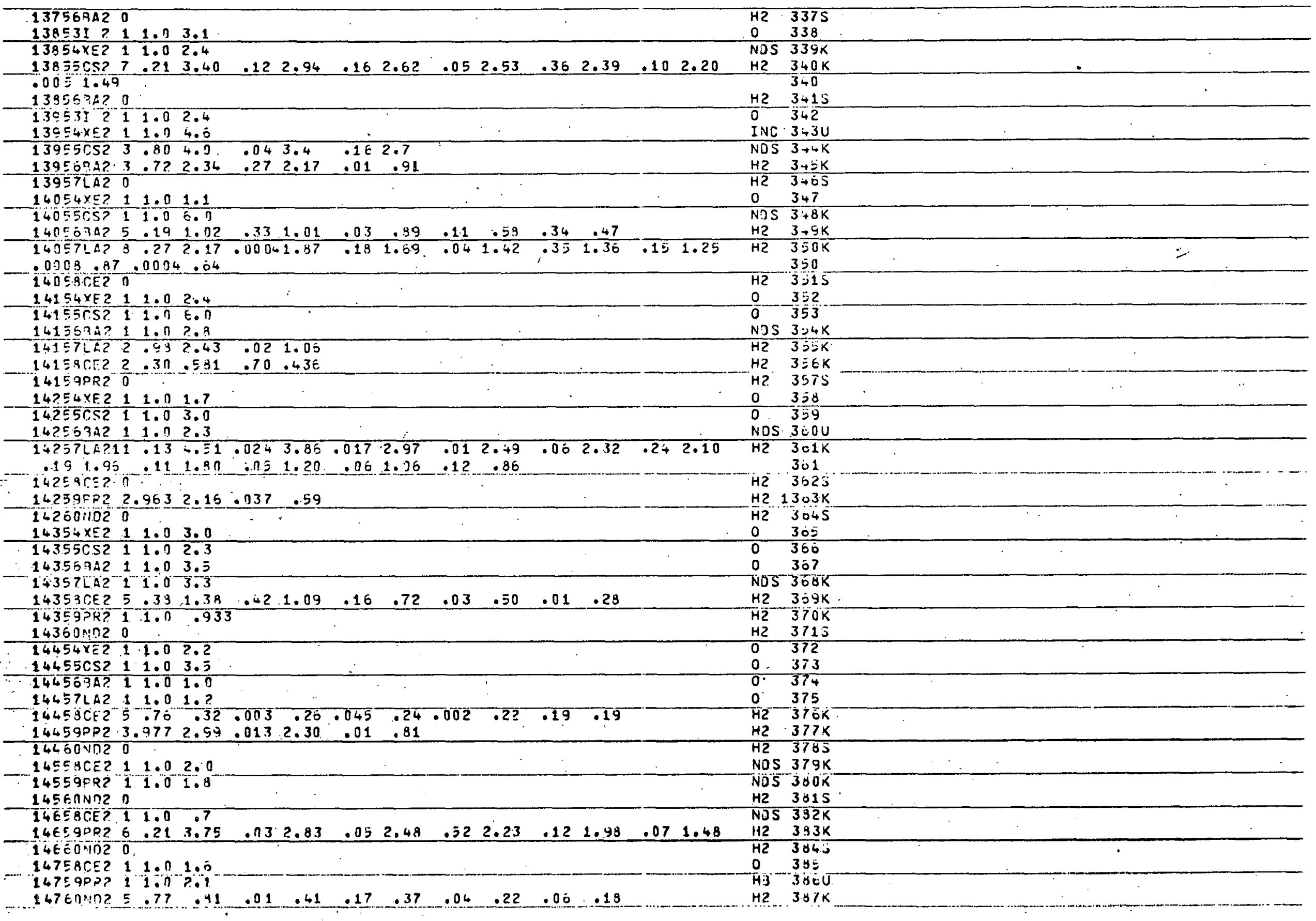




\section{BISLI क - BETA ONJPOINT ENEFGY LI BRARTY}

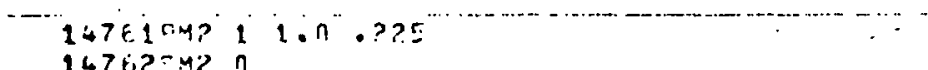

$1485.8 C . C^{2} 11.01 .1$

$1495900211.04,2$

- $1486040 \% 0$

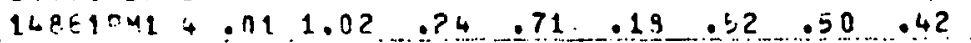

$-14: E 1<\cdot 23: \equiv 02,48$

149f?SM? ?

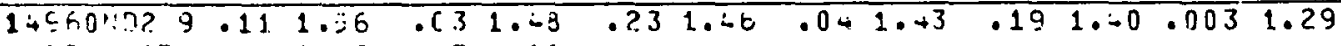

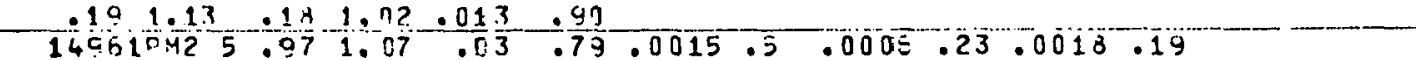

$1496 ? 5 M 20$

15060.1020

150 FुMA?

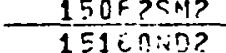

1516 กir?? 11.02 .0

$1516192.9 .10 .1 .19 \quad .061 .13 \quad .111 .05 \quad .03 \quad .95 \quad .43 \quad .84 \quad .10 \quad .73$ $.05 .50 \cdot 11 \cdot 35$

151E?SM? 2.983:076.017.055

- $15163 \equiv 020$

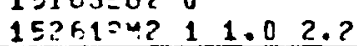

$15 ? E 25 Y ?$ ?

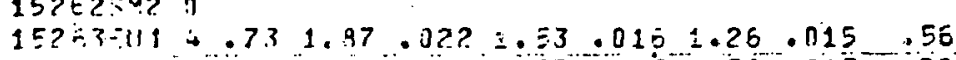

$152 f ? \leq 420.091 .48 .0151007 \cdot 15 \cdot 70 \cdot 017 \cdot 20$

$152 E \div 50 ? 3$

क $15361 \% 121.01 .5$

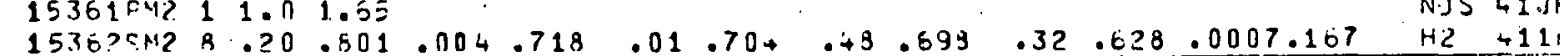

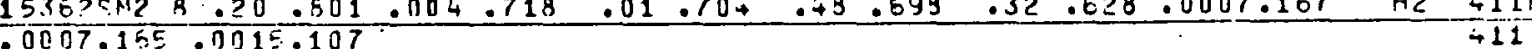

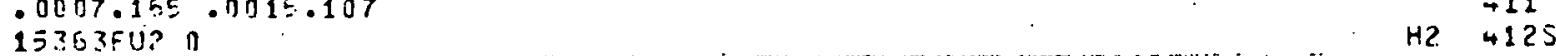

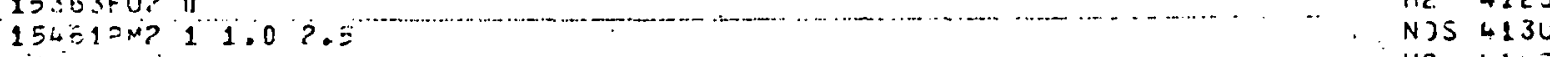

$1 \equiv 46 D 5 \div 20$

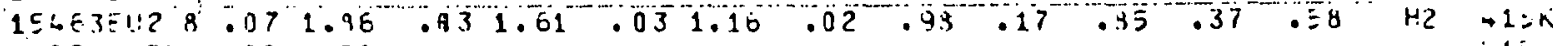

$.0 ? .30 \quad .29 \quad .26 \quad$ _

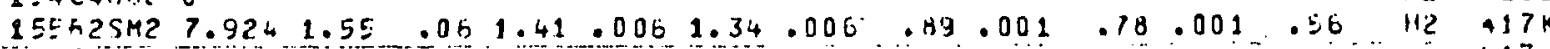

$\because 002^{\cdots} .39$

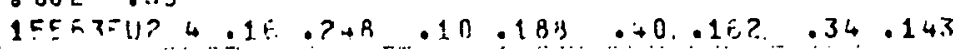

$1 \equiv 50 \operatorname{sin?} \theta$

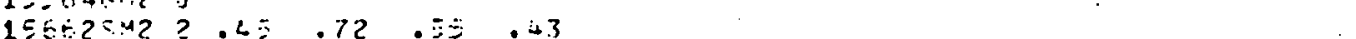

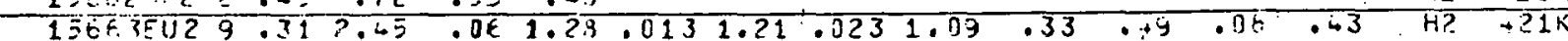

$\begin{array}{llllllllll}.022 & .27 & .09 & .20 & 0.08 & .23 & 421\end{array}$

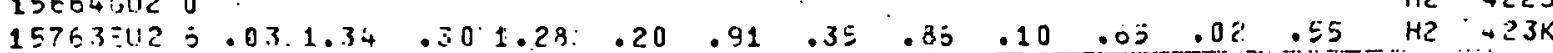

$1 \equiv 7 \equiv .0020 \quad \div \quad-32 \rightarrow 2+5$

$15863=U 211.02 .65 \quad$ N.JS +2EU

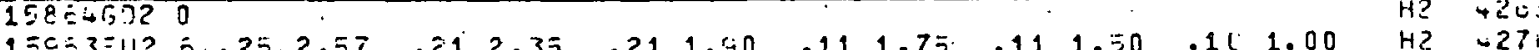

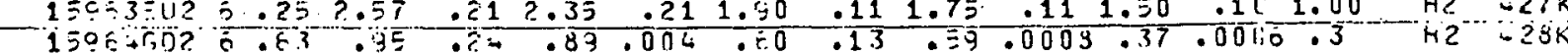

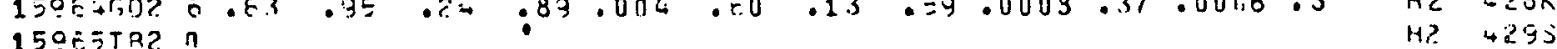

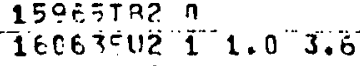

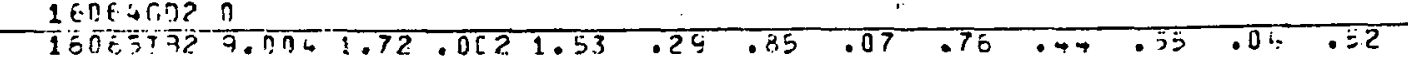

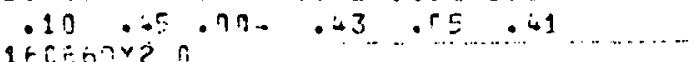

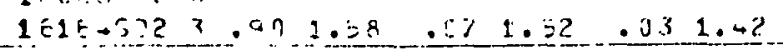




\section{BISLIG - 3ETA ENOAPOINT ENEF.GY LIBRATY}

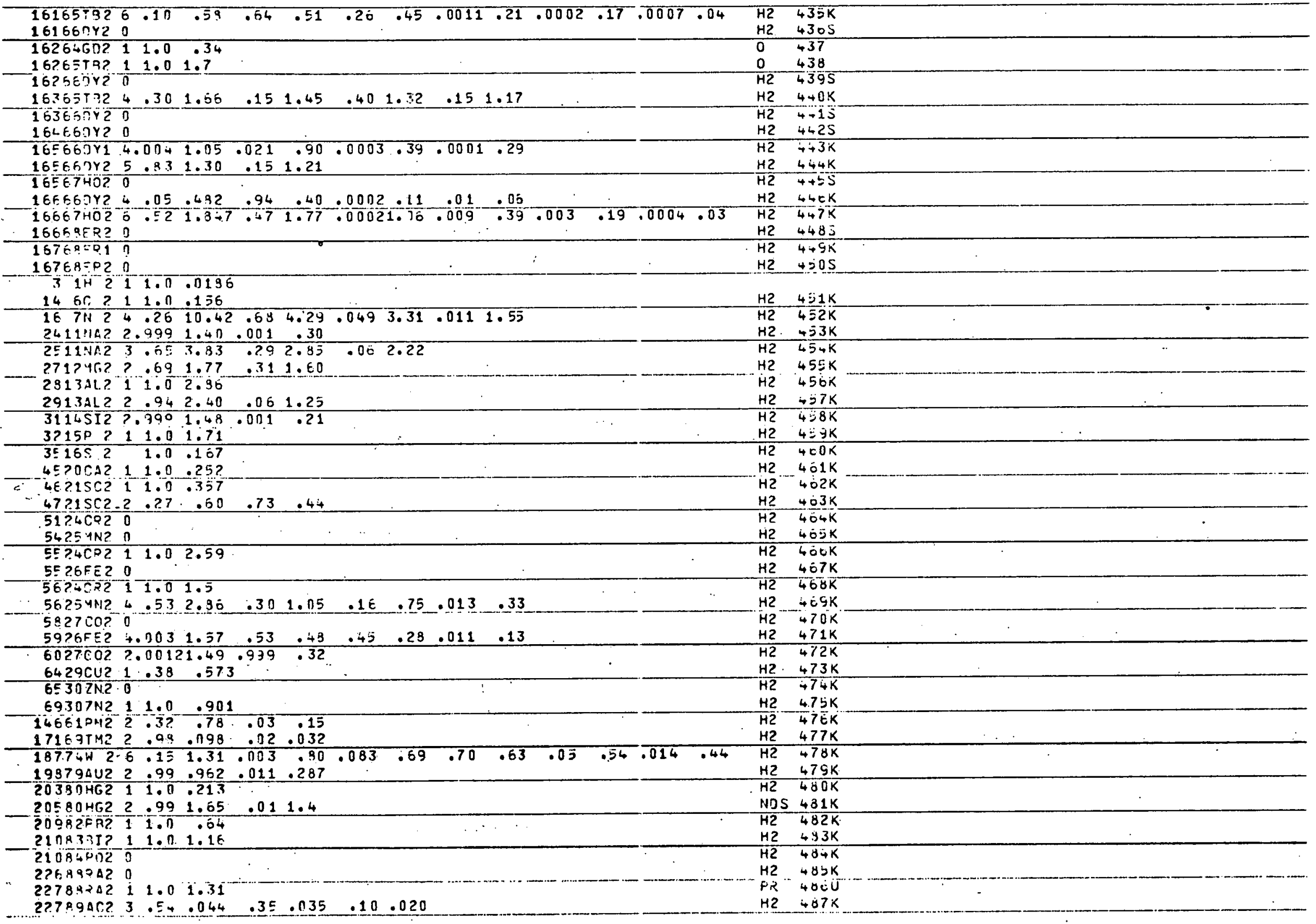


3ISLI3 - 3ETA EIOPOINT EVERGY LIBRARY

22B83 $04211.0 \cdot 0 \equiv$

2330 THH? 1 . 97 1.24

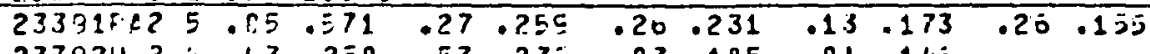

23797112.433 .250

$2399.5 \cup 20$

23994 PU2 0

$2403 \div+1120$

$2413 \rightarrow F U ?$

2419544 ? 0

$2429 \div 112$ ?

24295241

$242.754: 42 \quad 2.34 \quad .66 \quad .50 \quad .62$

24496042
1.3 .021

$$
+2
$$

$\mathrm{H}_{2} 48 \mathrm{BK}$

$\mathrm{H2} \quad 439 \mathrm{U}$

H2 $440 K$

$H 2-11 K$

$H 2 \rightarrow 42 K$

$H 2493 K$

H2 $454 \mathrm{~K}$

$H 2$
$H 2$

$H_{2}+4 j K$

He $4 . J 7 K$

$\mathrm{HL} .48 \mathrm{~K}$

$\mathrm{H2} 499 \mathrm{~K}$

H2 -500 


\section{FIGURE B-7. Library RNDBET Listing}

331 COMBINEO DATA UIHRARY THERMg SAVELIES O5/23/75

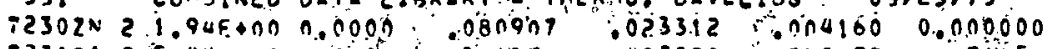

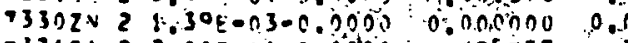

73310 a 200E-01-n

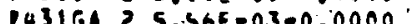

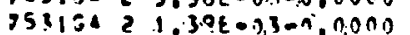

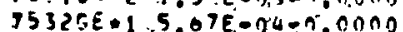

4257.35

1.019124
522075

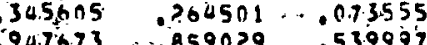

$452340 \quad 1.367110 .001540$

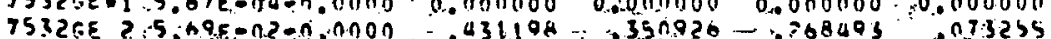

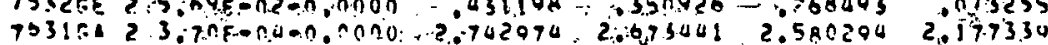

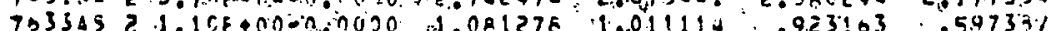

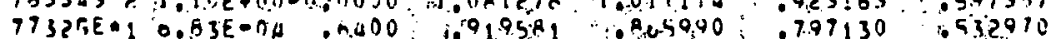

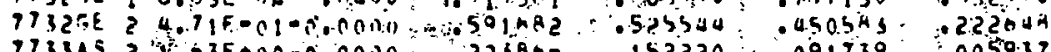

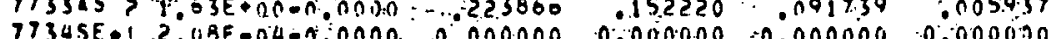

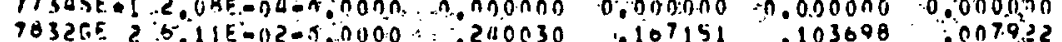

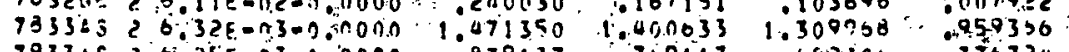

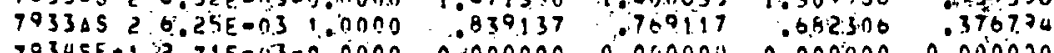

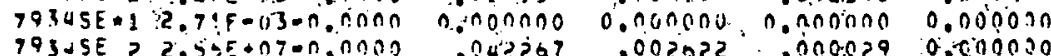

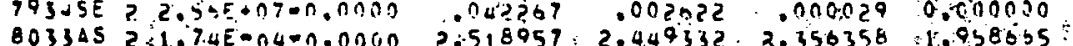

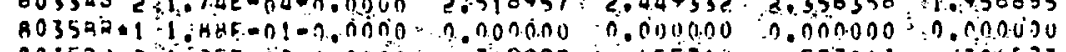

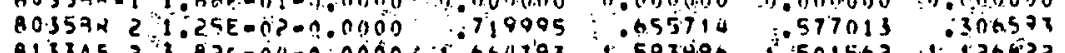

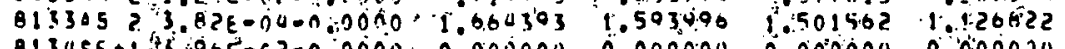

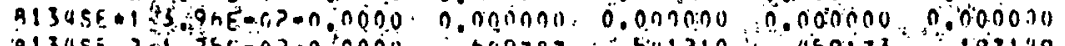

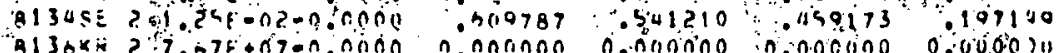

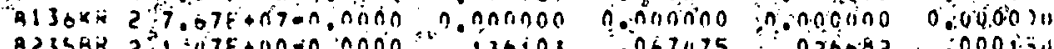

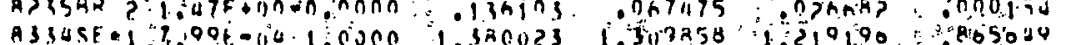

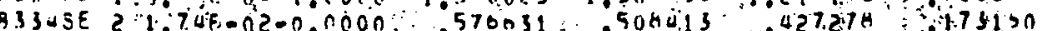

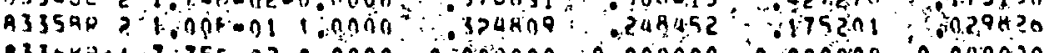

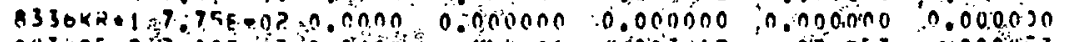

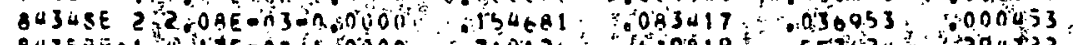

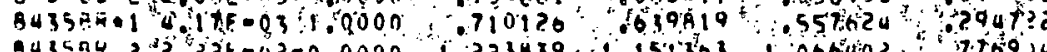

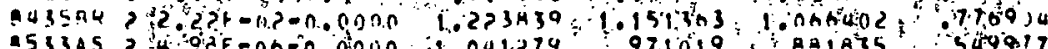

55334 ?

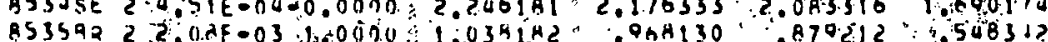

B5304201

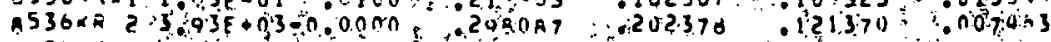

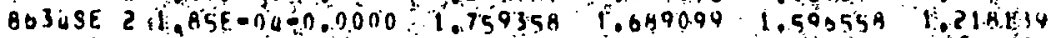

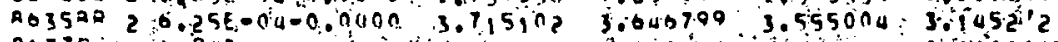

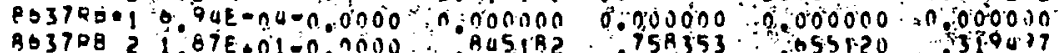

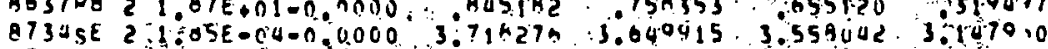

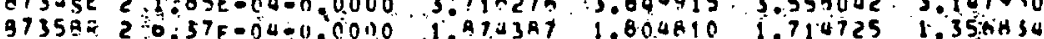

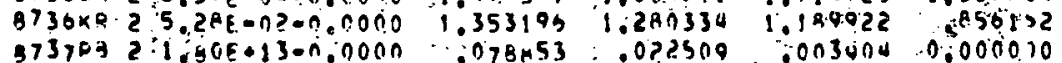

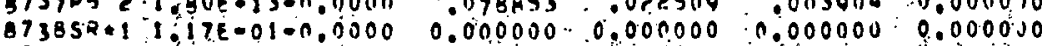

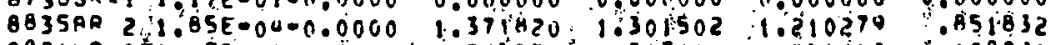

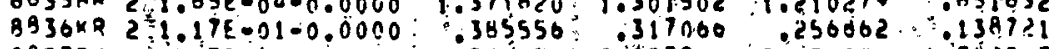

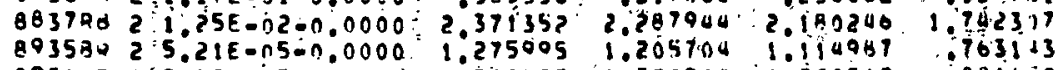

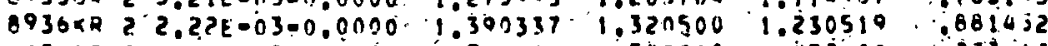

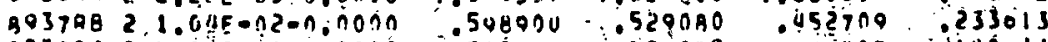

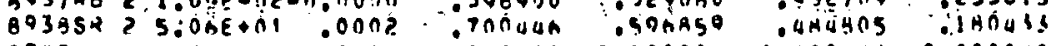

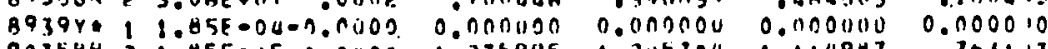

$90354421.45 E-05-n$.nIOn 1.?75045 1.2USina 1.114047 .105113

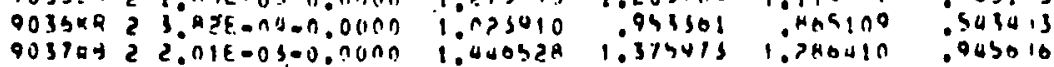

\section{(n)}




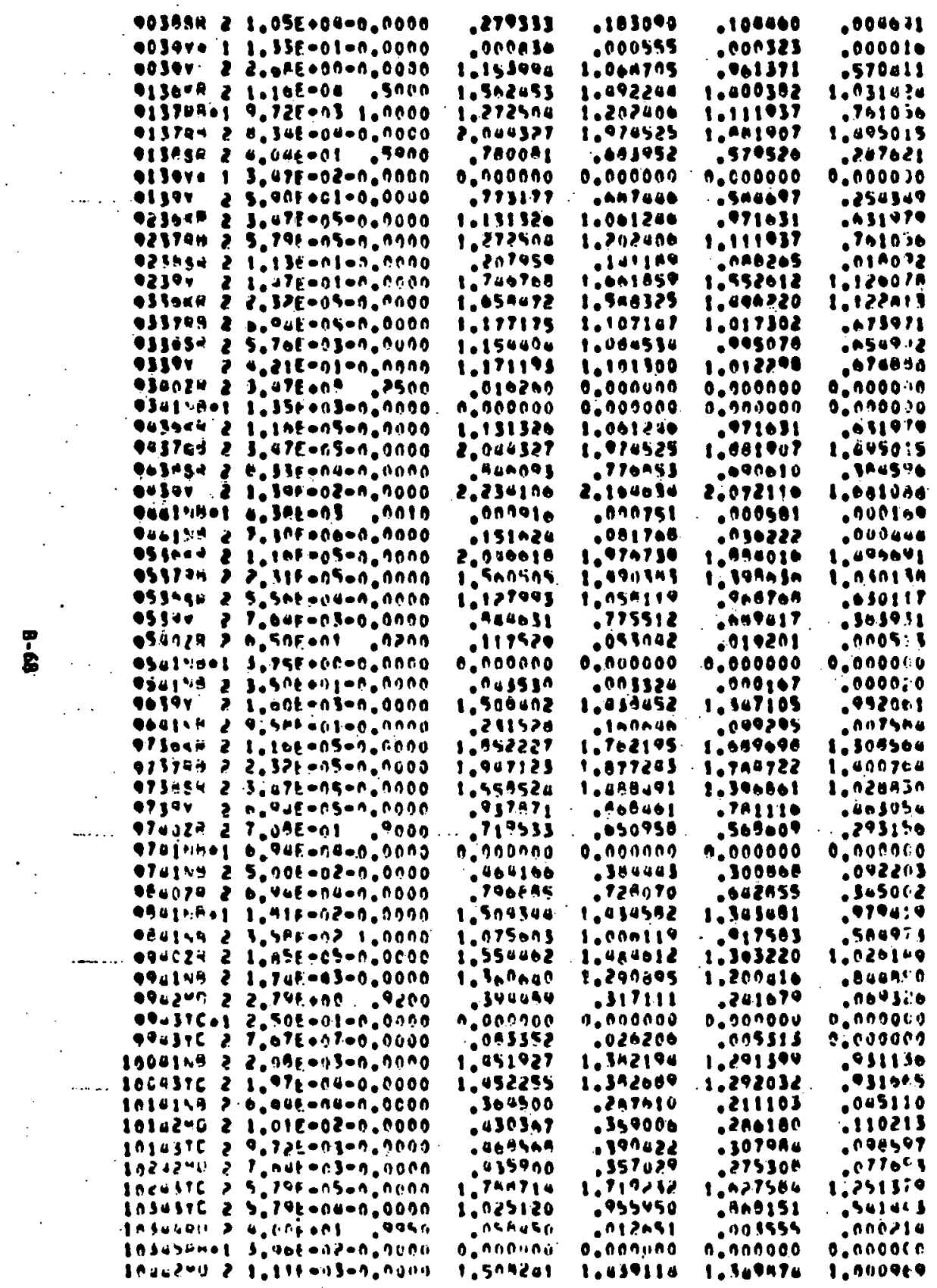


$10403: C 21.25 E-0200.0000$ $10405 \mathrm{EM}+1.008-03.0013$

1054240 z $403+-0400.0000$ $10542402.4 .631-04-n .0000$
$105035 T^{2} 5.568-03-0.0000$ 1050375 2 $5.568-03=0.0000$
$105440421.85 F-01,0600$

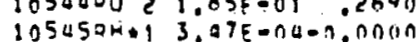
105452N? 1,50t+00 0,nr,On logusay z 3 . 5500 a n onno 100158m+1 Q. ITE-n? 1.000n 107ubic 2 s 1070 व $107452 \mathrm{H} ? 1.51 E-012-0.0000$

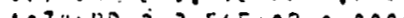
$3.135-03-0.0000$ II $305 \%$ a 2 . Ingug 0 . 1084

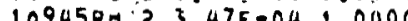
$1094000.13,335=03000000$ $1092000 ? 5035=01,0000$

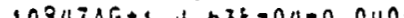

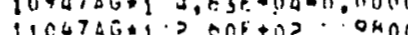

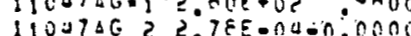
$1114000+12,29 k-111^{\circ} 3200$ $111600021.53 E=02,0000$

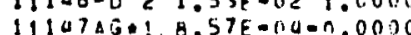

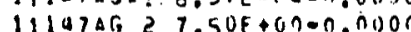
III 4 AE $1124+30$ 2 9 75E-n1-0.0000 ii $2+7102133 E-01-0.0000$ IIjuopu 1 bus

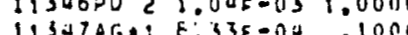
$11347 \$ 6 ? 2,211=01-0.0000$ iijuardoei $5,11 E+1,3$. $1140000<1007 t=0500.0000$ $11447 A G$ Z 5.79E-n5-0.00NO $114491 \mathrm{~N}=1500 \mathrm{E}+01-0.0000$ $114001 N 2$ B $33 E-04-0$ nnOn $115000025021 E-C 4$ 2मेC $11547 \Delta G+1 ? .5 ? 6=0141,0000$ $11547 A G 21.50 E=02.0400$ $1154 E C 0.1$ JOE +01 1.0000 $11548 C D=2.30 E+001.2000$ 11549 IVA1 $1.53 \mathrm{E}=01$.055 $115491 \mathrm{~V} 22.208+1700.0000$ $116000023.478-0000.0000$ $110478621.7 a k+n 3=0.0000$ l1609ival 3.75E-102 !.000

….... 11649IV $21.62 k-04-0.0000$ $174850.11 .33 E-C 11.0000$ 1174800 ? $1.00 \mathrm{~B}=\mathrm{al}$ 1.0000 $117491 N=1$ T.92E-C2 $11709 ! \mathrm{N} 23.13 t-12-n .0000$

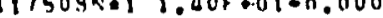
$11504(1), 3.47 E-n 2-n$. तIOn

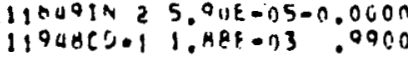

.009184

.035050 . .000077

1.508241

1.505251

.420300

0.000000

$1.430114 \quad 1.349874$

$1.382690 \quad 1.292032$

0. nnaso n.onanoo

-0h7rbl

0.0001000

0.000000

10778

34 Ho48

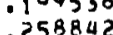

1.119294

49954

0.901395

1.173075

1.094567

0.0700000

$0.01) 7000.0 .00000$

- 717103 1.551919

0.0000000 .0000000 .000000

.05087 .039445 .460602.

$1.162823 \quad 1.093048 \quad 1.000900$

$0.00 \cos 0.00110000 .0 .000000$

$0.0000 n a 0.0000000 .000000$

0.000000

0.000000

1.016797
.020778

.000219

061त71

.000219
.77448

0.000000

0 innoino

348340

i). non 000 (1)

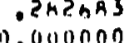

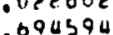

0.0001000

.207574

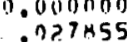

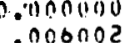

$1.301200 \cdot 1.271758$

1.349120

1.325870

1.235034

0.063476
.718904

.111290 .0157712

177378

518500

2.019605

0.0000 .00

1.950735

0.000000

.352520
1.850535

0.000000
.613702

.69585

$1.7652 \% 0$

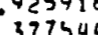

.353192

.33304 .6

1. 125072

.537506

.1152251

$.0 n 423$

.245610

.170314

.

.08000.

.035441

$.17203^{\circ}$

2.070511

$\therefore .944035$

102715
1.241800

1.331000

1.241806
1.474905

$.163372 \quad .113117$

$.403490 \quad .332 ? 20$

$.316203 \quad .273060$

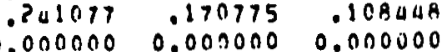

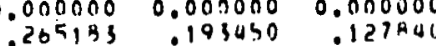

1.6baq18 1.57nz

$.352100 \quad .271511$
$.500: 09$

.090001

10001109

.931105

0.000100

00135

-50
0

$006 n 20$

0251514

701 ias

0.042 .00

n00.00

.090 .32
.208 .57

0.000 .100

.205 .62

0.000100

$.0446 i 54$
.000100

.001 is

. B 1 I IZ

.000134

$390: 111$
000.100

.04510

0.1900100

.00010

.वट7

- मीकटाइ!

. त34.756

. not 170

.

- a

.327050

1,345509

. 23436

- 6 tos.spo

03970.0

.000570

- Docisa

.020435

- 00500

886020

.100318

.031379

.129591.

.009609

0.000000

ivsato

.0100? 


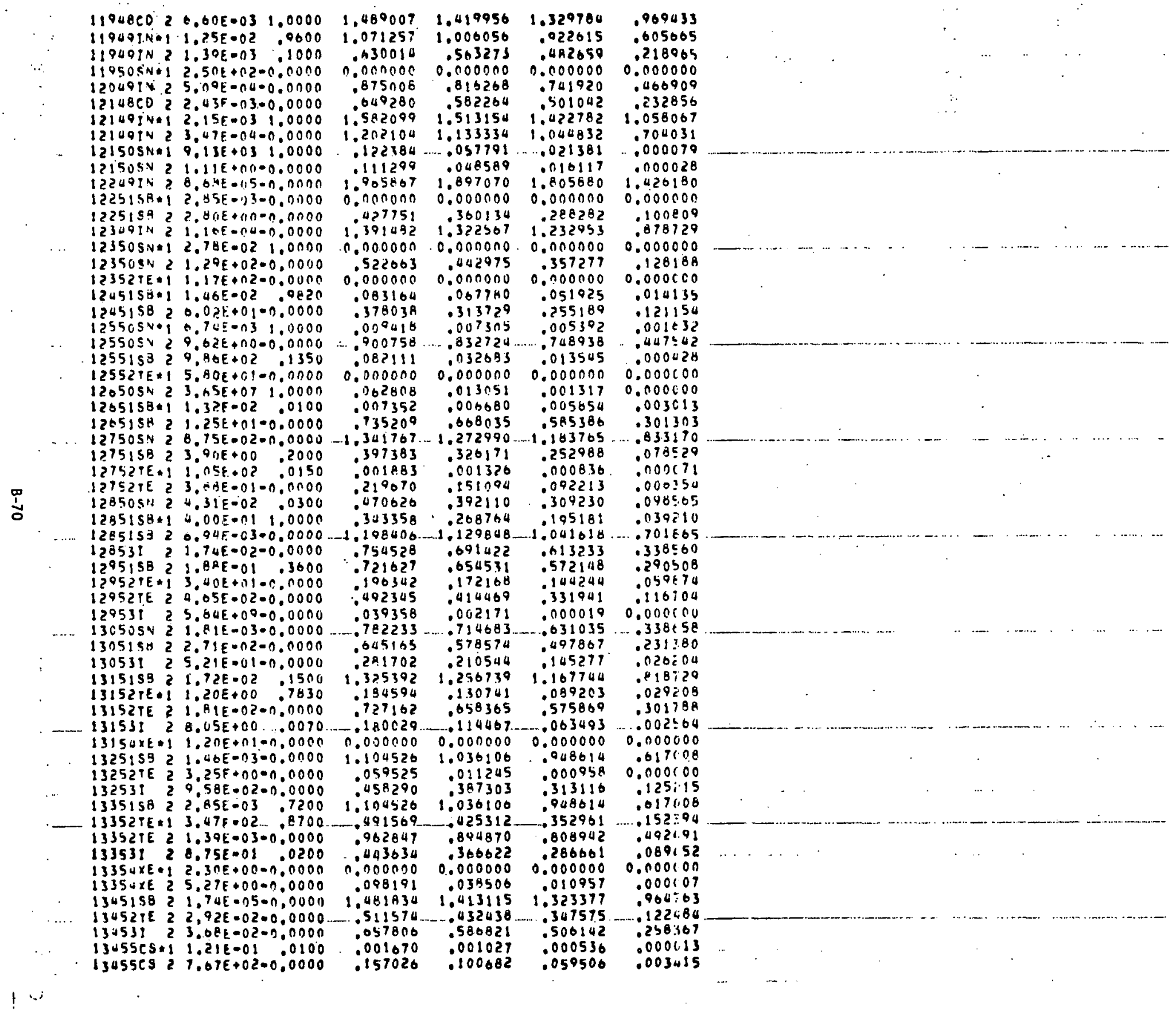


$13551592 \cdot 2.205-05-0.0000$ $13552 T E$ Z 3.30E=0J-n.01100 135531 a $2.70 \mathrm{~F}=01 \quad .7700$ $13554 \times E=1 \cdot 1.11 E-02-0.0002$ $13555 C 5 ? 7.30 \mathrm{E}+0 \mathrm{~B}=0.0000$ 13556RA+1 $1.51 E+00-n, n n O N$ i $30531 ; 901 E-c j-n$ OnCO

-1305SCs. 1 1.30E+n1-n nono 137531 z $2.78 E-n 4=n . n 0 u 0$ 1375 SYE E $2.02=-0.3-n, 0000$ $13755 C 5$ \& $1.10 E+04.200$ 1375baA+1 1.AIE-03-n.Gnn IउEs3I 27.2 ar=05-n.000

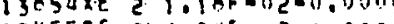

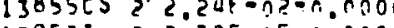
13953 ? $2.356-05=0.0000$

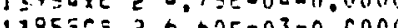
13955C5 2 0.the-03-0.rinno

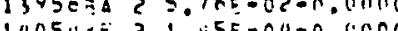

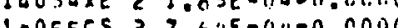
1.055CS 2 ?. SUE

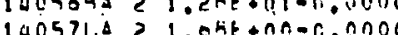

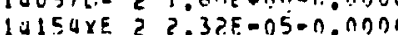

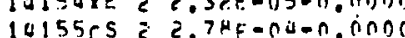

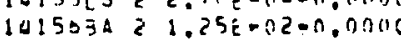
1a157La z i.nze-cilen.non

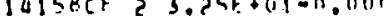

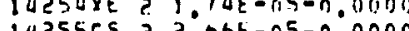
$142.55 C 5$ ? $2.06 t-05-n .0000$

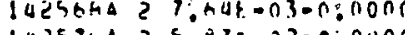
14257 La? $5.235=020-n$ inuon $10259=2 ? 2.005-0,1=0,000 n$

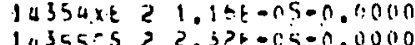
$143555522.526-c 5-0.0000$
14350942

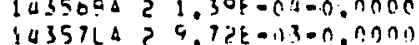

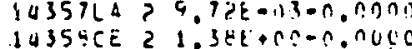
$14354 C E 2$
1435002 1405UAE 2 1. 16E-05-n.0000 14us5es 22,3 ? $5=05-n .0000$ lujsoa ?

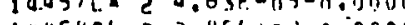
1445 "Ct $22.85 t+02-0.0000$

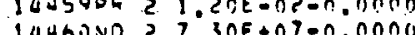
14558CE? 2.1)RE-n3-0.nUnO 14558CE ?.2.1)RE=n300.nONn 1465 CE ? $14058 C E$ ? $9.726-030-100000$ $1475 E C E$ ? $9.04 E-04-n .0000$

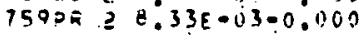
1ajooNo.? $1.11 E+01-0.0000$ 14701EN $29.49 E+02-0.0000$ 1476>54 $24.40 F+1\}-6$ non 14 $1435009 \geq 1.30 \mathrm{t}-113=0.00 n \pi$

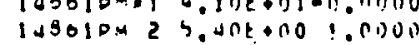
$1.330480 \quad 1.270828$ -3inata 0.0000000 .05615 ?

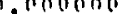
$1.075>07$ 1.

1.504901 0.503307

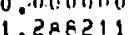
.0500720
.05858 1.096750 0000 ? 1.965764

1.599655

- 00352

- Solyss

2.

- Truna - $9590 \mathrm{rib}$ ?.67001 $1.1407>0$ . 040052 .101047 $\because 640917$ 1.235577
909220 008337 .007271 1.235867 -911112 1.407676 1.372705
.357002
.30903 - 300503 . 34.703 h $1.4723 \mathrm{nB}$ .338034 - 20 का $\therefore 00184$ $1.20 \mathrm{sga}$ - 76007 .760176 -n>77o .220255 - s40090 - Ir - ?24066 oncononan $375(12 a$
1.702500 1.792560
1.44377 - 701000

$.03421^{\circ}$ .245347 $.0070 n$ .02795

0.000000

.907004

- 801207.

.510508

- 120133 . obrnoo .210707 .091370
1.029965 .893136 1,531458 .833002 2.060271 .104412 .529127 2.61)กटं 71 $1.07290 \mathrm{~B}$ - Axita>3 - 1179113

1.108458 . A41030 .749282 - 005925 1.170622 084303 1.30471 $1.36,4718$ .312901 .799037 $.4040 ? 0$ . .340511 0.0259 0.140 .05 .181755 .040887

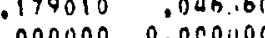
$.000000 \quad 0.0001100$ .0006690 .0001100 .000000 0.000000 . .16riso 1.4491 .10 $.710091 \quad 000580$ 1.0204201 .004470 .05alla .000ura 0.000000 0.000nolo 1.13756a - 905794 idahse

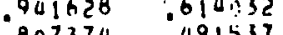
- A3 1.4399641 .461095 .

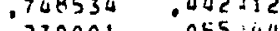
- गjonar instiva .

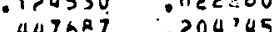
00578 . 20405

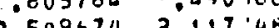
. 509570 5. . .035314 .1000064 .0905म8 .2?9.is 7iviar o. $003263 \quad 000: 15$ $1.042545 \quad .140130$ $.758329 \quad .440 .942$ 1.316313 . Hoti70 15704य 028455 IbTau 1.314 PAB 958,00 .192000 .058705 .265655 .070100 $.006050 \quad 000101$ C.000000 0.000 ico . 000380 0.33140 - henzro $\quad 3.3570$ .531701 .261.100 . 4a75za .145?5s कnstre . - 001090 o.

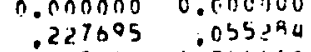
.227605
$1.035200,1.2060110$ . nus54a .002368 
$100000: 0 \geq 7.21 E-02-6.0020$ $109610 \mathrm{~m}$ 2 $2.215+00-0.0000$ $15160 \times 0$ 2 $8.33 E-33-0.0000$ 151610M z $1.175490-0,000 n$ $1516255^{\circ}$ s. $20 E+00-n .0 n 00$ $152 \mathrm{AlOM} ? \mathrm{~A}, 17 \mathrm{~F}-13-0.0 \mathrm{BOC}$ $152635 \mathrm{~V}=1$ 3.PRE-01 1.0000 $15263 E v 24.58 \mathrm{~F}+n 3-n .0 n n s$ $153012423.02 E-03=0.0000$ 153isSM? $1.9 \mathrm{AF}+0 n-n . n n 00$ $150010421.74 F=0300.0000$

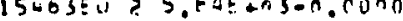
153625" $21.53 E-r: 20 n .0000$

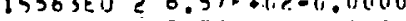

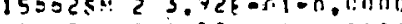
$15003 E$ ? I. S2F+r.1-n.01901) $1576350 ?+.33 t=11-0.0110 n$

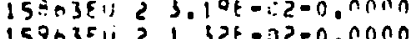

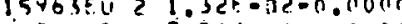
150 asto $27.5 n k-21 \cdot n, n 1100$ locosfu $21.745-\pi ?=n .00 n$ loibjit? $7.2 n F+21-n .0 n n o$ inlouran? 2. $575-0.3-n, 0 n n n$ $161651520.006+0.1)=n$. n1100 162 - $5=$ z $3.05 k+02-n \cdot 01100$ $162051725.33 k-02-n .000 n$ 163651 ? 2.111-01-0.000n $16500 r y+1.936=04$.n30n

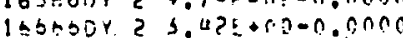
$1 b=97-0 ? 1,135+a-n .0000$ $1675250=1$ 2. $506=03-0.0000$

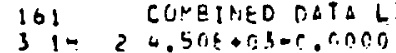

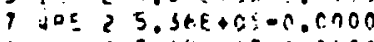
10 HAt $2 \& .18 E+D E-0 . n U C D$ is or? 2,002+00-C.0000 18 op $29.83+6 \cdot 2=n . n 000$

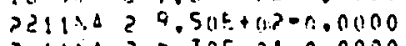
$2+1114$ द $0,30 E-01=n, 0000$ 3lust $210105001-0.1000$ उड़ा50 $21.036+01-n, 0000$ 3slas $2=.71 E+01-n .00 n 0$

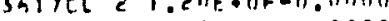
$3917 \mathrm{CL}$ ? ?.0nK-0=-0.0000 alister? परीवर ? 5 ,

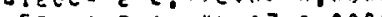

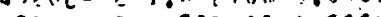
U7? $472196 ? 3.43 E+0000.0000$ $4521 S C 21.83 E+00=0.0090$ 4. $23 \mathrm{~V} 21.61 \mathrm{k}+01-n .0000$

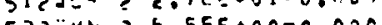

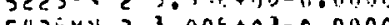

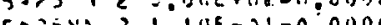
$5=205 t, 1,1 N E+03=0.0100$ $502052,1.515001=0.0000$ .353137 .705483 . ?.7?050 .012123 .534034 .047005 .000475 .219710 . 517035 - 5 tivas i - lonous - sziopo .701860 ? $1.40+57 !$ .105090 $.5 n 4748$ .145718 .093384 .624109 . 503917 .11215? - 563478

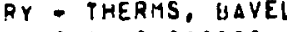
0.0 cceno 0.000 co .050277 0.000000 $0.0 n 0000$ .501306 0.000000 0.000000 0.0rosou cinnoceo - joma .070374 000000 a .112653

\subsection{0} 0.000000 0.000000 0.300000

0.000000 .1 ? 2138 $0.0 n \pi B n 0 \quad 0.000000$ . 0.000000 $0 . n n 0000$

.304785 .212209 .01752 .100907 0.100000 - 7 cajar .420978 .050000 .405436 - 03515 .839068 - Togran - Jupsty .000574 instos - 201507 -147ry . 013150 .010270 . 152567 $1.07870^{\circ}$ . 00075 . Dumpras . (01) .352310 - 352310 - Oncsiona - istaza . $52067 a$ .000000 .064106 0.00000 0.200004
.320013 .411102 .538029 - 520010 0.000000000000 0.000000 0.000000
0.000000 $0.00 n 000$ $0.045079 \quad 013637$ $087416 \quad 040337$ $0.000000 \quad 0.100000$ - nosono D.ncinnoo 0.000000 0.000000 $.003000 \quad 0.000000$ 0.0000000 .000000 .056439 0.0000na o.000000 $.1028^{\prime} 3$

0484150

. 3314139

000

$0.0,000 ; 0$

.005410

- 111530

- 10323

on 7100

.523315

$0.520 ! 0$

.0000

.003030

$.1630 \pi$

-

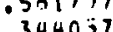

- 30 रि

od 7970

000303

. 183000 nonat 00005 (132809 11000BC 0 a $0.0 n 000$

0.000000 .0000 .00 0.00770 - Docona 0.000000 -155150 .251503 $0.0200 j 0$ 0.000000 $0.00 n 00 n$
noooco aconocos $\because$ nonono - nopona .000012 0.000000 $0.1100 c 00$ 0.00 nco 0 o. onocon $0.006 C 00$
0.000000 0.006200 .000600
.000744 


\begin{tabular}{|c|c|c|c|c|c|}
\hline & & 00 & & & 0.000000 \\
\hline $\begin{array}{ll}057<0 \\
853700\end{array}$ & $\begin{array}{l}3.8 \cap E-01-0.0000 \\
7.2 n E+01-0.0000 \\
.0 E-03-0.0000\end{array}$ & $\begin{array}{l}0.000000 \\
0.000000\end{array}$ & $\begin{array}{l}0.000000 \\
0.000000 \\
.035113\end{array}$ & $\begin{array}{r}0.000000 \\
-0,000000 \\
0.008985\end{array}$ & $\begin{array}{l}0.000<00 \\
0.000<00 \\
.000187\end{array}$ \\
\hline $\begin{array}{l}602760 \\
5923 \mathrm{N1}\end{array}$ & 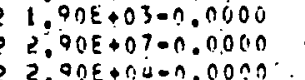 & $\begin{array}{l}.097017 \\
0,000000 \\
0,000000\end{array}$ & $\begin{array}{l}0.03010 \\
0.000600 \\
0.000000\end{array}$ & $\begin{array}{l}0 ; 000000 \\
0,000000\end{array}$ & $\begin{array}{l}0.000<00 \\
0.000<00\end{array}$ \\
\hline $\begin{array}{l}695841 \\
6558 v 1 \\
605904\end{array}$ & $\begin{array}{l}0 O E+O L-n .0000 \\
1 O E-01-0.0000 \\
3 O E-01=0.0000\end{array}$ & $\begin{array}{l}0.0000 n 0 \\
0.000000 \\
.071668\end{array}$ & $\begin{array}{l}0.000000 \\
0.000000 \\
.045098\end{array}$ & $\begin{array}{l}n, 000000 \\
0.000000 \\
0024293\end{array}$ & $\begin{array}{l}0.000<00 \\
0.000<00 \\
.000140\end{array}$ \\
\hline 65302.4 & $2.455+02-0.0 n n 0$ & 0.000000 & 0.000000 & 0.000000 & 0.000000 \\
\hline $\begin{array}{l}69397 \mathrm{~N} \\
693079\end{array}$ & $\begin{array}{l}5.60 E-01-0.0000 \\
3.60 E-02-0.0000\end{array}$ & $\begin{array}{l}0.000000 \\
.310854\end{array}$ & $\begin{array}{l}0 . \cos \pi 000 \\
.242817\end{array}$ & $\begin{array}{l}0.000000 \\
.160244\end{array}$ & $\begin{array}{r}0.000100 \\
.020 \% 09\end{array}$ \\
\hline 306 & $208+0.1=0.0000$ & 0.008 & n.ounnou & 0.000000 & $0.0001: 00$ \\
\hline 3345 & $h(1 E+01-n$. & & 0.0 & & 0.0 \\
\hline 743345 & $1.75 E+n 1-n .0 n n 0$ & $0 . n$ & 0.08 & 0.0 & 0.000 .1100 \\
\hline $753+6 E$ & i. $?$ ?tE+ns-n.,non & & 0.000000 & $n n$ & 1.00 \\
\hline & $\theta \cap 5=02-n$. & 0.0 & $\therefore \cdot n$ & 0.0 & $0.000: 60$ \\
\hline & SOE+nit-n. & 0.0 & 0.0 & & 0.0 \\
\hline (2) & $1 . \ln _{\beta}+0 n=0.0000$ & $0.00000 n$ & 0.000000 & $0.0 n$ & \\
\hline & DE & & 0.0 & 0.0 & 0. \\
\hline תודו & $3.0 E+D O=0.00000$ & 0.0 & 0.0 & 0.0 & $\begin{array}{l}0.600 ; 00 \\
0.000 ; 00\end{array}$ \\
\hline $\begin{array}{l}974390 \\
074396\end{array}$ & $\begin{array}{l}\operatorname{\theta nosin} n \\
. \cos \theta\end{array}$ & $\hat{n}, n f$ & 0.0 & $\begin{array}{l}0 . n \\
0 . n\end{array}$ & 0.000100 \\
\hline ل & $B O E+2 D=0.0000$ & 0.00 & 0.08 & 0.0 & \\
\hline $\begin{array}{l}1034600 \\
1054140\end{array}$ & 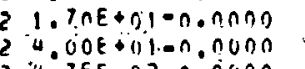 & $\begin{array}{l}0.000000 \\
0.000000\end{array}$ & $\begin{array}{l}0.0010000 \\
0.000000\end{array}$ & 0.0 & $\begin{array}{ll}0.0 \\
0.0 \\
0.0\end{array}$ \\
\hline & 100 & $3 . n$ & 0.000000 & $\begin{array}{l}0.0 \\
0.0\end{array}$ & $\begin{array}{l}0.000780 \\
0.000=00\end{array}$ \\
\hline $\begin{array}{l}113691 \mathrm{~N} \\
1135015 \mathrm{~N}\end{array}$ & $\begin{array}{l}-n .0000 \\
=1.01000\end{array}$ & $\begin{array}{l}0.000000 \\
0.000070\end{array}$ & $\begin{array}{l}0.000000 \\
0.0 n n 000\end{array}$ & & \\
\hline 125531 & o.vist +ol-n,nono. & .009243. & 0.000000 & 0.nooroo & 0.000000 \\
\hline 190 & $1-33 E+01-0 \cdot 1000$ & $\begin{array}{l}0.000 \\
0.00\end{array}$ & 0.0 & 0.0 & 0.8 \\
\hline $\begin{array}{l}13 \\
13\end{array}$ & $\begin{array}{l}: 1,0 E+h \\
: 1 O E+0\end{array}$ & & & 0.00 & $\begin{array}{l}n, 0000600 \\
0,000000\end{array}$ \\
\hline & $\begin{array}{l}1.10 E+01-0.0000 \\
2.36 E+02=0.0060\end{array}$ & $\begin{array}{l}0.00 \\
0.00\end{array}$ & 0,001 & 0.0 & $\begin{array}{l}0.000000 \\
0.000000\end{array}$ \\
\hline $\begin{array}{l}1536460 \\
1669740 .\end{array}$ & $\begin{array}{l}2.36 k+02-n, 0,060 \\
4.3+f+05=n, 00000\end{array}$ & $\begin{array}{r}0.000000 \\
.01620 ?\end{array}$ & 0.00 & 0.0 & $0 ; \cos 000$ \\
\hline & ACE+nO-n. nOnn & $0.000 n \pi 0$ & 0,000000 & 0.0000000 & $0 . n o c 0 c 0$ \\
\hline & $10 E-n 1=n$. & $0: 0$ & $\begin{array}{l}0.00 n 0000 \\
0.000000\end{array}$ & $\begin{array}{l}n .00 \\
n .00\end{array}$ & $\begin{array}{l}0.008000 \\
0.0 n c 000\end{array}$ \\
\hline $\begin{array}{l}176099 M \\
1716994\end{array}$ & $\begin{array}{l}.576+n z-1, .0000 \\
\text { out }+0 z-6,0,000\end{array}$ & $\begin{array}{l}0.000000 \\
.024001\end{array}$ & o.donono & 00000000 & 0.00000 \\
\hline i7s70 r & $4.10 E+00$ & 0.000000 & 0,000000 & 0.000000 & 0.0 \\
\hline $177716 \mathrm{U}$ & $8 \cap E+n \cap=n: 0010 n$ & 0.0 & 0.0 & & \\
\hline & $1.00 E+01-0.0 n 00$ & & 0. & $\ln$ & 0.001000 \\
\hline & & & & $\begin{array}{l}1000 \\
10000\end{array}$ & 0.0 \\
\hline & $\triangle O E+05-C$ & 100 & $\begin{array}{r}0.000000 \\
0.000000\end{array}$ & $\begin{array}{l}0.000000 \\
0.000000\end{array}$ & $\begin{array}{l}0.00 \\
0.00\end{array}$ \\
\hline & $01=n$. & & 0.0 & .103431 & 103 \\
\hline $752 \varepsilon$ & $\begin{array}{l}1.00 t+00-0.0000 \\
7.30 E+01-0.0000\end{array}$ & 0.000000 & 0.000000 & & \\
\hline & $70 E+30-n_{0} 0$ & 0.100000 & 0.0000000. & 9.000000 & 0.001 .000 \\
\hline $\begin{array}{l}\text { 157755E } \\
1897506\end{array}$ & $\begin{array}{l}1.0^{2} 05+13-0.000 n \\
7.10 E-0.1-0.0000\end{array}$ & $\begin{array}{l}0.001000 . \\
0.0000000\end{array}$ & $\begin{array}{l}0.000000 . \\
0.000000\end{array}$ & $\begin{array}{l}0.000000 \\
0.000000\end{array}$ & $\begin{array}{l}0.00: 000 \\
0.000 .000\end{array}$ \\
\hline bos. & $.5 C E+01=0.000 n$ & n. ninoono. & & 0000 & 0.001000 \\
\hline & & & & & 13000 \\
\hline & & & & & \\
\hline os & $3 O E+O D=n .0000$ & $0: 000000$ & 0.0 & 000 & $0,00: 000$ \\
\hline & $01-\cap, 0 \cap 00$ & $n .0$ & 0.0 & 0.0 & 0.001000 \\
\hline $\begin{array}{l}19.27712 \\
1907712\end{array}$ & $\begin{array}{l}\text { A5E+DI-n.nOno } \\
\text { OnE=ni-n.coso. }\end{array}$ & $\begin{array}{ll}000 \\
000\end{array}$ & $\begin{array}{l}0.000000 \\
0.000000\end{array}$ & $\begin{array}{l}0.000000 \\
0.000000\end{array}$ & $\begin{array}{l}0.00 \\
0.00\end{array}$ \\
\hline & d. & $\begin{array}{l}10 \\
10\end{array}$ & $\begin{array}{l}0.000000 \\
0.000000\end{array}$ & $\begin{array}{l}0.00 \\
0.00\end{array}$ & 0.00 \\
\hline & & $n, i$ & 00 & 0.08 & 3000 \\
\hline 1416 & & & 000000 & 0.000000 & 0.000000 \\
\hline
\end{tabular}


$107700121.50 E-01-0.0000$ 100794115 $108904 U 22.10 E+00=0.0000$ $1007041,3.158+0000.0000$. 197 BnmEt 1.00E+nC=n.0000

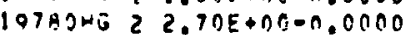

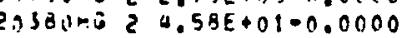
200BITL $1.13 E+00-0.0000$

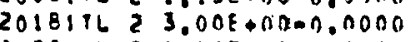
2n2911L $21.205+01-0.0000$ OO+BIIL $21.10 E+03-0.0000$ ABREA 2 ?.17E+nO-n.0000 2 ?. $17 E+n 0=n .0000 .0 .10 n 0 n 0$ a una 2008 OTBS 2079s? ?

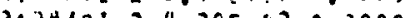

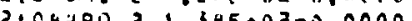
II 2) $3899 A$ 2 $1.17 E+01=n$.0nOn

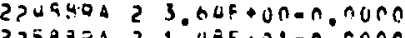

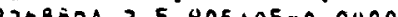
एक 2 58 तa 2 2. $53789 A C ? 8.005403-0.0000$ 2) 270014 a $10846001-000000$

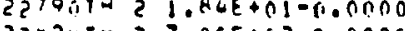
כ20901 j) 2)

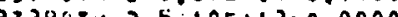
दु 3)

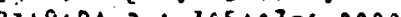
3) 23) 23. 2 द 20705004000000 350 $5000007-0.0000$ 2) द350

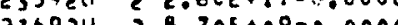
$23702020.758000=00000$

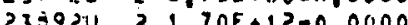

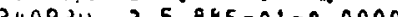
$237050=0$ ? $00 \mathrm{~B}+0 \mathrm{R}-0,0400$ $37025=0$ 2 $330+0000,0000$

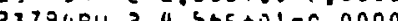
$235040423,305+04-0.0000$ 230940.4 2 $8.00 E+0 b-n, n O 0 n$ $2009+0 U 22.6(1)+06-0.0000$ $241040 U 2$ SOBNE+A3-0, तCOO

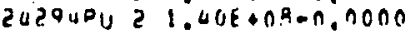

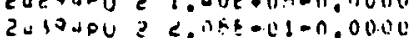
5u49upu z 2.03001000 .0000 ?.610544 ? 1.70E+na-n.nOnO

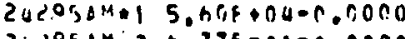

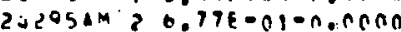
.2015505 0.000000 0.000000 0.000000
0.000000 0.000000 0.00000 a.jon gonono nongonno 0.000000
0.003005 0,000000 $.01292 \pi$ 009135 . 0000000 . 0 0 0000 . Dionon $\because 00000$ . 8.00000 , 000000 $0.0 \ln n 00$ - 000000 0.000000 0.000000 0.000000 $0.00000 n$ 0.000000 ORB $O P B$ 0000000 0.000000 0.0001000

0.000000 00000 .143070
$0.000000 \quad 0.000000 \quad 0.000003 \quad 0.000020$ r.oncoso

0.000300 $0.000000-0.000000-0.000000-0.0000000$ 0.000000 0.000000 0.000000 4.000000 $.142040 \quad .0942690 .054745 \quad .002753$ 0.0000000 .030070 0.0000000 .000030 0.0000000 .000010 0.0006800 .000000 $0.000000 \quad 0.000010$ $0.000000 \mathrm{C} \quad 0.000010$ $0.000000 \quad 0.00000 .10$ 0.0000000 .0000110

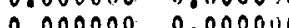
0.000000 0.0000000 .000010 $0.0614 \% 0$ $0.000000 \quad 0.000000$ $0.000000 \quad 0.000010$ $0.000000 \quad 0.0000110$ $0.200000 \quad 0.00001 .0$

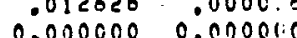
0.0000000 .01000 0.0000000 .00000 0.000000 . 0.000000 .000000 0.0000000 .000000 0.10000000000000 0.000000 0.00000 0.0000000 .000000 $0.000000 \quad 0.000000$ 0.000000 0.00000 0.0000000 .000080 0.000000 0.000060 $0.000000 \quad 0.000060$ 0.0035370 .00007 $0.0000000 .0000 C 0$ $0.000000 \quad 0.0000<0$ $\begin{array}{ll}0.000000 & 0.000060 \\ 0.000000 & 0.000060\end{array}$ 0.000000 0.n00000 0.000000 0.000000 0.00148 .90 .000000 $0.000000 \quad 0.0 n c 0 c 0$ $0.000000 \quad 0.000000$ $0.000000 \quad 0.000000$ $0.000000 \quad 0.000000$ $-0.000000 \quad 0.000000$

0.000000 .0000000

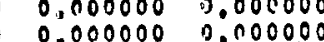

0.000000 . 0.000000
0.0000000 .000030 


\begin{tabular}{|c|c|c|c|c|c|}
\hline & co & 0.0 & 0.0 & 0.000 & 0.000100 \\
\hline & $3.21 t=$ & & 6 & 50 & 000129 \\
\hline & & .0 & 00 & no & \\
\hline & $04=0$ & & 00 & 0.0 & 300 \\
\hline & $100+030$ & & 0 & & \\
\hline & 0 & & $\begin{array}{l}n 0 \\
00\end{array}$ & & $\begin{array}{l}0 . \operatorname{nnd} 100 \\
0.000100\end{array}$ \\
\hline & $S . H \triangle F$ & & & 0.0 & 0.000100 \\
\hline & & or & noo-. & 0.00 & 0.0 \\
\hline $249979 x$ & of & 0.0 & 0. & & 10 \\
\hline 50 & 30 & n?a. & $\begin{array}{r}.170005 \\
0.000000\end{array}$ & ino & 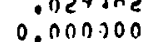 \\
\hline & $E$ & & & & \\
\hline & $n 3 k+12-$ & n nnono. & 0000 & 0.000000 & 000300 \\
\hline
\end{tabular}




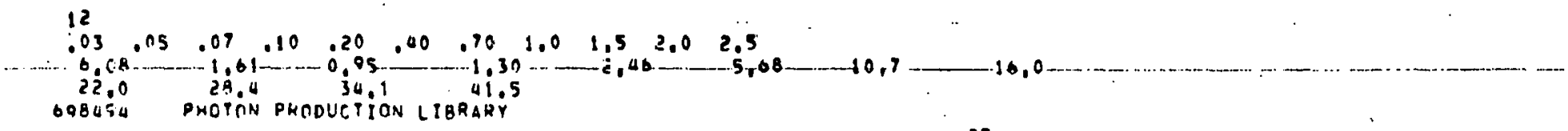

THE SYMUOLS IN COLUMNS $73-5$ INDTCATE THE SOURCE OF THE DATA ON

25 PARTICULAG ISCTCPE.

II IST TARLE IN THE TABLE OF ISOTOPES BY LEOERER, HOSLANDER,

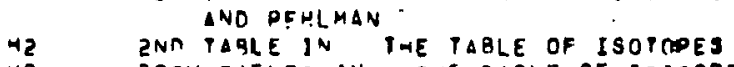

ME SOTA TAELES IN THE TABLE OF IBOTOPES

NO NIJCLEAR DATA BMEETS $=$ SECTICN $B$ (S906-1968)

NI AD ANO KI

NE. NO INO TS

O OPMER REFEEENCES

HO $M$ MI $A N O C$

OR

NO NO $A \cup D$

IN COLUMN 75 AND (2) THAT SOME DF THE INFO ON $X$-RAT(S) COMES PP. 570-1 OF

INDICATES THE STME THING

JNFO ALSO CUMES FROM PP. ETA-5 OF THE HANDBOCK OF CHEMISTAY ANO

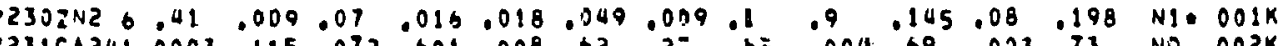

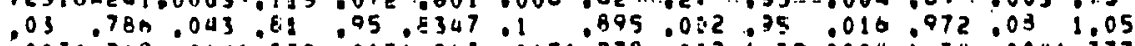
718.00071 .82 $.074 .859 .0112 .814 .00072 .15,0292.2 \quad 00332,24.0022 .49 .0122,491$ i1 $2.50^{7} .00072000 .0052 .846 .00042 .91 .0012 .976 .00013 .05 .00113 .34$ $73307 \times 2200$

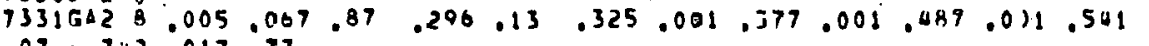

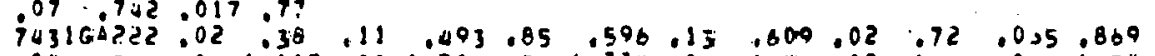

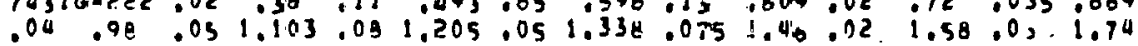
.00 $\quad 1.03 \quad .02 \quad 2.18 .49 \quad 2.35 .03 \quad 2.55 .03 \quad 2.23 .03 \quad 2.97 .0103 .17$ .0173 .33 .023 .14

$9531642 ? .01936 .03 .098$

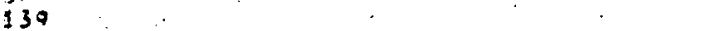
.0010 .0117

$70316423.5 \quad 55.5 \quad .563 .501 .12$

.0015

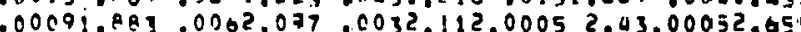

$77326=13.08,011.12 \quad 159.210215$

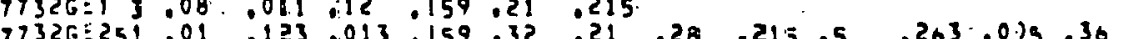

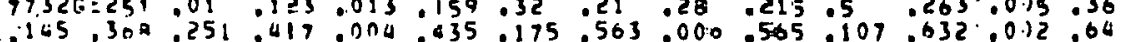

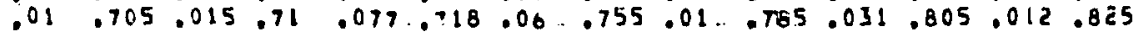

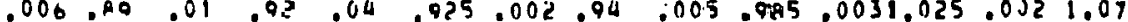
.0031 .085 .0291 .105 .0801 .245 .00501 .28 .0021 .295 .0098 .335 .0311 .37 .0121 .40 .0021 .475 .0081 .515 .0121 .50 .0031 .6 .0041 .7 .00151 .715

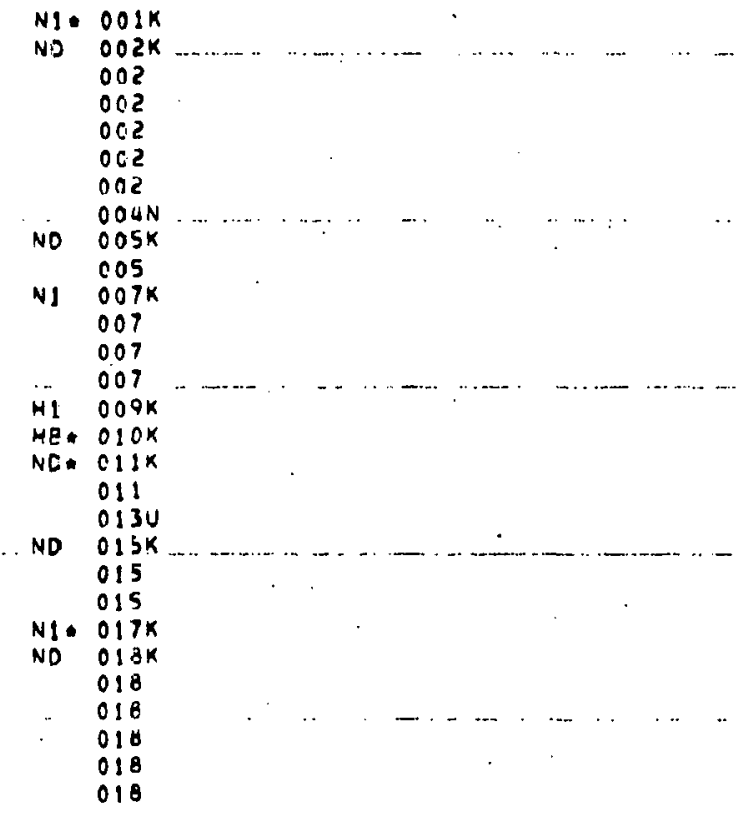


FIGURE B-8. Library GISLIB Listing

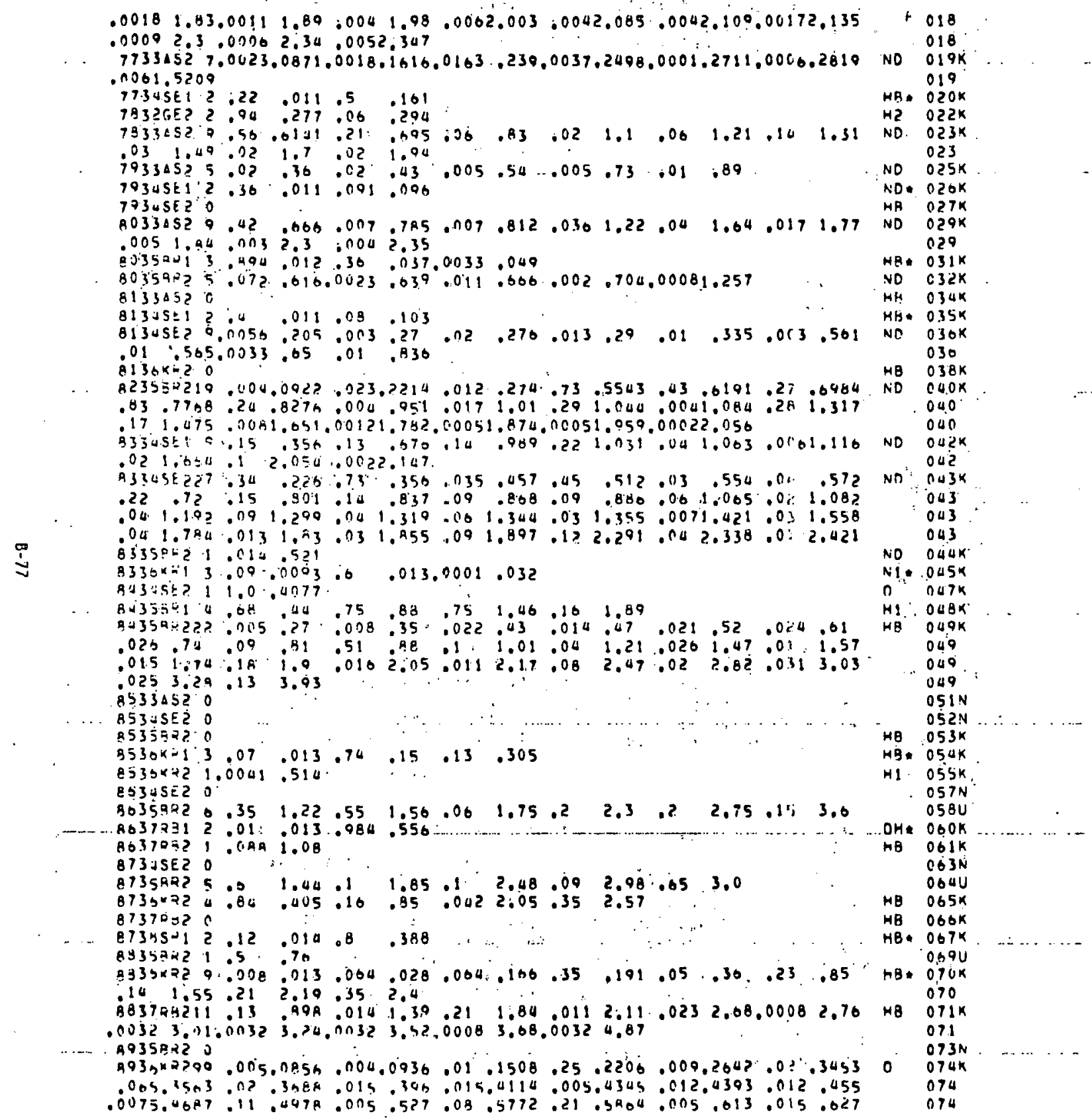


$.02 \quad .695 .009 .708 .04 .1376 .003 .744 .005 .76 \quad .006 .777 .005 \quad .002$ $.02 .023 .007 .80 \quad .00 .0075 .073 .0035 .004 .071 .005 .087 .0111 .01$ $.0091 .077 .0561 .105 .0251 .117 .0111 .173 .0031 .273 .0051 .298 .0161,320$ $.0231 .37 .0051 .472 .0041 .5 \quad 0111.533 .011 .530 .0071 .065 .011 \quad 1.07$ $.0471 .602 .03 \quad 1.74 .0281 .775 .0111 .043 .0121 .902,0041.998 .0(202.011$ .0172 .012 .012 .12 .022 .241 .0042 .38 .0142 .6100082 .644 .0072 .753

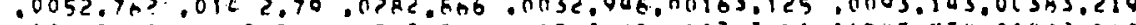

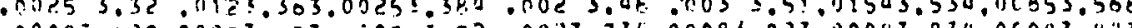

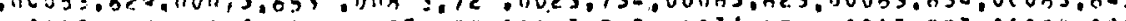
$.001+3.904 .01163 .9014 .0033 .020 .00153 .96 e$. 01033.976 .0013 .793 .06014 .005 $.0015 \% .024 .0(1974.075 .0010 .118 .00300 .106 .00034,185.00054 .343 .06294 .309$ .00074 .495 .00054 .651

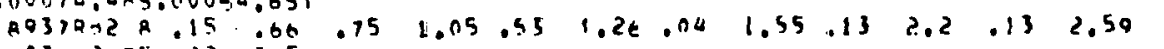

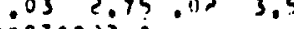

की

09348.12 .0067 .015 .09 .01

$0035=i 50$

$9015<42 ? 3.04 \quad .0 ! 3.15 \quad .11 \quad .65 \quad .12 \quad .09 \quad .23 \quad .07 \quad 036.147 .42$

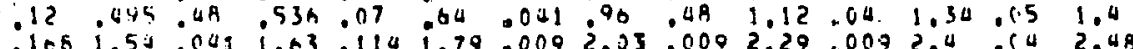

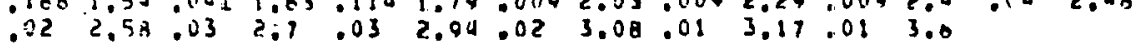

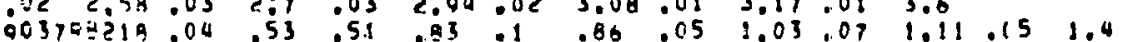

.031 .7 .021 2.2 .03 $2.51 .06 \quad 3.07 .1503 .30 .05 \quad 3.54 .114 .13$

.

003 in 4 is

$903913.09 \quad 015.97 \quad .002 .01 \quad .482$

ovjor $22,01-2, i n-1.04-31,734$

$9130 \times-2$ :

0

$91325225.15 \quad .045 .27 \quad .748 .03 \quad 093 \quad 01.025 .051 .413$

$013 u r 1$ ? 034.015 .05 is

$0130421.005 i .21$

abje<itz?

$0 \geq 3 ; i, j\}$

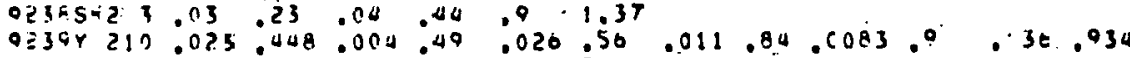
$.00261 .12 .0451 .4 .004 i .33 .30052,06$

$0330 \times 920$

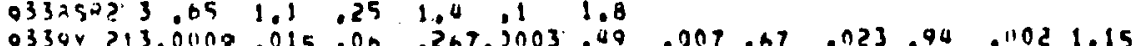

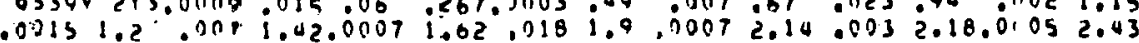
93402 दि?

9301.2411 .01 .017

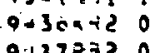

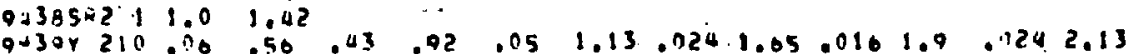
$015257.0072 .84 .013 \quad 3.06 .0113 .53$

0.1414013 . $11 \mathrm{r} .017 .0097 .407 .002 .871$

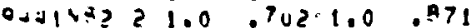

$9530 \times 250$

$05376+20$

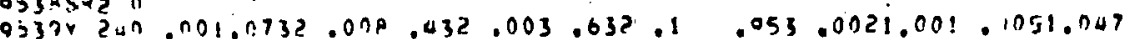

$3041.273 .0051 .274 .0201 .323,0041.356 .0041 .410 .0011 .445 .071 .019$

.03151 .654 .0011 .785 .0011 .798 .0021 .506 .0021 .814 .0031 .403 .0021 .005

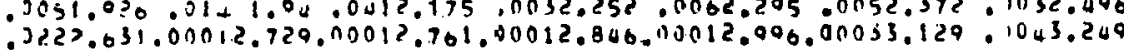

094

070

074

074

074

074

074

orsk

075

we 07sx

077x

$070 \mathrm{~N}$

080

HB OBIK

$0 B 1$

081

He OBटK

HR O OR3K

H2. $084 K$

$085 k$

OB7N

H) OBOK

H. 0Q0K

$091 x$
003
0.4

$093 \mathrm{~N}$
$094 \mathrm{~N}$

HI oajk

$076 k$

095

$092 \mathrm{~N}$
$009 \mathrm{~N}$

1000

- iolk

ini

OH. $103 \mathrm{U}$

i SSN

$100 \mathrm{~N}$

HB ligk
HA lonk

108

H2, 110K

A IIK

IIUN

$115 N$

$: 110 x$

110

110
116 
.0023 .05 .0223 .576 .00013 .684 .00013 .387 .00013 .922 .00014 .068

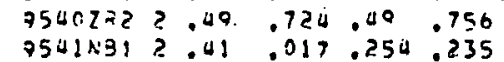

$054 ! v a ? 1.00 \div .765$

$9030 y \geq 31.0 \quad .7 \quad .4 \quad 1.0 \quad .33 \quad 1.5$

904108210.044 .215 .029 .241 .329 .349 .035 .372 .202 .450 .037 .48 .570 .500 .055 .72 .975 .778 .145 .811 .209 .851 .008 .94 .00051 .03 $.5151 .00 .19 n 1.2 \quad .0251 .5$

Q $93, \times 2 ? 0$

$0737+\pi$ in

$97335=50$

973945

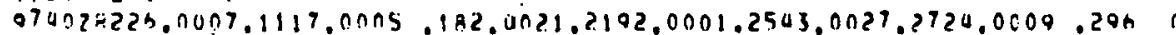
.0007 .331 .021 .3555 .0027 .401 .057 .5078 .009 .513 .015 .6024 .0622 .0903

.011 .7030 .09 .9432 .0075 .2048 .0028 .8290 .0037 .8548 .0029 .0711 .00051 .021 .0271 .100 .0111 .148 .0111 .276 .0151 .303 .0151 .75 .00451 .852

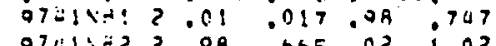

$97610.32 .08 \quad .665 .02 \quad 1.02$

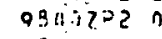

onding

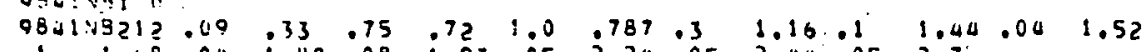

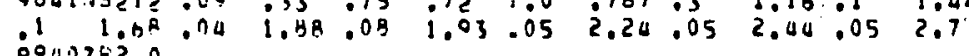

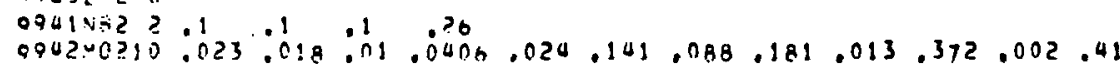

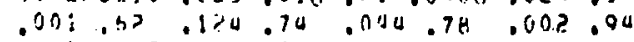

दQjicis? 0.075 .018 .0 .14

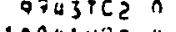

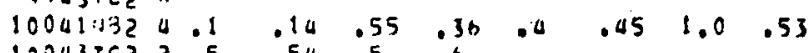

$\begin{array}{lllll}1004302 & .5 & .54 & .55 & .56\end{array}$

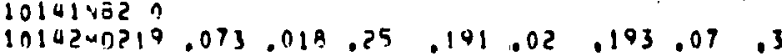

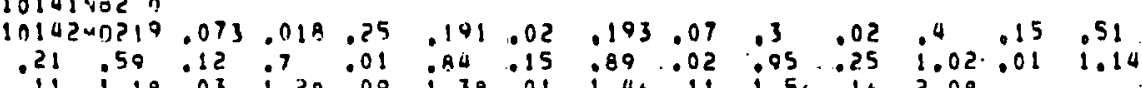

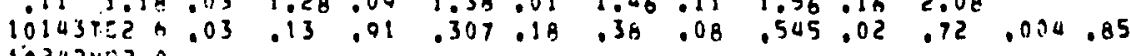

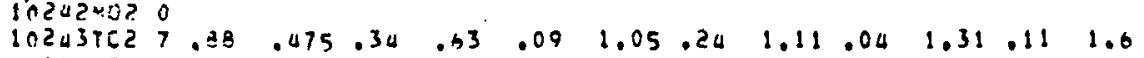
$116,2.10$

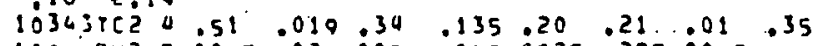

$103408427.0047 .02 .0036: 053.0027 .297 .0035 .045 .88 .498 .0077 .557$ 00647.61

$103452412.12 \quad .02 \quad .000 .04$

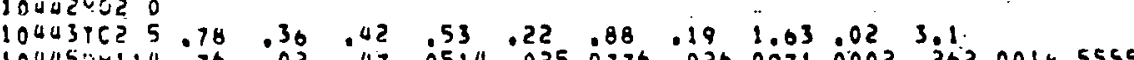

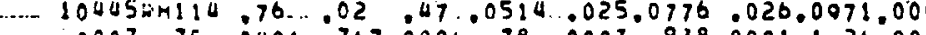
.0007 .75 .0001 .737 .0000 .78 .0003 .938 .03018 .26 .00011 .34 .00361 .53 i. $00011,950+2$

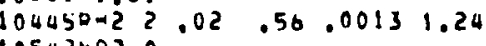

105437021000

$\begin{array}{llllllllllll}.02 & 0553 & .03 & .0752 .08 & .0823 & .3 & .1070 .02 & .1135 & .03 & .127\end{array}$

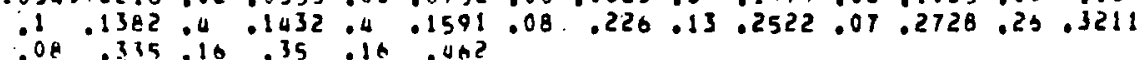

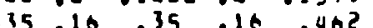

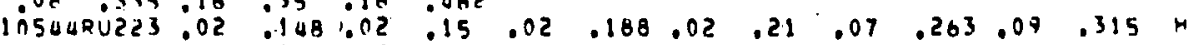

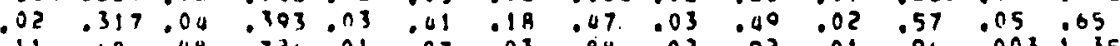

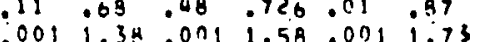

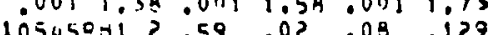

105452420.0034 .021 .0003 .0382 .0017 .2805 .054 .3063 .191 .3193 .0034 .4427

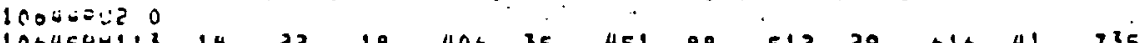
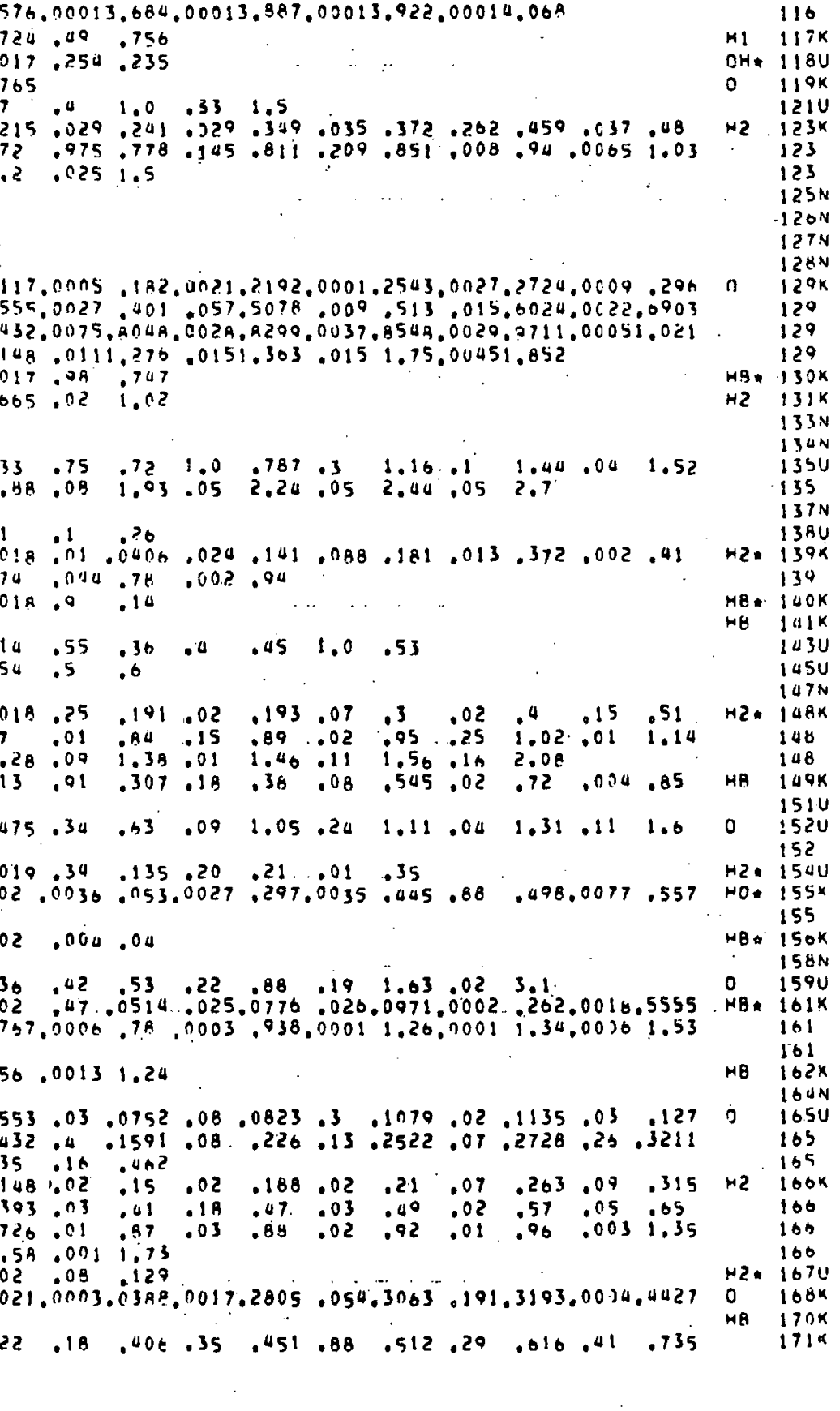
.02052 .105 .00072 .148 .00042 .156 .00352 .212 .00942 .507 .00082 .552 .06032 .577 .00242 .686 .00052 .723 .00002 .752 .00052 .705 .00012 .776 .00012 .804 .00392 .829 .00012 .830 .10022 .853 .00012 .853 .00022 .884 .00012 .921 .00022 .96 .00013 .375 113409020

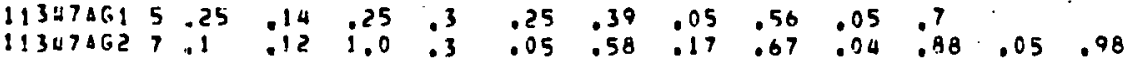

$.001,18$

$113480 \mathrm{CD} i$ ?

$\begin{array}{lllll}11 & 02083 & .001 & .265\end{array}$

$11407462,1.0,57$

$1140.1914 .01 \quad 024.17 \quad 102.033 .558 .035 .720$

$11+40105190017,020$

1151100020

$11507 A G 10$

$\begin{array}{lllllllllllll}11547.46222 & .01 & .11 & .12 & .14 & .02 & .17 & .49 & .22 & .07 & .24 & .13 & .28\end{array}$

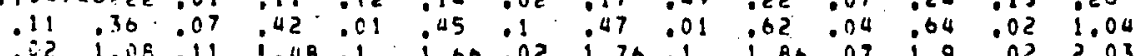

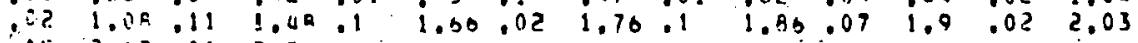
$152.12 .012,5$

Iisuecei 0.0003 .162.0038 .495.0039 .035.00031.13.00871.20.00.12 1.42 115450620.0006 .035 .005 .23 .0065 .20 .02 .262 .1 .40 .235 .53 $115441: 112.33 .024 .5 \quad .335$

115401950

$1160740 ? ? 1.0 .52 \quad .21,7$

201

201

$\mathrm{HB}$

$203 k$

5040

205

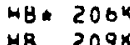

40
40.5

HB. $212 K$

HI $213 K$

21 $4 \mathrm{~N}$

H2 $216 \mathrm{~K}$

216

210

296

H2. $211 \mathrm{~K}$

$H$ ? $218 \mathrm{~K}$

HB. $219 K$

HB 2205

5251

. YE TEXT ISOTOPE MAS A MALF-LIFE OF SU MINUTES.

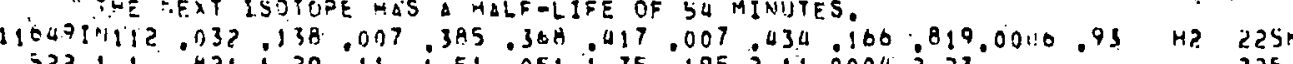

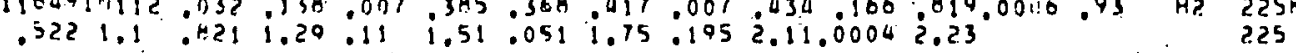

$\begin{array}{ll}0 & 240 K \\ \text { WH } & 250 K\end{array}$ 


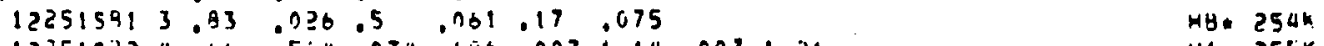

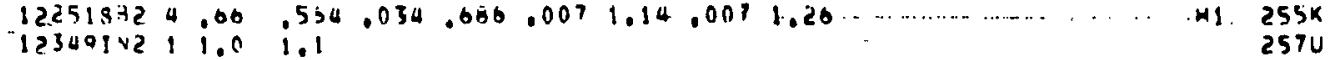

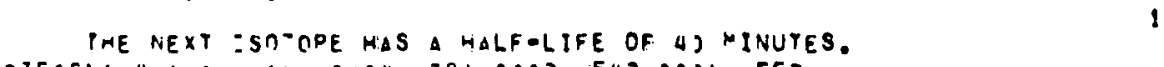

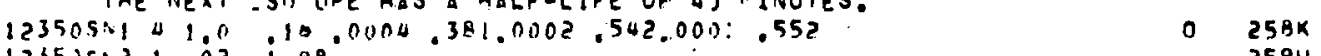

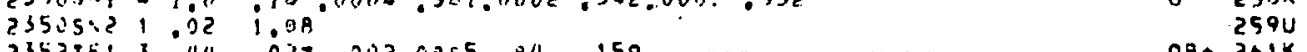

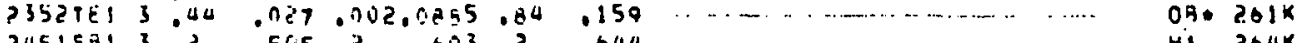

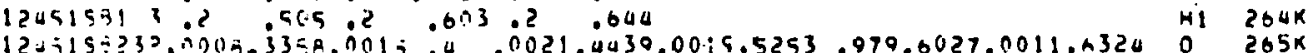

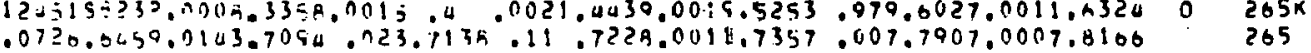

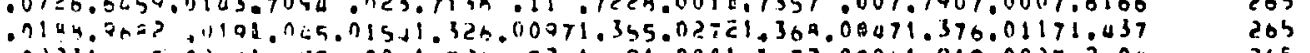

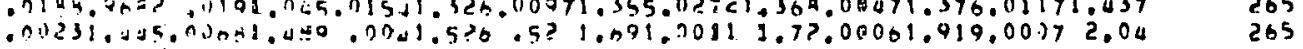

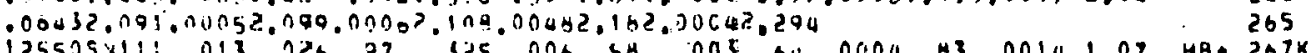

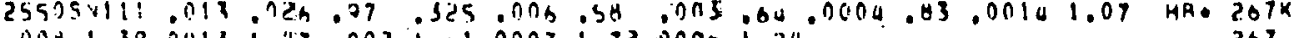

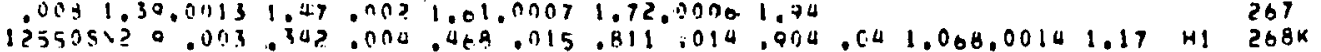

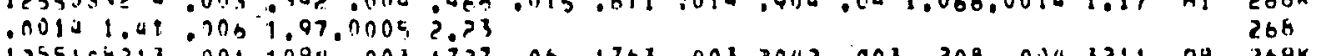

?55154313.001.1000.001.1727.06.1763.003.2042.003 .208.0.14.3211 03 289x

il?

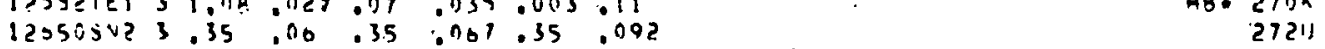

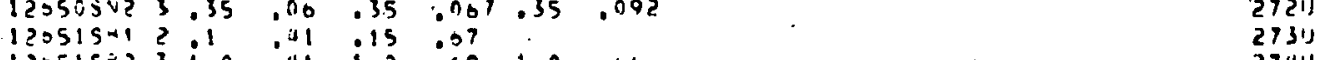

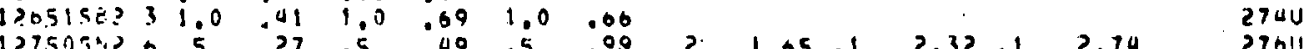

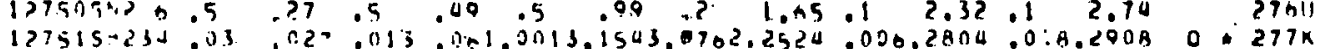

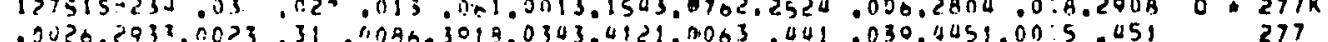

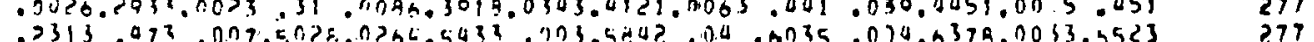

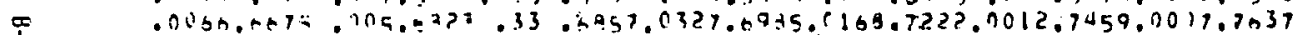

35.

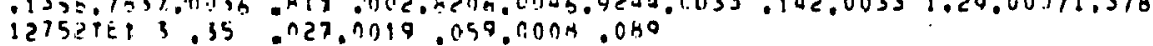

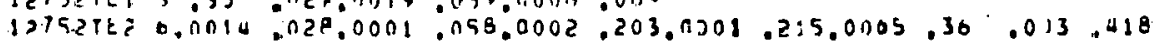

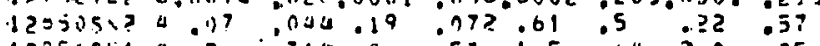

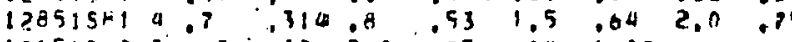

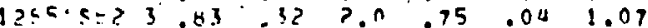

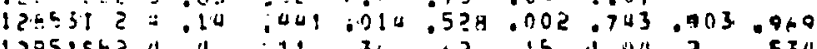

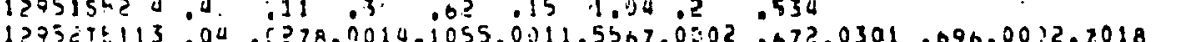

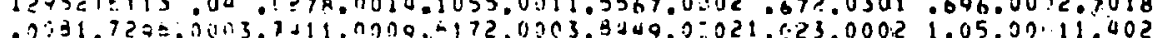

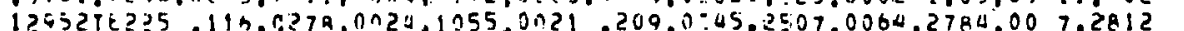

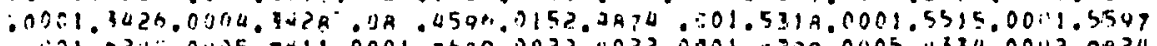
. An03i.084.0025i.12,00019.233.00011, 201.00011,204

$1705312 ? .7 \quad .029 .09 .00$

$13.4525 \div 0 \%$

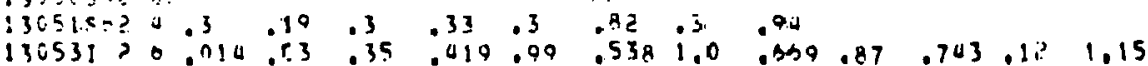

$131515 \div 23.37$.4 .48 05

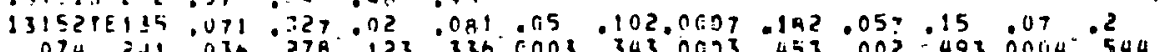

007 .

.

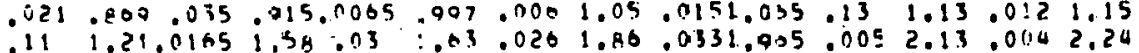

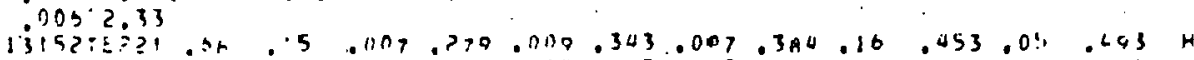

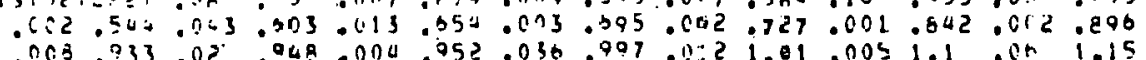
. 191.3

H1 2A3K

5870

2870
$298 K$

588
288

$289 x$

289

H1. $290 \mathrm{~K}$

?92N

HA. $295 x$

Hi $297 x$

598
$298 \pi$ 

$.0021 .7 ? .0131 .91 .0131 .99 .0032 .08 .0022 .16 .00052 .55 .00022 .08$ 133515820

1535215103.00 .79 .9741 .0020 .0855 .0108 .084 .0216 .0009 .0058 .1043 .0432 .1089 $.0054 .1711 .0133 \mathrm{~h} .732 .0114 .1245 .0043 .1032 .0022 .1982 .0151 .2134 .0014 .2208$

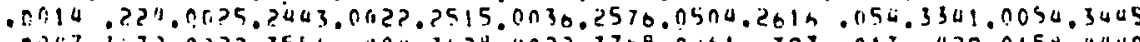
$.007 .3472 .0023 .3550 .004 .36>4.0022 .3708 .0141 .397 .013 .020 .0150 .0440$

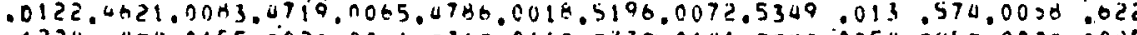

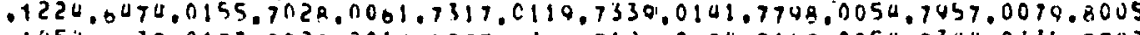
.115 .2539.0173. abra. .0097. cáco .00341 .537 .00791 .547 .02411 .643 .0041 .704 .00471 .840 .01942 .005 .0052 .028 ioret?

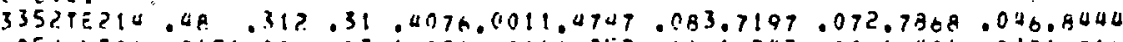
.050 .0309 .0451 .001 .0341 .021 .0141 .252 .111 .333 .0021 .406 .0321 .718 i 353,05 ?

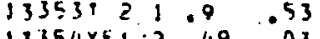

$13354 \times 81$ ? .49 . 13 is

.031.01724.01700.37.081.0000.161

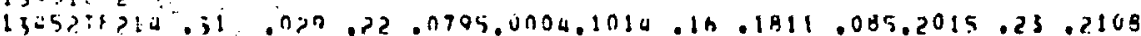

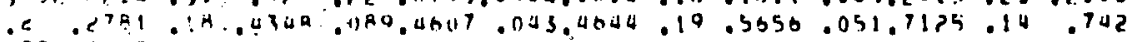

$13453 f^{2} 25.052 .135 \mathrm{~h} .005 .1824 .018 .2343 .083 .0053 .05 .4327 .071 .5413$

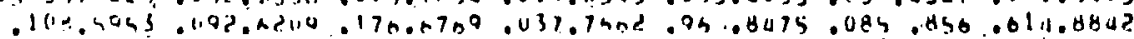
.015 .011 .010 .921 .020 .909 .003 .979 .0171 .04 .1311 .073 .0001 .130 $0241.459 .0361 .0 ? 1.0231,93,0531.81 j .0252 .41$ ?

134550313 ring

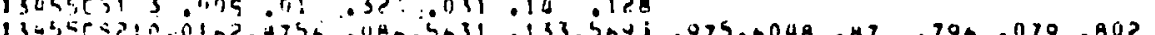

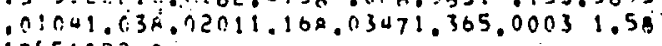

135515? 0 ?

13दsitt? 0 .

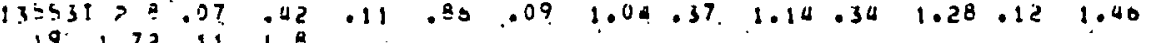

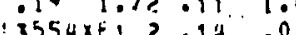

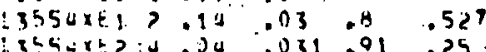

35456520

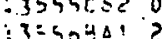

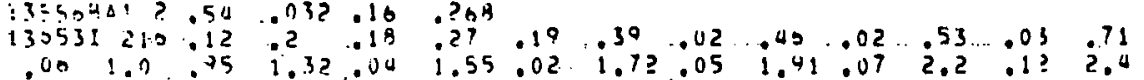

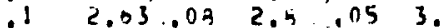

lis5ses;io.130.032.11 .057.06 .045.073.153.164.176.13 .273 .53 .34

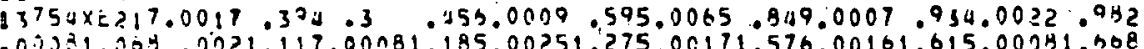
- D.j.

13755e 300

$131564: 12$.04 .05? .87 .652

$13+531 \geq 0$

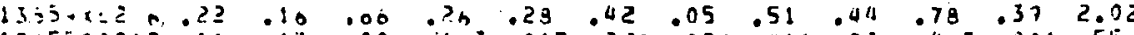

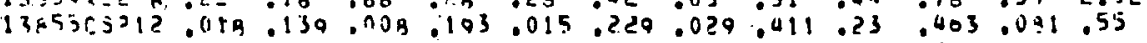

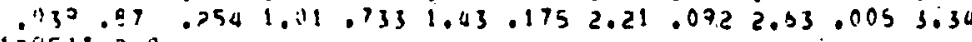


$13954 \times 2219.035 .031 .0064 .121 .249 .1749 .71 \quad .210 .02 \quad .2255 .099 .2899$

.234 .2907 .0033 .339 .1049 .353 .078 .3942 .014 .456 .014 .491 .021 .584

060.500 .244 .013 .000 .723 .012 .732 .032 .783

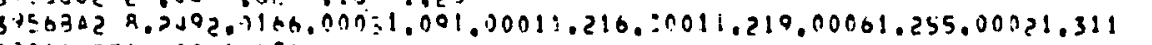

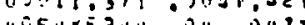

$01030.0794 .02 .0977 .08 \quad 103.13 .1116 .15 .1175$

01 .

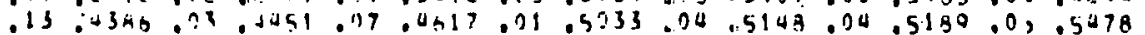

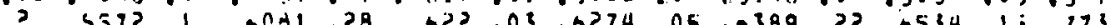

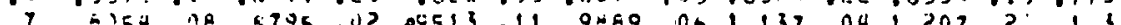

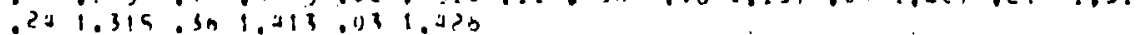

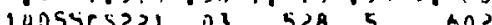

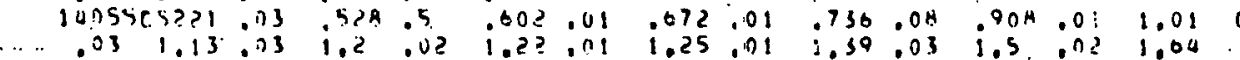

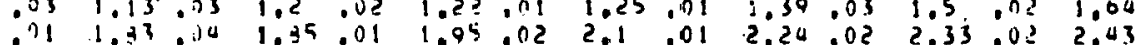

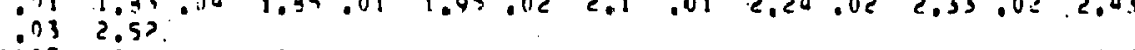




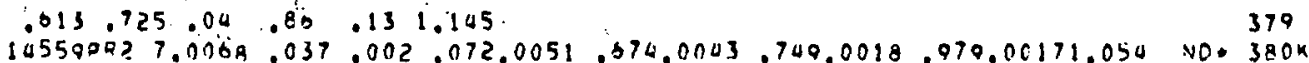

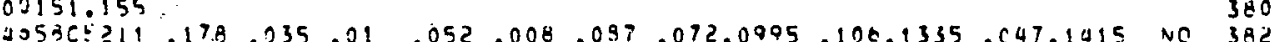

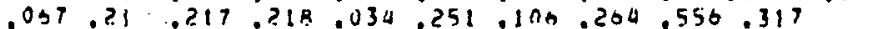
$1.45570424 ? .024 .037 .238 .4536 .005 .535 .0084 .501 .013 .50 .058 .002$ ND $383 \times$ .08 .711 .0070 .736 .130 .7362 .009 .17 .133 .70 .049 .024 .031 .017 .0271 .061 .0101 .144 .0131 .233 .0211 .284 .0401 .333 .11 .378 .0481 .452 .0251 .47 .01841 .408 .321 .526 .0171 .00 .0141 .645 .021 .745 .01541 .01

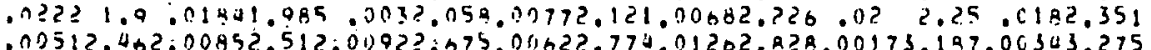
$.03512 .402 .00852 .512 .01992 ? .575 .01162 ? .774 .01202,828.00173 .197 .06343 .275$ 00173.365

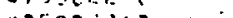

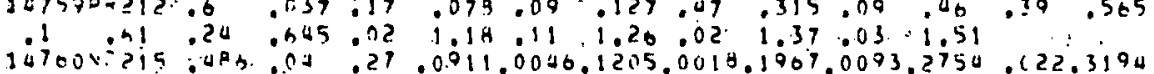

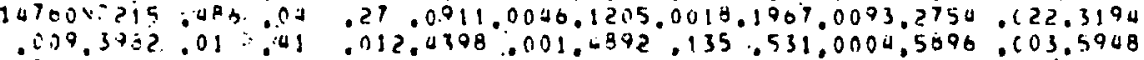
$.7003 .5704: 009,4859$

i० 70100 , 0

1470254 ?

14358650

$104500725.65^{\circ}, 005.003 .049$

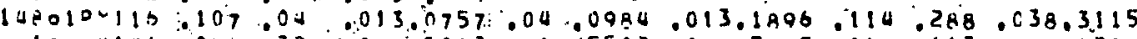
$.13 .4141 .000 .4324 .0771 .5013 .91,5502.08 .5005 .00 .0113 .60 .0209$ $.32 .725, .2 .015 ? 2.21 .010$

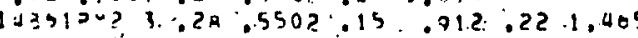

$1490040210.012 .06 .022 .076 .019 .042 .18=.114 .04 \quad .156 .022 .188$ .003 .109 .27 .01 .00011 .227 .04 .024 .26 .0200 .05 .0327 .011 .340

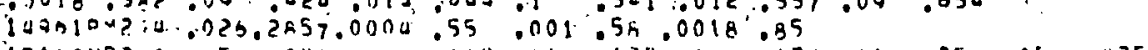

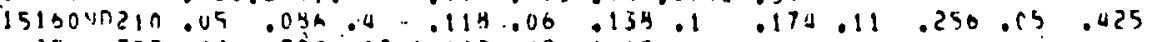

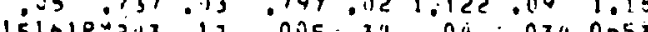

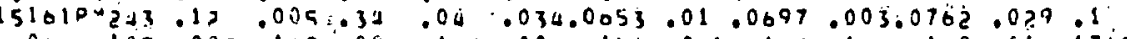

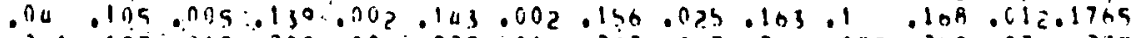
.

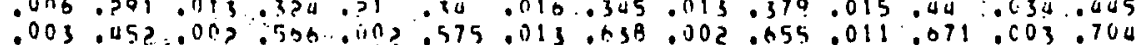

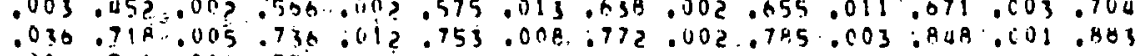

0.00 .0006 .023

$15261042 ? .5 .122 .5 \quad .245$

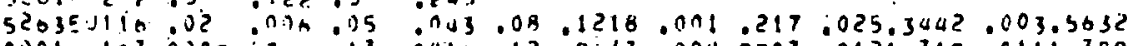

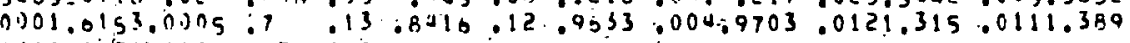
i 53035030.000151 .56 .00011 .03

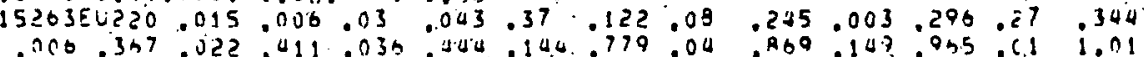

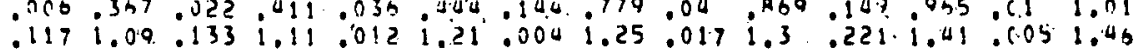

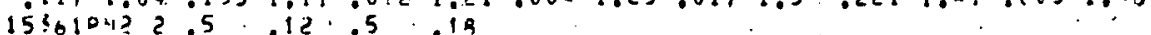

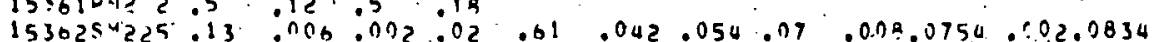
202.0905 008.0974.28 .103.0001.152.0004.165.003.173.0003 4t2

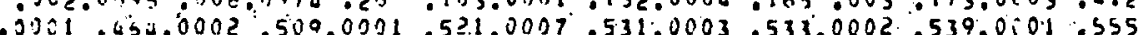

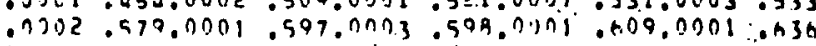

is 150105 ?

150435.0310038 .

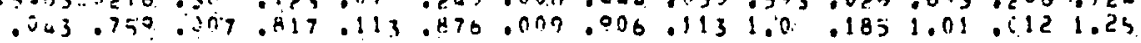

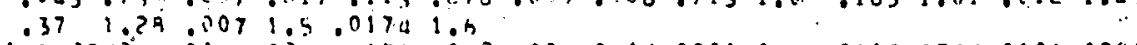
दa 250 ?

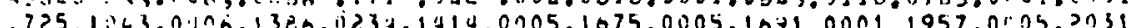

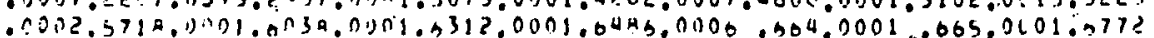


$411960211.0 \quad 1.20$

42:96 $7 . ? .002 .31 \quad .121 .5 ? 4$

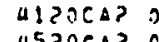

47?res: $3.05 \quad .49 .05 \cdot .815 \cdot .74 \cdot 1.31$

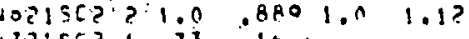

HB $462 \mathrm{~K}$

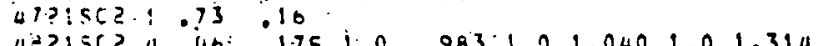

H.S $963 k$

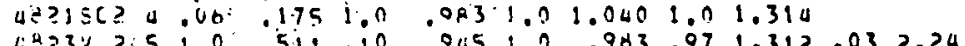

Wh $464 x$

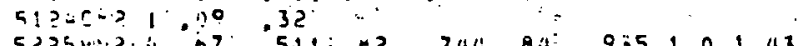

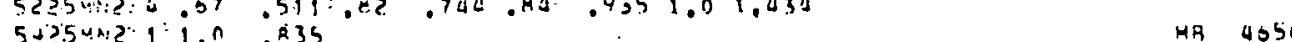

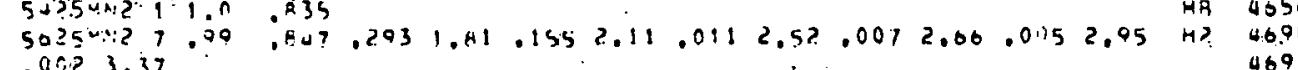

$\dot{0}=0,23,37$

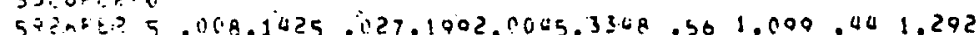

HH $467 \mathrm{~K}$

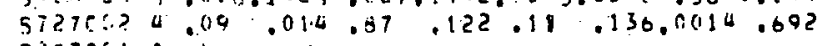

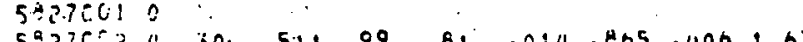

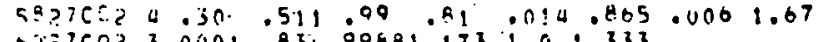

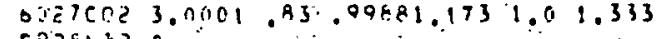

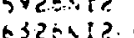

$0520.37 .045 .308 .101 .115-.251 .401$

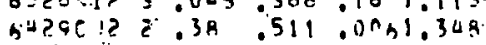

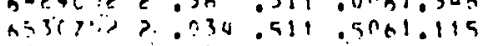

$0=3 \div 2 \cdots 1,05 ; 0,30$

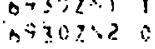

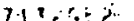

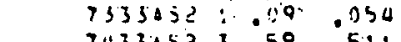

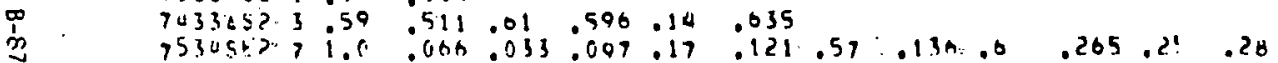

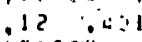

$8525=1.5 .10 \quad .15 \cdot .85 \quad .231$

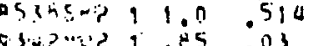

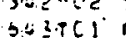

obuis? 5.05 .32.1.0 .779.84 .81 $1.0 .851 .16 \quad 1.12$

07.3961 .0

0.7431050

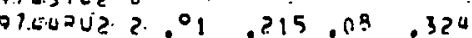

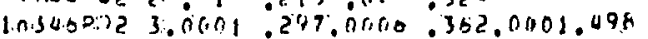

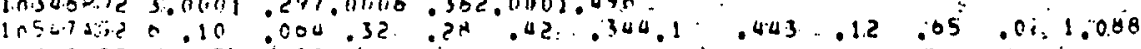

10.4ECO: 1.05 .0.8.

$11309901: 1.60 .393$

-. 11.35osis 1 .018 . 25.5

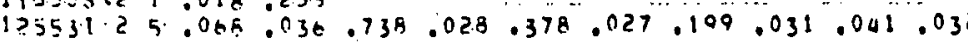

$120531222.34 .0396 .33 \quad .667$

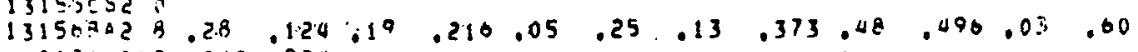

$.0131 .019 .000 .9 ? 0$

$19304032 ? .024 \dot{007} .55 \quad .099$

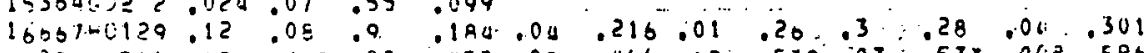

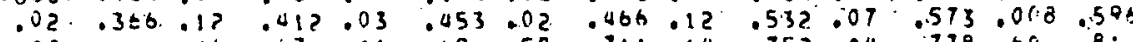

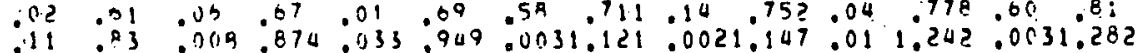

unmi.unz .0nal.uza

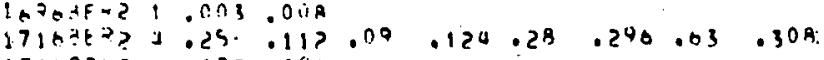

$\operatorname{lin} 0.10 ? 1.023 .0 \% 6$

111001020

$\because 0 \quad 472 K$

N1 $\begin{array}{ll}473 K \\ \text { iI) } 474 K\end{array}$

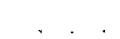


17570Y5? $3.010 .110 .031 .283 .00 \quad .396$

171716 ? ? $.028 .113 .061 \quad .208$

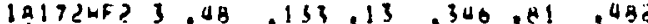

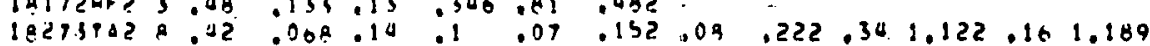

$.271 .532 .13 \div 1.231$

19194233.01 .006 .001 .136 .001 .152

192,945

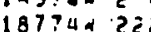

.009 .0002 .0362 .01 .050 .125 .072 .0005 .1060 .0009 .1137 No. $098 \mathrm{x}$

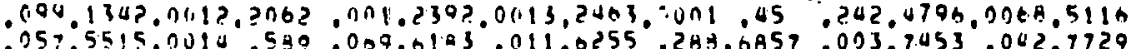
.0034 .6024 .002 .3796

1 1RTSRE 20

$17075023 \quad .00 \quad .139 .0003 .032 .0004,709$

18775 EEz 010

1A87SDE? 5.10 .155 .010 .478 .000 .633 .003 .829 .004 .032

la5T6.95? 2.40 . 646.14 .975

$10110.05: 11.0,070$

1017 nns $1.05,120$

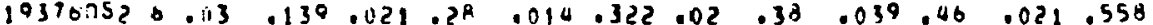

$100771520.51 \quad 157.37 \quad 37 \quad 30 \quad 43030.518 .72 \quad .56 .41604$

$192771227.29 .206 .30 \quad 308.81 .317 .49 .408 .04 .589 .09 .004$

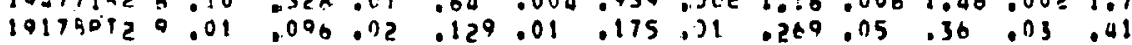

$.01 \quad .457 .09 \quad .430 .01 .024$

103720912
103780,5

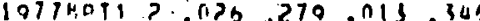

19775015 2 . 20 .011 . 10.101

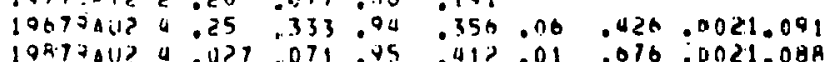

$10070 \Delta 425.31 .150 .08$ ? 502

107ACING1:2 4? $134.07 \% 270$

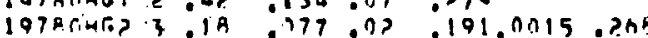

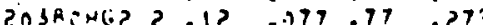

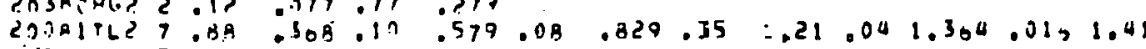

478

478

04051,0

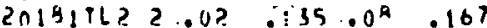

$20 \geq 011633.050 .030 .001 .522 .0007 .001$

actitits n

2034?052 3 . A1 .279.05 .401.009.04

21002052 1. .04 . Ca

21 $25: 203 ? 20,47 \quad 030.032,30$

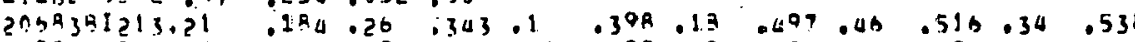

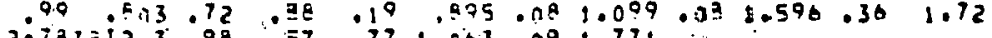

207335123.98 .57 .77 .1 .053 .09 .1 .771

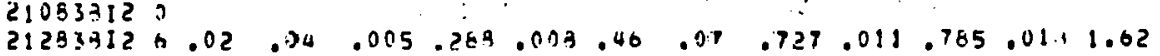

$510 B+802: 0$

5118545

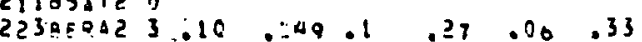

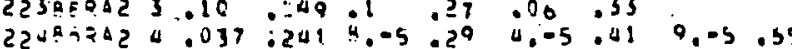

5250,745, $1,31.00$

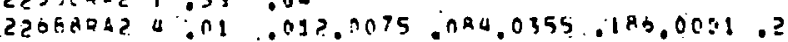

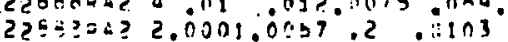

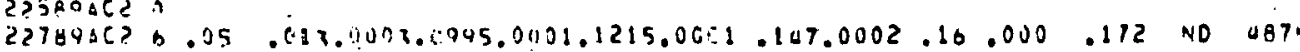

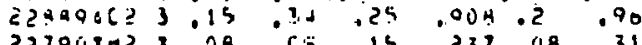

2270 istar $3.0 B$.C5 .15 .237.08 .31

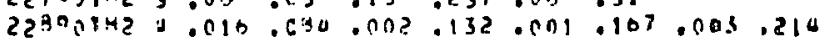

HB. $419 K$

MB. $480 \%$

$42 \quad 4 B 3 x$

MB 480K

Non पAS

481

$487 x$ 


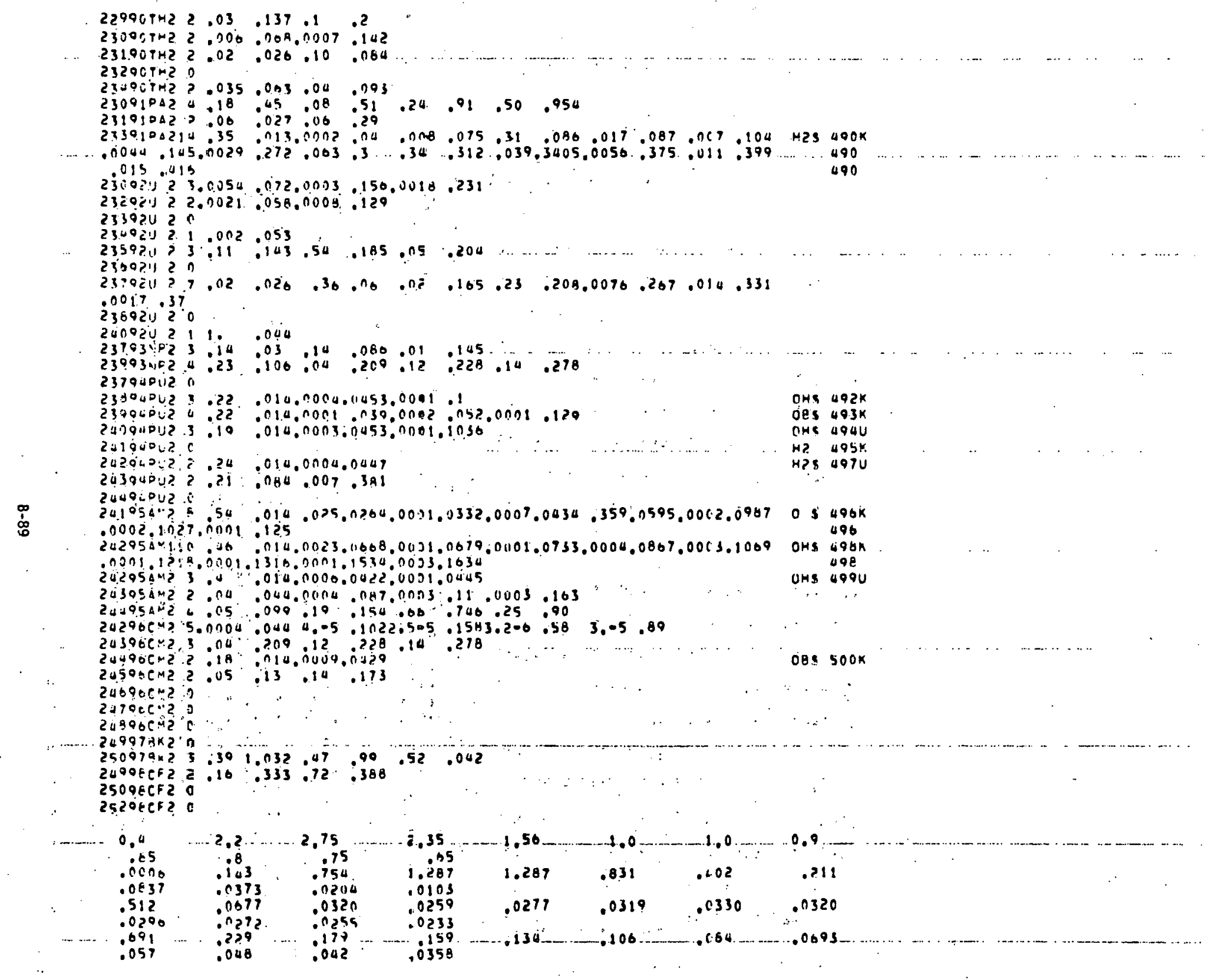




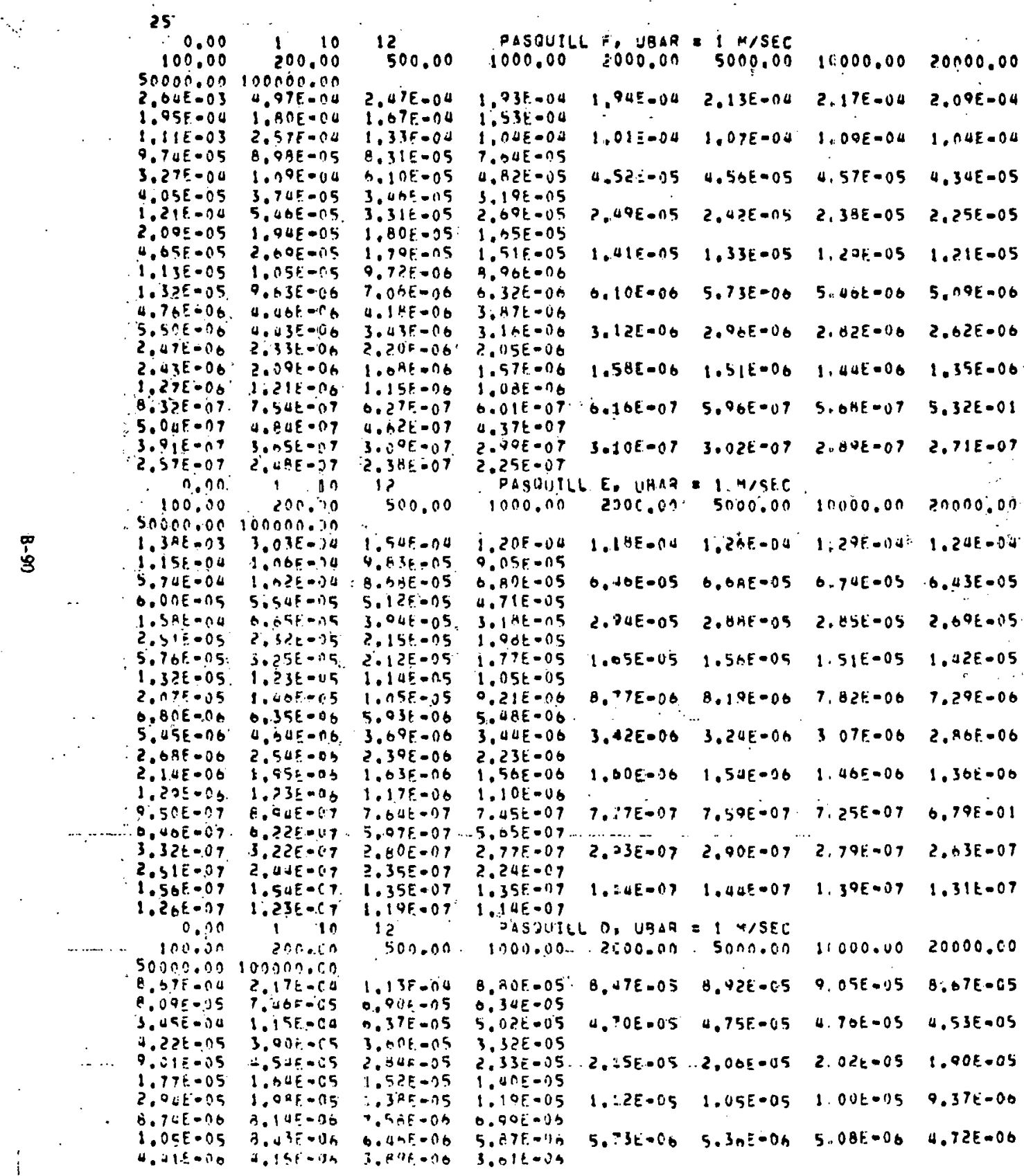


FIGURE B-9. Library BIVLIB Listing

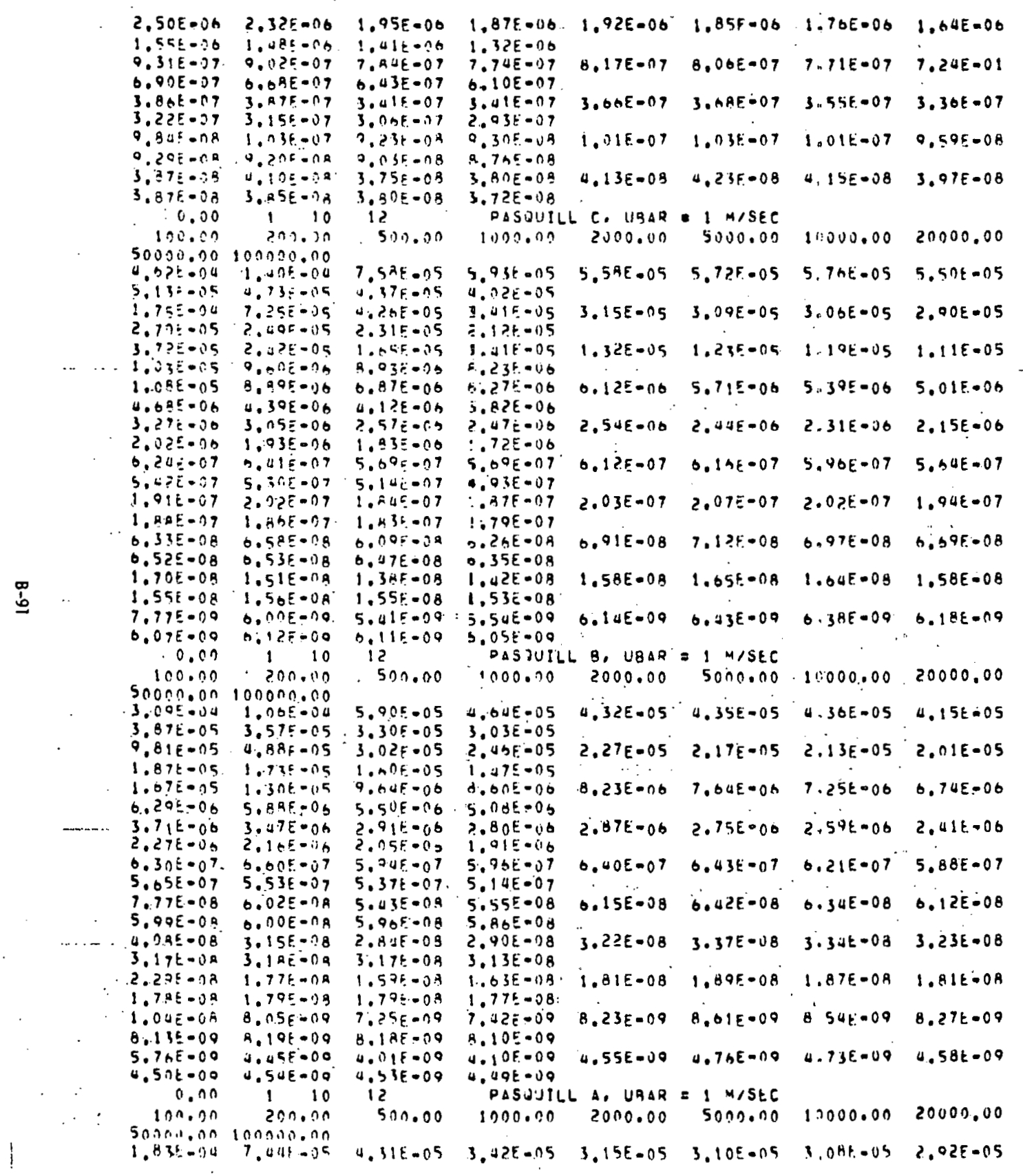




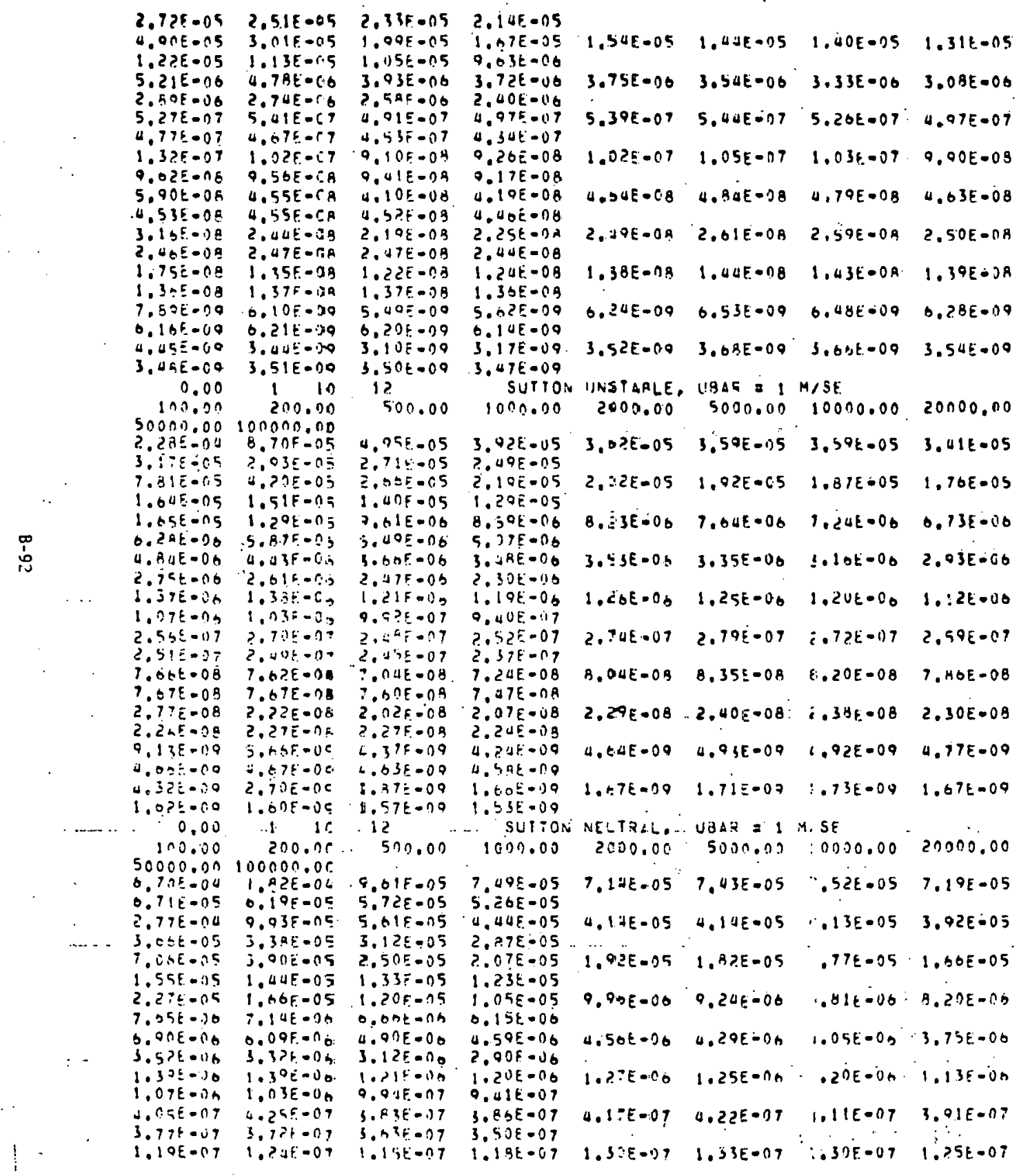




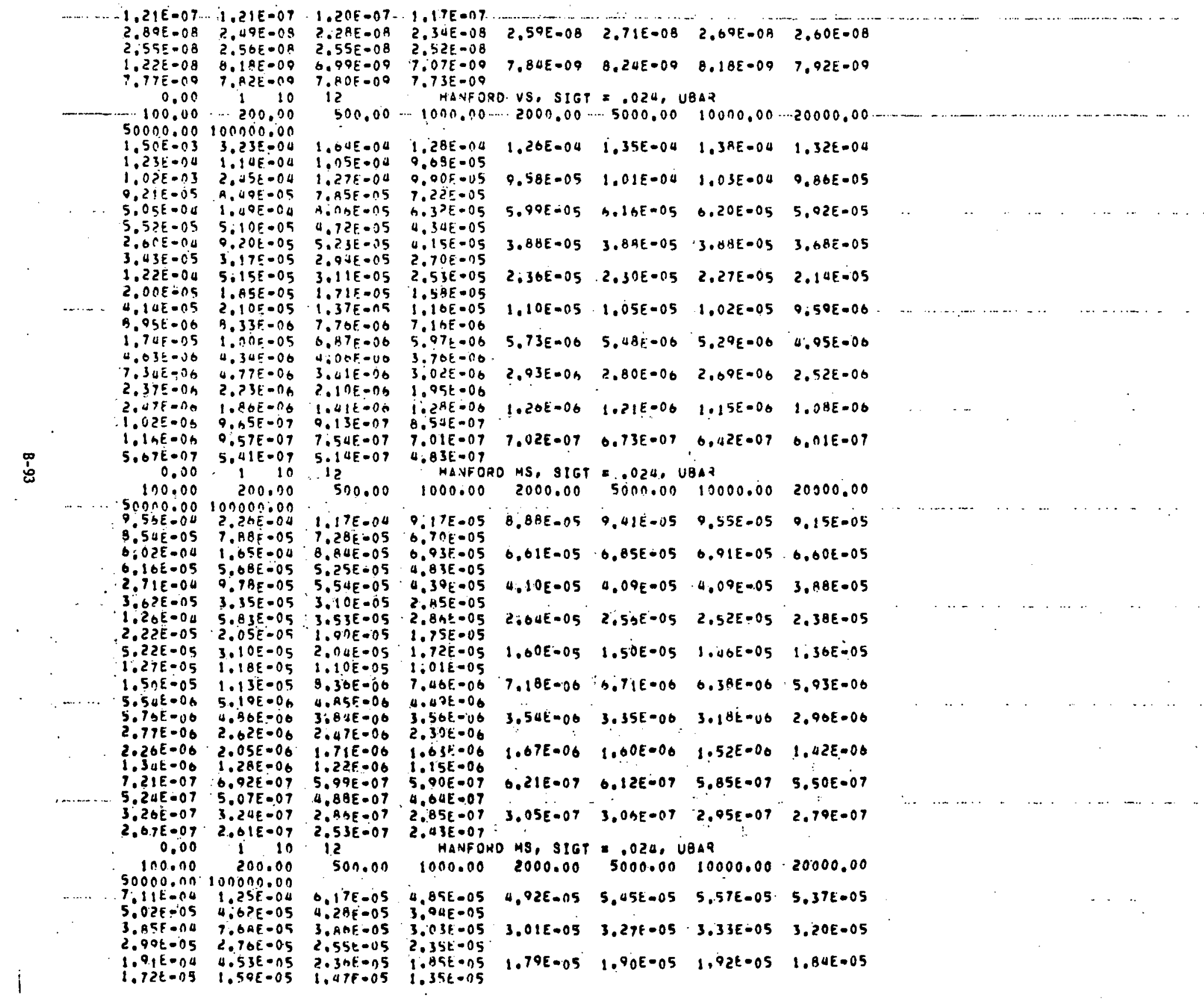




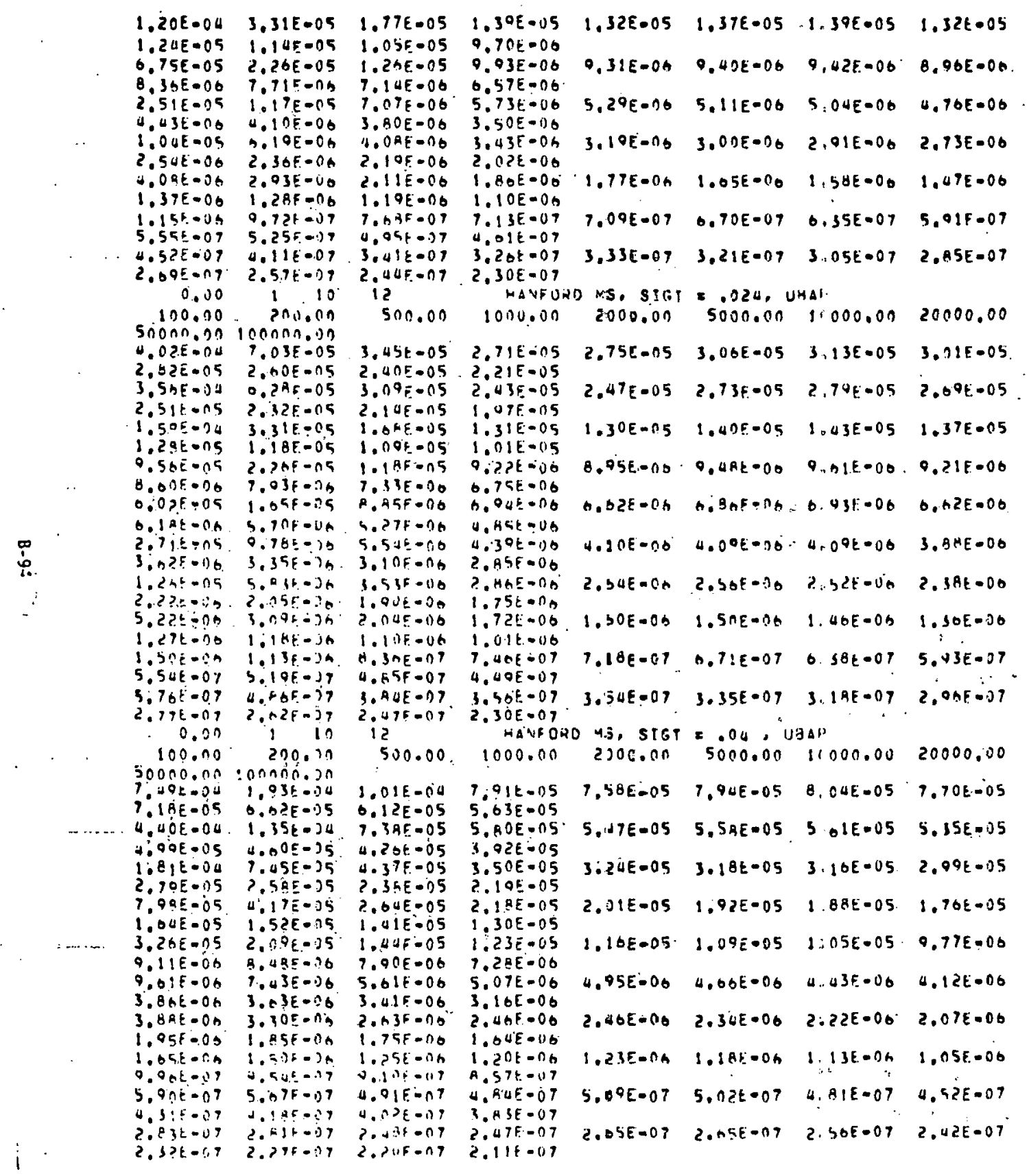




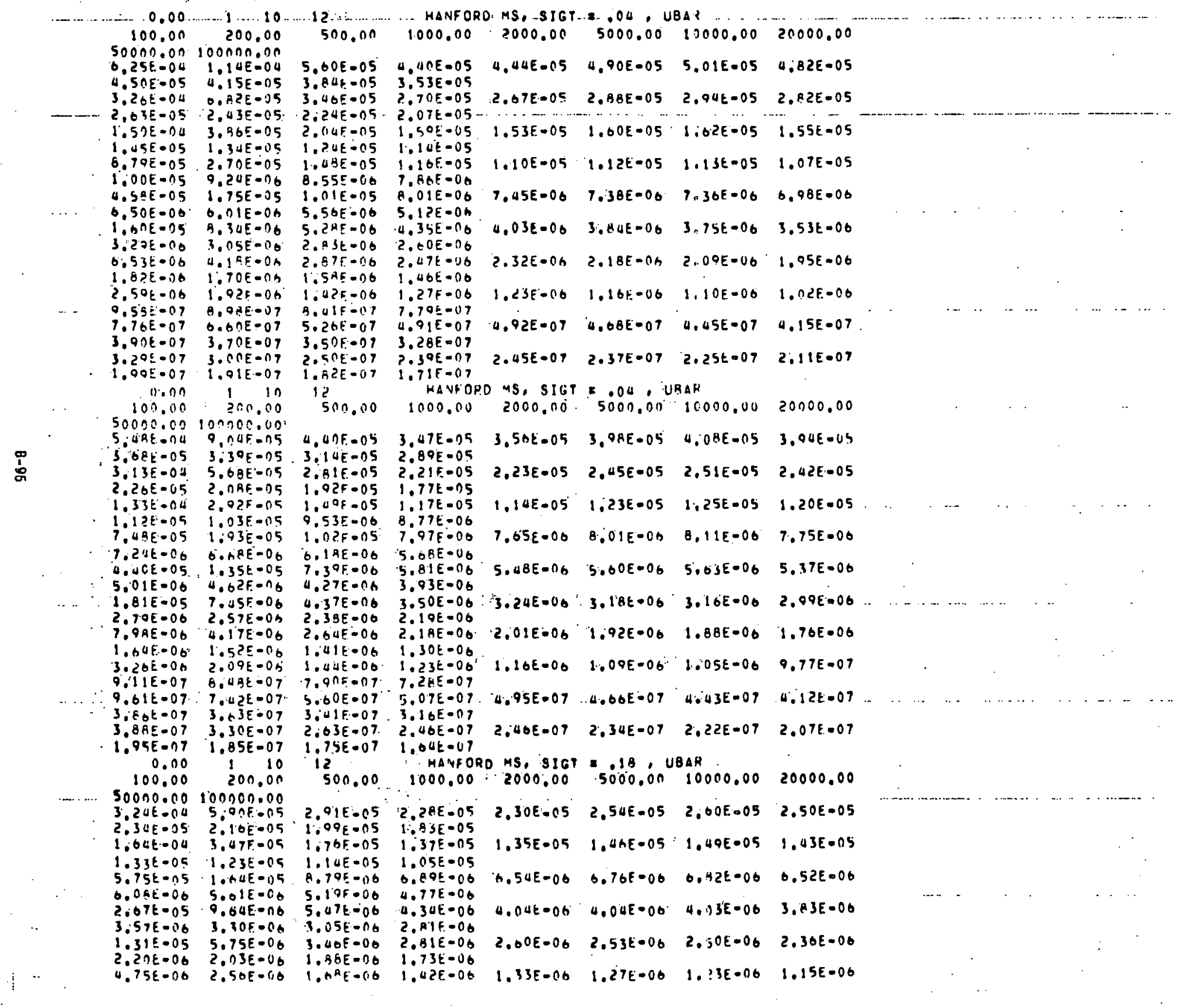




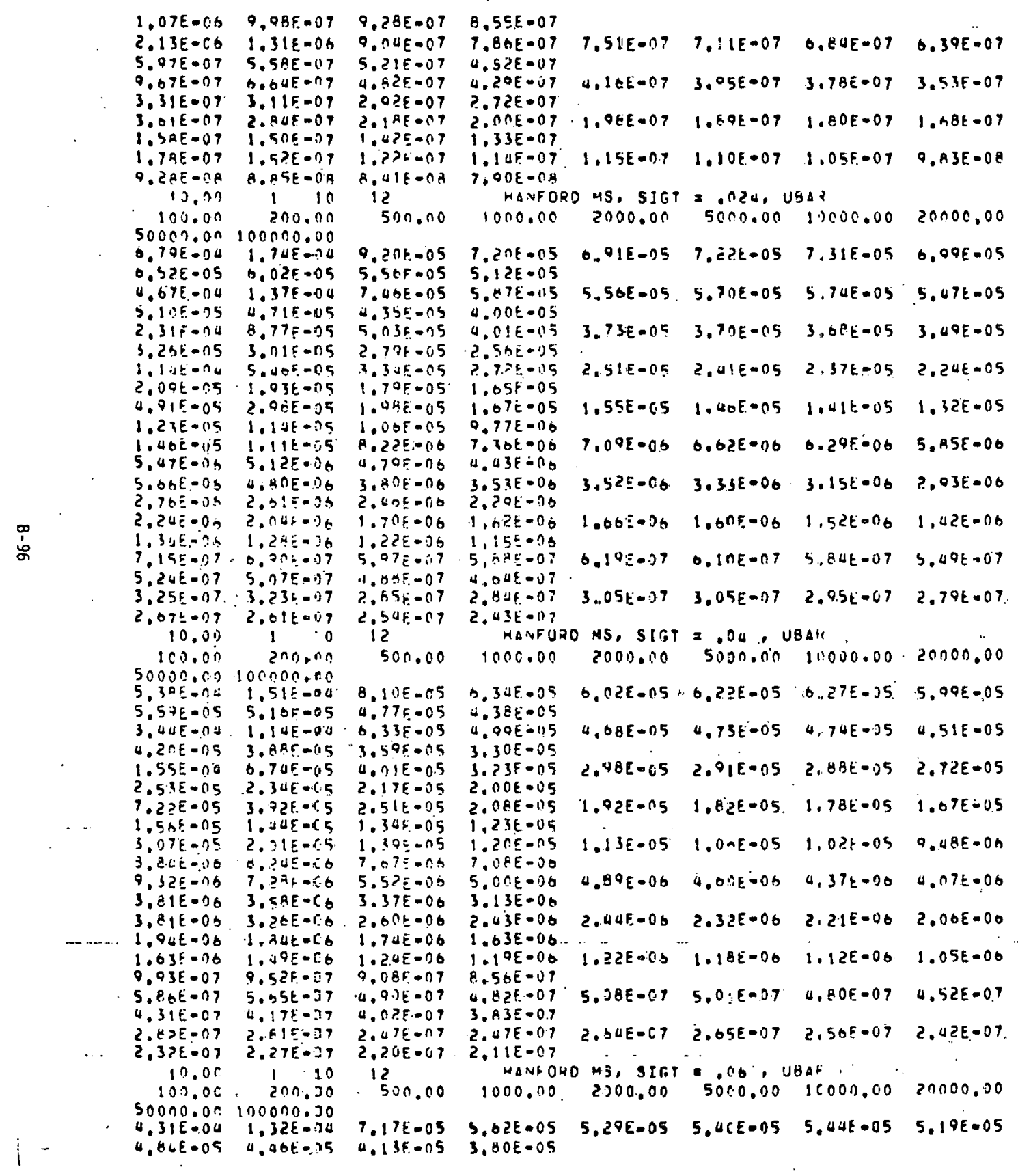




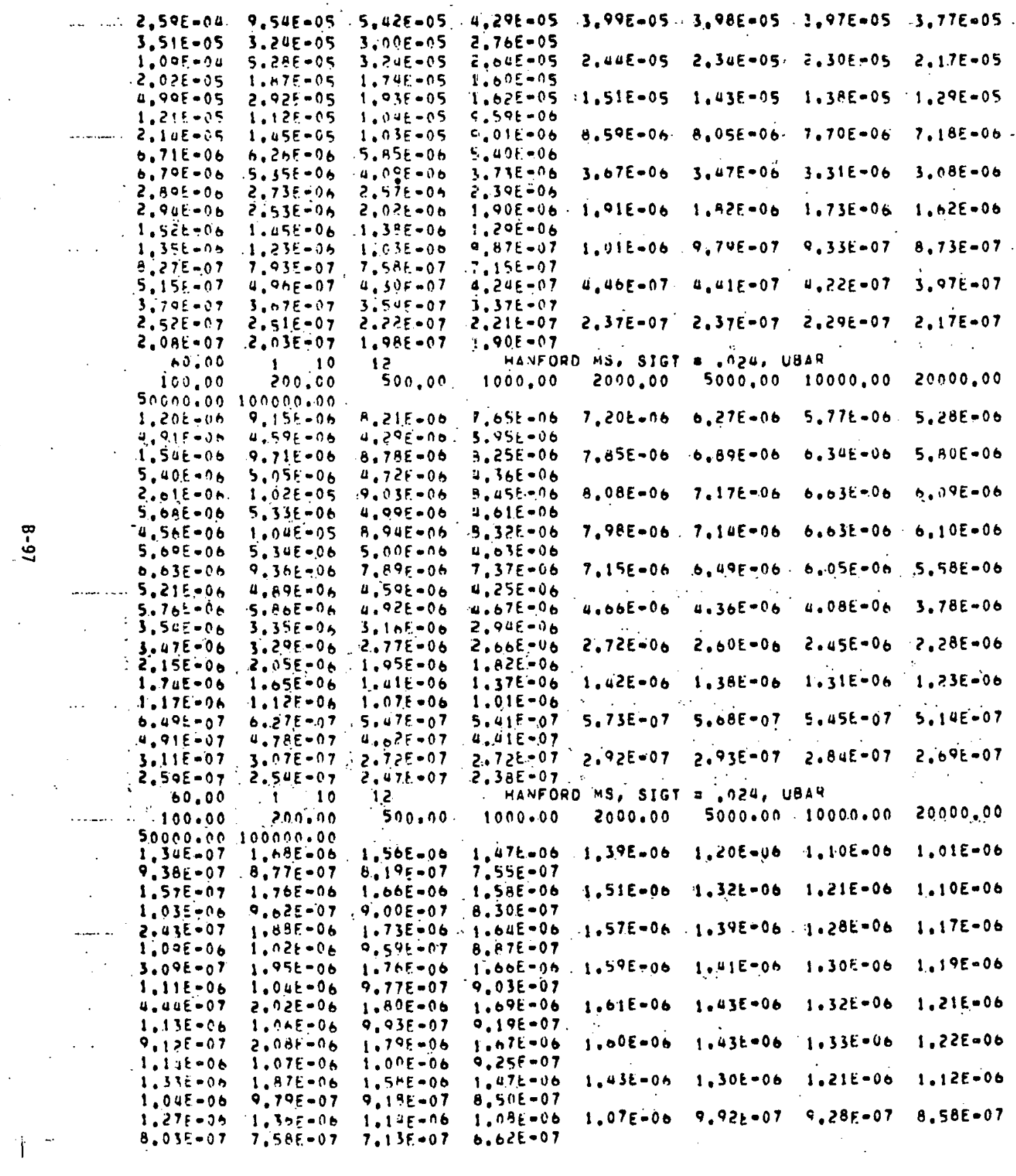




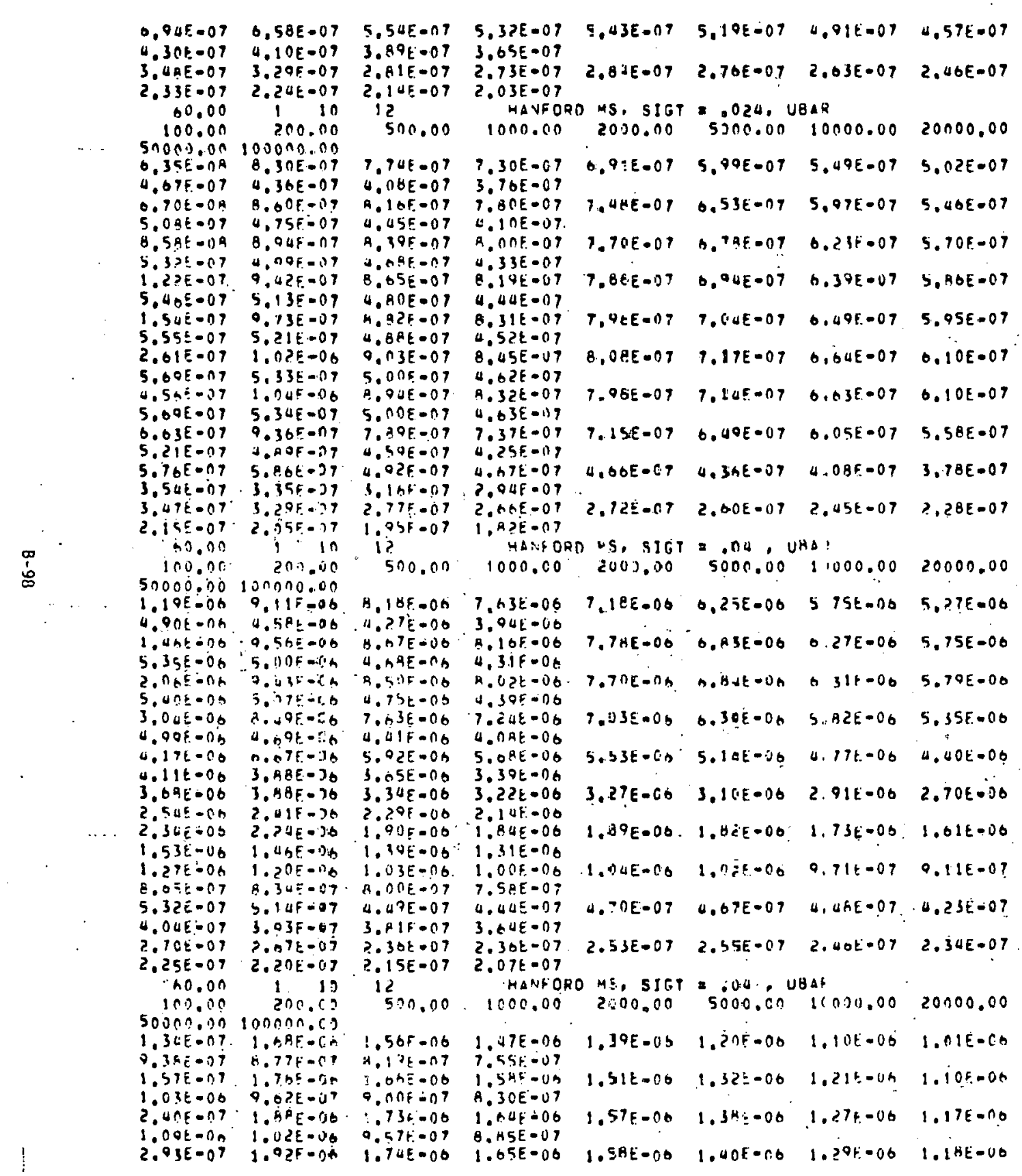




\begin{tabular}{|c|c|c|c|c|c|c|c|}
\hline $\begin{array}{l}-06 \\
-01\end{array}$ & $\begin{array}{l}1 . \cap D E-\cap B \\
1.9 \square E-\cap A\end{array}$ & $\begin{array}{l}9.68 F=177 \\
1.728=06\end{array}$ & $\begin{array}{l}07 \\
06\end{array}$ & $1.50 E-00$ & 8 & $\ln 29 E-06$ & $1.17 E-06$ \\
\hline & & .018 .07 & & & & & \\
\hline 8 & 1.75 & $\begin{array}{l}0 h \\
07\end{array}$ & $\begin{array}{l}6 \\
17\end{array}$ & n6 & $O E=0 B$ & on & $n 78$ \\
\hline & 1.3 ? & 10 & 1 & $13 E=06$ & $03 E-06$ & $0.55 E-07$ & $.79 E-07$ \\
\hline & 79 & & 7 & & & & \\
\hline & 0.15 & . & 7.5 & 07 & $E=07$ & 7 & $23 E-6$ \\
\hline & 5.55 & 7 & & & & & \\
\hline & 4.4 & 7 & $3.69 E$ & $3.79 E=07$ & 055.07 & $3.46 E \cdot 07$ & $.23 E=08$ \\
\hline & $\begin{array}{l}? .00 \\
2.0 \\
2.0\end{array}$ & $?$ & 7 & & & & \\
\hline & 1.e:E-ก) & - to & 1.5 & & & & \\
\hline & & & ORD & S. & & & \\
\hline & Ban.on & & sor & & 0 & & 0000.00 \\
\hline$?$ & $\sin a n \sin$ & & & & & & \\
\hline & f. & 7 & .3 & & & & \\
\hline & 4.35 & & & & & & \\
\hline & $\begin{array}{l}3,3 \\
4,7\end{array}$ & 4 & & & & & \\
\hline & $8.0: 5$. & 9.3 & .0 & 7.0 & 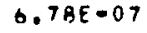 & & 5 \\
\hline & & & & & & & \\
\hline & $9.3^{8}$ & & & & & & \\
\hline & 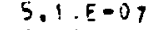 & & & & & & \\
\hline & 8. & & & & & & \\
\hline & 5.1 & & & & & & \\
\hline & 9.18 & 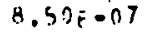 & & 7.71 & 9 & & O $A O F=07$ \\
\hline & $5 . n$ & & & & & & \\
\hline & S. & & & 7 & 07 & .07 & $5 E=$ \\
\hline & & & & & & & \\
\hline & $\because$ & & & 5.63 & 5.1 & 4. & 4. \\
\hline & 3.5 & $3 .+9$ & & & & & \\
\hline & & & & 3.27 & $3.10 E-07$ & 2.01 & $70 \mathrm{E}-$ \\
\hline & 20 & & & & -117 & & \\
\hline & $i .45$ & & & & & & \\
\hline & & & & & & & \\
\hline
\end{tabular}


APPENDIX C

INPUT PREPARATION 
APPENDIX C

\section{INPUT PREPARATION}

The preparation of input cards for SUBDOSA is described in this appendix. The input to Part II, BIVAR, is discussed first followed by input to PART III, SUBDOSA. No input cards are necessary for Part I, BELI, since data are supplied as data libraries generally stored on disk. The features and operation of BELI are described in Appendix A.

\section{PART II - BIVAR}

Program BIVAR calculates dose rate factors as a function of energy (described in text). The current version uses the energy groupings of photon probability library GISLIB (Appendix A).

Input to BIVAR is by card sets. Each set will command dose rate factor calculations for all combinations of up to six atmospheric stability categories, eight wind speeds, and ten downwind distances. The program varies distance, keeping wind speed and atmospheric stability constant. Wind speed is varied within each stability category. The dose rate factors calculated are automatically punched onto cards for use by SUBDOSA or for preparation of a dose rate factor library.

Six types of cards are used as input to BIVAR as described in Table C-1.

\section{PART III - SUBDOSA}

The program SUBDOSA uses NAMELIST for input of control variables and some data. The first card for each case is a title card followed by the NAMELIST cards. Additional cards depend on values submitted for control variables. A diagram of input logic is given in Figure $\mathrm{C}-\mathrm{l}$. The figure should be used with the description of card types and variables given in Table C-2 when preparing input to SUBDOSA. Dimensioned variables are indicated by their dimension in parentheses following the variable name. 
TABLE C-1. "BIVAR. Input Cards

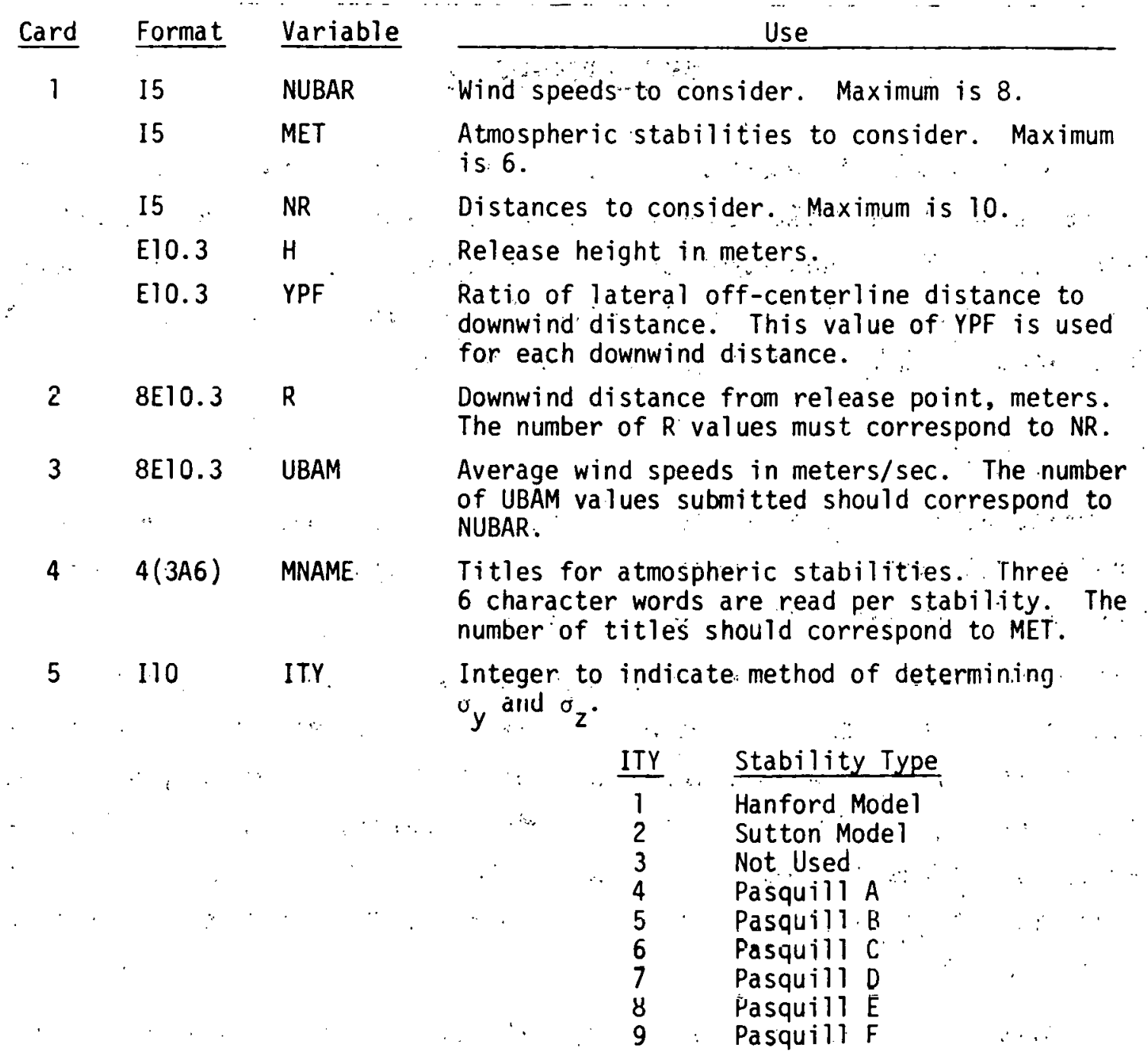

7E10.3 DUMMY Values read depend on value of ITY. For ITY = 1 (Hanford Model) inpul is:

$$
\begin{aligned}
& \text { DUMMY }(1)=a \\
& \text { DUMMY }(2)=b \\
& \text { DUMMY (3) - c } \\
& \text { DUMMY. (4) }=d \text {. } \\
& \text { DUMMY }(5)=k^{2} \\
& \text { (Also for ITY }=1 \text {, a card } 6 \text { is read.) } \\
& \text { For ITY }=2 \text { (Sutton Model): } \\
& \text { DUMMY (1) }=c_{y} \\
& \text { DUMMY (2) }=C_{Z}^{Y} \\
& \text { DUMMY }(3)=n \\
& \text { For ITY } \geq 3 \text {, DÜMY is not used. }
\end{aligned}
$$

$6 \quad 8 E 10.3 \quad S M$

Values of $\sigma_{\theta} \bar{u}$ are submitted for each wind specd. A card 6 is needed for each card 5 with ITY $=1$. 


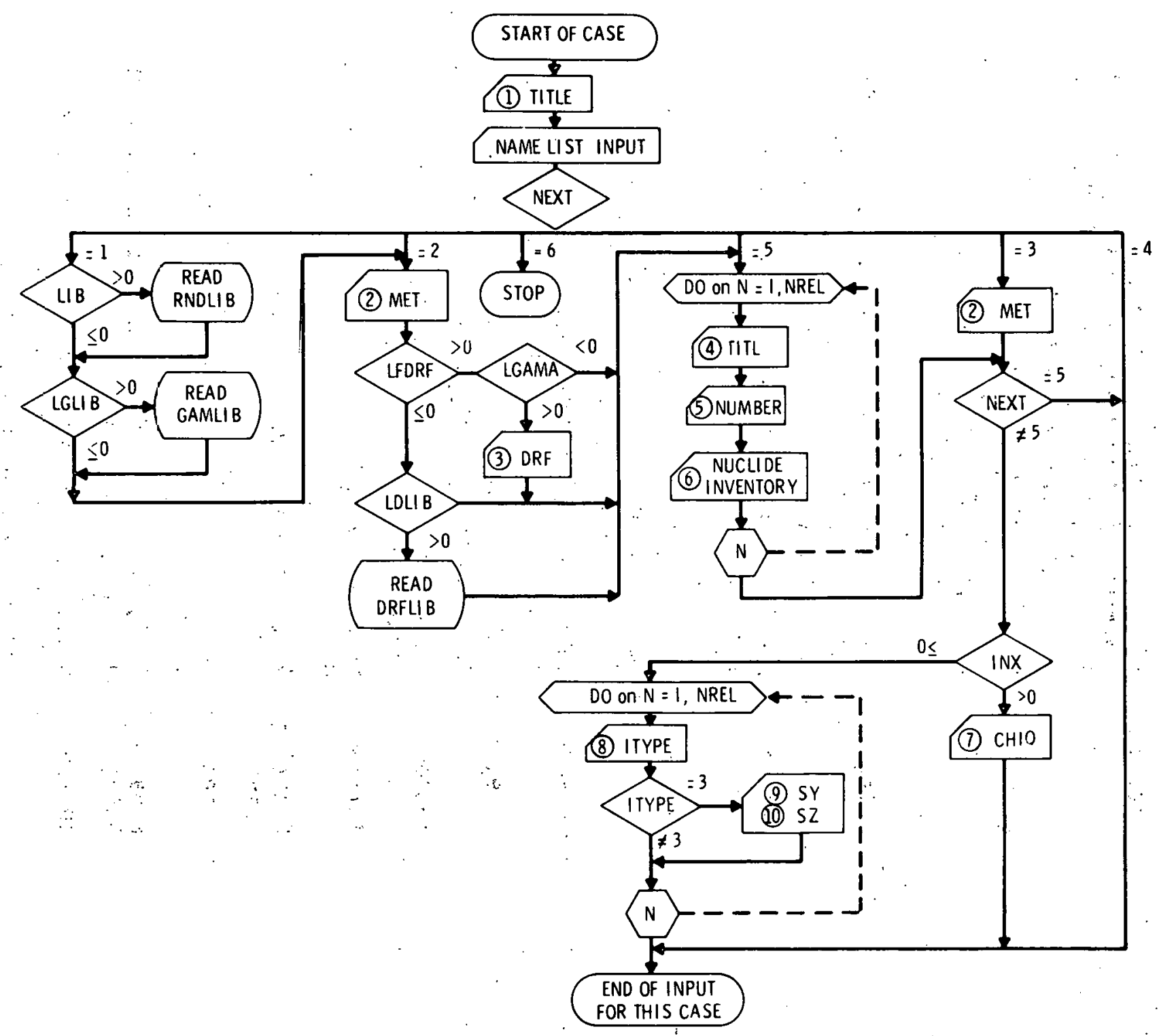

FIGURE C-1. Input Logic for Program SUBDOSA 
TABLE C-2. SUBDOSA Input Cards

\begin{tabular}{|c|c|c|c|}
\hline Card Type & Column & Format & Variable/Use \\
\hline 1 & $1-80$ & $8 A 10$ & $\begin{array}{l}\text { TITLE, title for current case to be printed } \\
\text { in-report heading. }\end{array}$ \\
\hline NAMELIST & & Ințeger. & $\begin{array}{l}\text { NEXT, input control integer. } \\
\text { 1. first case, initialize variables. } \\
2 \text { - input all but data libraries RNDLIB and } \\
\text { GAMLIB (DRFLIB may be read). } \\
3 \text { - read new meteorological conditions only } \\
\text { (DRF and/or XIQ). } \\
4 \text { - change NAMELIST variables only. } \\
5 \text { - read new nuclide inventories only. } \\
6 \text { - stop, end of run. }\end{array}$ \\
\hline NAMELIST & & Integer & $\begin{array}{l}\text { LIB, control integer }>0 \text {, read radionuclide } \\
\text { data library (NEXT }=1) ; \leq 0 \text {, do not read } \\
\text { the library. }\end{array}$ \\
\hline NAMELIST & & Integer & $\begin{array}{l}\text { LGLIB, control integer }>0 \text {, read gamma energy } \\
\text { library (NEXT }=1) ; \leq 0 \text {, do not read the } \\
\text { library. }\end{array}$ \\
\hline NAMELIST. & & $\begin{array}{l}\text { Integer } \\
\quad:\end{array}$ & $\begin{array}{l}\text { LDLIB, control integer }>0 \text {, read dose rate } \\
\text { factors from data library (NEXT }=1,2 \text {, or } \\
3 \text { ); } \leq 0 \text {; do not read the library. }\end{array}$ \\
\hline NAMEL IST & & Integer & $\begin{array}{l}\text { LBETA, control integer }>0 \text {, do beta dose } \\
\text { ralculation; }=0 \text {, no beta dose calculalion. }\end{array}$ \\
\hline NAMEL IST & & Integer & $\begin{array}{l}\text { LGAMA, control integer }>0 \text {, do gamma dose } \\
\text { calculation; }=0 \text {, no gamma dose calculation. }\end{array}$ \\
\hline NAMELIST & & Integer & $\begin{array}{l}\text { LCHIOQ, control integer, }>0, \text { calculate and } \\
\text { print } X / Q ; \leq 0 \text {, do not print } X / Q \text {. }\end{array}$ \\
\hline NAMEL IST & & Integer & $\begin{array}{l}\text { NREL, number of release periods, maximum is } \\
6 \text {. }\end{array}$ \\
\hline NAMELIST & & $\begin{array}{l}\text { Floating } \\
\text { Point }\end{array}$ & $\mathrm{RT}(6)$ release times in seconds. \\
\hline NAMELIST & & Integer & $\begin{array}{l}\text { NK, number of distances to consider, maximum } \\
\text { is } 10 \text {. }\end{array}$ \\
\hline NAMEL IST & & $\begin{array}{l}\text { Floating } \\
\text { Point }\end{array}$ & $\begin{array}{l}D(10) \text {, downwind distances (meters) at which } \\
\text { dose is to be calculated. }\end{array}$ \\
\hline NAMEL IST & & Integer & $\begin{array}{l}\text { NMET, number of weather stabilities to be } \\
\text { considered for each input period. NMET is } \\
\text { not currently in use and is set to } 1 .\end{array}$ \\
\hline
\end{tabular}


TABLE C-2. (Cont inued)

\begin{tabular}{|c|c|c|c|}
\hline Card Type & Column & prmat & riable/Use \\
\hline NAMEL IST & & $\begin{array}{l}\text { Floating } \\
\text { Point }\end{array}$ & UBAR, wind speed in meters $/ \mathrm{sec}$. \\
\hline NAMEL IST & & & $\begin{array}{l}H \text {, height of release point above ground } \\
\text { level, meters. }\end{array}$ \\
\hline NAMEL IST & & Integer & $\begin{array}{l}\text { ITZ, control integer to indicate when nuclide } \\
\text { decay is to be considered. ITZ }=0 \text {, for } \\
\text { inventories specified at start of accident } \\
\text { with decay considered to start of each } \\
\text { release period and during travel to exposure } \\
\text { point. ITZ }=1 \text {, for inventories specified } \\
\text { at start of each period with decay during } \\
\text { release and travel....ITZ }=2 \text {, for inven- } \\
\text { tories specified as time integral of release } \\
\text { during each release period with decay con- } \\
\text { sidered during travel only. }\end{array}$ \\
\hline NAMEL IST & & Integer & $\begin{array}{l}\text { NBD, number of tissue depths to consider } \\
\text { for beta dose calculation. The standard } \\
\text { beta energy } 1 \text { ibrary has data for tissue } \\
\text { depths of } 0,7,20 \text {, and } 100 \mathrm{mg} / \mathrm{cm}^{2} \text {. If } \\
\text { other tissue depths are to be considered, } \\
\text { a new library must be supplied with data } \\
\text { corresponding to the tissue depths desired } \\
\text { (Appendix A). Maximum for NBD is } 4 \text {. }\end{array}$ \\
\hline NAMEL IST & & Integer & $\begin{array}{l}\text { NGD, number of tissue depths to be considered } \\
\text { for the gamma dose calculation, maximum is } \\
3 \text {. The program uses default values of } 0 \text {, } \\
1 \text {; and } 5 \mathrm{~cm} \text { tissue depths. Other values } \\
\text { may be used by input of variable TDEP below. }\end{array}$ \\
\hline NAMEL IST & & $\begin{array}{l}\text { Floating } \\
\text { Point }\end{array}$ & $\begin{array}{l}\text { BDEP (4), beta dose tissue depth specifica- } \\
\text { tion to be used with NBDI below. BDEP is } \\
\text { used only for reports. Units of BDEP are } \\
\mathrm{mg} / \mathrm{cm}^{2} \text {. }\end{array}$ \\
\hline NAMELIST & & $\begin{array}{l}\text { Floating } \\
\text { Point }\end{array}$ & $\begin{array}{l}\text { TDEP (3), tissue depths for gamma dose cal- } \\
\text { culation, cm: Values. stored in data state- } \\
\text { ments are } 0,1 \text {, and } 5 \mathrm{~cm} \text {. }\end{array}$ \\
\hline NAMEL IST & & Integer & $\begin{array}{l}\text { NBDI. (4), index to indicate the relation } \\
\text { between the beta energy library data sets } \\
\text { (tissue depths) and the beta dose sets. For } \\
\text { example, to indicate a } 7 \mathrm{mg} / \mathrm{cm}^{2} \text { depth as } \\
\text { the first beta dose set } \mathrm{NBDI}(1)=2 \text { since } \\
7 \mathrm{mg} / \mathrm{cm}^{2} \text { is the second beta energy } 1 \text { ibrary } \\
\text { data set. The number of values submitted } \\
\text { must be at least equal to. NBD. }\end{array}$ \\
\hline
\end{tabular}




\section{TABLE C-2... (Continued)}

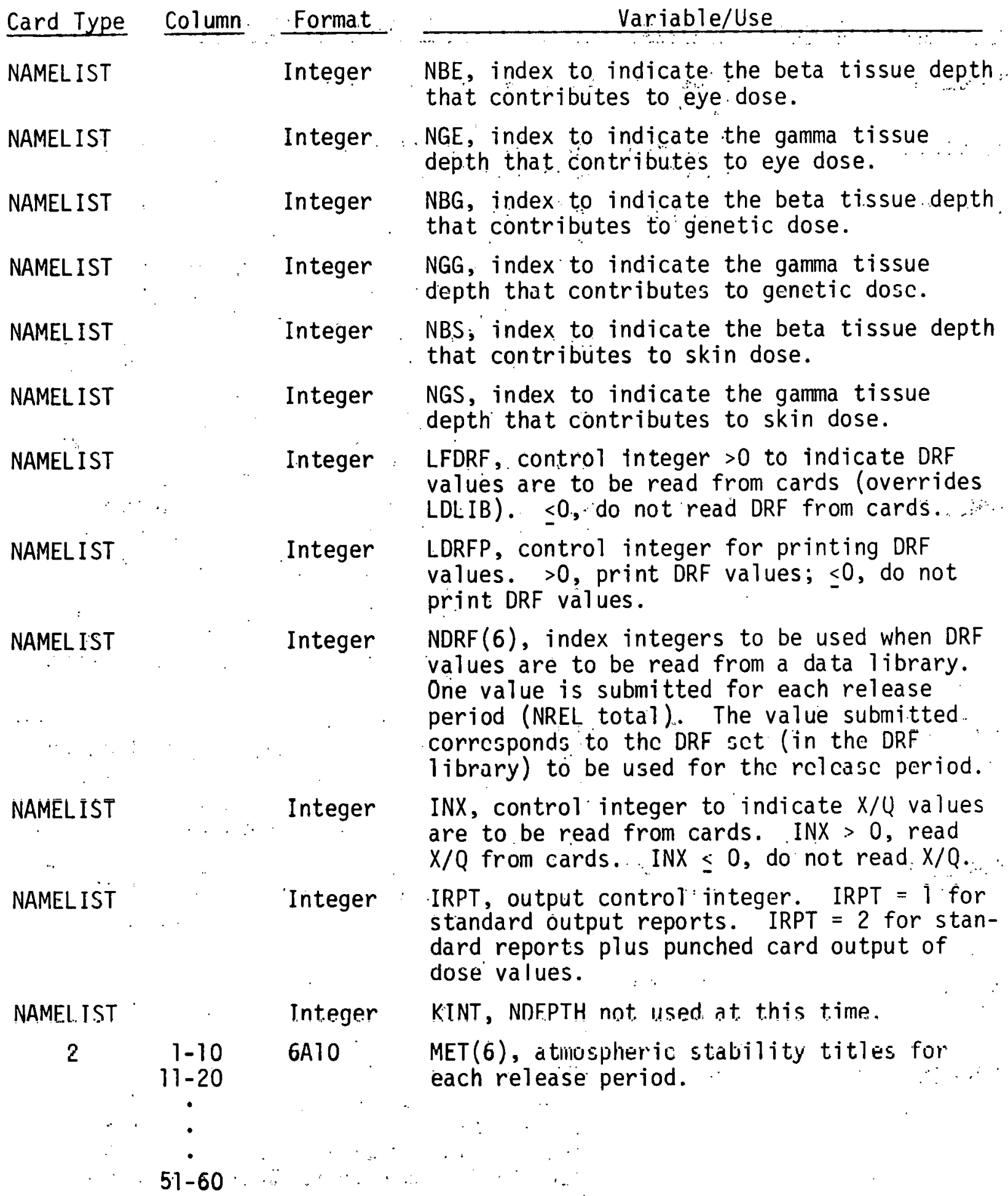


TABLE C-2. (Continued)

\begin{tabular}{|c|c|c|c|}
\hline Card Type & Column & Format & Variable/Use \\
\hline 3 & $\begin{array}{c}1-10 \\
11-20 \\
\vdots \\
71-80\end{array}$ & $8 \mathrm{E} 10.3^{\prime \prime}$ & $\begin{array}{l}\text { DRF }(1.20,1,6) \text { values submitted in sets for } \\
\text { each release period. Data within each } \\
\text { release period set are for distances cor- } \\
\text { responding to values supplied for D. Two } \\
\text { cards sare needed for each distance contain- } \\
\text { ing DRF values for each of the } 12 \text { energy } \\
\text { groups. }\end{array}$ \\
\hline 4 & $1-80$ & $8 A 10$ & $\begin{array}{l}\text { TITL, nuclide inventory title card for cur- } \\
\text { rent release period. }\end{array}$ \\
\hline 5 & $1-3$ & I3 & $\begin{array}{l}\text { NUMBER, number of nuclides to be read for } \\
\text { current inventory set. }\end{array}$ \\
\hline \multirow[t]{7}{*}{6} & $1-3$ & A3 & $\begin{array}{l}\text { NAME }(500) \text {, name of first nuclide, i.e., } \\
\mathrm{KR}^{*} \text { ( }{ }^{*} \text { indicates isomeric state). }\end{array}$ \\
\hline & $4-6$ & 13 & $M(500)$, a tomic weight of first nuclide. \\
\hline & $7-20$ & E14.0. & QUANTITY $(500)$ curies of first nuclide. \\
\hline & $21-23$ & A3 & NAME for second nuclide. \\
\hline & $24-26$ & I3 & M, a tomic weight for second nuclide. \\
\hline & $27-40$ & E14.0 & QUANTY, curies of second nuclide: \\
\hline & : & $\dot{0}$ & $\begin{array}{l}\text { Four nuclides are entered per card with } 20 \\
\text { columns for each as indicated above. }\end{array}$ \\
\hline 7 & $1-7$ & 10E7.2 & $\begin{array}{l}\text { CHIQ }(10,1,6) \text { values of XIQ, one card sub- } \\
\text { mitted for each release period. Each card } \\
\text { contains XIQ values corresponding to dis- } \\
\text { tances D. }\end{array}$ \\
\hline \multirow[t]{4}{*}{8} & & & $\begin{array}{l}\text { This card contains data used to calculate } \\
\text { XIQ values. }\end{array}$ \\
\hline & $1-5$ & I5 & $\begin{array}{l}\text { ITYPE, integer to indicate method of deter- } \\
\text { mining } \sigma_{y} \text { and } \sigma_{z} \text {. }\end{array}$ \\
\hline & & & Stability Type \\
\hline & , & $\cdots$ & $\begin{array}{ll}1 & \text { Sutton Equations } \\
2 & \text { Hanford Equations } \\
3 & \text { Input oy arld } \sigma \mathrm{Z} \\
4 & \text { Pasquill } \mathrm{A} \\
5 & \text { Pasquill } \mathrm{B} \\
6 & \text { Pasquill } \mathrm{C} \\
7 & \text { Pasquill } \mathrm{D} \\
8 & \text { : } \\
9 & \text { Pasquill } \mathrm{E} \\
9 & \text { Pasquill } \mathrm{F}\end{array}$ \\
\hline
\end{tabular}




\section{TABLE C-2: (Continued)}

Card Type Column Format.

8

6-11 I5
$11-20 \quad 7 F 10.2$

9

$1-10$
$11-20$
$\vdots$
$\vdots$
$71-80$
$1-10$
$11-20$

10
Variable/Use

IPR, control integer for printing values of $\because \sigma_{Y}$ and $\sigma_{Z} . \quad I P R>0$ for printing values; $I P R \leq 0$, do not print values.

DUMMY (7). "Values read depend on the value of ITYPE: "For ITYPE $=1$, Sutton equations, input is:

DUMMY (1) $=C_{y}$

DUMMY (2) $=C_{Z}$

DUMMY $(3)=n$

For ITYPE $=2$, Hanford equations; input is:

DUMMY $(1)=a$

DUMMY (2) $=b^{\text {: }}$

DUMMY $(3)=c$

DUMMY $(4)=d$

DUMMY (5) $=k^{2}$

DUMMY $(6)=\cdot \sigma_{\theta} \bar{u}$

DUMMY is not used for ITYPE $\geq 3$.

$\operatorname{SY}(10,1,6)$ values of $\sigma_{Y}$ to be read when ITYPE. $=3$ on card 8. Values are submitted corresponding to values of distance $D$.

Input under the NAMELIST routine consists of one or more cards with variables listed by their names as indicated above. The first NAMELIST card must be blank in column 1 with \$INPUT in columns $2-7$, followed by at least one blank, followed by data items. The data items are separated by a comma, and the last data items must have one of the following forms: 
- $\underline{\text { Variable name }}=$ constant, where the variable name may be either subscripted or not.

- Array name $=$ set of constants (separated by commas). The number of constants must be equal to the number of elements in the array and they must be in the same order. as the array is in storage, i.e., the first subscript chänges most rapidiy.

- Subscripted variable = set of constants (separated by commas). This form results in the set of constants being placed in consecutive array elements, starting with the element designated by the subscripted variable. The number of constants supplied must be equal to or less than the number of array elements between the given element and the last element in the array.

The NAMELIST variables retain their values throughout execution of the program and need not be respecified, with the following exceptions. Control integers LIB and LGLIB are set to zero prior to the start of each case. Also, when DRF values are to be read from a data library, the distance values of the library replace values submitted for $D$. It is important to remember that XIQ values submitted by card input will not correspond to distance values used by the program (unless values submitted as.D are the same as those in the DRF library). 
APPENDIX D

SAMPLE PROBLEMS 
APPENDIX D

\section{SAMPLE PROBLEMS}

Two sample problems are presented to illustrate preparation of input cards for BIVAR and SUBDOSA. No input cards àre needed for program BELI.

The first sample problem is for calculation of dose rate factors using program BIVAR. Dose rate factors are calculated for Pasquill Type $F$ and Hanford moderately stable meteorological conditions, a wind speed of $1 \mathrm{~m} / \mathrm{sec}$, a ground level release and downwind distances of from 100 to $10^{5} \mathrm{~m}$. . Input cards for this problem are shown in Figure D-1. A listing of the output. cards produced by BIVAR is given in Figure $D-2$.

NOTES:

- The first output card gives (in order) the release height, number of stabilities (MET), number of wind speeds (NUBAR), number of distances (NR) and the number of photon energy groups (GROUPS):

- The remainder of the output cards give dose rate factors, two cards per downwind distance.

A listing of the printed output is given in Figure D-3. NOTES:

- Included in the output listing are values used for $\sigma_{y}(S Y), \sigma_{z}(S Z)$, and $x / \eta$ for each $X$-grid integration position.

- The dose rate factors are the last 12 numbers of the 1 ine beginning with $D=$.

The second sample problem illustrates use of SUBDOSA to calculate gamma/ beta skin and eye dose for two release types. The first release is 1 curie of ${ }^{85} \mathrm{Kr}$ released at ground level under Pasquili Type $\mathrm{F}$ stability conditions, wind speed of $1 \mathrm{~m} / \mathrm{sec}$, and for a puff release (RT( 1$)=1 \mathrm{sec})$. The doses are calculated for the standard 10 downwind distances from 100 to $10^{5} \mathrm{II}$. The second calculation is for release of various quantities of four noble 
gas nuclides in three release periods: Both calculations are to be performed in the same run. Input cards are listed in Figure D-4.

NOTES:

- The first card is the title card for release of 1 curie ${ }^{85} \mathrm{Kr}$.

- The next three cards are the NAMELIST cards for this calculation.

- The parameters LIB and LGLIB are set positive to cause the main data libraries to be read from appropriate files.

- $L U L I B=1$ and LFDRF = 0 (not specified) indicating gamma dose rate factors are to be read from input file DRFLIB (in this example Library BIVLIB is assigned to file DRFLIB through an "ATTACH" CYBER control card).

- LBETA, LGAMA, and LCHIOQ are set positive to cause calculation of beta and gamma doses and normalized air concentration.

- NREL $=1$ and $R(1)=1$ indicate one release period of $1 \mathrm{sec}$ duration (puff release) is desired.

- $N R=10$ indicates that 10 downwind distances are to be considered. Values of $U$ need not be supplied when dose rate factors are read from DRFLIB since distance values are supplied with the dose rate factors. in tact, distance values submitted in NAMELIST would bc ovcrridden by the DRFLIB value.

- $\quad \mathrm{ITZ}=2$ indicates that nuclide decay during transit only will be considered.

- The parameters $N B D=2$ and $\operatorname{BDEP}(1)=7 .$, 100. indicate that two beta tissue depth dose calculations are desired (at 7 and $100 \mathrm{mg} / \mathrm{cm}^{2}$ ).

- $N G D=2$ and $\operatorname{TDEP}(1)=0 ., 5$. indicate that two qamma tissue depth dose calculations are desired (at 0 and $5 \mathrm{~cm}$ tissue depth).

- $\operatorname{NBDI}(1)=2,4$ indicates that the effective beta energy data are to be taken from the second and fourth position of the effective beta energy library (RNDBET, Appendix A). 
- The parameters $\mathrm{NBE}=2$ and: $\mathrm{NGE}:=1$. indicate that the eye dose is to be the sum of the second beta tissue depth dose $\left(100 \mathrm{mg} / \mathrm{cm}^{2}\right)$ and the first gamma tissue depth dose $(0 \mathrm{~cm})$.

- Similarly NBS = 1 and NGS = 1 indicate that the skin-dose is to be the sum of the first beta tissue depth dose $\left(7 \mathrm{mg} / \mathrm{cm}^{2}\right)$ and the first gamma tissue depth dose $(0 \mathrm{~cm})$.

- LDRFP $=1$ causes printing of gamma and beta dose rate factors.

- $\operatorname{NDRF}(1)=1$ indicates that the first set of dose rate factors on file DRFLIB are to be used for the gamma dose calculation.

- The next card is a title card for atmospheric stabilities (card Type 2).

- The next three cards specify 1 curie of ${ }^{85} \mathrm{Kr}$ for release in period 1 (card Types 4-6).

- The last card for this calculation (card Type 8) indicates that Pasquill Type $F$ stability will be used for the beta dose and normalized air concentration calculations and that the values of $\sigma_{y}$ and $\sigma_{z}$ are to be printed.

The second calculation follows with a title card and one NAMELIST card. The NAMELIST card includes values only for the parameters that are to be changed from the first calculation. (LIB and LGLIB are set to zero before each case to avoid unnecessary rereading of data libraries.)

NOTES :

- NEXT $=2$ to allow change of all variables but no 1 lbrary redus.

- Parameters NREL $=3$ and $\mathrm{RT}(1)=1800 ., 27000$., 57600. specify three release periods are to be considered of duration $30 \mathrm{~min}, 7.5 \mathrm{hr}$, and $16 \mathrm{hr}$. This corresponds to release over 1 day in three periods 0 $1.2 \mathrm{hr}, 1 / 2-8 \mathrm{hr}$, and $8-24 \mathrm{hr}$.

- $I T Z=1$ indicates that decay will be calculated from time zero to the start of each release period and during transit. 
- $\operatorname{NDRF}(1)=1,1,3$ indicates Pasquill Type $F$ dose rate factors will be used for the first two release periods and Pasquil1. Type $D$ for the third period.

- The next card (Type 2) gives the stability titles for each release period.

- Next follow three sets of inventory cards (Types 4-6), one set for each period. The curies of each nuclide represent the time integral of release rate with decay accounted for during release only (because ITZ $=1$ ).

- The last three cards indicate the stability type to be used for the beta dose and normalized air concentration calculations and also cause printing of $\sigma_{y}$ and $\sigma_{z}$ values used.

Output for the second sample problem is shown in Figure D-5. The output is self-explanatory; the execution time is printed for each case. The first case took considerably longer than the second case because the three main data libraries were read during the first case. 
FIGURE D-1. First Sample Problem Input

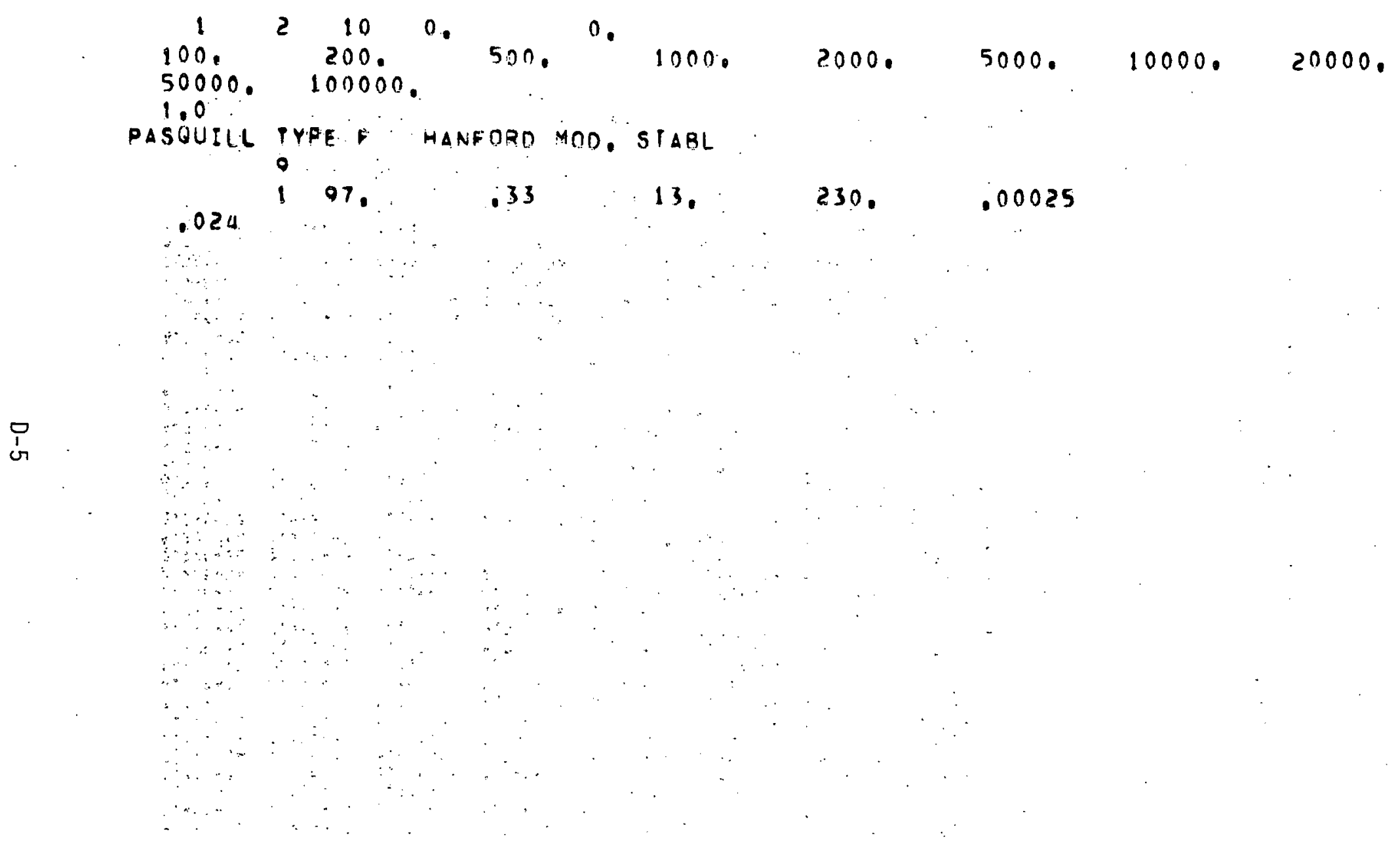




\section{FIGURE D-2. First Sample Problem Card Output}

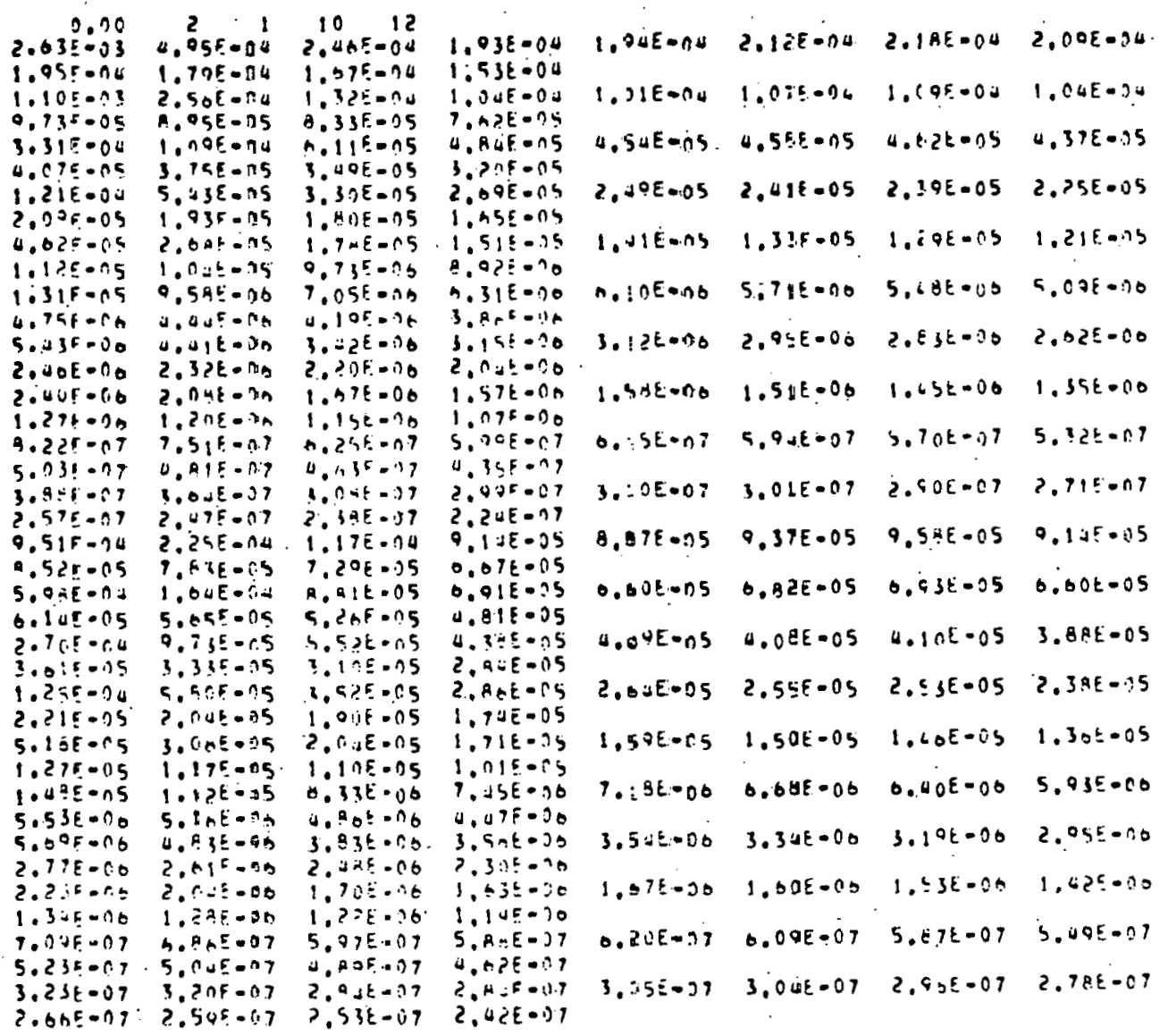


FIGURE D-3. First Sample Problem Output

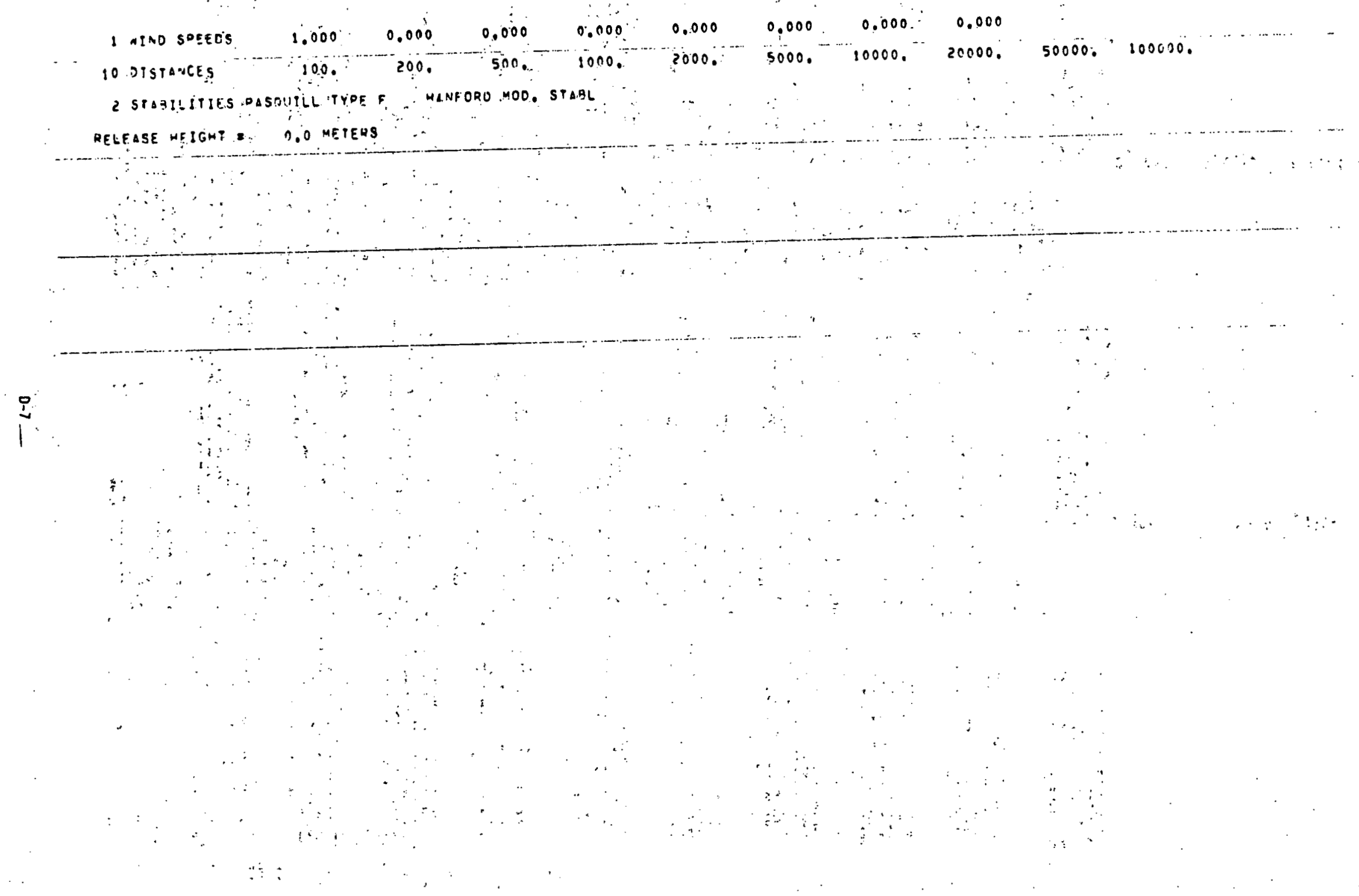


sin

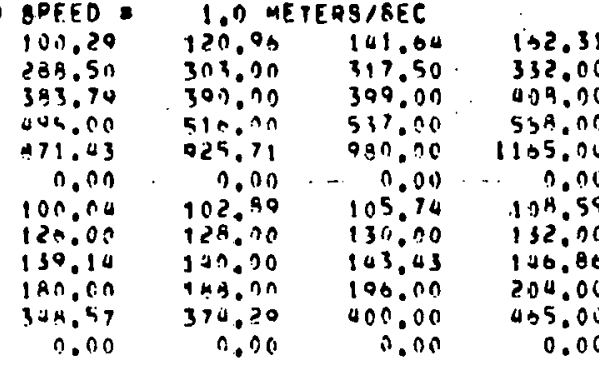

182.09
340.50
417.00
579.00
1350.00
0.00
111.05
134.00
150.20
212.00
530.00
0.00
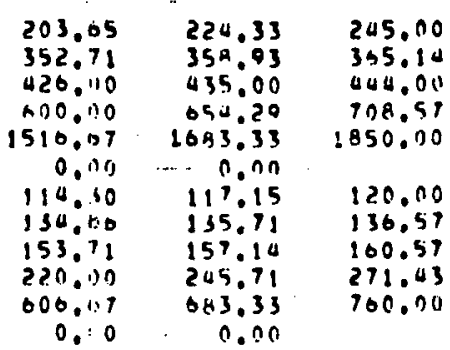

250.50

371.30
453.00

162.86

2025.100

274.00

377.57

37.00

122.00

137,43

2200.00

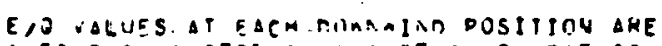

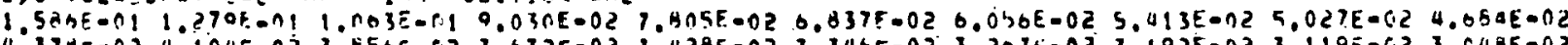
1. 2.9450 (a)

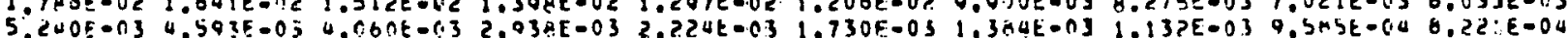

$D=100, Y^{0}=$

100.27
300.20

110.00
100.00

Q10 0.20

1513.07

$52=100.04$

137.14

27.00

317.71
$36 ? .00$

$30 ? .00$
00.09

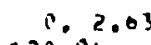

$10.2 .03 t-032.05 E-012.40 \mathrm{~K}-04$

$\begin{array}{ccc}120.00 & 141.64 & 102.31 \\ 420.20 & 940.00 & 540.00\end{array}$

$1.03 E=04 \quad 1025=04$

182.98
600.00
757.43
838.80
085.71
200.00
111.45
220.00
204.57
333.14
837.14

203.35
619.30
165.37
84.790
1138.37
2375.90
114.30
229.30
298.13
357.010
355.71
940.30

$2,12 E-144$
224.33

224.33
639.00
773.71
065.00

$18 E-0$

$2.09 \varepsilon-0<1.058$

$245.00 \quad 286.43 \quad 327.80$

$\$ 57.00 \quad 070.00 \quad 005.00$

$\begin{array}{lll}741.85 & 790.00 & 794.14 \\ 405.00 & 944.00 & 223.00\end{array}$

914.43 aर2.5?

1035.86

$083.33 \quad 1959.00 \quad 2025.00$

$102.99 \quad 105.74 \quad 108.5^{\circ}$

$151.03 \quad 174.29$

$321.57 \quad 335.43$

301.20
$453: 13$

red.0

197.14

320.29

050.00

ABO.00
1207.16

c550.00

c550.00

117.15

30.000
302.50

300.00

1000.00
$120.00 \quad 125.7: \cdot 131.43$

$306.14 \quad 350.00 \quad 205.00$

$\begin{array}{lll}306.14 & 310.00 & 313.85 \\ 355.00 & 364.00 & 373.00\end{array}$

$492.40 \quad 511.43 \quad 530.00$

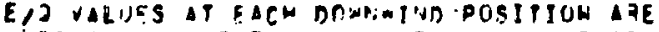

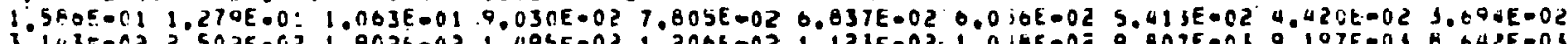

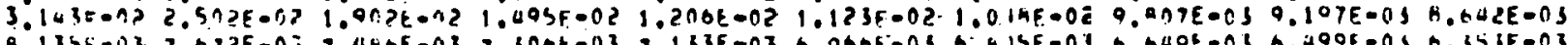

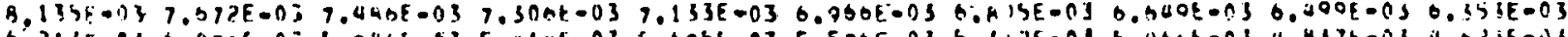

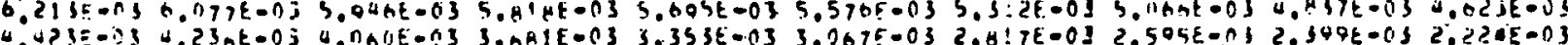

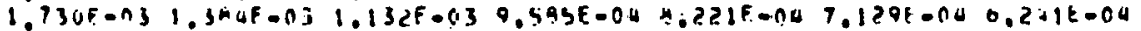

Sy.

100.20
1090.86
1983.33
1005.00
1000.09

$52=$

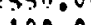

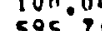

127.3

705.14

inco.on

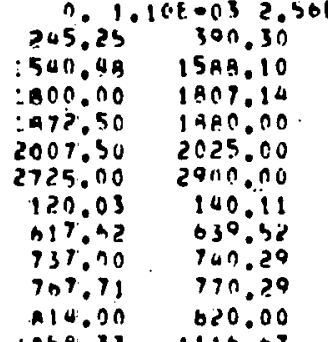

6n 1.22

700:10

700.16
683.33

1817.20

1887.50

3075.00

3520

220.10

743.57

777.06

831.14
1115.00

तb8.33 11:0.07

NE

1205.00

3250.00

$310, \mathrm{C}$

TR3.3 3

740.80
775.43

1233.33

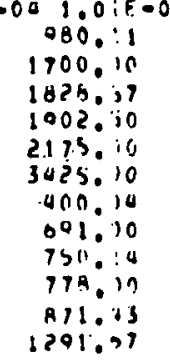

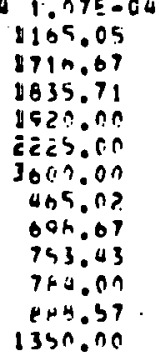

$1.00 t-041$

. OUE-04

$0.73 E=05 \quad 0.95 E-05 \quad 8.33 E-05 \quad 9.0 .2 E=0.5$

133.35

1750.00

1750.00
1350.00

0.37 .5111955 .00

2275.00

23?5. 60

530.00

700.33

756.71

551.00

724.00

760.0

146.00

922.00

1700.09

1072.50

2375.000

573.61

721.67

802.00

940.00

90.00

$79 E-04 \quad 1.07 t=04 \quad 1.53 t-04$ 
$1.540 E-01 \quad 5.406 E-02 \quad 2.910 E-02 \quad 1.205 E-02 \quad 0.496 E-03 \quad 4.059 E-032.038 E-03 \quad 2.224 E-03 \quad 2.003 E-03 \quad 1.919 E-03$ $1.790 E-03 \quad 1.673 E-03 \quad 1.5 H 7 E-03 \quad 1.471 E-03 \quad 1.384 E-03 \quad 1.355 E-03 \quad 1.2 .27 E-03 \quad 1.30 C E=03 \quad 1.274 E-03 \quad 1.248 E-03$

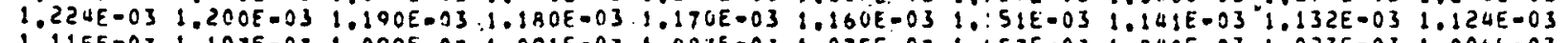
$1,115 E-031.107 E-03 \quad 1.09 Q E-03 \quad 1.091 E-03 \quad 1.083 E-03 \quad 1.075 E-031.0357 E-03 \quad 1.040 E=03 \quad 1.023 E-03 \quad 1.000 E-03$

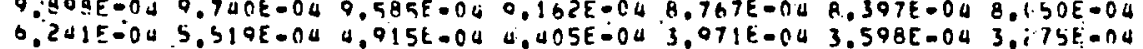

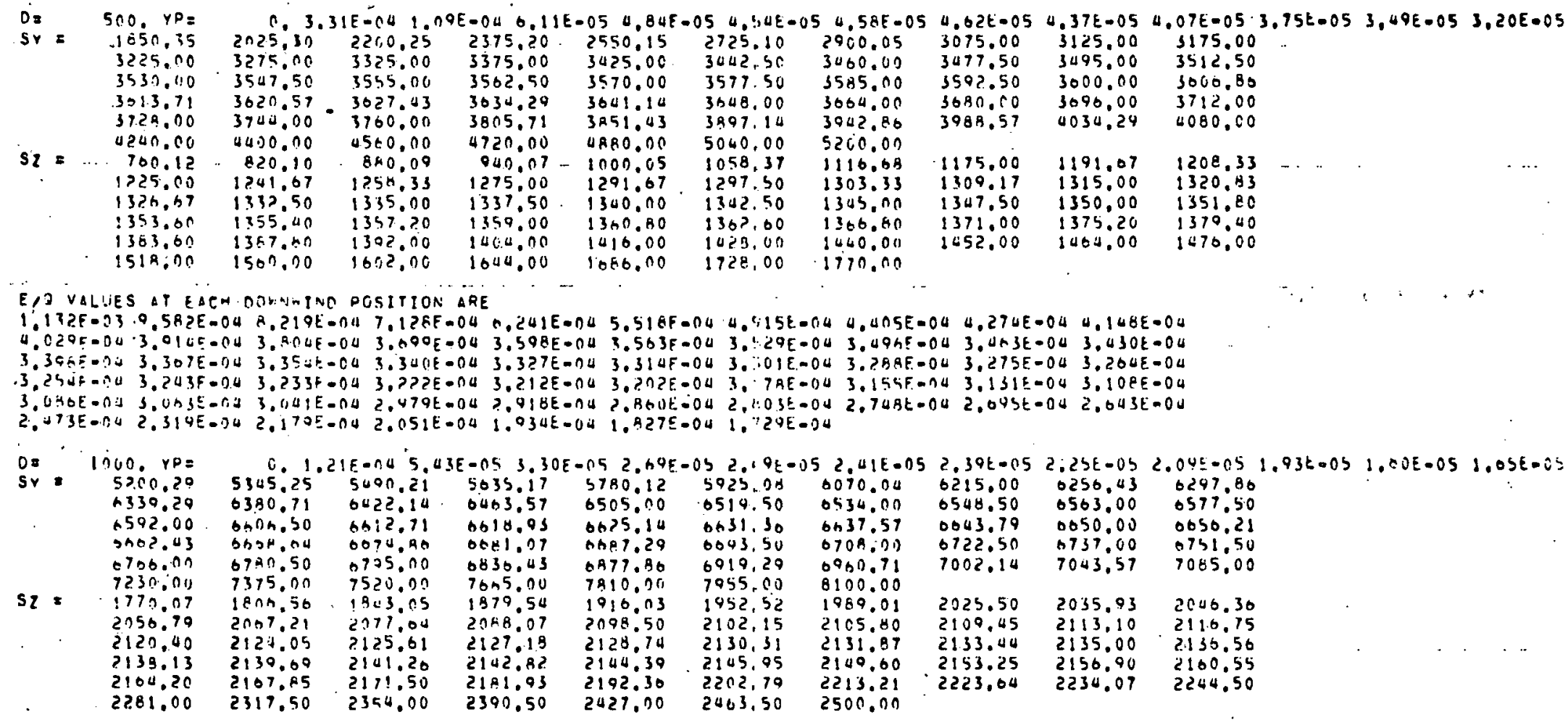
E,O YALUES AT EACH DOWVITND ROSTTIOIN ARE

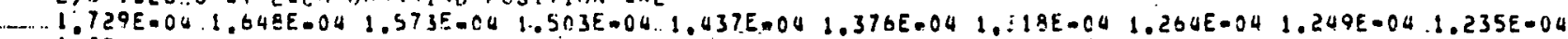

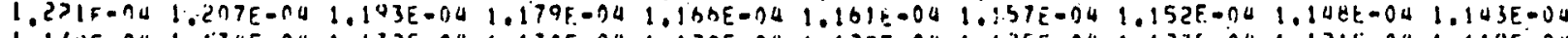
$1.13 U E-n 4$.

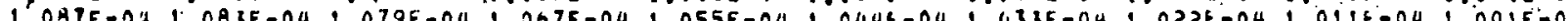
$0.651 E-059.312 E-058.991 E-05$ 8.686E-05 8.39.7E-05 8.121E-1.5 7, t:OOE-05:

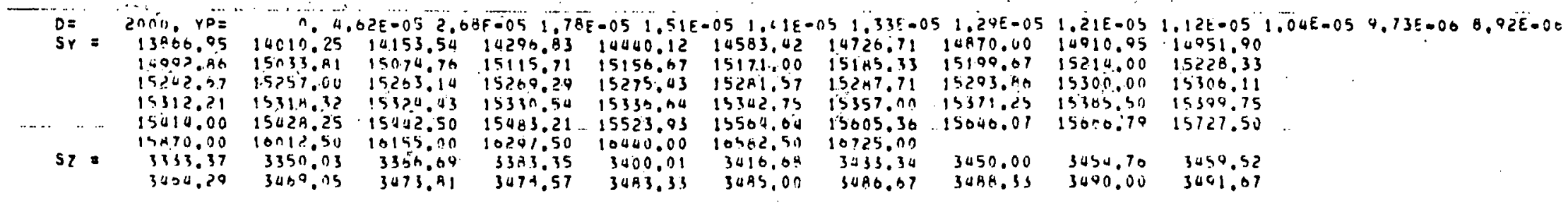

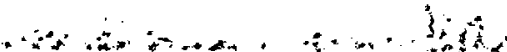




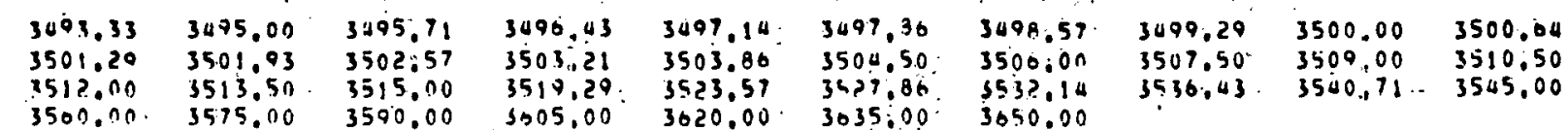

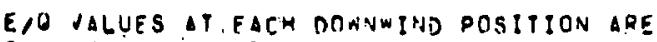

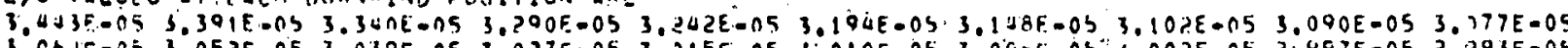

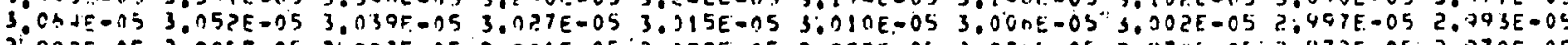

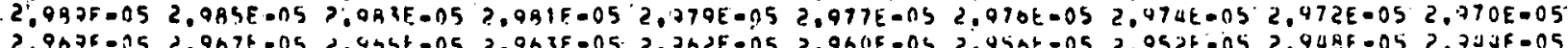

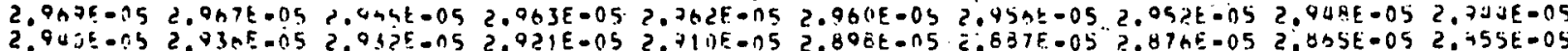

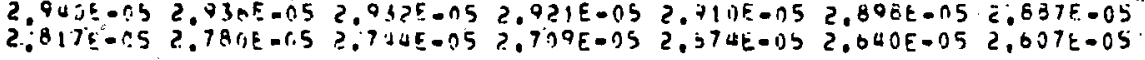

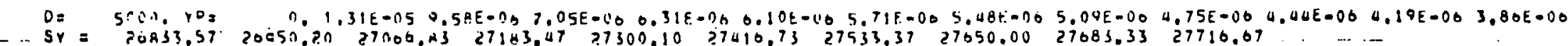

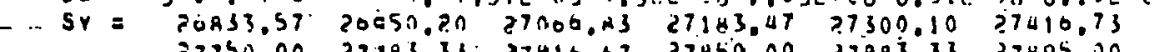

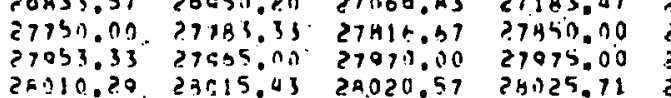

$27053.33 \quad 27655.0127079 .00 \quad 27075.0027000 .00$

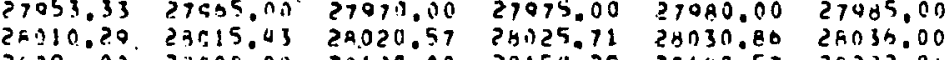

$s 2=$

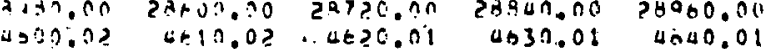

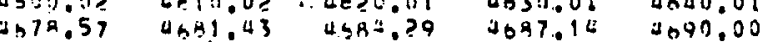
4590.00 4697.0n 4507.43 4697.80 4608.29 $4700.09 \quad 4511.03 \quad 4111.37 \quad 4701.714792 .06$

472.57

$474 \mathrm{H.OO}$

$75 t: 00$

4764.00 27000.00

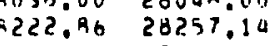
$27650.00 \quad 27085.33 \quad 27710.67$ $\begin{array}{lll}27419.35 & 27930.00 & 27941.67 \\ 27995.00 & 26000.00 & 26005.14\end{array}$ $27995.0026000 .0025005,14$. 28000.04 28072.00 2no84.00

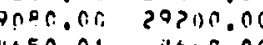
$4501.00 \quad 460.000$ 4hat. 4698.11 $469 \cos ^{-0}$ $4670.00 \quad 4073.80 \quad 4075.71$ $4693.00 \quad 4694.00000909 .00$ 472.00 (47.00 472.80

E, VILUES AT EACH ROWNATVD PDSITIUNN ARE

1.2-75-115 $1.2 B 1 E-05 \quad 1.273 E-051,265 E-C 5 \quad 1.256 E-05 \quad 1.24 A E-05 \quad 1.240 E-05 \quad 1.233 E-05 \quad 1.230 E-05 \quad 1.226 E-05$ $1.270=-051.2245-051.22115-051.214 E-051.217 E-05$ 1.210E-05 1.215E-05 1.21SE-05 1.21UE-OS 1.2113E-0S

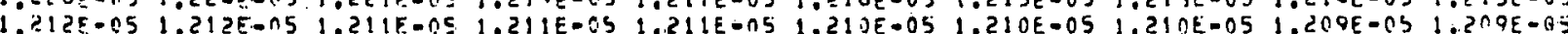

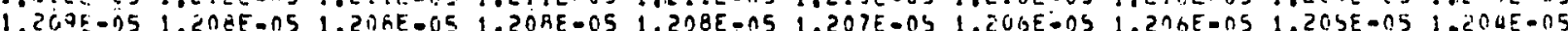

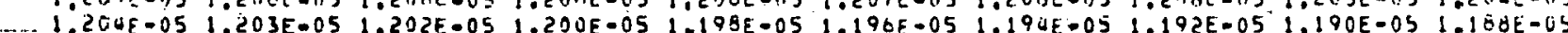

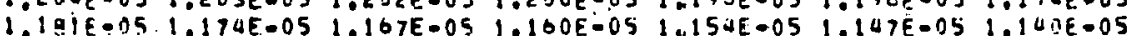

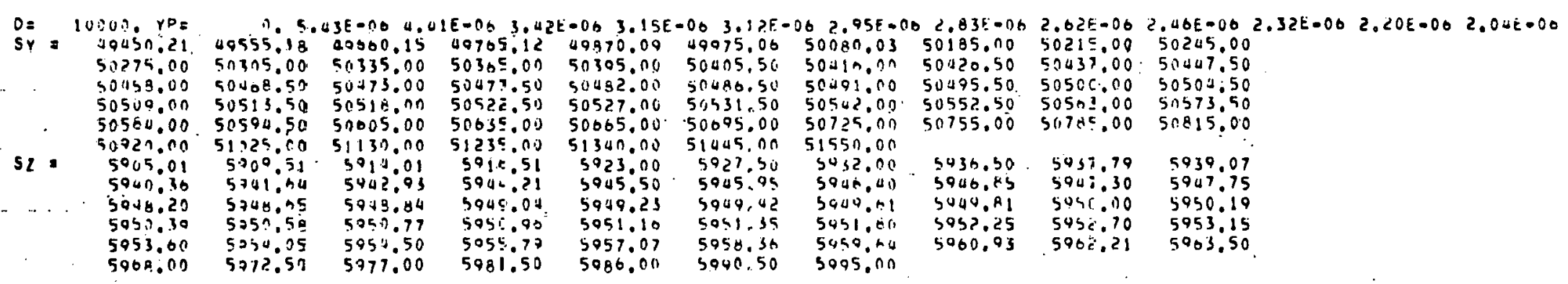

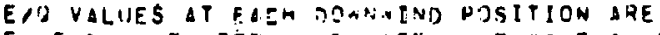

$5.4505-005.435 E-365.4164-005.404 E-005.388 \equiv-00 \quad 5.373 E-065.557 E-005.343 E-005.3345 .005 .333 E-00$

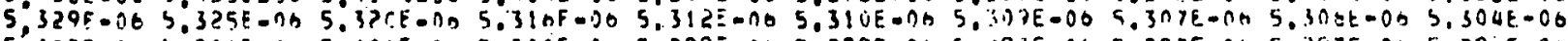

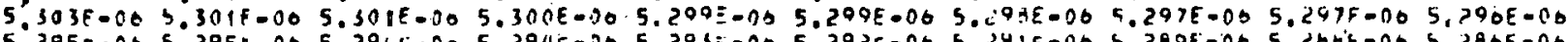

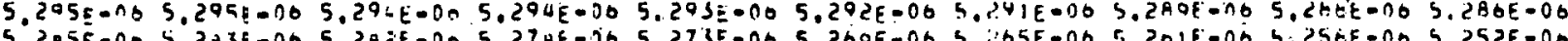

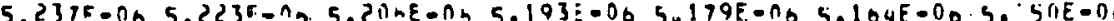

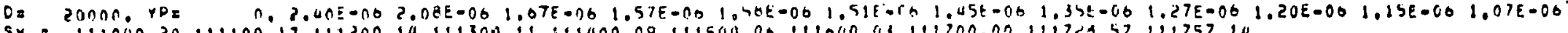

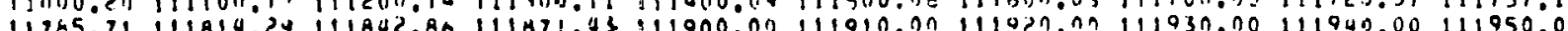


$111960.00 \quad 1119.70 .150 \quad 111979.29 \quad 111979.57111982 .86 \quad 111987.14 \quad 111091.43 \quad 111995.71112000 .00112003 .86$ 112009.71112011 .59112015 .43112019 .29112023 .14112027 .001112036 .001112045 .00112054 .00182003 .000

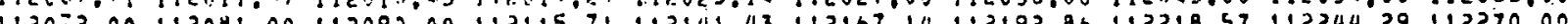

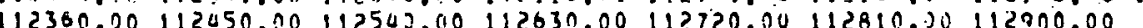

sz:

\begin{tabular}{|c|c|c|c|c|c|c|c|c|c|}
\hline & & & & & & & & & \\
\hline & & & • & 10 & & 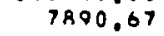 & $7 \mathrm{HQ3}$ & 7803.07 & 7894 \\
\hline & & $a b$. & 7897.00 & 7807.07 & 7.30 & 78 & 7 & 00 & 3 \\
\hline 20 & 729 & 7897 & $7899: 50$ & $7 \times 99.60$ & 7809,70 & 7800 & 78 & 7900.00 & 900.08 \\
\hline $\begin{array}{l}0 \% 0.15 \\
041.40\end{array}$ & $\begin{array}{l}70110.23 \\
7001.55\end{array}$ & $\begin{array}{l}700.30 \\
7001:-5\end{array}$ & $\begin{array}{l}7900.37 \\
7002.25\end{array}$ & $\begin{array}{l}7000.45 \\
1002.75\end{array}$ & $\begin{array}{l}7000.33 \\
7903.25\end{array}$ & $\begin{array}{l}7900.70 \\
7403.75\end{array}$ & $\begin{array}{r}7900.88 \\
7904.25\end{array}$ & $\begin{array}{l}7901.05 \\
7904.75\end{array}$ & $\begin{array}{l}7901.23 \\
7905.25\end{array}$ \\
\hline
\end{tabular}

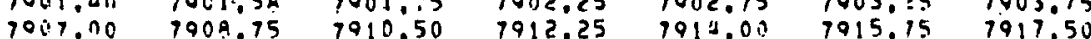

SA VALUES AT EACH DCAMEIUN DOSTT:OV AHE

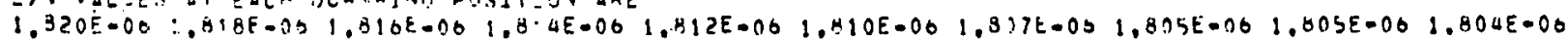

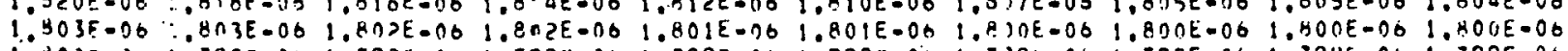

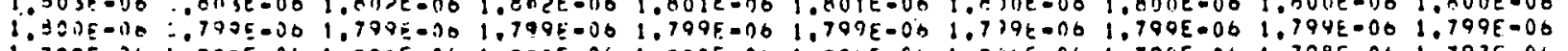

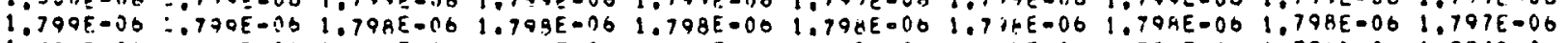

$1.797 E-106 \quad: .797 E-06 \quad 1.777 E-06 \quad 1.700 E-00 \quad 1.790 E-00 \quad 1.705 E-001.735 E-00 \quad 1.774 E-001.794 E-001.793 E-00$

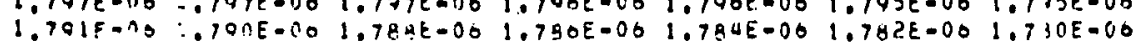

$D=\operatorname{sog} 90, Y H=$

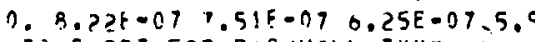

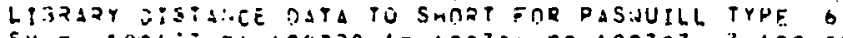

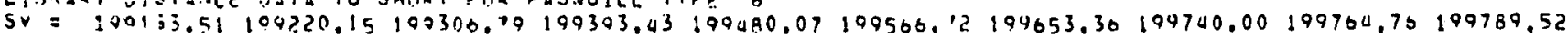

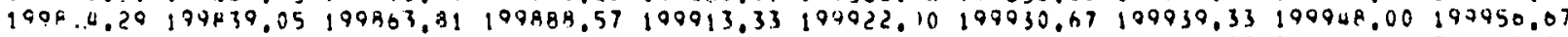
100005.33109094 .00109977 .11199991 .43102995 .1 . 199088.10197992 .57109096 .29200000 .00200000 .00

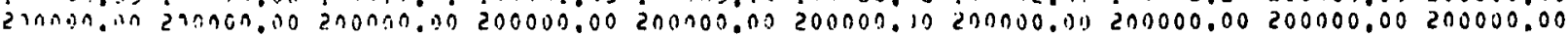

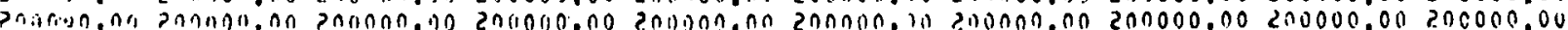

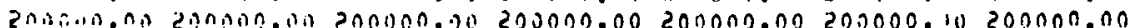

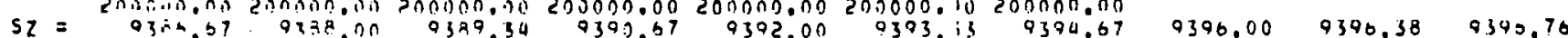
Q397:?" 9397,5

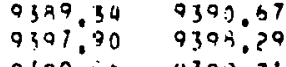

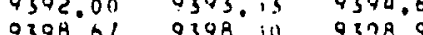
$03.00 .47 \quad 9309.00 \quad 0309.50 \quad 4304.71 \quad 0309.77 \quad 9399.13 \quad 4309.89$

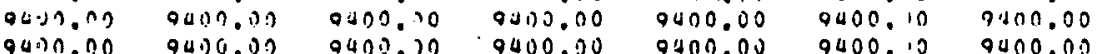
0.400 .00
$9309.07 \quad 9399.20 \quad 0309.33$
$9399.90 \quad 9400.00 \quad 040 \% .00$

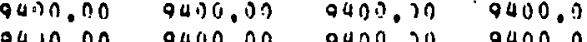

E/O VALUES AT EACH DCWMAINO EOSITION ARE

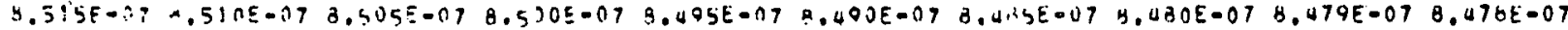

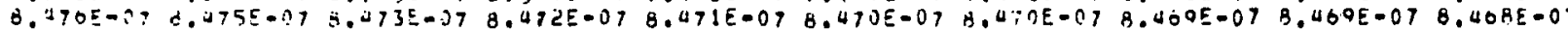

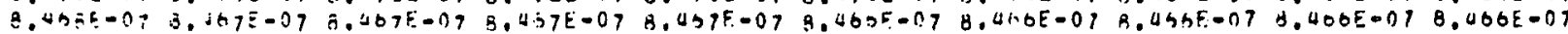

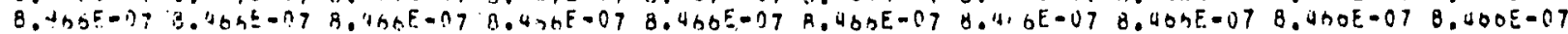

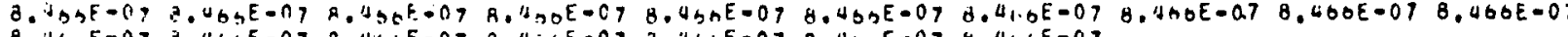

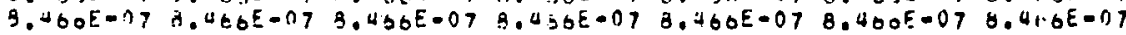

$0=100000, Y 0=0.3 .88 E-073.64 E-073.08 E-072.99 E-073.11 E-073.01 E-072.90 E-072.71 E-072.57 E-072.47 E-072.38 E-072.24 E-09$ 
DOSE RATE FACTORS FOR HANTORO MOD, STABL DATE OTIOTITS

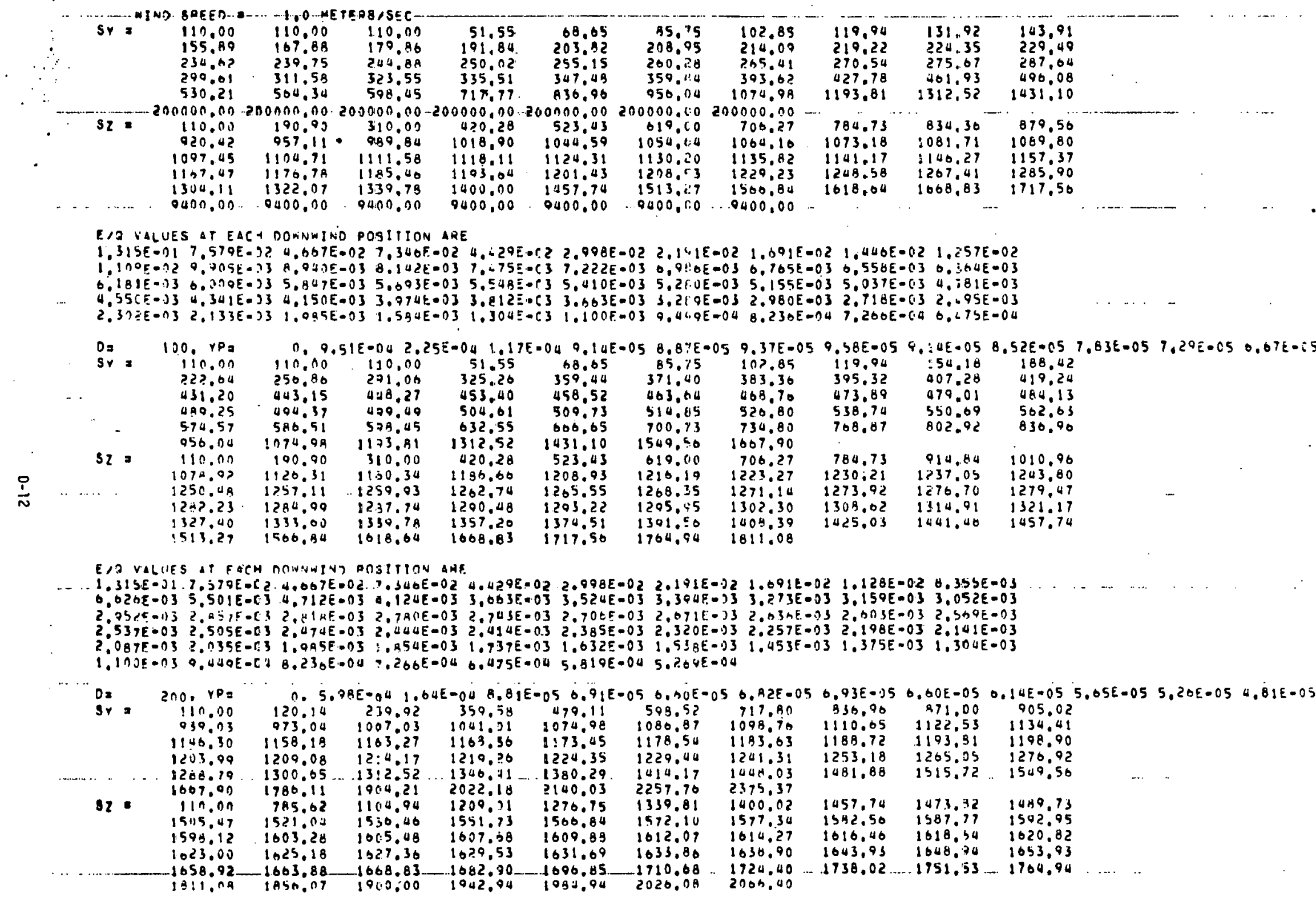

E/O VALUES AT EAC- OOWNWIND POSITION ARE 
$1.3: 5 E-01 \quad 1.0 Q 6 E-02 \quad 0.004 E-n 3 \quad 3.661 E-03 \quad 2.002 E-03 \quad 1.995 E-03 \quad 1.594 E-03 \quad 1.304 E-03 \quad 1.240 E-03 \quad 1.180 E-03$

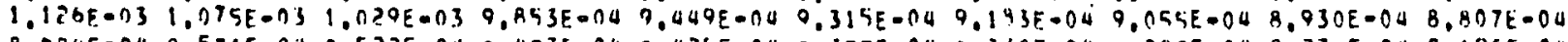

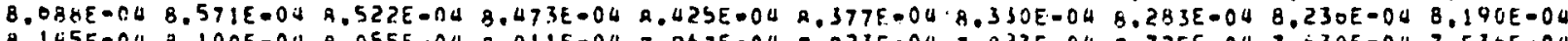

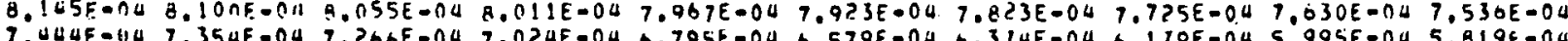

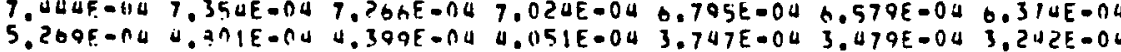

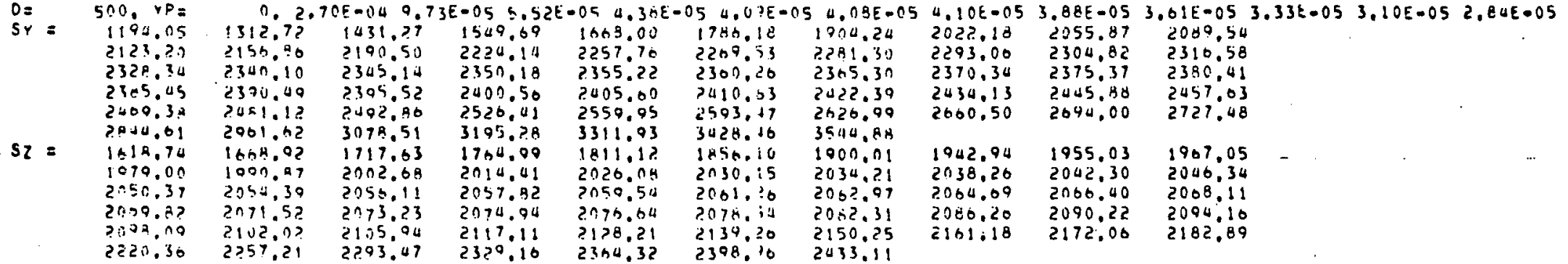

E, C VALUES AT FACH DONIAHINO POSITION ARE

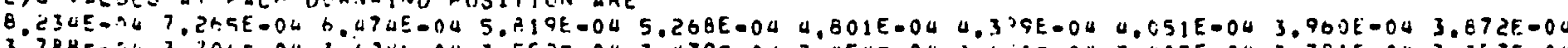

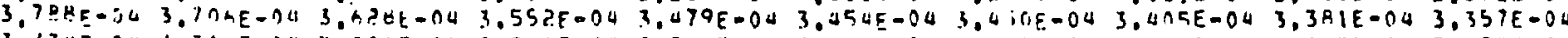

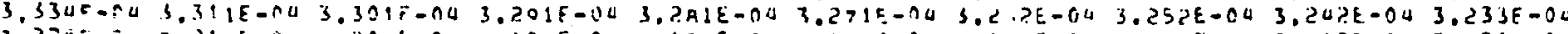
3.

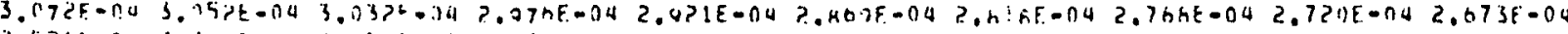

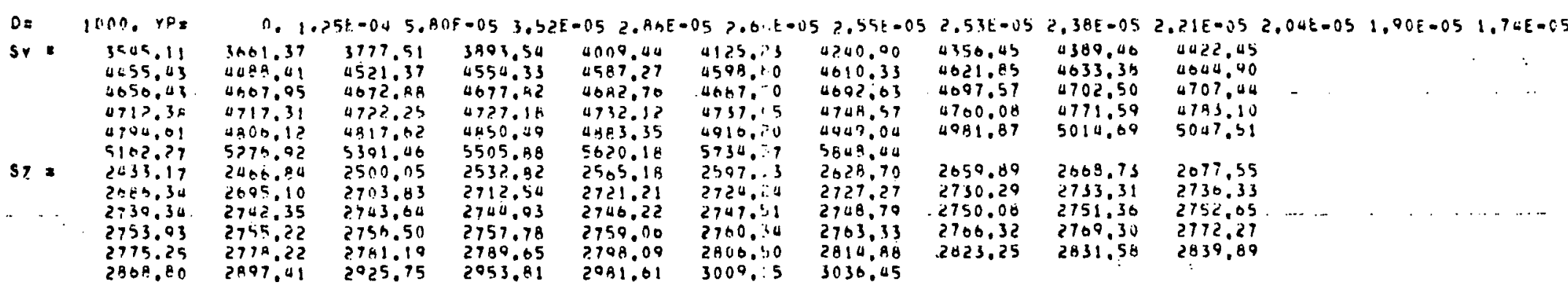

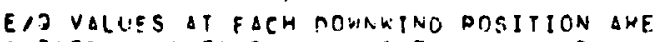

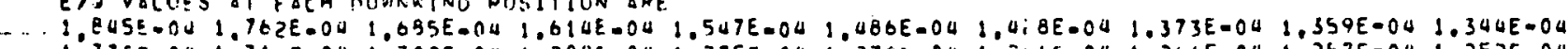

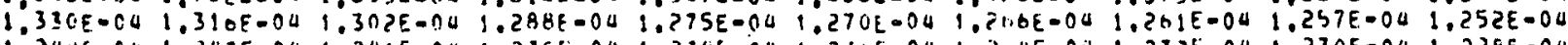

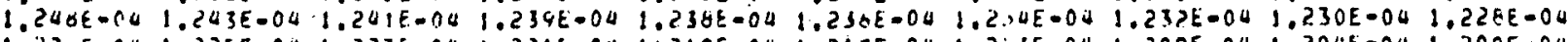

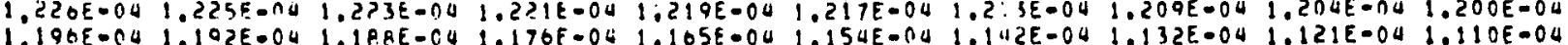

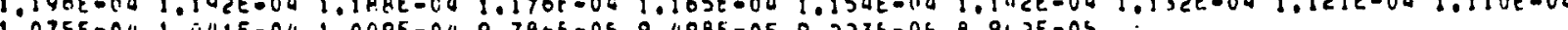

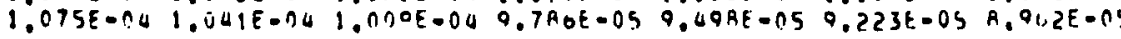

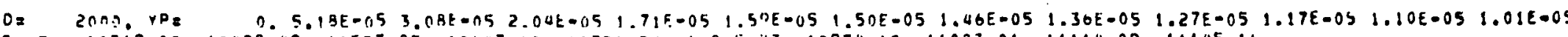

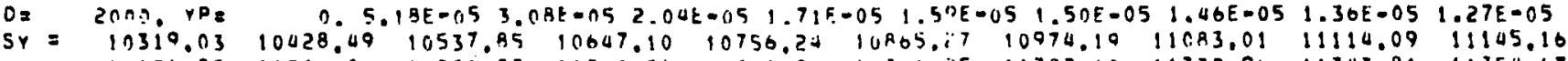
11311.0.5 $11322.10 \quad 11332.90 \quad 11343.81 \quad 11354.07$

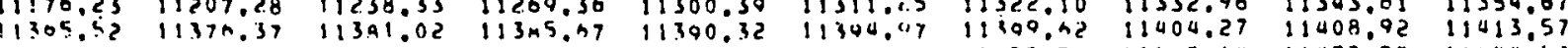
11418.?2 1102?.86 11427.51 11432.16 11436.A1 11141.60 1149?.311 114n3.14 11473.98 114k4.63 11005.40 11505.50 11517.34 11548.50 11570.25 11010.10 11041.1? 11672.64 11702.90 11733.87 $11+41.0911040 .07$ 172557.40 12845.04 12273.3? 12306.611 $124 \times 8.37$

4039.21 4059.57 4070.84 $0100.014180 .07 \quad 4125.74 \quad 4131.50$

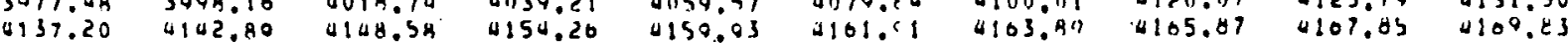




$\begin{array}{llllllllll}4171.81 & 4173.70 & 41.76 .63 & 4175.48 & 4176.33 & 4177.17 & 4178.02 & 4178.87 & 4179.71 & 4180.56 \\ 4181.40 & 4162.25 & 4133.10 & 4183.24 & 4184.79 & 4185.53 & 4187.60 & 4189.57 & 4191.54 & 4193.51 \\ 4195.47 & 4197.44 & 4199.40 & 4205.01 & 4210.62 & 4216.21 & 4271.80 & 4227.38 & 4232.75 & 4238.51 \\ 4257.93 & 4277.27 & 4276.51 & 4315.67 & 4334.74 & 4353.93 & 4372.64 & & & \end{array}$

E/O VALUES AT EACH DCWUNIVO POSITION ARE

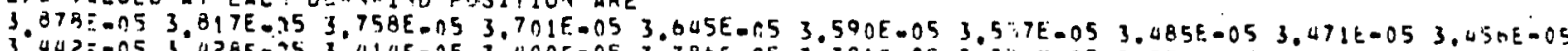

3.

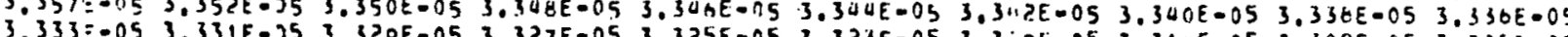

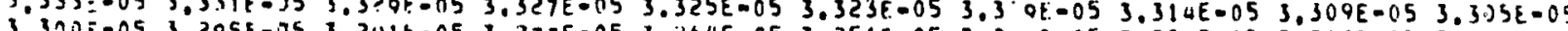

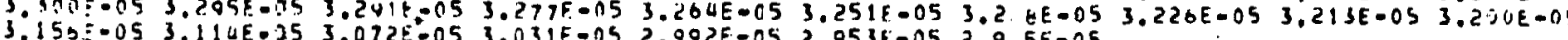

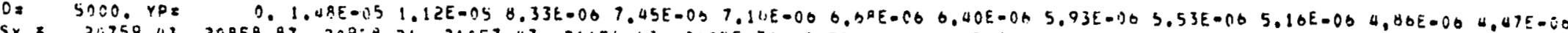

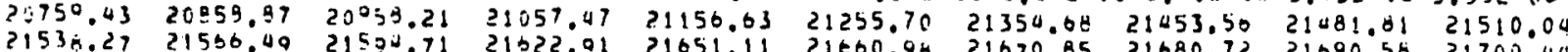
21535.27 21550.00 2150.71 21622.01 21651.11 21660.08 $21670.85 \quad 21680.72 \quad 21690.58 \quad 21700.40$

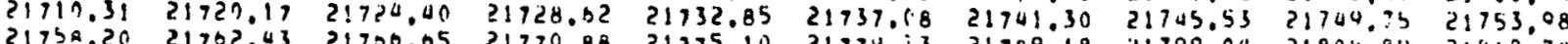

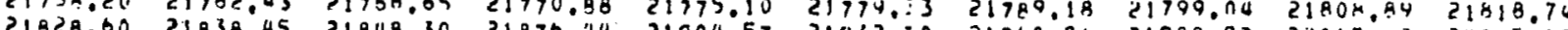
$21328.0321238 .4521949 .3021876 .4021904 .5721932 .0021960 .8121988 .02 \quad 22017.02 \quad 22045.12$

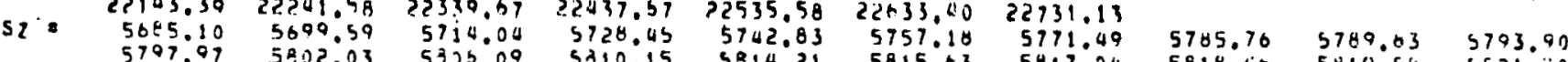

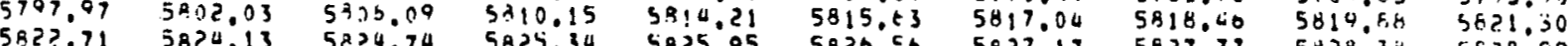
$58 ? .71 \quad 5824.13 \quad 5824.74 \quad 5825.34 \quad 5925.95 \quad 5826.56 \quad 5827.17 \quad 5827.79 \quad 5828.38 \quad 5829.00$

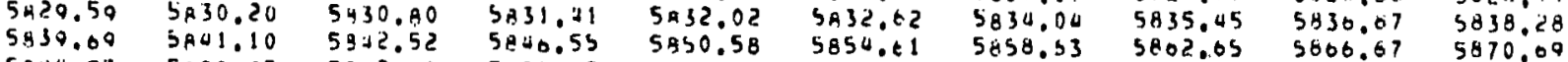
$5840.73 \quad 5898.73 \quad 5912.70 \quad 5926.03 \quad 5900.54 \quad 5054.41596 .25$

EIO VILUES AT EACH OUWVAIN: DODITION AKE

D $\quad 1.340 F-0.51 .330 E-051.320 E-05 \quad 1.319 E-05 \quad 1.310 E-05 \quad 1.301 E-051.291 E-05 \quad 1.28 ? E-05 \quad 1.200 E-05 \quad 1.277 E-05$

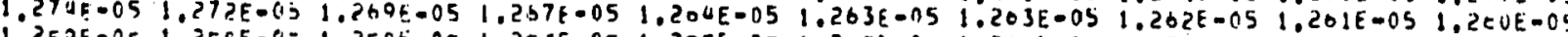

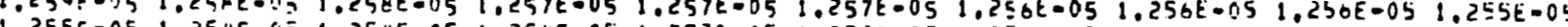

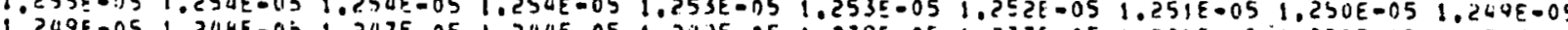
$1,22 E-05$.

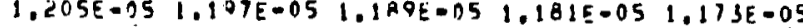

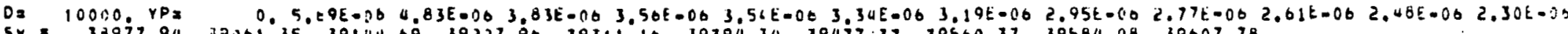
$371161.35 \quad 39144.00 \quad 30227.00 \quad 30311.10 \quad 39394.70 \quad 394773739560.3739584 .08 \quad 39607.78$ 37631.4A 30655.17 3967A.R5 30702.53 30776.21 39734.69 $39742.78 \quad 34751.00 \quad 34759.3439767 .63$ $30775.91 \quad 39784.1930797 .94 \quad 30701.29 \quad 30704.83 \quad 39798.3839801 .93 \quad 39805.4839809 .03 \quad 30812.58$ $\begin{array}{llllll}30930.31 & 30933.46 & 39842.14 & 39850.41 & 39858.69 & 39866.96 \\ 39939.03 & 30902.65 & 39946.27 & 0000.80 & 40035.06 & 40057.08\end{array}$

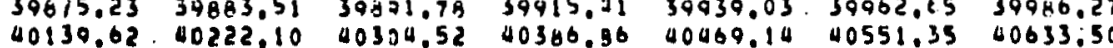
0130.62 . 0222,10 40334.52 40386.3640069 .1040551 .350633 .50

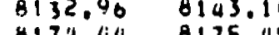
$8173.43 \quad 8174.6400$

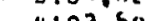

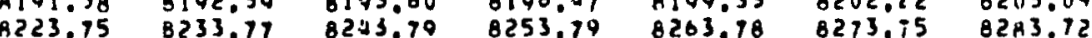

$8180.54 \quad 8187.55$

\subsection{8}

8153.22 tis6.11

8159,00
8178,48

8183.09 8183.52 8183.05

6189.378190 .57

-... E/O VALUES AT EACM DOWNININD. POSITION ARE

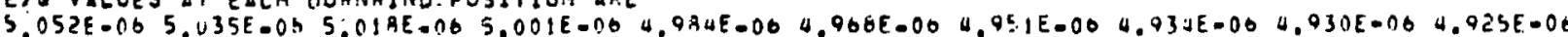
$4.052 E-065$.

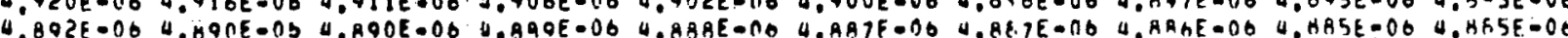

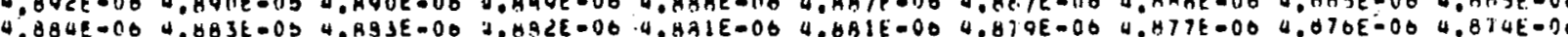

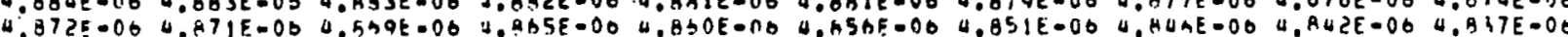
$4.821 E-00 \quad 4.800 E-06.4 .790 E=06 \quad 4.774 E=06 \quad 4.759 E-00 \quad 4.744 E-00 \quad 4,7 \dot{\prime}^{\prime} 8 E-C 6$;

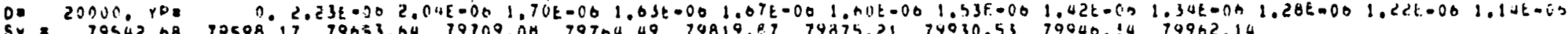

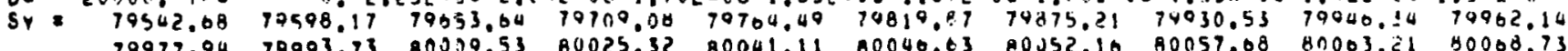

(... 


\begin{tabular}{|c|c|c|c|c|c|c|c|c|c|}
\hline $\begin{array}{l}99.26 \\
101.08 \\
1140.52\end{array}$ & $\begin{array}{l}80079.79 \\
60103.45 \\
80146,04\end{array}$ & $\begin{array}{l}\text { ACOR?. } 15 \\
10105.82 \\
30151.59\end{array}$ & $\begin{array}{l}\text { BUOAU.51 } \\
80108.18 \\
80167.33\end{array}$ & $\begin{array}{l}A 00 A B . A B \\
20110.55 \\
801 A 3.10\end{array}$ & $\begin{array}{l}80080.25 \\
80112.92 \\
80198.56\end{array}$ & $\begin{array}{l}80091.62 \\
80118.44 \\
80214.53\end{array}$ & $\begin{array}{l}98 \\
96 \\
39\end{array}$ & $\begin{array}{l}80096,35 \\
80129.40 \\
80246.14\end{array}$ & $\begin{array}{l}80098.72 \\
80155.00 \\
80251.90\end{array}$ \\
\hline 317 & 803 & 20127.13 & & 805 & 2.19 & & & & \\
\hline $\begin{array}{l}29: \\
250\end{array}$ & & & & & & & & & \\
\hline & & 12 & & & & & & & \\
\hline 238 & 12 & 120 & 12 & 94. & 16 & 1288 & 12 & 1 & 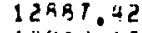 \\
\hline & 12804 & 1234 & 12291.18 & 12802 & 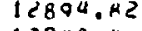 & $\because$ & $12494.4 \mathrm{H}$ & 1290 & 124 \\
\hline
\end{tabular}

E/O VALUES AT EACN DOW'JWIND SOSIIJON DRE

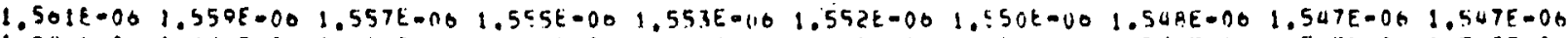

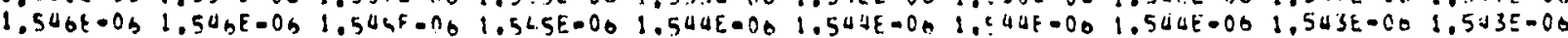

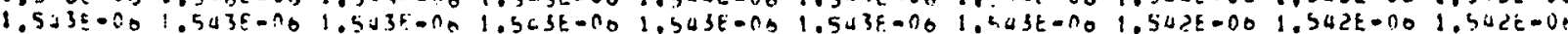

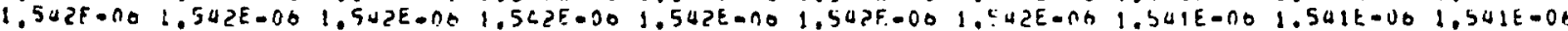

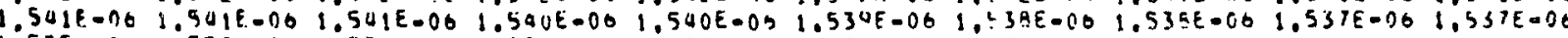

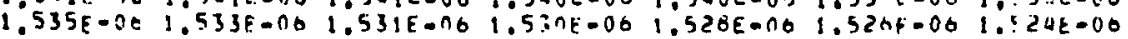

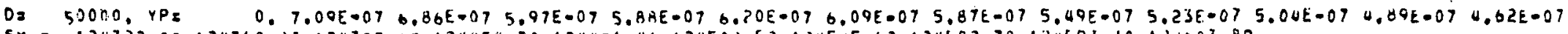

Sr $=134322.29174300 .113174307 .17124434 .30124471 .41124504 .52124545 .62124582 .70124593,30124303.89$

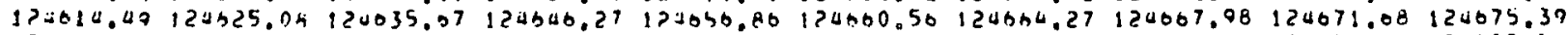

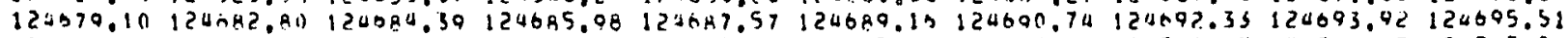

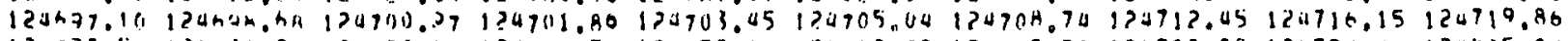
$120.233 .50124727 .27124730 .77 \quad 124701.50124752 .14124742 .72124773 .3 n 124783.89124794 .40124605 .00$ $123842.06 \quad 124879.07 \quad 124916.37 \quad 124953.06 \quad 170990.03125027 .00 \quad 123063.95$

ENO VALUES A TACM DOWNAIND POSITIUN DRE

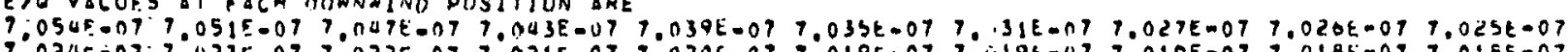

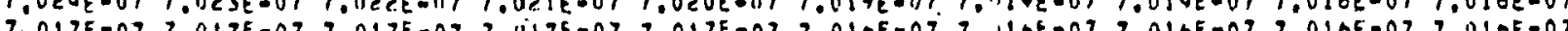

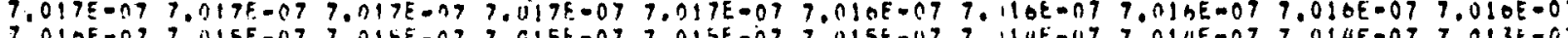

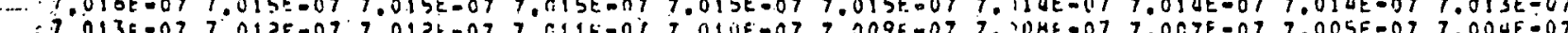

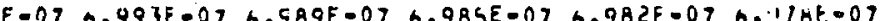
De $10003 n$. YP.
$0.3 .23 E-0173.20 E-072.84 E-072.84 E-073.35 E-07$
$0.04 E=072.96 E-072.78 E-07 \quad 2.60 E-07 \quad 2.59 E-07 \quad 2.53 E-072.42 E-07$ $\because y$ 
FIGUFE D-4. Second Sample Problem. Input

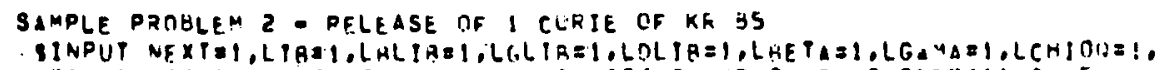

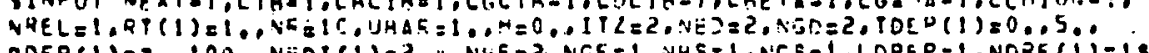

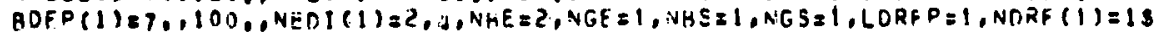

PASOUILLF

ONE CUPDE OF KA BS

Ko

851.0

SI YPLE PRCIRLEM 2 - QELEASE IN 3 PENIODS

SINPIT

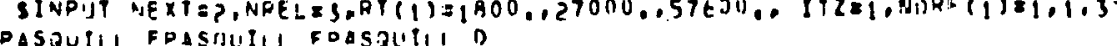

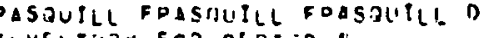

$\sin (x)$

$1.2 \times E[33$

$2.0 \times 133$.

1.0

TUVEYTIHY BOR DERI:O

as

$18, \times 5133$

30. XE 135

15.

TUVENTJRY FOR PER?

Ko

57.6x 85

$38.4 \times E \quad 1.33$

$04.0 \times 2135$

32.0 33 
SAMPLE PROBLEM 2 - RELEASE OF 1 CURIE OF KR BS

\section{ODSE RATE FACIORS}

STABILITY DASBUILL F RELFASE PERTOD

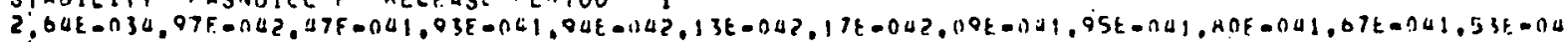

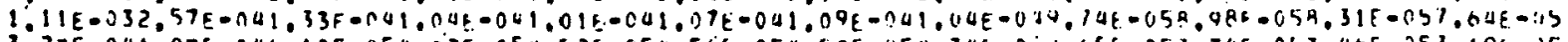

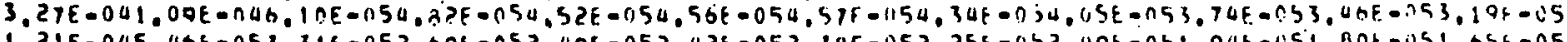

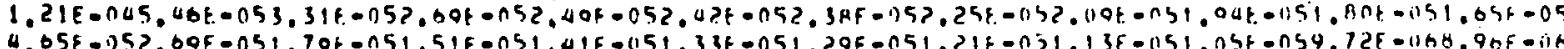

1 S2E-050. OBE-11G7.

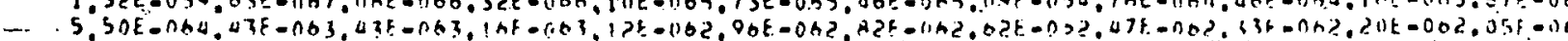

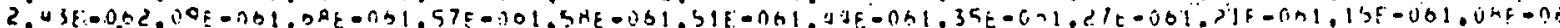

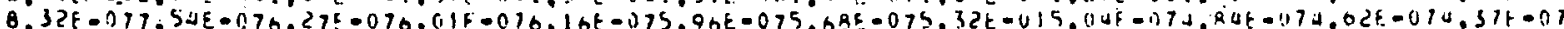

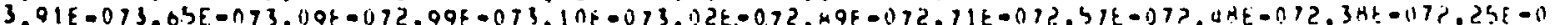

ONE CIIRTE OF KQ RS

NUCLIDF. IVUFWIORY IN CIIRIES FOR GELEASH TIME I

$X^{R}$ SS $1, n i j E+0$

SIGMA YNO Z FOR STAMTLITY i

DISTANCE FHUM RELFASEITITT NETERS

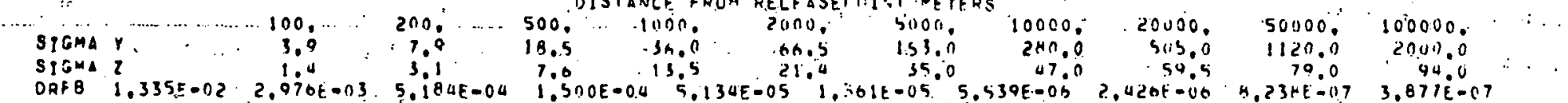


SAYPLF POCHLEM ? - RELEASE OF I CURTE UF KR Bל

DATE $08 / 12115$

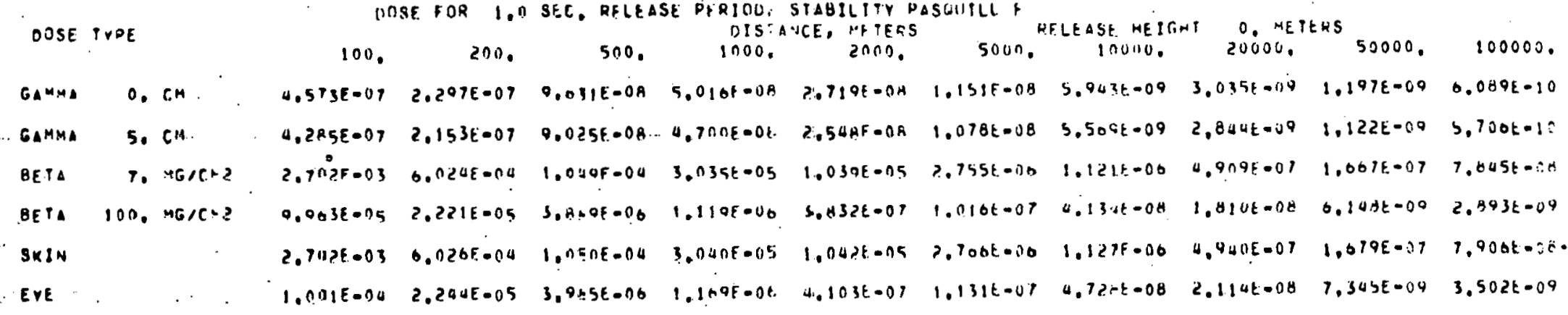

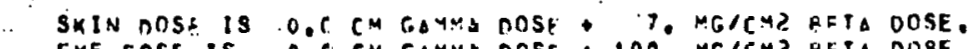

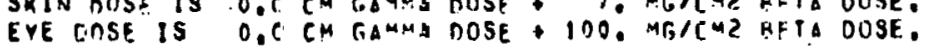

SAMPLE PREgLEM 2 - QELEALE OF I CURTE OF K.O 85

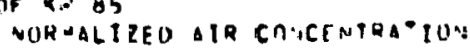

PEQION SIAATLITY

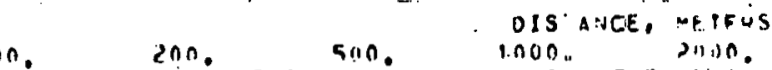

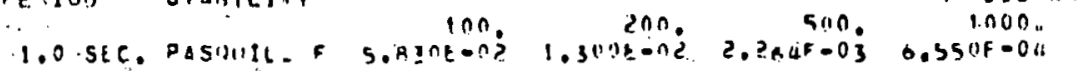
$\because$ a $+\cdots$

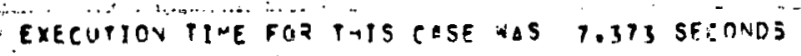

SAYP:_E PROBLEM 2 - RELEAST IN 3 PERIODS 


\section{DOSE RATE FACTORS}

STABILITY PASCURLL F RELEASE PERIDD

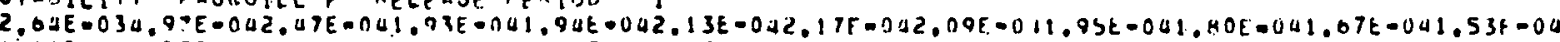

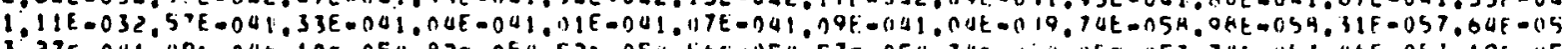

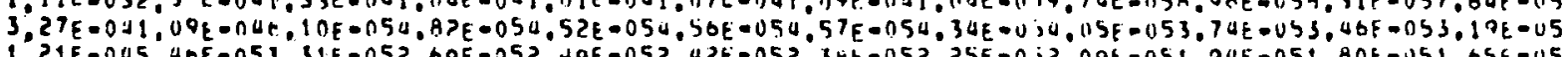

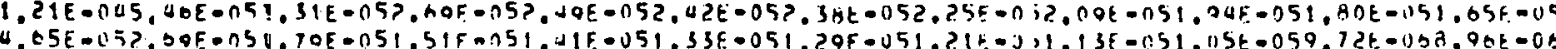

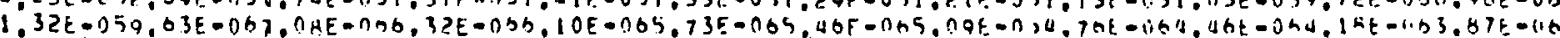

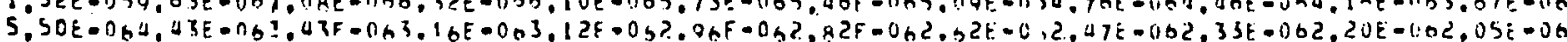

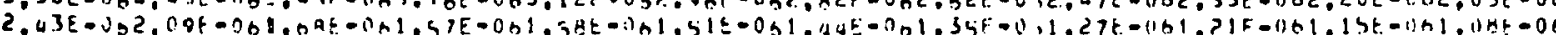

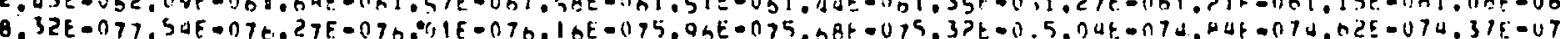

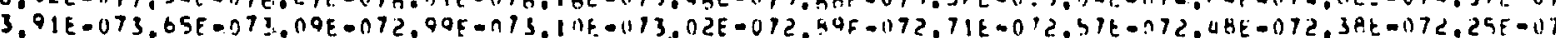
STABILITY PASDUILL F RELEASE PEGilio

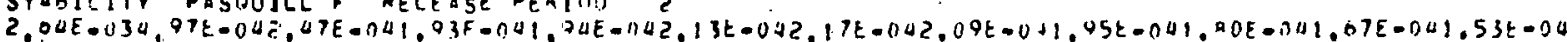

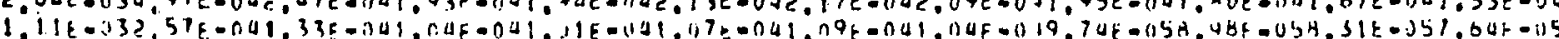

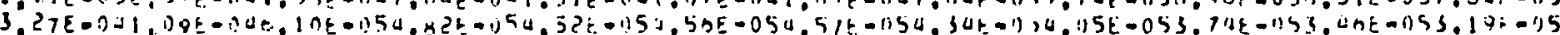

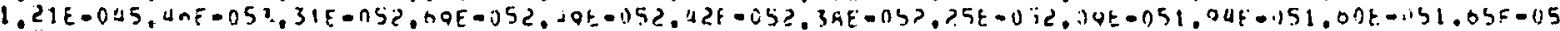

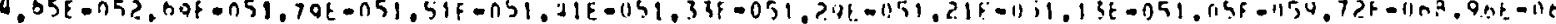

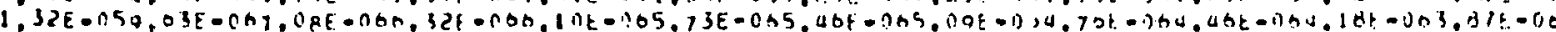

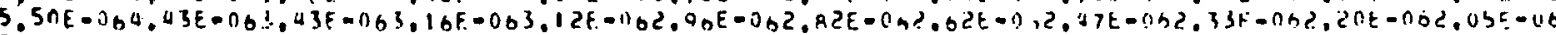

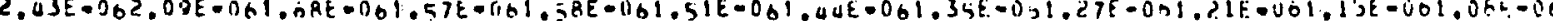

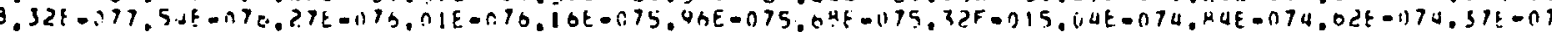

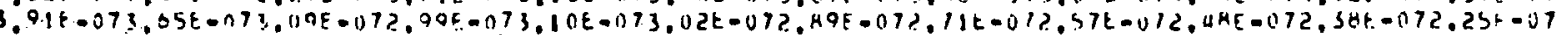
STA9ILITY CASLUTLL. D TELFASE DERTCO

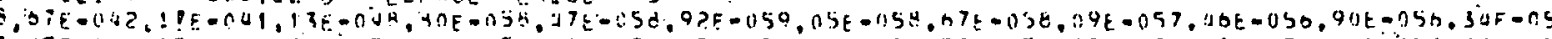

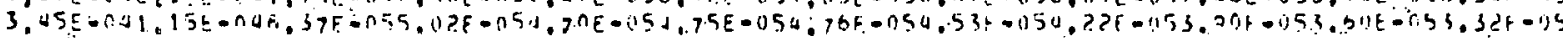

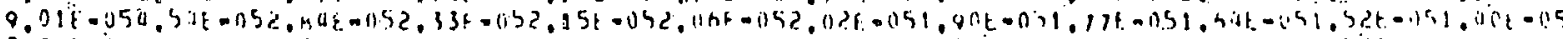

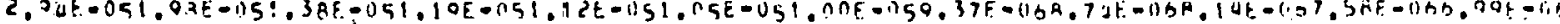

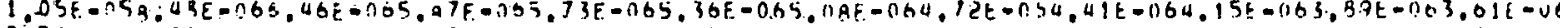

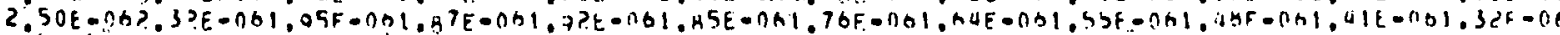

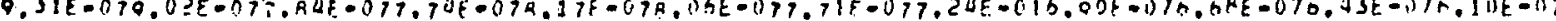

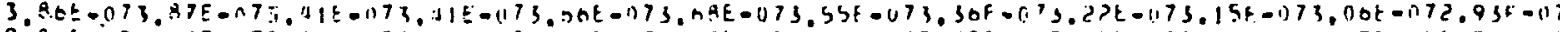

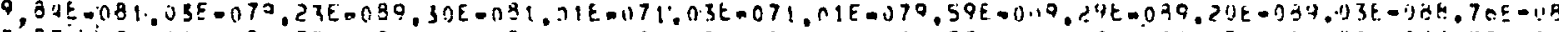

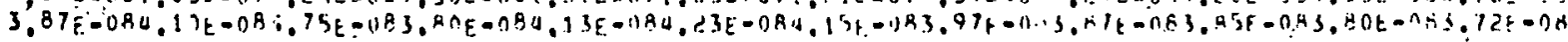




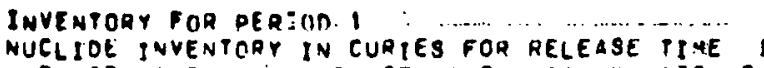

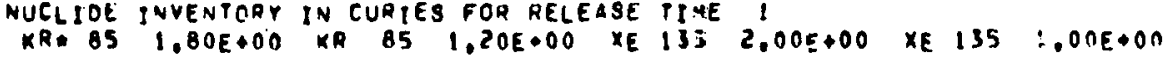

INVENTORY FOR DER:UDO 2
NUCLIOE INVENTORY IN CURIES FOR RELEASE TIME 2

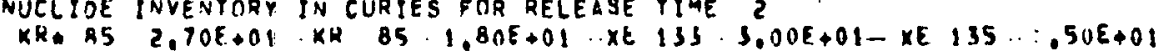

INYERTORY FOR DEFION 3

- $1,80 E+01 \cdots \times E 133 \cdot 3,00 E+01-\times E 135 \cdots: .50 E+01$

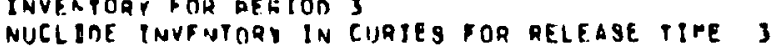

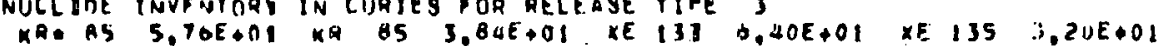

SIGMA $Y$ ANO 2 FOF SPARILIIY I

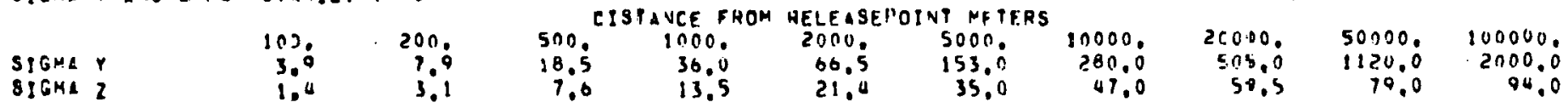

SIGMA Y ANU 2.EOR STABILI'Y -

SISTANCE fROM RELEASEPOINT METERS

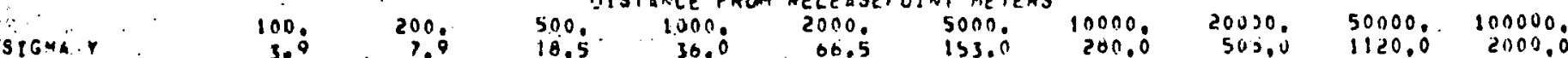

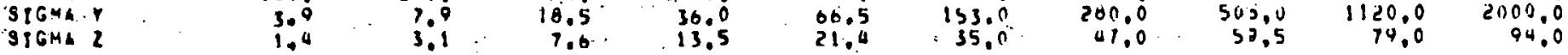

SIGMA Y ANO 2 FOR STAAILITY. 3

$\therefore$ TISTANCE FROH RELEASEPOTNT METERS

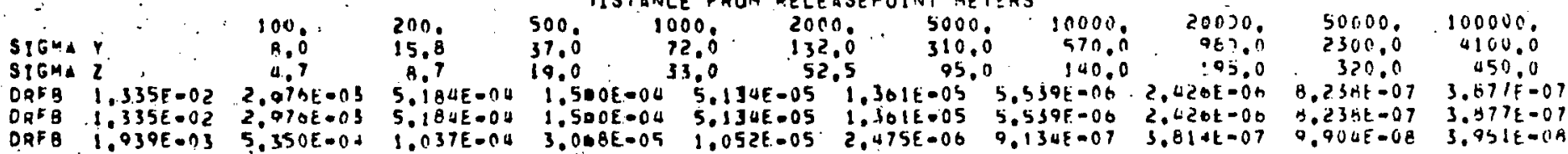


SaMPLE PROBLEM 2 - RELEASE IN 3 PEKIODS

DAIE $08 / 12175$

\section{DOSE TYPE}

GAMMA O, CM

GAMMA S. CM

BETA T, MGICM?

BETL 100, ME/EML

$S \times I N$

EYE

DOSE fOr 30,0 MIN. aelease periou, STABILITY pasquill F helease hejght o, meters

\begin{tabular}{|c|c|c|c|c|c|c|c|c|c|}
\hline 10. & $201 \%$ & & 1) & $\begin{array}{l}\because \text { METES } \\
\text { ronn. }\end{array}$ & $c$ & Se wh & 20000 & & \\
\hline & S. $8 B 5 E=05$ & sin & $55 ! t=05$ & $E=06$ & .06 & $1.4305=00$ & $(-1)$ & 01 & $t=0 \theta$ \\
\hline & $5.850 E=115$ & $2.514 t .05$ & & & & & 4 & $E=07$ & I 2 ram \\
\hline & $2.445 E-03$ & $4.2325=04$ & 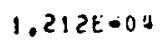 & $40-5-0=$ & & $745-00^{\circ}$ & $1.411 t=00$ & $.003 t=07$ & \\
\hline & & & & & -07 & $2.477 E-017$ & QHE - UB & $1.6455=08$ & \\
\hline & & - & & 每 & $E=0 S$ & PIIE- & $1.961 E=06$ & $E-07$ & $1.078 t$ \\
\hline & $u \cap \Delta \varepsilon=0 u$ & $914 F-05$ & $397 E=05$ & $10 B E-05$ & $3.907 E-86$ & $1.084 t=00$ & $0.562 E-07$ & $1.422 \varepsilon-07$ & $0.078 t=08$ \\
\hline
\end{tabular}

SXIN OOSE IS 0.0 CM GAMMA nOSE - T, MG/CML HETA DOSE.

EYE DOSE IS 0.0 CM GAMMA DOSE 100 . MG/CME BETA DOSE. 
DOSE IYPE

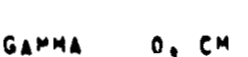
GAYMA DOSE PMIS TYPE

GAMMA 5. CM IOTAL OOSE THIS TYPE

BETA TO' MG/CIM?

TOPAL DOSE IHIS TYPE

BET. 100. MG/CM?

TOTAL OOSE TMIS TYPE

SxI4

TOTAL OOSE THIS IYEE

EYETLL OOSE THIS TYPE
DUSE FOH 1

100

$1.524 E-03 \quad 7.700 E=0$

0.
0.02
0.08

500 .

PFRIDE, STAGILITY DA
DISTANCE, MFTERS
IOOC.

2nno.

SORO.
SOLL $F$.

QFLEASE HEJGHT 0 , METERS

$3.333 E=04 \quad 1.752 E=64 \quad 0.331 E-05 \quad 3.642 E-05$

$1.884 E-03 \quad 6.540 E-n$ 2.01AE-04 $1.48 n E-64 \quad 7.93 A E-05 \quad 3.109 E-n 5$

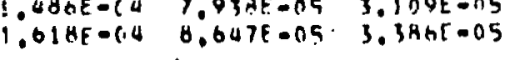

20000

50003.

100000 $1.6954-05 \quad 6.7109 k-116 \quad 1.591 E-06 \quad 4.968 E-0 !$

$1.272 E-01 \quad 2.954 E=02 \quad 5.1205-03$

$1.469 t-(.3 \quad 4.945 t-04 \quad 1.250 t=04$

1.40110 .05

$5.695 E=00 \quad 1.319 t-06$

$3.902 t \cdot c$ ?

3.3045 .09
3.3045 .09

$5,3.52 \varepsilon=04$

$.3525=04$

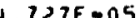

$0,127 F-05$
$5,135 t=n 5$

$1.820 t=05 \quad 4.8501=00$

$4.850 t=00$

$1.926 t=00$

$1.913 E=03$

$.343 E-01 \quad 3,032 E-0 ?$

$5.2535-03$

$1,03 \cap E=14$

$3.1006-n 2$

7.4081 .06
$9.4996=06$

$3,4+15-00 \quad 1.0145-00$

$2.154 E-1) \quad 0.115 t-05^{\circ}$

$2.0585=20$

1.4545 .01 3,2B3E-n2 $5,70 B E-03$

hautecs
$1,7 A 1 t-C_{3}$

S.870.t-D 4

$1.614 t .0104$

$3, U \div 6 f=00$

$1.0145=00$

$1.313 E-0 z \quad 2.090 k-03 \quad 6.641 E-04$

$2.69 H E=14 \quad 1.249 F=04$

$1.74 \times 6.14$

$0.3725 .05 \quad 2.407 t=115 \quad 0.448 t-00$

$0,9225=0.0$

$5.423 t=00$

2.0305-03 $\quad, 232 E-00 \quad 2,037 E=04 \quad 1,3605=04$

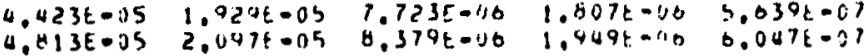

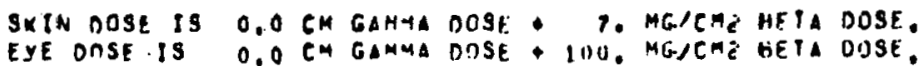

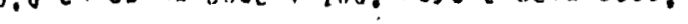




\section{DOSE TYDE.}

100.

200.

DISTANCE, METERS
DIOOD.

1000.

2000 .

5000,

ELFASE MEIGHT O, NEIEOS

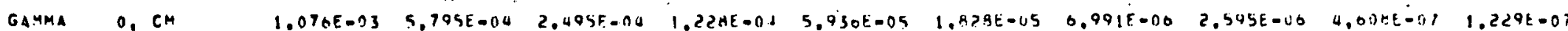

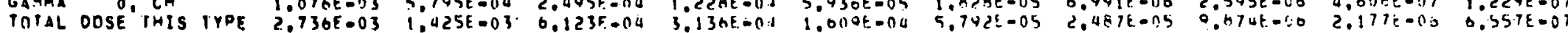

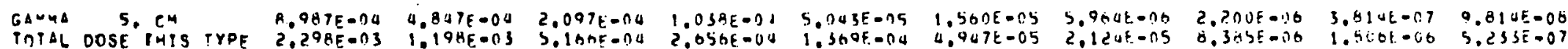

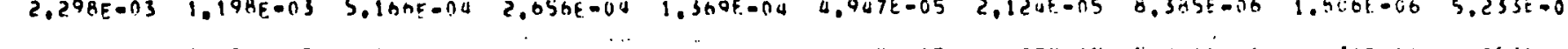

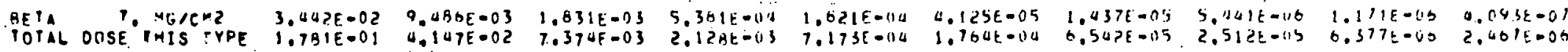

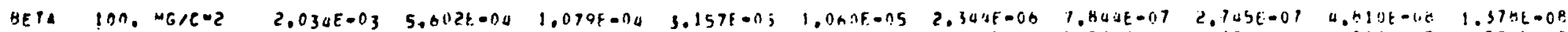

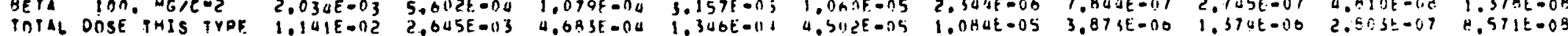

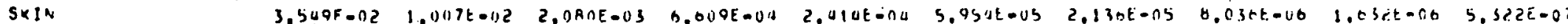

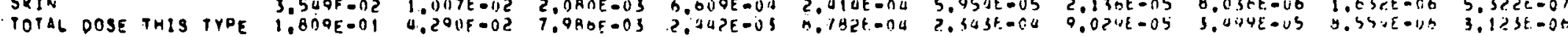

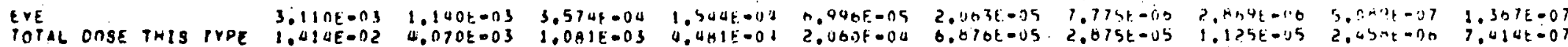

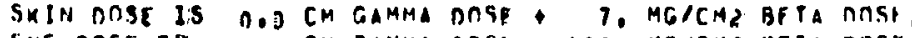


SAMPLE PROALEM 2 - PELEASF. IN 3 PERTDDS

DATE OU/12/75

NOKMALILEO ALR COHCENTRATION

PERIOO SPABIL:TY

DISTANCE, ME.TERS

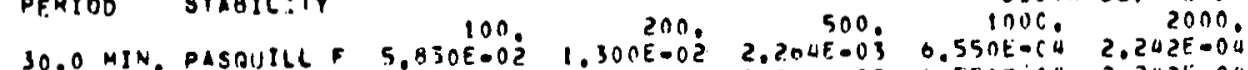

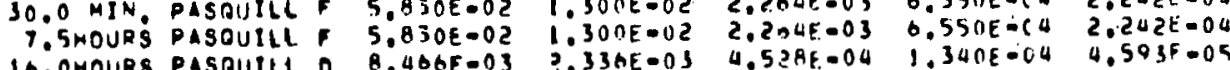

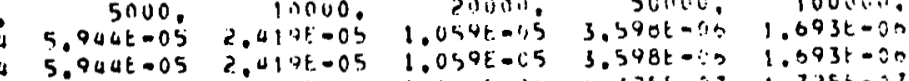

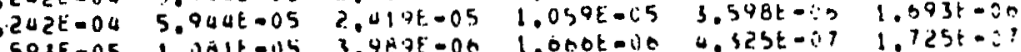
1.001t-15

EXECUTION IIME FOR THIS CASE WAS 2,884 SECONOS 
APPENDIX E

CODE FLOW DIAGRAMS 


\section{APPENDIX E}

\section{CODE FLOW DIAGRAMS}

This appendix gives general flow diagrams for program logic. Diagrams for BELI, BIVAR and SUBDOSA are given in Figures $E-1, E-2$, and $E-3$ respectively. 

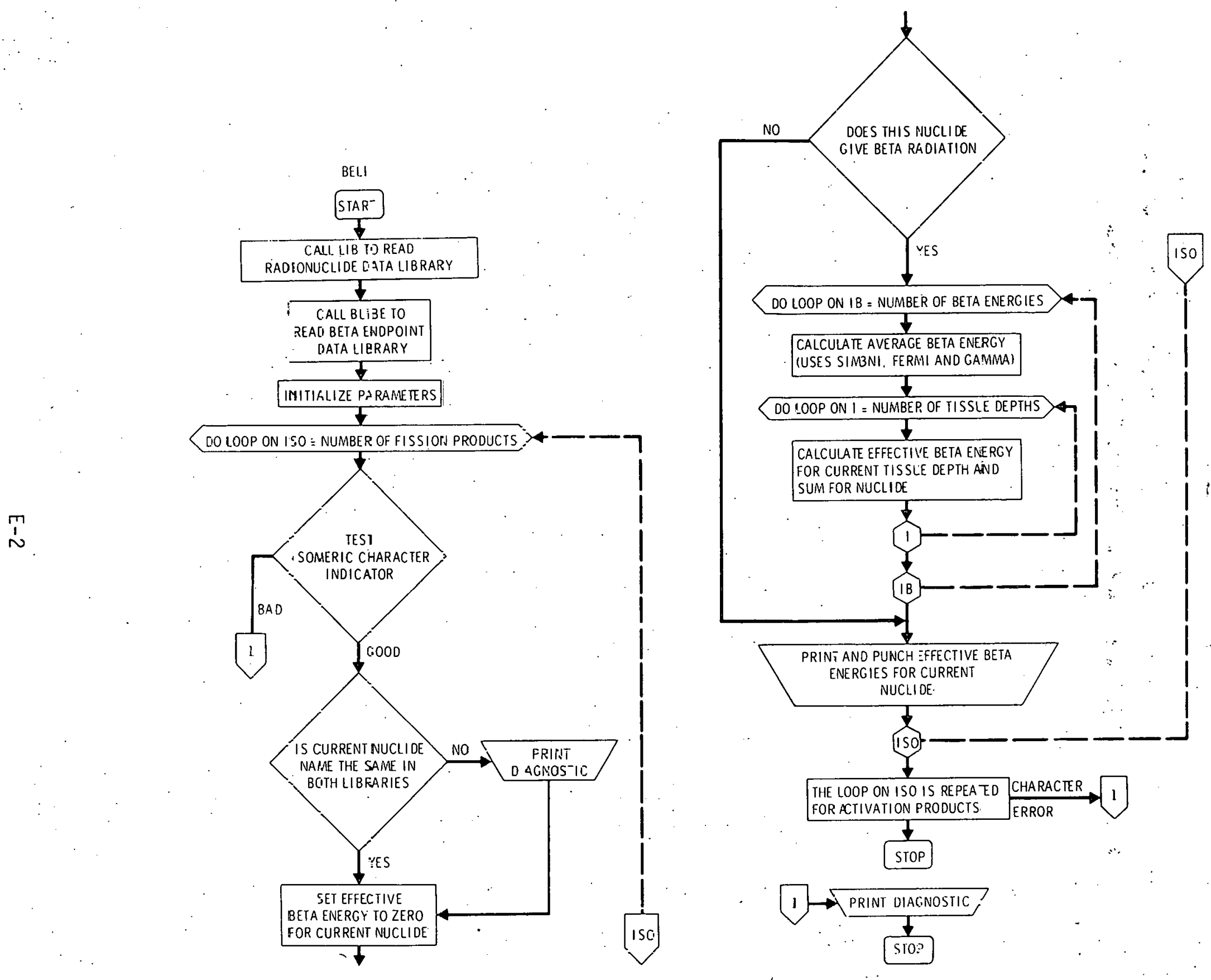

FIGURE E-1. Flow Diágram for BELI 


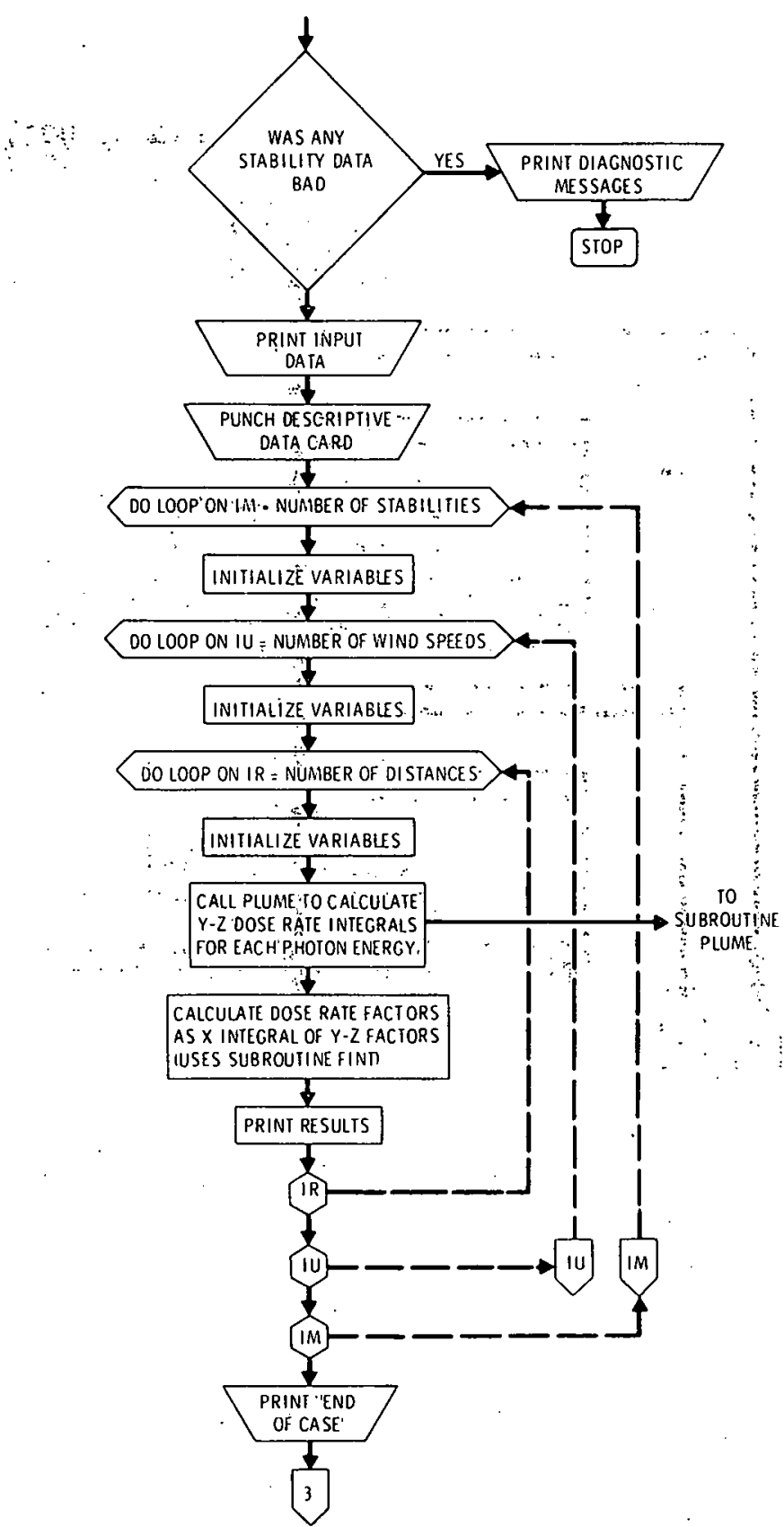

FIGURE E-2. Flow Diagram for BIVAR 

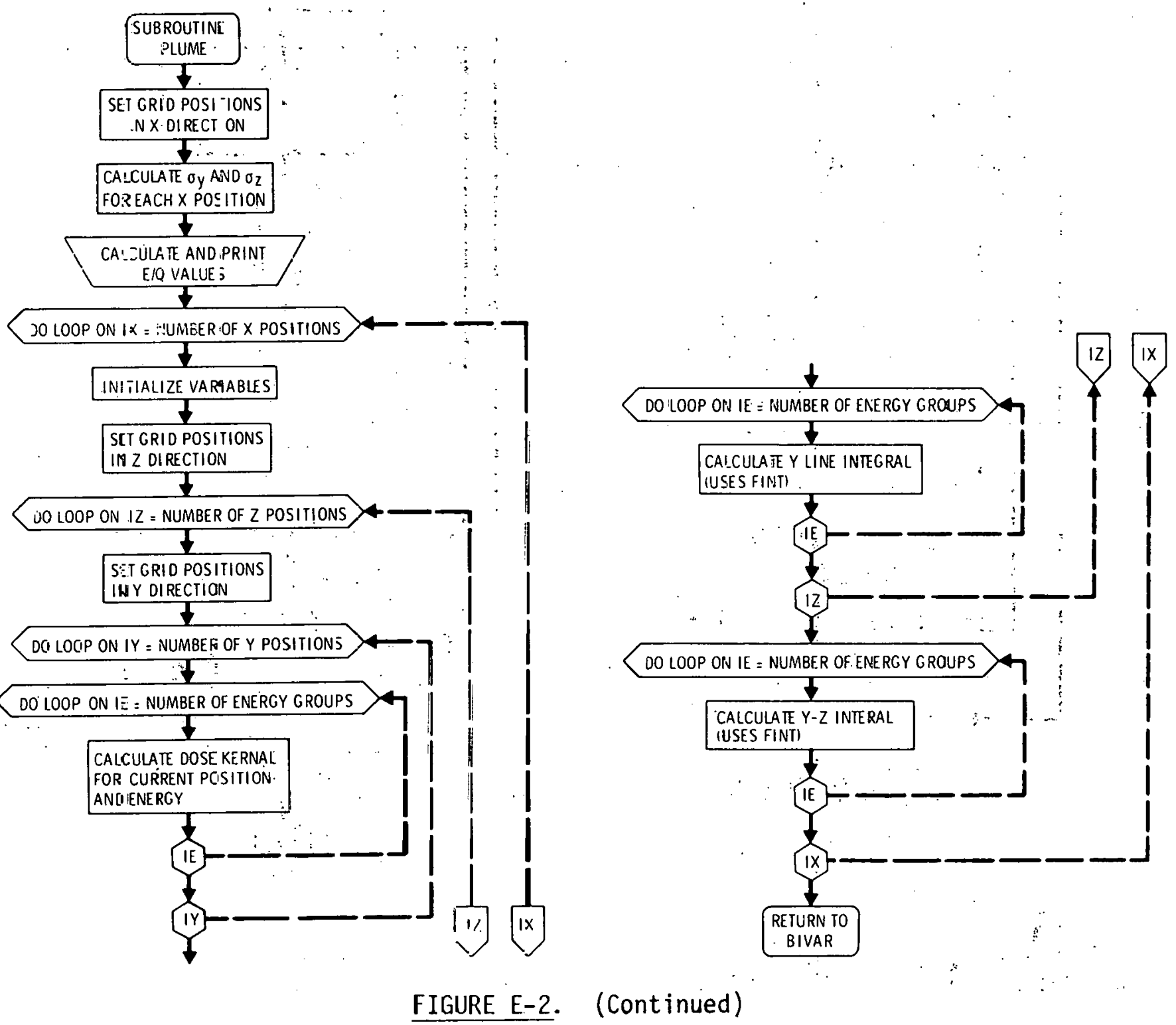

FIGURE E-2. (Continued) 


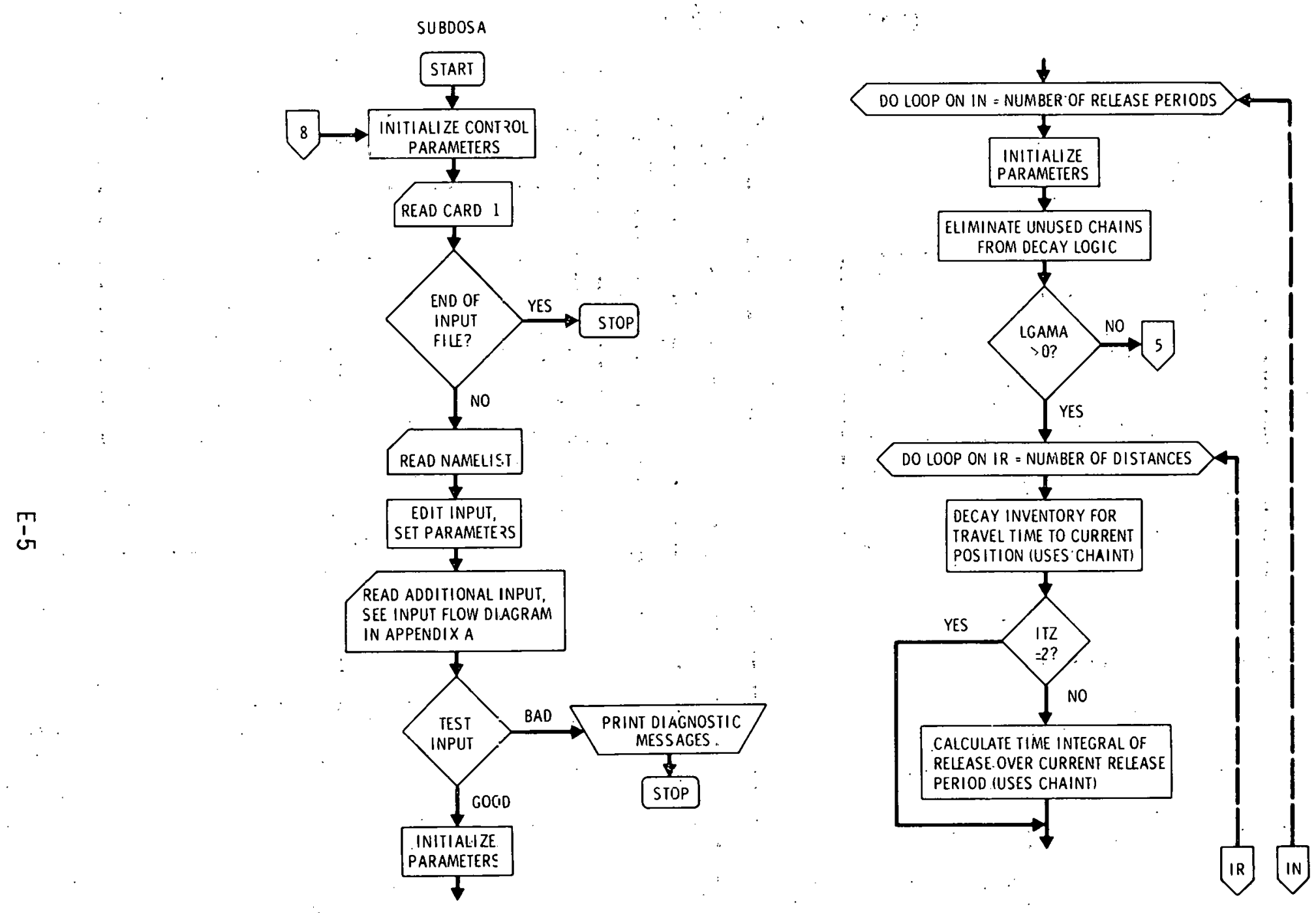

FIGURE E-3. Flow Diagram for SUBDOSA 


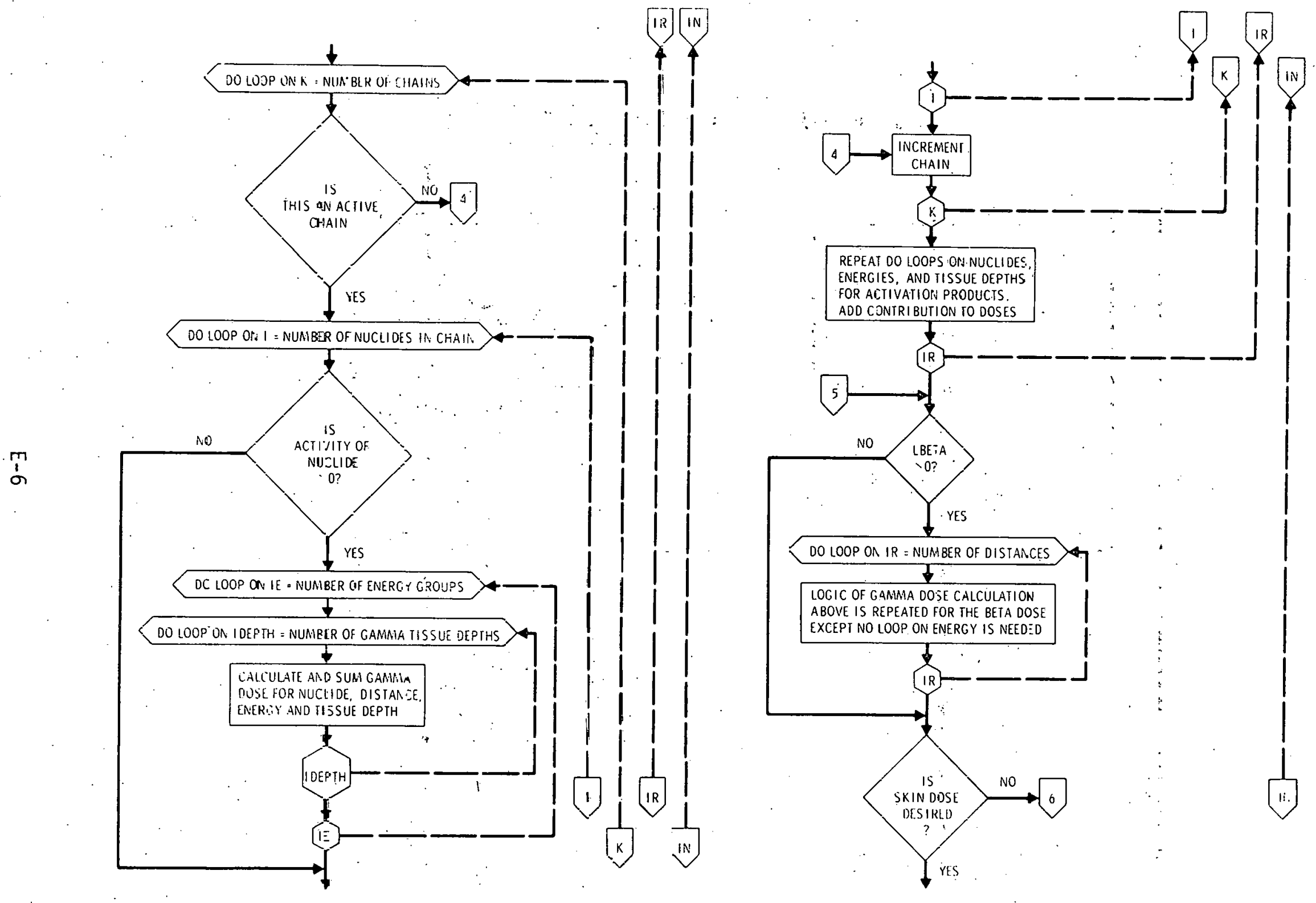

FIG'JRE E-3. (Continsed) 


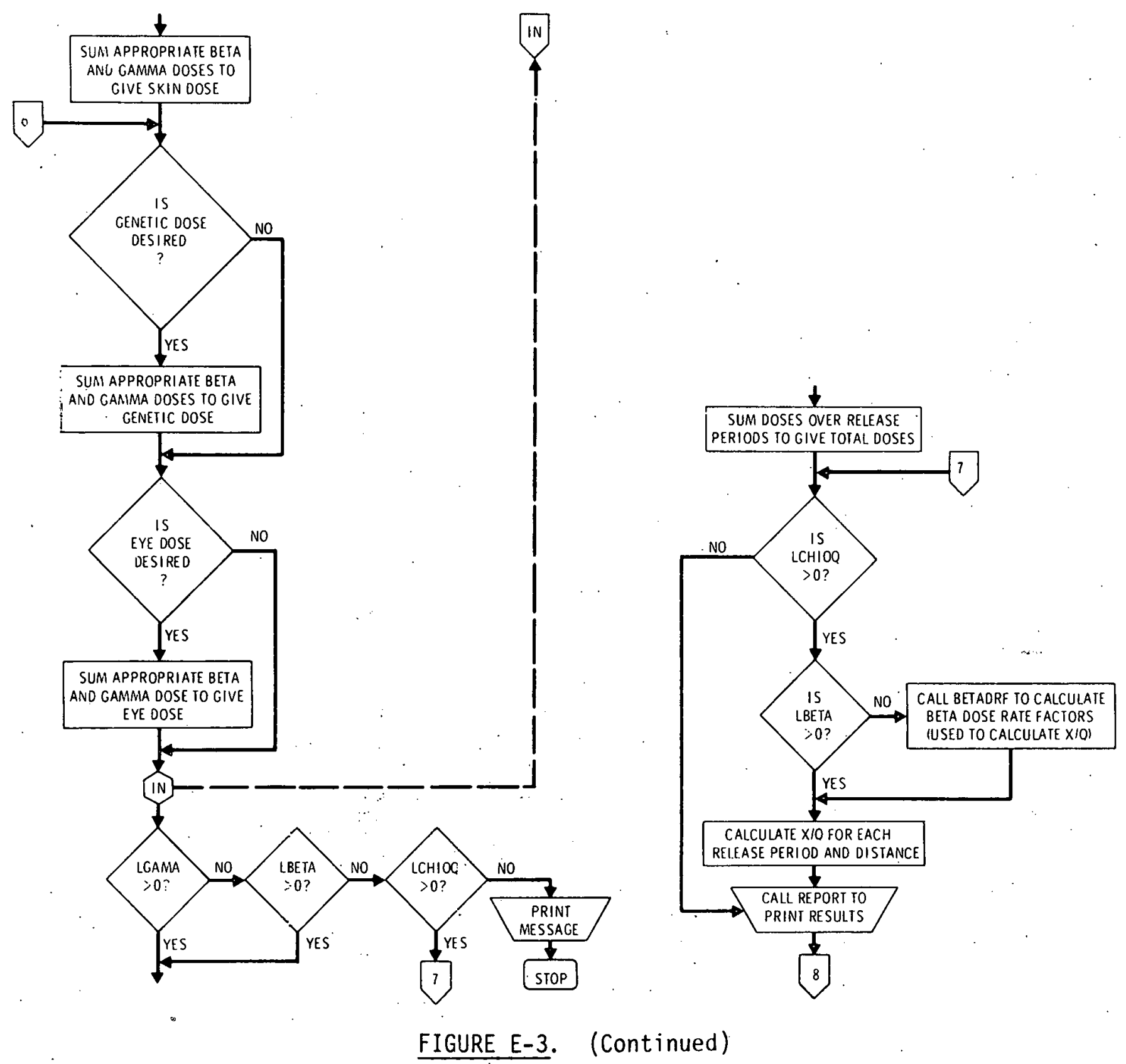




\section{DISTRIBUTION}

No. of

Copies

OFFSITE

209
No. of

Copies

OFFSITE

F. T. Binford

Hol ifield National Laboratory

P.0. Box $X$

Oak Ridge, TN 37830

K. E. Cowser

Hol ifield National Laboratory

P.0. Box $X$

Oak Ridge, TN 37830

ERDA Health Services Laboratory (Idaho Falls)

Idaho Falls, ID 83401

R. E. Baker

NRC Office of Regulation

Nuclear Regulatory Commission

Washington, DC 20555

C. C. Gamertsfelder

NRC Office of Regulation

Nuclear Regulatory Commission

Washington, DC 20555

E. H. Markee

NRC Office of Regulation

Nuclear Regulatory Commission

Washington, DC 20555

P. 0. Strom

NRC Office of Regulation

Nuclear Regulatory Commission

Washington, DC 20555

R. L. Waterfield

NRC Office of Regulation

Nuclear Regulatory Commission

Washington, DC 20555

F. Swanberg

NRC Office of Regulation

Nuclear Regulatory Commission

Washington, DC 20555
2. B. F. Maskewi tz Radiation Shielding Information Center P.0. Box X

Oak Ridge, TN 37830

I. Van der Hoven

Chief, Air Resources Environmenta? Laboratory

National Oceanic and Atmospheric

Administration

Room 1625B

8060 13th St.

Silver Spring, MD 20910

196 ERDA Technical Information

Center

ONSITE

1 ERDA Richland Operations Office

B. J. Melton

1 Atlantic Richfield Hanford Company

G. E. Backman

2 Hanford Engineering Development Laboratory

B. W. Bentley

R. 0. Budd 
No. of

Copies

1. United Nuclear Industries

C. D. Corbit

1 Washington Public Power Supply System

F. E. Owen

75 Battellc-Northwest

D. A. Baker

C. E. Elderkin

J. P. Corley

J. G. Droppo

T. W. Horst

J. R. Houston

H. V. Larson

L. C. Schwendiman

J. K. Soldat

D. L. Strenge (40)

E. C. Watson (10)

Technical Information Files

Technical. Publications

Distr-2 